\title{
Amino Acid Biosynthesis and the COP9 Signalosome in Aspergillus nidulans:
}

Regulatory Networks in a Filamentous Fungus

\author{
Dissertation \\ zur Erlangung des Doktorgrades \\ der Mathematisch-Naturwissenschaftlichen Fakultäten \\ der Georg-August-Universität zu Göttingen
}

\author{
vorgelegt von \\ Silke Busch \\ aus Vechta
}

Göttingen 2002 
Die vorliegende Arbeit wurde in der Arbeitsgruppe von Prof. Dr. Gerhard H. Braus in der Abteilung Molekulare Mikrobiologie des Institutes für Mikrobiologie und Genetik der Georg-August-Universität Göttingen angefertigt.

Teile dieser Arbeit wurden veröffentlicht in:

Busch, S., Hoffmann, B., Valerius, O., Starke, K., Düvel, K. and G.H. Braus. 2001. Regulation of the Aspergillus nidulans hisB gene by histidine starvation. Curr Genet 38: 314-322.

Busch, S., Bode, H.B., Brakhage, A.A. and G.H. Braus. 2002. Impact of the crosspathway control on regulation of lysine and penicillin biosynthesis in Aspergillus nidulans. Accepted for publication (08 / 2002) in Curr Genet.

D 7

Referent: Prof. Dr. G.H. Braus

Korreferent: Prof. Dr. G. Gottschalk

Tag der mündlichen Prüfung: 31.10.2002 
Für meine Eltern 



\section{Contents}

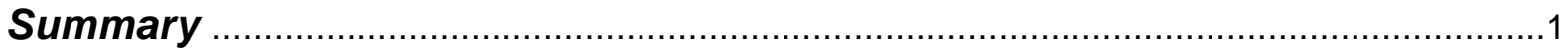

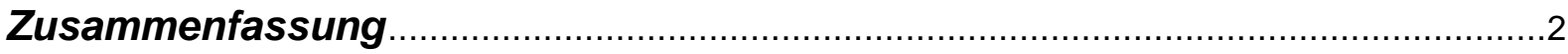

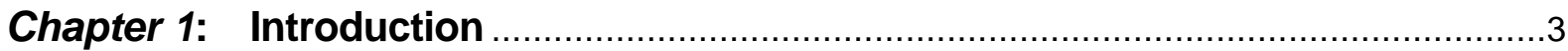

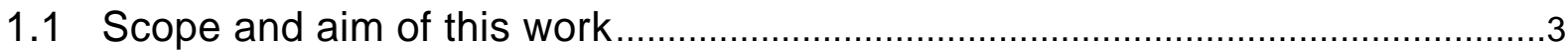

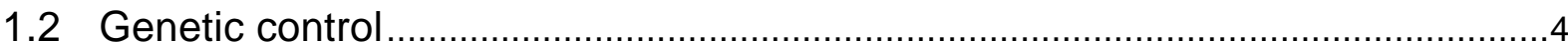

1.2.1 Expression of gene products and the cross-pathway control ............................4

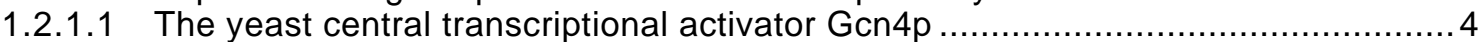

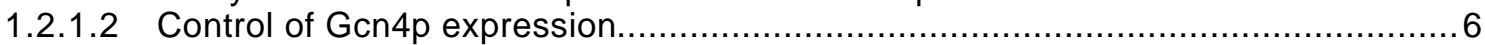

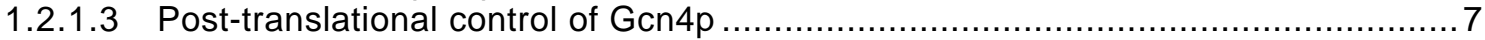

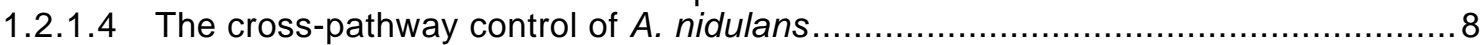

1.2.2 Degradation of gene products and the COP9 signalosome.................................

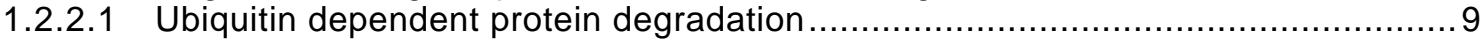

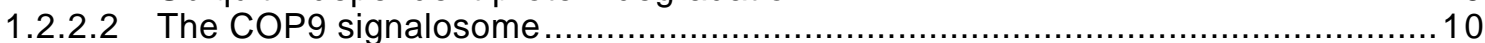

1.2.2.3 Physiological role of the COP9 signalosome .................................................... 13

1.2.2.4 The COP9 signalosome in lower eukaryotes. ............................................ 14

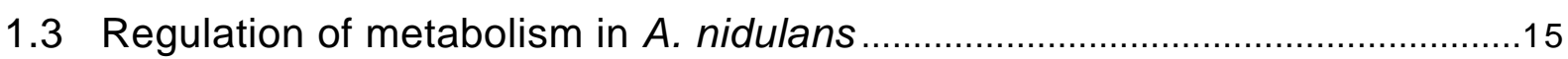

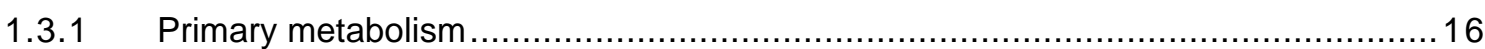

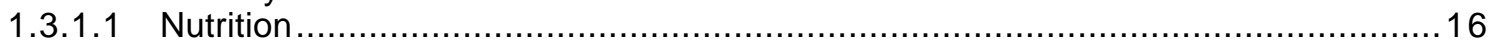

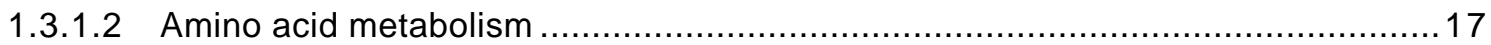

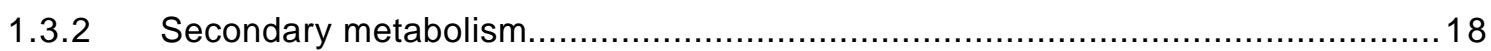

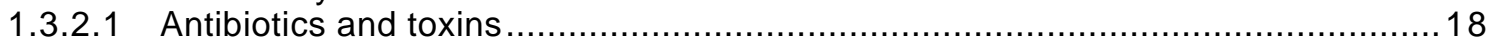

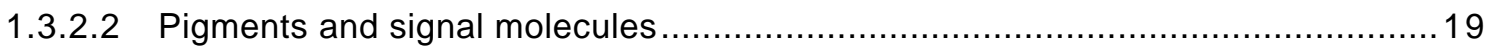

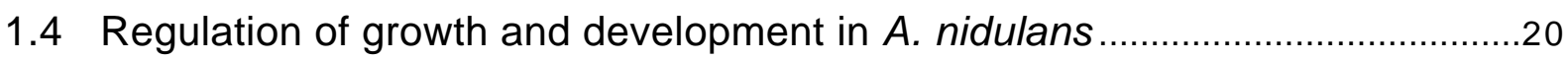

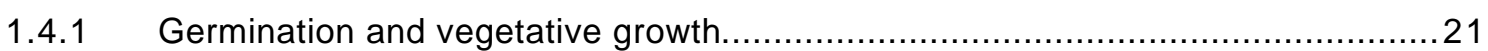

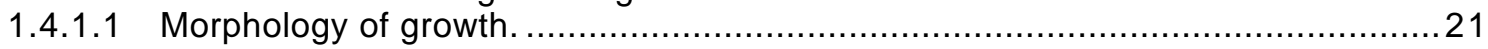

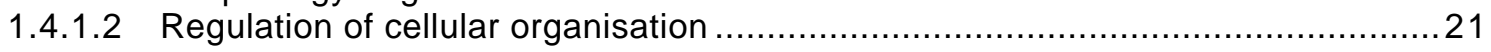

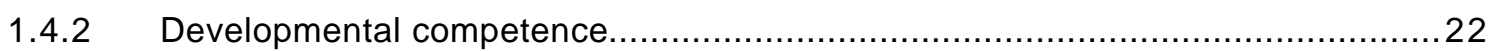

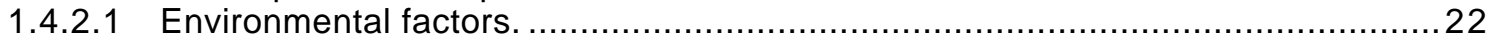

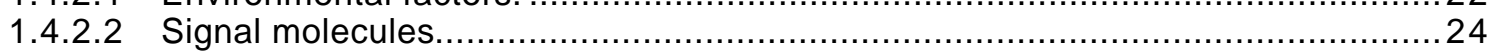

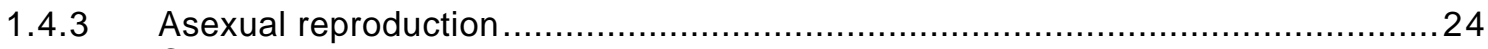

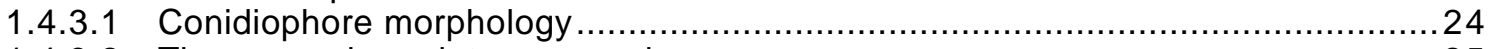

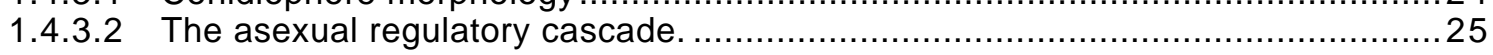

1.4.4 Sexual reproduction and developmental co-ordination ...................................25

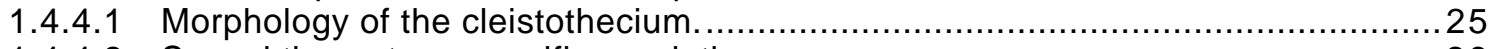

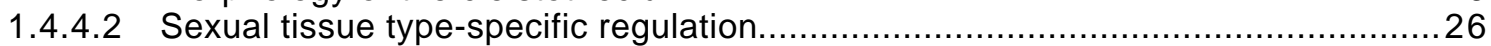

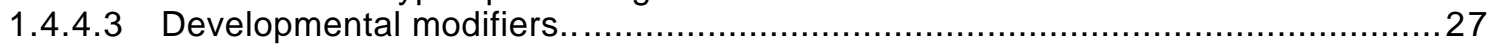

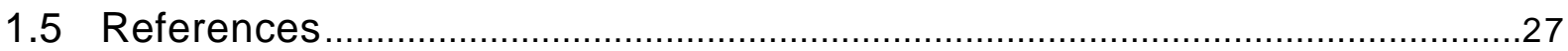




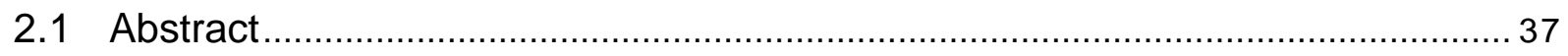

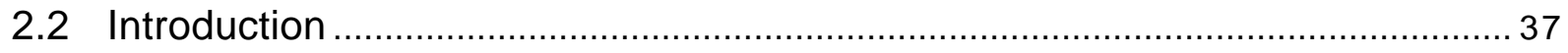

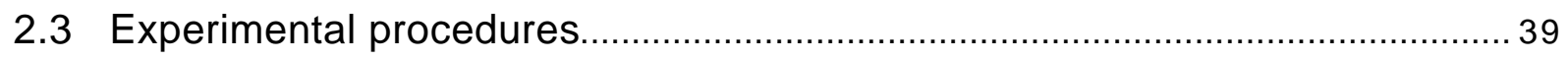

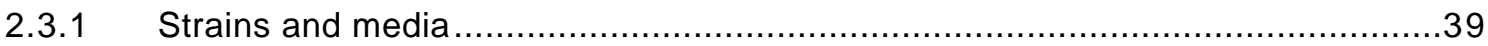

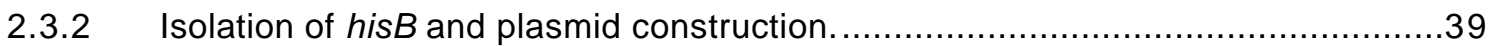

2.3.3 Recombinant DNA techniques and computational analyses............................. 40

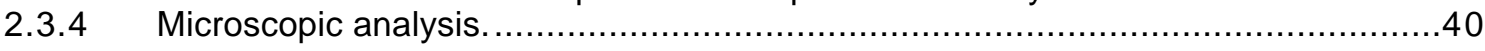

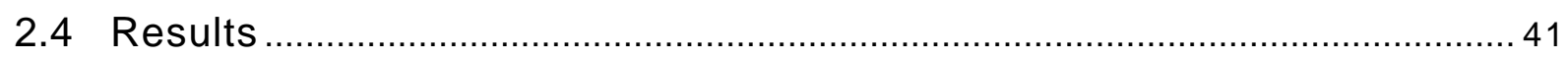

2.4.1 The hisB gene of Aspergillus nidulans encodes the imidazole glycerol-phosphate dehydratase of the histidine biosynthetic pathway.................41

2.4.2 Construction of an $A$. nidulans hisB deletion strain auxotroph for histidine............41

2.4.3 The his $B$ gene of $A$. nidulans is regulated on the transcriptional level under amino acid starvation conditions...............................43

2.4.4 Growth on low histidine concentration causes
induction of the cross-pathway control in the his $B$ deletion mutant......................45

2.4.5 Deletion of $h i s B$ affects sexual development of $A$. nidulans. .............................45

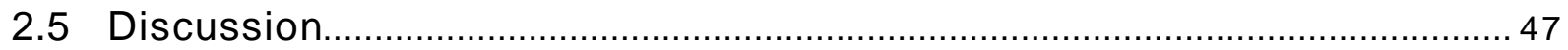

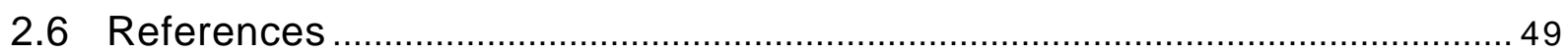

Chapter 3: Impact of the cross-pathway control on regulation of lysine and penicillin biosynthesis in Aspergillus nidulans.

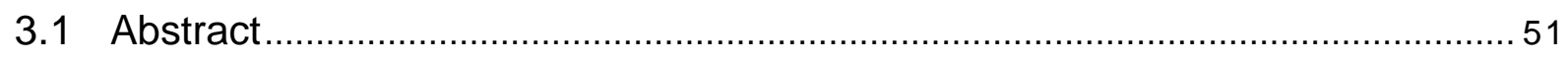

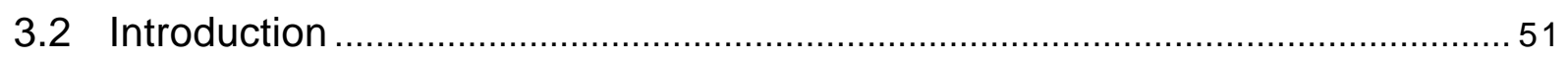

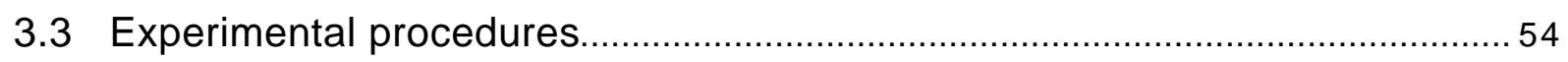

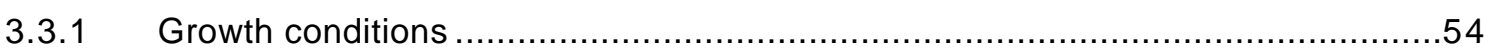

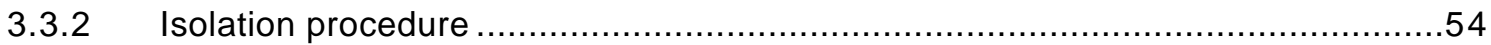

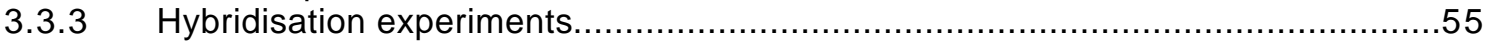

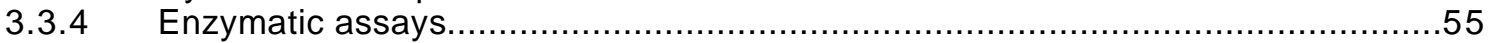

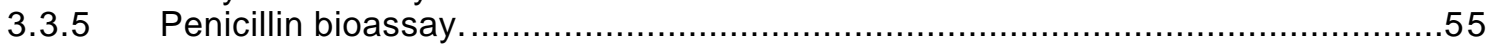

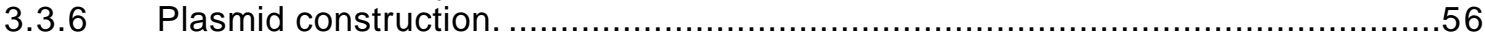

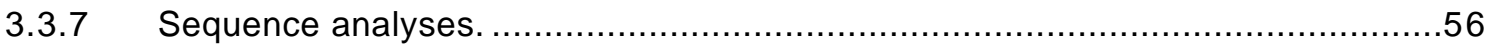

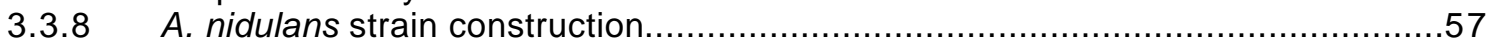

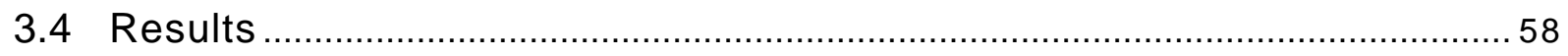

3.4.1 The IysA gene of $A$. nidulans shows up to $62 \%$ amino acid indentities to lysine-forming saccharopine dehydrogenases..............................58

3.4.2 Transcript levels are increased for lys $A$ but reduced for lys $F$ by starvation-dependent activation of CPCA .......................58

3.4.3 Steady state levels of lys $F$, Iys $A$ and $c p c A$ mRNA are reduced under penicillin production conditions..........................................60

3.4.4. CPCA overproduction decreases expression of the penicillin specific genes as well as the penicillin titre................................62

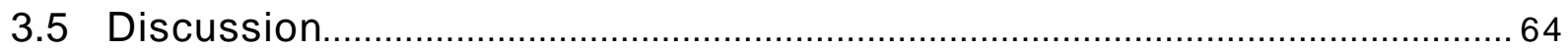

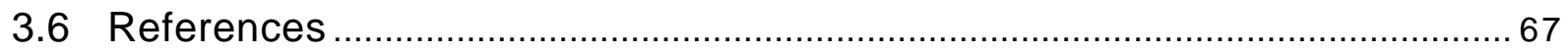




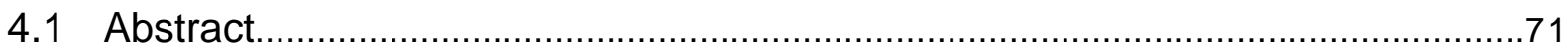

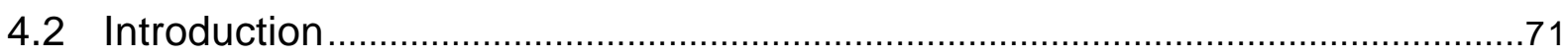

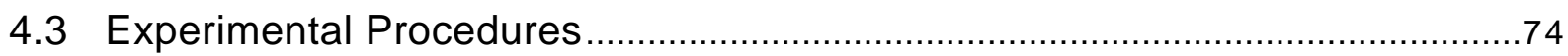

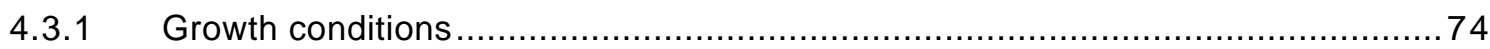

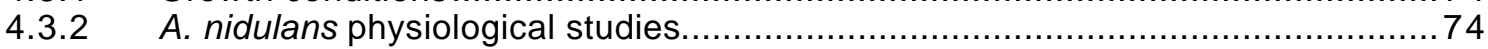

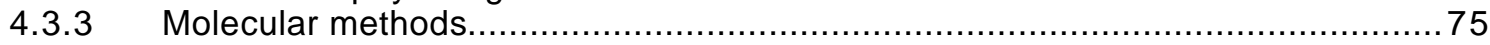

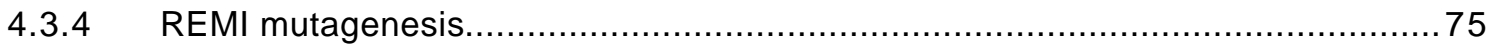

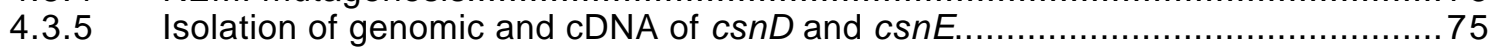

4.3.6 Construction of plasmids for A. nidulans manipulation .................................... 76

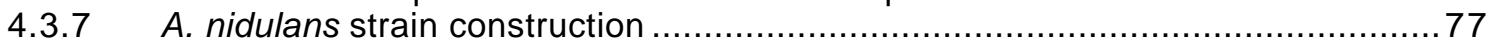

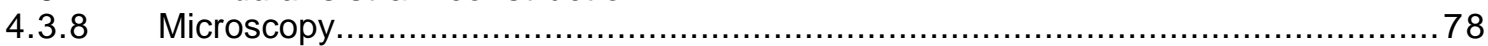

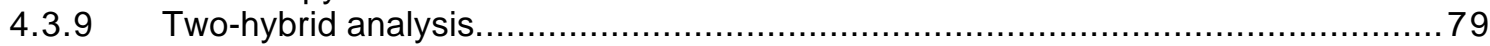

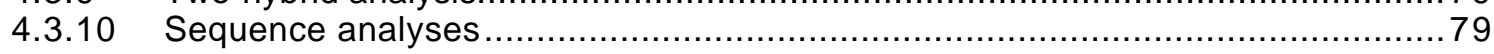

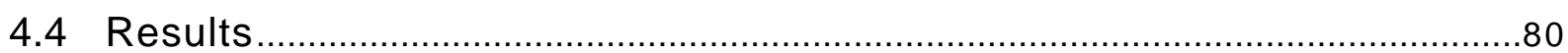

4.4.1 The A. nidulans csnD gene encodes a $\mathrm{PCl}$ domain protein similar to the fourth subunit of the COP9 signalosome ............................8 80

4.4.2 The $\operatorname{csn} D$ gene is constitutively transcribed through the entire life cycle of $A$. nidulans and its gene product is enriched in the nucleus ..............82 82

4.4.3 Deletion of the $\operatorname{csn} D$ gene blocks sexual development and causes altered pigmentation and cell morphologies in $A$. nidulans................83

4.4.4 An A. nidulans strain lacking the fifth CSN subunit (CSNE) shows a $\Delta c s n D$-like phenotype ............................... 84

4.4.5 The csnE and $\operatorname{csn} D$ gene products

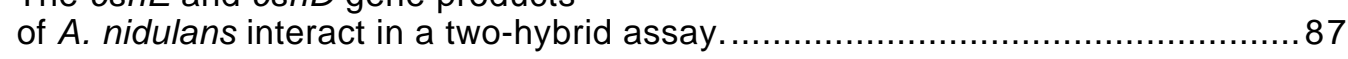

4.4.6 Light-dependence of development is disturbed in the $\operatorname{csn} D$ deletion strain

4.4.7 The velvet gene product does not release

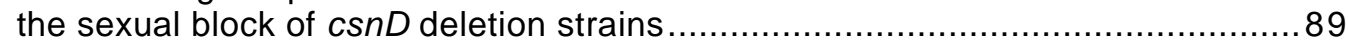

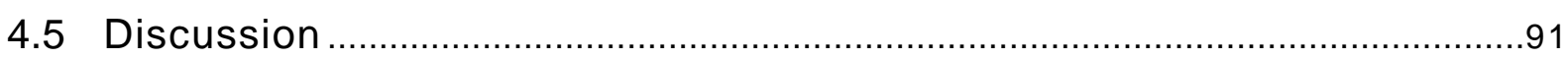

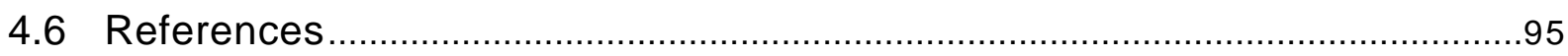

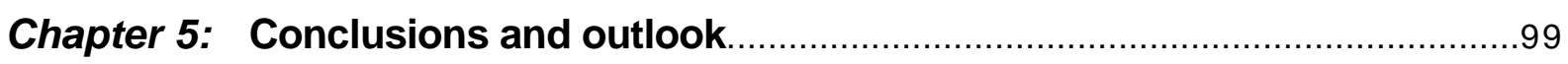

5.1 Amino acid biosynthesis and the COP9 signalosome in A. nidulans..............99

5.1.1 Signalling of the cross-pathway control .......................................................100

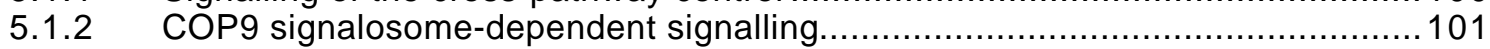

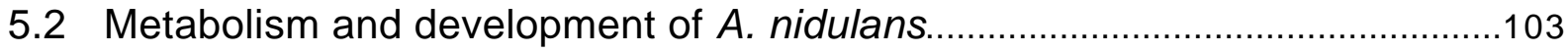

5.2.1 Morphogenesis of sexual fruit bodies........................................................... 103

5.2.2 Cross-connections of secondary metabolism and sexual development................. 105

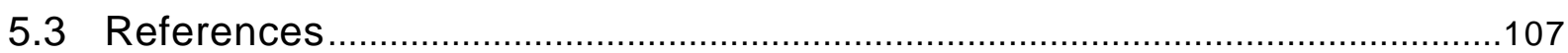





\section{Summary}

The filamentous fungus Aspergillus nidulans represents an eukaryotic model system versatile in metabolism and developmental properties. This work focuses on the impact of modified enzyme activities whithin two regulatory networks on the fungal organism. The cross-pathway control ensures proper biosynthesis of amino acids upon corresponding starvation conditions in fungi. The COP9 signalosome is part of a regulatory network which is essential for the development of higher eukaryotes. Components of both systems have been isolated from A.nidulans and their contribution to the molecular control of physiology and morphology was examined.

Two amino acid biosynthesis genes, his $B$ and $l y s A$, have been identified as target genes of the cross-pathway control. HISB, the imidazole glycerol-phosphate dehydratase (E.C. 4.2.1.19), is essential for histidine biosynthesis. Supplementation of a hisB deletion strain with traces of histidine enables growth of this auxotrophic strain. In contrast to the wild-type grown under the same conditions, this strain exhibits an induced cross-pathway control and arrests sexual development at the level of micro-cleistothecia. When high histidine supply represses the cross-pathway control, the sexual cycle can be complete. This indicates a link between the regulatory network of amino acid biosynthesis and sexual development in A. nidulans.

The lys $A$ gene, encoding saccharopine dehydratase (E.C. 1.5.1.7), catalyses the ultimate step of lysine formation in the branched lysine/penicillin biosynthesis pathway. Transcription of IysA is increased upon amino acid starvation due to an activated cross-pathway control, whereas the lys $F$ gene of the pathway's common stem as well as penicillin production were negatively affected. Thus, the regulatory network of amino acid biosynthesis is involved in a cross-talk between regulation of primary and secondary metabolism in A.nidulans.

The COP9 signalosome is a multiprotein complex with at least two assumed associated enzyme activities, a protein kinase and a deneddylase, which contribute to the regulation of targeted protein degradation. For the first time, two genes encoding subunits four and five of the COP9 signalosome of filamentous fungi, $\operatorname{csn} D$ and $\operatorname{csn} E$ of $A$. nidulans, were identified. Deletion of either csn subunit resulted in multiple mutant phenotypes. It can be concluded that the COP9 signalosome of A.nidulans is involved in repression of pigment production and maintenance of cell polarity in vegetative hyphae as well as in light-dependence of developmental induction and completion of the sexual cycle.

These results indicate that changes in single enzyme activities whithin genetic networks ultimately affect the metabolic and developmental potential of the entire fungal organism and suggest cross-connections between the different regulatory circuits. 


\section{Zusammenfassung}

Der filamentöse Pilz Aspergillus nidulans ist ein eukaryontischer Modellorganismus mit vielseitigem Metabolismus und Entwicklungspotential. Diese Arbeit befasst sich mit der Auswirkung von veränderten Enzymaktivitäten innerhalb zweier regulatorischer Netzwerke auf den pilzlichen Organismus. Das System der 'Allgemeinen Kontrolle' sichert in Pilzen die Biosynthese von Aminosäuren unter entsprechenden Mangelbedingungen. Das COP9 Signalosom ist Teil eines regulatorischen Netzwerkes welches essentiell für Entwicklungsvorgänge in höheren Eukaryonten ist. Komponenten aus beiden Systemen wurden aus A.nidulans isoliert und inr Einfluss auf physiologische und metabolische Prozesse untersucht.

Die beiden Aminosäure-Biosynthesegene hisB und IysA wurden als Zielgene der Allgemeinen Kontrolle identifiziert. Die Imidazol Glycerol-phophat Dehydratase HISB (E.C. 4.2.1.19) ist essentiell für die Histidin Biosynthese. Ein hisB-Deletionsstamm wächst wenn geringe Histidinmengen supplementiert werden, anders als beim Wildtyp zeigt er unter diesen Bedingungen jedoch eine aktivierte Allgemeine Kontrolle und arretiert seine sexuelle Entwicklung auf der Stufe der Mikro-Cleistothecien. Durch hohe Mengen an Histidin kann das Kontrollsystem abgeschaltet und die Entwicklungsblockade aufgehoben werden. Dies weist auf eine Verbindung zwischen dem regulatorischen Netzwerk der Aminosäure-Biosynthese und der sexuellen Fruchtkörper-Bildung von A.nidulans hin.

Das lysA-Gen kodiert für die Saccharopine Dehydratase (E.C. 1.5.1.7), welche den letzten Schritt der Lysin-Bildung des verzweigten Lysin/PenicillinBiosyntheseweges katalysiert. Die Transkription von lysA wird bei AminosäureMangel durch die Allgemeine Kontrolle erhöht, wohingegen die Transkription des lysF-Gens, welches im gemeinsamen Teil des Syntheseweges agiert, sowie die Penicillin Produktion negativ beeinflusst werden. Das regulatorischen Netzwerk der Aminosäure-Biosynthese ist demnach an einer Co-Regulation zwischen Primär und Sekundärmetabolismus in A.nidulans beteiligt.

Das COP9 Signalosom ist ein Multiprotein-Komplex mit mindestens zwei assoziierten Enzymaktivitäten, eine Proteinkinase und eine Deneddylase, welche zur Regulation gezielter Proteindegradation beitragen. Mit $\operatorname{csn} D$ und $\operatorname{csn} E$ konnten Gene der CSN-Untereinheiten vier und fünf erstmals aus einem filamentösen Pilz isoliert werden. Deletion von $\operatorname{csn} D$ oder $\operatorname{csnE}$ führte zu multiplen mutanten Phänotypen. Diese lassen darauf schliessen, dass das COP9 Signalosom in A. nidulans an der Repression von Pigmentbildung und dem Erhalt von Zellpolariät in vegetativen Zellen sowie an der Lichtabhängigkeit der Reproduktionszyklen und dem Abschluss der sexuellen Entwicklung beteiligt ist.

Die Ergebnisse zeigen, dass Veränderungen in einzelnen Enzymaktivitäten innerhalb komplexer genetischer Netzwerke Auswirkungen auf Metabolismus und Entwicklungvorgängen des pilzlichen Organismus haben, und lassen vermuten, dass die einzelnen Regulationskreise miteinander verbunden sind. 


\section{Chapter 1}

\section{Introduction}

\subsection{Scope and aim of this work}

Resolution of the DNA structure about 50 years ago (Watson and Crick 1974) gave rise to a new scientific field, molecular genetics, that proceeded vastly to our current knowledge of the molecular design of life. What started with the identification of single genes has now come to a point of complete genome sequencing. It is no more primarily the discovery of distinct biochemical pathways that inspires the research community but broader studies on overall cellular organisation driven by new technologies like 'transcriptomics' and 'proteomics'. Subjects of special interest are regulatory networks that control molecular processes in the living organism in its entirety. The understanding of such sophisticated genetic regulations is indispensable for applied molecular biology, especially for industrial metabolic engineering that aims to modulate the metabolic flux to a desired biomolecule.

Several economically, medically and agriculturally important fungal species belong to the genus Aspergillus. For example, organic acids like citric acid and extracellular enzymes like glucoamylases are produced at industrial scale by $A$. niger and $A$. awamori, respectively. Bioactive molecules like $B$-lactam antibiotics and aflatoxins are excreted by $A$. fumigatus and $A$. flavus. Additionally, A. fumigatus is an opportunistic pathogen causing invasive aspergillosis in immuno compromised individuals (Kontoyiannis and Bodey 2002). A. nidulans constitutes a representative of this fungal genus that is capable of complex biosyntheses and differentiation processes. This eukaryotic model organism is accessible to molecular methods and can be manipulated relatively easy. Thus, Aspergillus is particularly suited for studying regulatory networks of the molecular crossconnections between environmental stimuli, metabolism and development.

This study directs the focus on enzyme activities involved in two regulatory networks: the cross-pathway control of amino acid biosynthesis and the COP9 signalosome-dependent system cotrolling development. Components of both networks of $A$. nidulans were isolated and analysed with a special focus on their impact on metabolism and development. Two target genes of the cross-pathway control were examined, which revealed an impact of this network in sexual reproduction (Chapter 2) and penicillin biosynthesis (Chapter 3). Additionally, two subunits of the $A$. nidulans COP9 signalosome were shown to be essential for light-dependent signalling, pigment production and development (Chapter 4). Thus, the cross-pathway control and the COP9 signalosome-dependent network are involved in the regulation of environmental stimuli, physiology and morphology. 


\subsection{Genetic control}

A major maxim of the living cell is to code information within the order of base residues of nucleic acids and selectively transfer this information as ribonucleic acids to finally produce the corresponding proteins as e.g. biocatalysts of cellular processes. This implies that different molecular levels, including DNA transcription, RNA translation and protein degradation, are point of regulatory attack (Fig. 1.1). Common regulatory themes on each level are direct modification, changes in conformation and stabilisation. For the compartmentalised eukaryotic cell and multicellular organisms, localisation and intercellular communication define an additional level of regulation. For molecular control of physiological and morphological processes, several of these regulatory principles are combined to perceive extracellular and intracellular signals and finally respond with an adequate cellular answer. Two eukaryotic regulatory networks with emphasis on metabolism and development are the cross-pathway control of amino acid biosynthesis and the COP9 signalosome-dependent system, respectively.

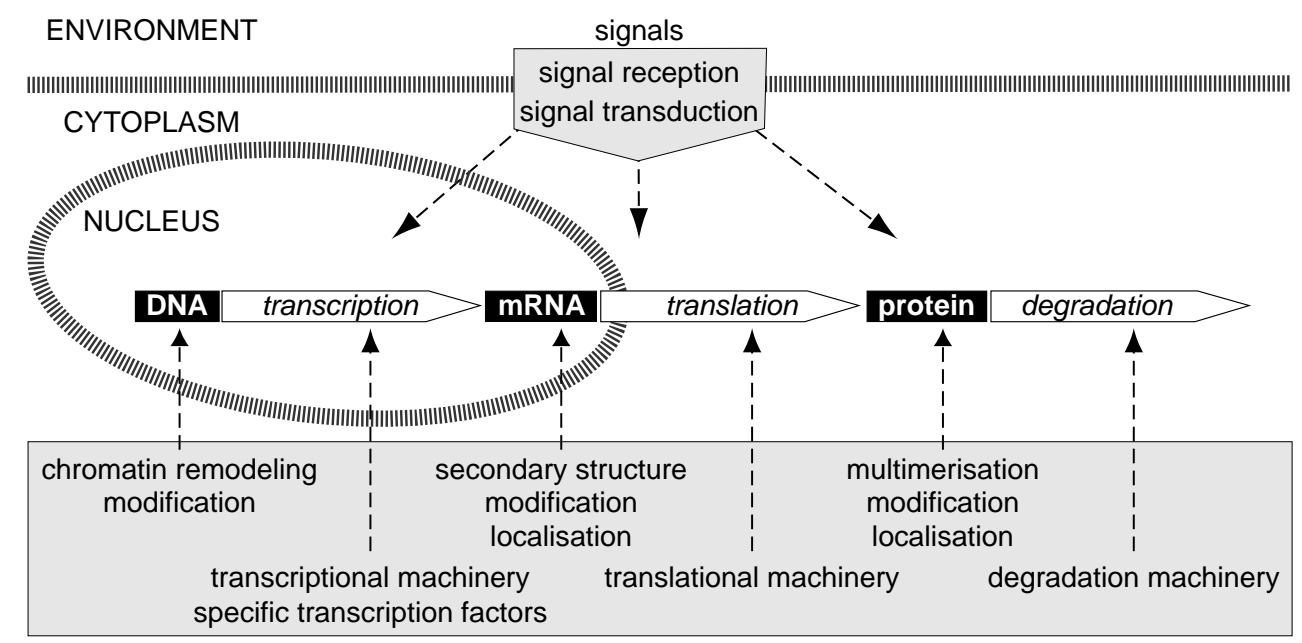

Fig. 1.1: Genetic control of protein synthesis and turnover in a eukaryotic cell. The scheme shows the central pathway of protein synthesis and degradation defining the molecular levels of regulatory attack. Grey fillings highlite examples for general mechanisms of molecular control with their target level indicated by broken-lined arrows.

\subsubsection{Expression of gene products and the cross-pathway control}

1.2.1.1 The yeast central transcriptional activator Gcn4p. Instantaneous response to environmental changes requires rapid transcriptional reprogramming. The main players that co-ordinate transcriptional activation of genes attributed to a common physiological trait are specific transcription factors. These regulatory trans-acting proteins bind to defined nucleotide motifs in the promoter of their target genes and recruit the transcriptional machinery for initiation of transcription. These two functions generally imply at least two domains characteristic for transcription 
A

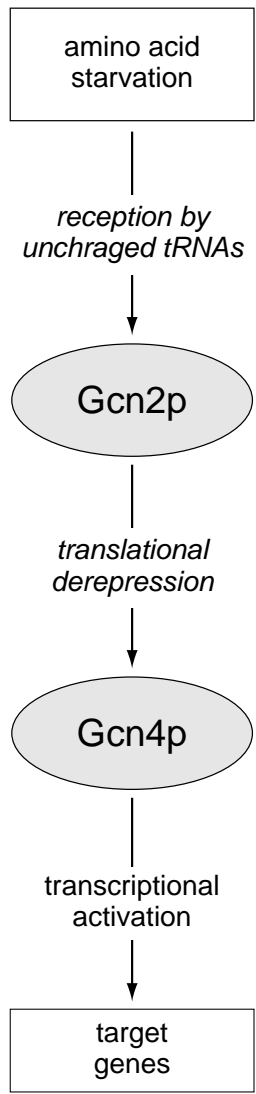

B

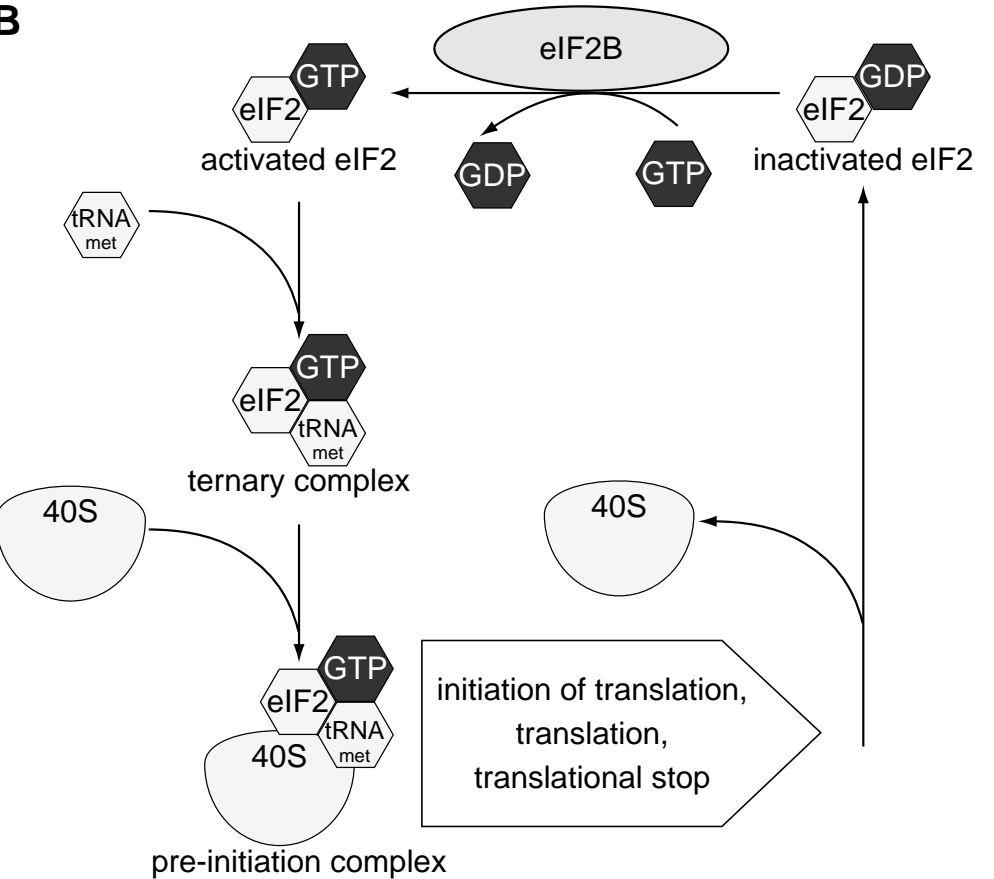

C

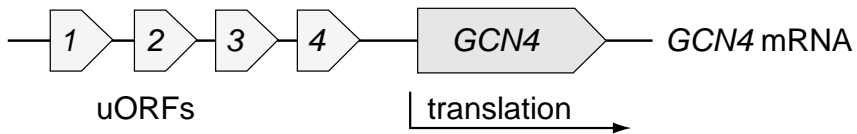

Fig. 1.2: Regulation of the synthesis of yeast Gcn4p transcriptional activity. (A) The signal "amino acid starvation" is perceived and transduced by the sensor kinase Gcn2p and finally results in translational derepression of the transcriptional activator Gcn4p which in turn activates transcription of corresponding target genes. (B) Of central meaning for the translational derepression mechanism is the regeneration of activated eukaryotic translation initiation factor 2 (elF2). GTP-activated elF2 and tRNAmet form a ternary complex that is enlarged with the small ribosomal subunit (40S) to the pre-initiation complex that is prerequisite for initiation of translation. After the translational stop, elF2 is released in the GDP-bound, inactive form and depends on the guanosine exchange factor elF2B to rebuild a ternary complex. Upon amino acid starvation, Gcn2p phosphorylates elF2 which prevents GDP exchange and thus slows the process of translational initiation down. (C) Pivot of the translational derepression mechanism are the four small upstream open reading frames (uORFs) proximal of the GCN4 open reading frame. The function of these uORFs in regulation of GCN4 translation is described in the text.

factors. Recognition of the target sequence is mediated by a DNA binding domain (BD) with bZIP, bHLH or Zn-finger motif whereas recruitment of the transcriptional machinery is mainly mediated by an activation domain (AD) composed of glutamine and asparagine-, glutamine-, proline-, serine and threonine- or alaninrich regions (Johnson and McKnight 1989; Klevit 1991). A sophisticated regulatory circuit controlled by a transcription factor is the regulation of amino acid biosynthesis in response to exogenous availability of amino acids, termed crosspathway control (CPC) in filamentous fungi and general amino acid control (GAAC) in the yeast Saccharomyces cerevisiae (Piotrowska 1980; Hinnebusch 1988). The central transcription factor of this regulatory network is thoroughly studied in yeast and is described there as Gcn4p. Its carboxy terminal basic HLH domain and the 
leucine zipper mediate dimerisation and DNA binding (Saudek et al. 1990; Pu and Struhl 1991; Ellenberger et al. 1992) to the general control responsive elements (GCRE) with the consensus sequence 5'-TGA C/G TCA-3' (Oliphant et al. 1989). The transcriptional activation domain is subdivided into an $\mathrm{N}$-terminal and a central acidic part, separated by a PEST instability region (Kornitzer et al. 1994; Drysdale et al. 1995; Jackson et al. 1996; Drysdale et al. 1998). Additionally, the amino acid sequence of Gcn $4 p$ contains two functional nuclear localisation sequences (NLS) that mediate the transport of the transcriptional activator into the nucleus, independent of amino acid availability (Pries et al. 2002).

Extensive microarray studies revealed that $14 \%$ of the yeast's genome responds transcriptionally to amino acid starvation in a proposed Gcn4p dependent manner (Natarajan et al. 2001). Many of the induced genes contain a GCRE within the 5'-proximal 300 nucleotides and thus are potential direct Gcn4p target genes. They include representatives of all amino acid or amino acid precursor biosynthesis pathways and other putative targets related to synthesis or uptake of amino acids. Nevertheless, several of the identified putative targets are not related to amino acid metabolism, including several transcription factors and other cellular regulators. Some genes were even repressed under amino acid starvation conditions, but since repression affected predominantly genes of ribosomal and translational genes of which most lack GCREs, the repression effect is probably indirect and confers to decreased ribosome production and protein synthesis at amino acid starvation, or to activation of specific repressors (Hinnebusch and Natarajan 2002). It is additionally noteworthy that starvation for purine (Rolfes and Hinnebusch 1993), glucose (Yang et al. 2000), high salinity (Goossens et al. 2001) or ultraviolett light (Engelberg et al. 1994) also induce the Gcn4p regulatory system.

1.2.1.2 Control of Gen4p expression. To enable rapid modulation of differentiated transcriptional levels of gene families in response to changes in the environment, several mechanisms limit abundance, distribution and activity of the corresponding transcription factors. Complex regulation of Gcn $4 p$ occurs on several molecular levels. Though amino acid starvation results in increased GCN4 mRNA levels, regulation of GCN4 transcription seems of only minor importance in yeast (Albrecht et al. 1998).

The major regulation of Gcn $4 p$ expression is a translational derepression mechanism that is activated through a signal transduction cascade upon the signal amino acid starvation (Fig. 1.2A). The molecular mechanism of this cross-pathway signal transduction cascade is explained in a widely accepted model (Hinnebusch 1997): Limitation of amino acids leads to accumulation of uncharged tRNAs in the cell that bind to the hisRS domain of the sensor kinase Gcn2p (Wek et al. 1995). The Gcn2p kinase domain subsequently transduces this signal to the eukaryotic translation initiation factor 2 (elF2) by phosphorylation (Qiu et al. 2001), which in the end leads to shortage of ternary complex and thus slowing down of translational initiation as described in Figure 1.2B. With the resulting low rate of translational initiation, the translational barrier mediated by four small upstream open reading 
frames (uORFs) in the GCN4 mRNA leader (Fig. 1.2C) can be overcome (Hinnebusch 1997). Upon non-starvation conditions, this barrier is mainly mediated by translation of UORF 4 after which about $50 \%$ of the 40 S ribosomal subunits dissociate from the mRNA and never reach the GCN4 open reading frame. Upon amino acid starvation, the $40 \mathrm{~S}$ ribosomal subunit resumes scanning the mRNA after translation of UORF1, but due to the shortage of ternary complex, the AUG start codon of the inhibitory UORF4 is passed by until a new pre-initiation complex is formed and translation re-initiates at the GCN4 translational start.

Additionally, interference of the general control to the nitrogen sensing system has been shown to act on the translational level (Grundmann et al. 2001). Though nitrogen deprivation increases GCN4 transcription, it represses its further expression. Thus, nitrogen starvation overrides the translational induction of Gcn4p but seems to keep higher GCN4 mRNA levels in order to rapidly induce translation when nitrogen sources become available.

1.2.1.3 Post-translational control of Gcn4p. Once Gcn4p is synthesised, its stability is carefully controlled. It is a short lived protein that is rapidly degraded under non-starvation conditions (Kornitzer et al. 1994). Prerequisite for degradation is phosphorylation of tyrosine residues in the activation domain of Gcn $4 p$ by at least two cyclin-dependent kinases, Pho85p and Srb10p (Meimoun et al. 2000; Chi et al. 2001) (Fig. 1.3A). The control of Gcn $4 p$ degradation, as well as localisation of Pho85p, is restricted to the nucleus (Pries et al. 2002). Phosphorylated Gcn4p is subsequently ubiquitinylated by the $\mathrm{SCF}^{\mathrm{Cdc} 4}$ ubiquitin ligase which targets it to degradation by the $26 \mathrm{~S}$ proteasome (see 1.2.2.1).

GCRE-bound Gcn $4 p$ is probably involved in the recruitment of the transcriptional machinery to the target promoter and can directly interact with at least three basal transcriptional co-activator complexes, TFIID, SAGA and MED (Drysdale et al. 1998; Natarajan et al. 1999). (i) The basal transcription factor of RNA polymerase II (TFIID) enables formation of the transcriptional pre-initiation complex. It is composed of the IATA-binding protein (TBP) that binds to promoters and several TBP associated factors (TAFs) that mediate e.g. protein kinase and acetyltransferase activity (Burley and Roeder 1996; Green 2000). TBP and the Gcn4p binding domain are connected by the co-activator multiprotein bridging factor (Mbf1p), that probably recruits the TBP to promoters where specific transcription factors are bound (Takemaru et al. 1998). (ii) The $\underline{S} p t / \underline{A} d a / \underline{G} c n 5$ acyltransferase complex (SAGA) is composed of multiple transcriptional regulatory proteins, including several TBP-related components and the histone acetlytransferase Gcn5p. It thus links chromatin modification and the basal transcription machinery with specific transcription factors (Grant et al. 1997; Drysdale et al. 1998; Winston and Sudarsanam 1998). (iii) The RNA polymerase II holoenzyme mediator complex (MED), composed of more than 20 proteins, directly interacts with components of the RNA polymerase II and acts as an essential coactivator. It mediates response of the transcriptional machinery to specific transcriptional activators at upstream DNA binding sequences by modulating RNA polymerase II activity (Kim et al. 1994; Hengartner et al. 1995). Strikingly, the Srb10p 
A
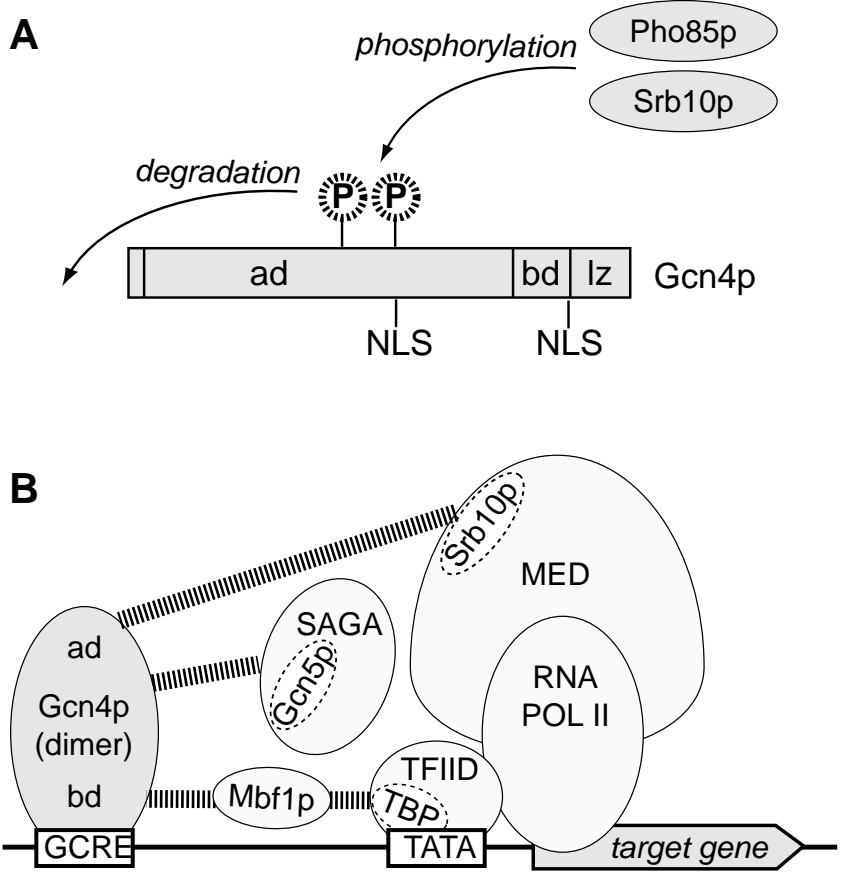

Fig. 1.3: Regulation of the turnover of yeast Gcn4p transcriptional activity. (A) Threonin residues in the Gcn4p activation domain (ad) can be phosphorylated by at least two different protein kinases, Pho85p and Srb10p. Phosphorylated Gcn4p is subject to ubiquitination by the E3 ligase SCFCdc4. Nuclear localisation motifs (NLS) are responsible for the transport of the transcription factor to its site of action: the nucleus. (B) Gcn4p binds as a dimer to GCRE elements in the target gene's promoter region and recruits the transcriptional machinery and accessory multiprotein complexes. Several interactions of Gcn4p with multiprotein complexes have been shown, this scheme summarises examples. RNA polymerase II (RNA POL II), the basal transcription factor IID (TFIID), the mediator complex (MED) and the Spt/Ada/Gcn5 acetyltransferase complex (SAGA). Direct protein-protein interactions are indicated by paralleled lines. In this scenario, Gcn4p is phosphorylated by Srb10 (a MED component) during its function as transcriptional activator. Abbreviations: activation domain (ad), DNA binding domain (bd), leucine zipper (lz).

protein kinase is associated with the large multiprotein mediator complex (Hengartner et al. 1995).

As mentioned above, Srb10p phosphorylates Gcn4p and thus targets it to the degradation machinery (Chi et al. 2001). This implies that promoter-bound Gcn4p specifically and probably independently recruits TFIID, SAGA and the MEDPolymerase II-holoenzyme to the promoter, enables initiation of transcription and is in turn specifically selected for proteolysis by phosphorylation. Such an interdependency of transcription factor stability and transcriptional machinery may prevent that a single promoter bound molecule activates multiple rounds of transcription initiation and thus restricts the global disruption of transcriptional control that results from squelching (Tansey 2001).

1.2.1.4 The cross-pathway control of $\boldsymbol{A}$. nidulans. The cross-pathway control in filamentous fungi corresponds to the general control in yeast and is subject to intensive research. Homologues of yeast GCN4 have been identified in the filamentous fungi Neurospora crassa (Paluh et al. 1988) and A. niger (Wanke et al. 1997) sharing in their deduced sequences high amino acid sequence identities with the mammalian oncoprotein c-jun (Bohmann et al. 1987). During the course of this work, the corresponding $A$. nidulans cross-pathway control transcriptional 
activator gene $c p c A$ was isolated. It is functionally exchangeable with the yeast Gcn4p, indicating that the system that mediates amino acid starvation response is highly conserved between yeast and filamentous fungi (Hoffmann et al. 2001). Accordingly, nucleotide motifs that resemble the yeast GCRE consensus sequence are found in the promoter region of $A$. nidulans genes that are activated by CPCA. Examples are $\arg B$ (Piotrowska 1980) and two additional genes identified during the course of this work: $\operatorname{trpB}$ (Eckert et al. 2000) and hisHF (Valerius et al. 2001). Correspondingly, this consensus is termed cross-pathway response element (CPRE) in filamentous fungi. Examples like aroC (Krappmann et al. 1999) show that not all genes acting in amino acid metabolism are regulated by the crosspathway control and, vice versa, CPCA-mediated transcriptional activation of the functionally yet uncharacterised putative DNA binding protein jlbA (Strittmatter et al. 2001) indicates that not solely genes involved in amino acid metabolism are regulated by the cross-pathway control in $A$. nidulans. The $A$. nidulans crosspathway transcriptional activator seems to be regulated in a similar complex but not identical manner as its yeast counterpart. Only two uORFs mediate translational de-repression of the $A$. nidulans $c p c A$ mRNA. Additionally, CPCA significantly autoregulates its own transcription by CPREs in its promoter, as it is also seen in mammalian jun and fos genes but not in the yeast GCN4 gene (Hoffmann et al. 2001).

\subsubsection{Degradation of gene products and the COP9 signalosome.}

1.2.2.1 Ubiquitin dependent protein degradation. Controlled timely destruction of proteins is a major regulatory mechanism of complex cellular processes. The main eukaryotic protein degradation machinery, the $26 \mathrm{~S}$ proteasome, specifically degrades ubiquitinylated proteins (Fig. 1.4A). Proteolysis is performed by the ATPindependent peptidase activity of the proteasome $20 \mathrm{~S}$ core particle, whereas substrate specificity is mediated by the $19 \mathrm{~S}$ regulatory particle composed of a base complex that confers ATPase and chaperon activity and the 26S lid complex (LID) with yet unknown mechanism. Substrates are targeted for degradation by conjugation of ubiquitin, a small protein of 111 amino acids with catabolic role when added as polyubiquitin chains. The corresponding ubiquitinylation cascade (Fig. 1.3B) is extensively studied and thoroughly reviewed (Hershko and Ciechanover 1998; Jackson and Eldridge 2002). An ubiquitin activating enzyme (E1) activates ubiquitin by thioester linkage prior to transfer to a lysine residue of a specific protein substrate by an ubiquitin conjugating enzyme (E2). An ubiquitin ligase (E3) catalyses substrate recognition and assists in ubiquitinylation.

Several E3 ubiquitin ligase complexes are known, most prominent is the cullin-RING-H2-family that includes SCF ( $\underline{\text { Skp1/C }}$ dc53/F-box) complexes (Deshaies 1999; Tyers and Jorgensen 2000). These E3 ubiquitin ligases are generally composed of four major subunits. The core activity is performed by a cullin (CUL1) and a RING-H2 domain protein (HRT1/ROC1/RBX1). Both are associated together with the SKP1 subunit to a special F-box protein as substrate receptor. Different E3 
A

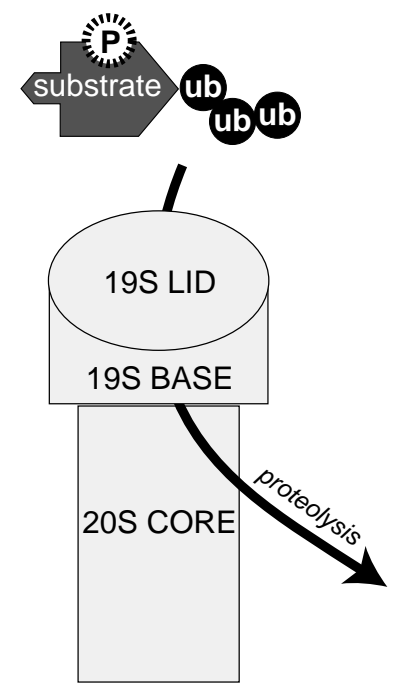

B

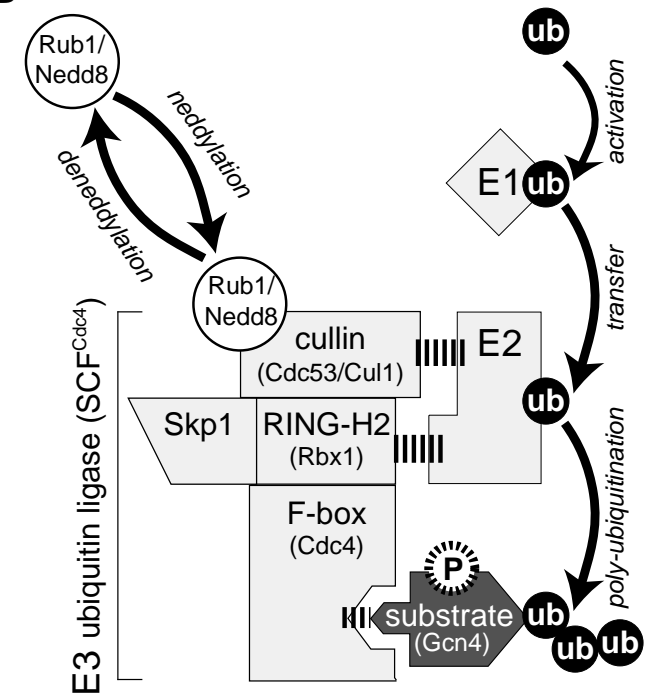

Fig. 1.4: Ubiquitin dependent protein degradation. A schematic overview of the major multiprotein complexes involved in ubiquitin-dependent proteolysis is given, modified from Tyers and Jorgensen (2000) and von Arnim (2001). (A) The 26S proteasome specifically degrades ubiquitinylated proteins. (B) Ubiquitination of the target substrates follows an enzymic cascade of an ubiquitin activating enzyme (E1), and ubiquitin conjugating enzyme (E2) and an ubiquitin ligase (E3). The four critical subunits of a typical E3 ligase are shown, with the yeast protein nomenclature for the E3 SCFCdc4 that targets Gcn4p for degradation given in parentheses. Parralelled lines indicate protein-protein interaction.

ubiquitin ligase complexes are designated by their accessory F-box subunit. Since protein degradation involves regulation of essential cellular processes, the targeting of substrates to ubiquitin-dependent degradation by the $26 \mathrm{~S}$ proteasome must be tightly connected to the actual cellular response signalling pathways. SCF ubiquitinylation activity towards potential target substrates is regulated by at least two mechanisms, phosphorylation and neddylation. (i) SCF activity towards the stability of its substrates depends on the level of substrate phosphorylation by the corresponding protein kinases. Exemplary for Gcn4p (see 1.2.1), ubiquitinylation by $\mathrm{SCF}^{\mathrm{CDC} 4}$ requires phosphorylation by Pho85 or Srb10 (Meimoun et al. 2000). (ii) The ubiquitinylation activity of the SCF itself can be modulated by reversible conjugation of the ubiquitin-related protein NEDD8/Rub1 on the cullin subunit, a process called neddylation. Cycling of neddylation and deneddylation are essential for E2-E3 complex formation (Kawakami et al. 2001) and cullin-dependent polyubiquitinylation of SCF target proteins (Morimoto et al. 2000; Podust et al. 2000; Read et al. 2000; Wu et al. 2000; Ohh et al. 2002). Proteins with specific neddylation activities were identified (del Pozo et al. 2002; Fan et al. 2002), but a distinct deneddylase was not isolated yet.

1.2.2.2 The COP9 signalosome. A regulatory complex tightly connected to the control of ubiquitin dependent protein degradation is the constitutive photomorphogenesis $\underline{9}$ complex (COP9) termed $\underline{\text { COP9 }}$ signalosome (CSN). Together with the 19S proteasome lid (LID) and the eukaryotic translation initiation factor $\underline{3}$ (elF3), the CSN belongs to the $\mathrm{PCl}$ family of multiprotein complexes that is highly conserved among higher eukaryotes. Their subunits are characterised by either a PCl (proteasome, $\underline{\mathrm{COP}}$, elF3) or a MPN (Mpr1p, Pad1 $\underline{\mathrm{N}}$-terminal) domain 
of about 200 and 140 amino acids, respectively. These domains might mediate stabilisation of protein-protein interactions, though their precise function remains obscure (Glickman et al. 1998; Hofmann and Bucher 1998; Kim et al. 2001). The COP9 signalosome (Fig. 1.5A) is composed of the eight subunits CSN1-CSN8 (Deng et al. 2000). These exhibit several subunit-subunit and non-subunit interactions (Tab. 1.1) and can partially be phosphorylated (Kapelari et al. 2000; Bech-Otschir et al. 2002). The proteasomal lid shows a similar subunit composition with Rpn7, 6, 3, 5, 11, 8, 9 and 12 corresponding to CSN1-8 (Kim et al. 2001). But electron microscopy of subunit arrangements revealed that the overall architecture of CSN and LID is not identical, though at least both share an asymmetric shape enclosing a central cave (Kapelari et al. 2000; Fu et al. 2001). Both complexes share a similar size of about $500 \mathrm{kDa}$ and are localised mainly in or close to the nucleus. A common evolutionary origin of the $\mathrm{PCl}$ complexes is thus discussed, with elF3 being more distantly related (Hofmann and Bucher 1998; Kapelari et al. 2000; Fu et al. 2001; Kim et al. 2001; Tsuge et al. 2001).

Tab. 1.1: Seletion of interactions of mammalian COP9 signalosome subunits

\begin{tabular}{|c|c|c|c|}
\hline \multicolumn{2}{|l|}{ protein } & \multicolumn{2}{|c|}{ nteraction / CSN-mediated activity ${ }^{1}$} \\
\hline \multicolumn{4}{|c|}{ degradation machinery: } \\
\hline $\begin{array}{l}\text { CUL1 } \\
\text { Rpt6 }\end{array}$ & $\begin{array}{l}\text { SCF cullin subunit } \\
26 S \text { proteasome LID subunit }\end{array}$ & $\begin{array}{l}\text { CSN2 / D } \\
\text { CSN3 }\end{array}$ & $\begin{array}{l}\text { Lyapina et al. } 2001 \\
\text { Kwok et al. } 1999\end{array}$ \\
\hline \multicolumn{4}{|c|}{ regulatory proteins: } \\
\hline $\begin{array}{l}\text { Bcl3 } \\
\text { c-Jun } \\
\text { ICSBP } \\
\text { IKK } \gamma \\
\text { LFA-1 } \\
\text { MIF } \\
\text { p27 } \\
\text { p53 } \\
\text { PR } \\
\text { rLHR } \\
\text { SCR-1 } \\
\text { THR } \\
\text { Vpr }\end{array}$ & $\begin{array}{l}\text { IאB multigene family member } \\
\text { transcription factor / AP-1 } \\
\text { interferon consensus sequence binding protein } \\
\text { IאB-kinase complex component } \\
\text { integrin } \\
\text { cytokine macrophage migration inhibitory factor } \\
\text { cyclin-dependent kinase inhibitor } \\
\text { tumor suppressor } \\
\text { progesteron receptor } \\
\text { lutropin/choriogonadotropin receptor precursor } \\
\text { steroid receptor co-activator } \\
\text { thyroid hormone receptor } \\
\text { HIV-1 accessory protein }\end{array}$ & $\begin{array}{l}\text { CSN5 / + } \\
\text { CSN5 / + P } \\
\text { CSN2 / + P } \\
\text { CSN3/- } \\
\text { CSN5 } \\
\text { CSN5 } \\
\text { CSN5 / - } \\
\text { CSN5 / - } \\
\text { CSN5 / + } \\
\text { CSN5 / - } \\
\text { CSN5 / + } \\
\text { CSN2 } \\
\text { CSN6 }\end{array}$ & $\begin{array}{l}\text { Dechend et al. } 1999 \\
\text { Naumann et al. } 1999 \\
\text { Cohen et al. } 2000 \\
\text { Hong et al. } 2001 \\
\text { Bianchi et al. } 2000 \\
\text { Kleemann et al. } 2000 \\
\text { Tomada et al. } 1999 \\
\text { Bech-Otschir et al. } 2001 \\
\text { Chaucherau et al. } 2000 \\
\text { Li et al. } 2000 \\
\text { Chaucherau et al. } 2000 \\
\text { Lee et al. } 1995 \\
\text { Mahalingam et al. } 1998\end{array}$ \\
\hline
\end{tabular}

${ }^{1} \mathrm{CSN}-$ mediated stability (+) or proteolysis (-) effects and proven phosphorylation (P) or deneddylation (D) activities are indicated.

The available data on CSN function suggest that it contributes to the regulation of ubiquitin dependent degradation by modification of E3 ubiquitin ligase activity by at least two different functions (Seeger et al. 2001; Bech-Otschir et al. 2002). The COP9 signalosome promotes deneddylation of cullins by a proposed associated isopeptidase that mediates deneddylase activity, as shown for mammalian cells, A. thaliana and S. pombe (Zhou et al. 2001; Wang et al. 2002; Yang et al. 2002). Additionally, an associated Ser/Thr kinase activity of the CSN phosphorylates proteins like p53 (Bech-Otschir et al. 2001), c-Jun (Musti et al. 1997; Naumann et al. 1999) and ICSBP (Cohen et al. 2000) and thus alters their susceptibility to ubiquitinylation. The precise mechanisms of the deneddylation and phosphorylation activities associated with CSN and the connections between both are not yet understood. To date, two major working hypotheses for the CSN 

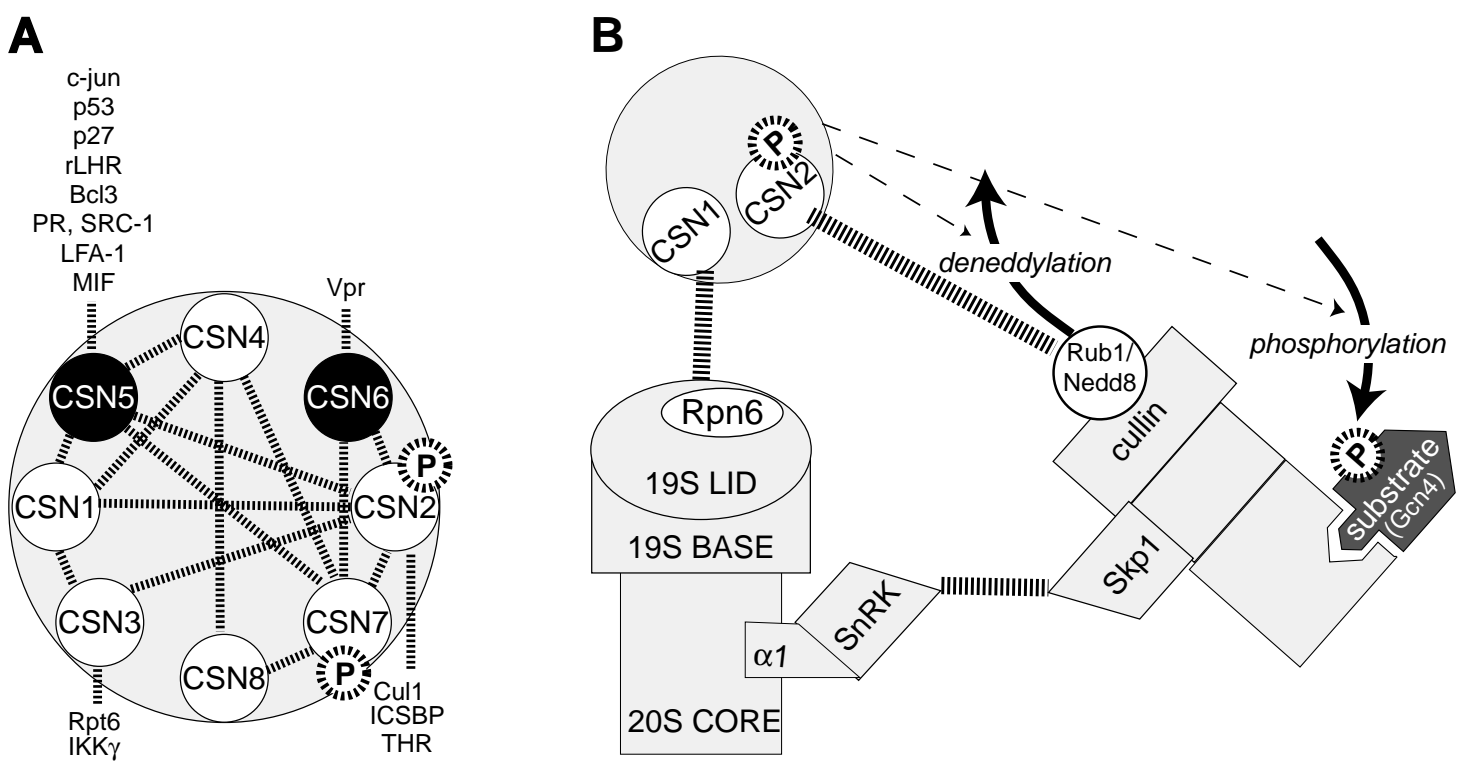

Fig. 1.5: The COP9 signalosome (CSN) and its proposed functions. Protein-protein interaction are indicated by parralelled bars. (A) The eight subunits of the CSN are interconnected by several protein-protein interactions, and single subunits additionally interact with non-subunit proteins (see also Tab. 2) as summarised in Kappellari et al., 2000 and Bech-Otschir et al., 2002. (B) Several ineractions between proteasome, SCF and CSN suggest physical association of the three multiprotein complexes (Kwok et al.1999, Schwechheimer et al. 2001; Farras et al. 2001). Additionally, the CSN-associated functions are indicated by broken arrow lines.

associated activities have been suggested. One model relies on the interaction between CSN and the $26 \mathrm{~S}$ proteasome, since subunits of both complexes co-purify (Seeger et al. 1998) and specific protein-protein interactions were identified between CSN and subunits of the proteasome regulatory complex (Kwok et al. 1999). The CSN might thus act as an alternative or additional proteasome lid or modulate proteasome activity by altering the activity of the lid complex (Wei and Deng 1999; Schwechheimer and Deng 2000). A second model suggests the CSN as a platform with scaffolding function for associated enzymes like kinase(s), deneddylase(s), and E3 ubiquitin ligase(s) (Bech-Otschir et al. 2002). More precisely, the CSN was suggested to directly interact with SCF type 3 ubiquitin ligases or associate with the complete E2-E3 complex to modulate E3 activity (Schwechheimer and Deng 2001; Bech-Otschir et al. 2002; Suzuki et al. 2002). Notably, also the 26S proteasome and the E2-E3 complex interact, which implies that the processes of ubiquitinylation and proteolysis are probably not separated. The Snf1-like protein kinase SnRK interacts with the $\alpha 4$ subunit of the proteasome core complex and with the Skp1 subunit of the SCF and might thus constitute a proteasomal docking station for the SCF. This led to a model which proposes that the SCF changes between proteasome associated and a free status (Tongaonkar et al. 2000; Verma et al. 2000; Xie and Varshavsky 2000; Farras et al. 2001; von Arnim 2001). Taken together (Fig. 1.5B) it seems conceivable that further studies will reveal complex and non-statically physical associations between the three multiprotein complexes $26 \mathrm{~S}$ proteasome, E3 ubiquitin ligases and the COP9 signalosome. 
1.2.2.3 Physiological role of the COP9 signalosome. The targets of the COP9 signalosome include a variety of proteins, particularly transcription factors, cell cycle regulators and hormone receptors (Tab. 1.1). Physiologically, the CSN is thus involved in the regulation of several processes predominately affecting cellular homeostasis, growth, differentiation and development. Correspondingly, defects in CSN function display a pleiotrophic mutant phenotype higher eukaryotes. In plants and insects, a functional COP9 signalosome is essential for survival: though CSN mutants successfully pass embryogenesis, they die during later developmental stages. This post-embryonic lethality becomes evident in CSN mutant seedlings of the plant $A$. thaliana that cease development after a few days (Wei et al. 1994). Comparably, larvae of the invertebrate $D$. melangolaster stop differentiation after pupation (Freilich et al. 1999). Thus, mutant analyses are restricted to the first days of growth and development or to reduced-function mutants.

The model organism for studies concerning the COP9 signalosome is A. thaliana, where mutations in CSN subunits result in the loss of complex formation and pleiotrophic mutant phenotypes (Wei and Deng 1999). With light and auxin responses, two major target pathways of CSN regulation have been described thoroughly. (i) Light response includes the integration of a physical parameter into regulation of development. Photomorphogenesis of plant seedlings proceeds in the light but is repressed in the dark. In A. thaliana, the corresponding light signal transduction depends on ubiquitin-dependent degradation of HY5, the transcriptional activator of light regulated genes (Osterlund et al. 2000). At least three constitutive photomorphogenesis (COP) complexes are involved in this process: the CSN, COP1 and COP10 (Ang et al. 1998; Schwechheimer and Deng 2000). It is assumed that they define a protein ubiquitinylation pathway that directs the degradation of HY5 in the dark (Suzuki et al. 2002). COP1, that accumulates in the nucleus in the dark, probably functions as E3 ubiquitin ligase (Osterlund et al. 1999; Osterlund et al. 2000), COP10 might mediate the corresponding E2 activity. The COP9 signalosome probably contributes to the ubiquitinylation process by its associated activities as E3-modulator (Suzuki et al. 2002). (ii) Auxin response describes the integration of a chemical parameter into regulation of differentiation. Plant response to the phytohormone auxin is responsible for a number of developmental processes like proper cell elongation and suppression of secondary inflorescences. In $A$. thaliana it is mediated by expression of the AUXIAA genes encoding short-lived transcriptional repressor proteins that are supposed substrates of the E3 ubiquitin ligase SCF ${ }^{\text {TIR1 } 1}$ (Abel et al. 1994; Gray et al. 2001). The CSN deneddylates the cullin of SCF ${ }^{T / R} 1$ and thus probably diminishes auxin signalling by degradation of the AUXIAA repressors (Schwechheimer et al. 2001). Moreover, the studies on auxin responses support the model of tight association between proteasome and E3 ligase (Fig. 1.5B). The regulatory WD40 protein PRL1 interacts with SnRK and might thus control docking of the SCF to the proteasome by attenuation of SnRP binding. It seems striking that, in respects to auxin response, PRL1 mutations cause the opposite effect of CSN mutations in A. thaliana, suggesting that proteosomal binding and deneddylation of the SCF are essential for proper auxin response (Nemeth et al. 1998; Gray and Estelle 2000; 
Farras et al. 2001).

The CSN subunits were identified in several vertebrate tissue cells including mouse and human (Wei and Deng 1998; Bounpheng et al. 2000). They are biochemically related to the ubiquitinylation system in regulating stability of transcription factors and cell cycle regulators. They interact with cullin subunits (Lyapina et al. 2001; Yang et al. 2002) and are probably also involved in developmental processes. One mammalian CSN target is of major interest, because of an implication in tumor growth. This is the transcription factor c-Jun, the mammalian homologue of yeast Gcn4p, that increases specificity of target gene activation by AP-1 proteins through c-Jun / AP-1 site complex stabilisation (Claret et al. 1996). Because of its short half life, c-Jun protein levels are constitutively low. cJun half-life is increased when it is phosphorylated by mitogen- and stress activated Jun-terminal kinases (JNK), since phosphorylation prevents ubiquitindependent degradation of C-Jun (Karin et al. 1997). The CSN stabilises C-Jun towards the ubiquitin system by phosphorylation independent of the known JNK pathway (Naumann et al. 1999; Chamovitz and Segal 2001). This CSN-directed CJun stabilisation results in increased AP-1 activity which in turn affects expression of regulatory proteins like the vascular endothelial growth factor (VEGF) that controls tumor angiogenesis (Pollmann et al. 2001). Additionally, the c-Jun/p53 balance - that is disturbed in many tumor cells - is at least partially controlled by the CSN mediated ubiquitin system. Both proteins directly interact with CSN5 for CSNmediated phosphorylation, with c-Jun being stabilised (Musti et al. 1997) and p53 destabilised upon phosphorylation (Bech-Otschir et al. 2001).

1.2.2.4 The COP9 signalosome in lower eukaryotes. The COP9 signalosome, well conserved in higher eukaryotes, was not identified in any of the prokaryotic genome sequences known to date. Knowledge about the COP9 signalosome in lower eukaryotes is scarce. The complete genome sequence of the baker's yeast $S$. cerevisiae revealed with the open reading frame of $R R / 1$ only one putative coding region with similarity to the COP9 signalosome subunit 5 . Subunits CSN1CSN6 were identified in S. pombe (Mundt et al. 1999; Peng et al. 2001b; Peng et al. 2001a; Zhou et al. 2001; Mundt et al. 2002), all of which are essential for proper cullin deneddylaton suggesting the existence of the complete complex in fission yeast. S. pombe csn mutant strains show less severe defects than their metazoan counterparts. Moreover, deletion of various subunits revealed different mutant phenotypes. Loss of CSN1 resulted in a cell cycle defect, slow growth with reduced cell elongation and increased UV sensitivity, whereas deletion of other CSN subunits did not display these mutant phenotypes. Distinct functions for different CSN subunits of fission yeast were proposed. A COP9 signalosome is not yet identified in filamentous fungi. In A. nidulans, few components of an ubiquitinrelated protein degradation machinery are yet described: two ubiquitin genes (Noventa-Jordao et al. 2000) and two open reading frames encoding a putative cullin and a putative CSN4-like component (Eckert 2000), respectively. Additionally, the pre-competence gene $a c o B$ shows similarity to $C S N$ subunit 7 , though overall amino acid identities are rather weak (Lewis and Champe 1995). 


\subsection{Regulation of metabolism in A. nidulans}

Metabolism defines the sum of biosynthetic processes occurring within a living organism (Fig. 1.6). The division between primary and secondary metabolism is rather artificial and boundaries are blurred. Generally, primary metabolism is defined to be essential since it uses energy from nutritional sources to ensures production of cellular components. Contrary, secondary metabolites comprise a variety of complex, according to the definition non-essential organic compounds that are normally produced when growth ceases (Firn and Jones 2000). Cascades of enzymatic reactions define metabolic pathways that lead to the biosynthesis of a specific metabolite. Such pathways are often not linear since substrates, intermediates and products are often shared for production of different metabolites. Thus, metabolism can be regarded as an interconnected network of enzymatic activities that are modulated according to the immediate situation of the cell. This implies highly sophisticated regulatory systems that control production of various metabolites in response to the environmental conditions. Since the A. nidulans genome sequence is not yet fully open to the research community, the complete metabolic properties of this fungus remain to be discovered. Several regulatory proteins that impact metabolic pathways have already been described, though overall knowledge about their role in the whole cellular composition is rather fragmentary.

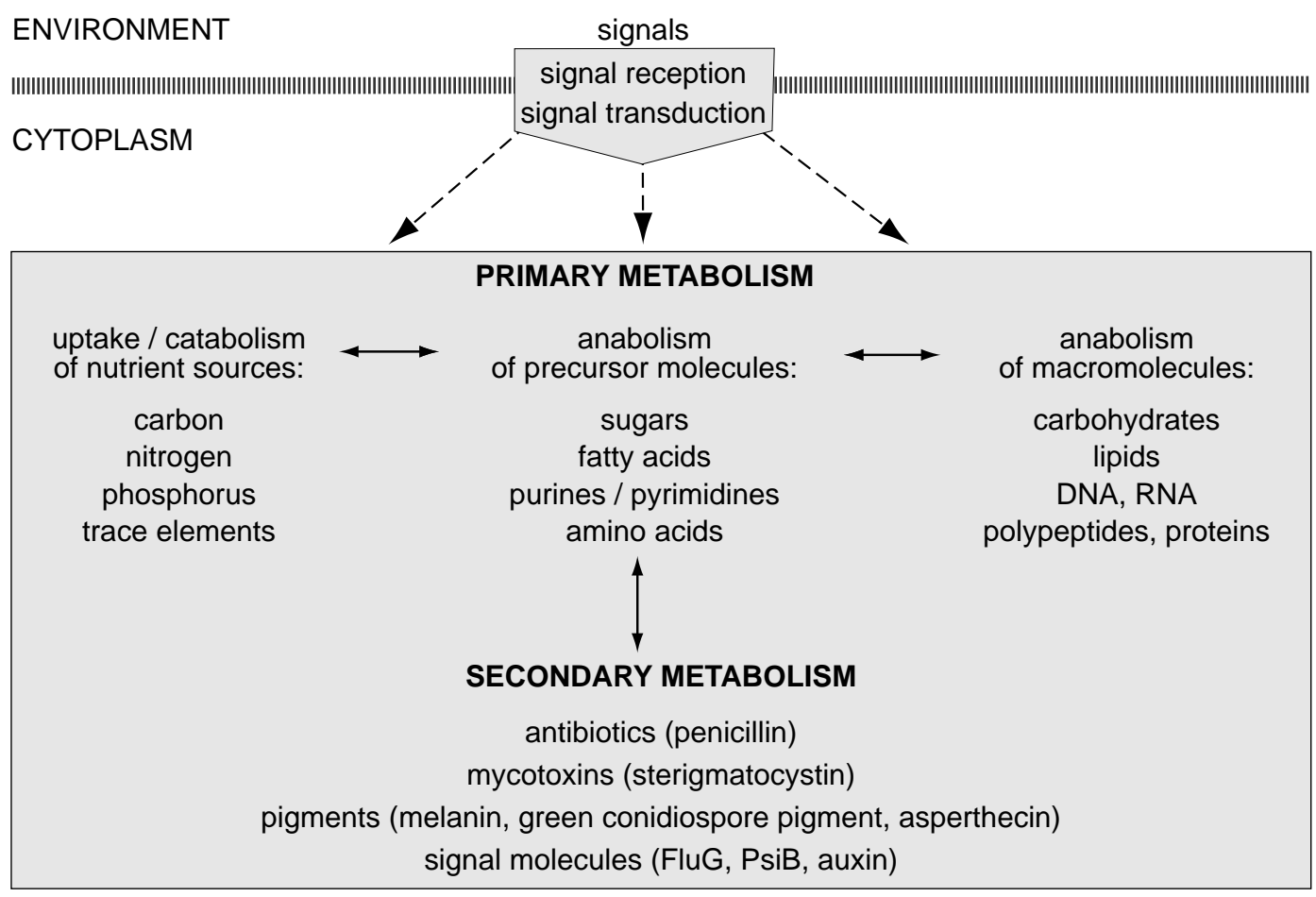

Fig. 1.6: Fungal metabolism. Enzyme activities of primary metabolism are modulated according to the availability of nutrient sources. Primary metabolites serve as precursor molecules for secondary metabolites. Dashed arrow lines indicate the dependency of metabolism on environmental signals. 


\subsubsection{Primary metabolism}

1.3.1.1 Nutrition. A. nidulans can use various nutrient sources and tolerates a broad $\mathrm{pH}$ spectrum, especially in the acidic region where numerous prokaryotic microorganisms have difficulties to survive (Pontecorvo et al. 1953). Expression of metabolic enzymes is restricted to their need in order to minimise energetic expenses, and several specific transcription factors are known to regulate metabolic traits in A. nidulans (Tab. 1.2). The major transcription factor of $\mathrm{pH}$ signalling in $A$. nidulans is PACC, ensuring that genes are expressed at a $\mathrm{pH}$ where the corresponding enzymes can function (Espeso and Penalva 1996). Utilisation of easily accessible nutrients at low energetic expense is normally preferred. Thus, uptake systems as well as the corresponding metabolic pathways are co-ordinately controlled by transcription factors of global metabolite repression

Tab. 1.2: Selection of important $A$. nidulans regulatory factors

\begin{tabular}{|c|c|c|}
\hline regulator $^{1}$ & regulatory circuit & references \\
\hline \multicolumn{3}{|c|}{ transcription factors: } \\
\hline ABAA & asexual development & Andrianopoulos and Timberlake 1994 \\
\hline ALCR & ethanol utilisation & Felenbok et al. 1988 \\
\hline AMDR & acetamide utilization & Andrianopoulos and Hynes 1990 \\
\hline AnCF & unknown & Steidl et al. 1999 \\
\hline AFLR & sterigmatocystein synthesis & Yu et al. 1996a \\
\hline AREA & ammonium repression & Punt et al. 1995 \\
\hline AREB & ammonium repression & Conlon et al. 2001 \\
\hline BRLA & asexual development & Prade and Timberlake 1993 \\
\hline CPCA & amino acid control & Hoffmann et al. 2001 \\
\hline CREA & glucose repression & Panozzo et al. 1998 \\
\hline DOPA & growth and development & Pascon and Miller 2000 \\
\hline MEDA & developmental modifier & Busby et al. 1996 \\
\hline NIR & inorganic nitrogen assimilation & Burger et al. 1991 \\
\hline NSDD & sexual development & Han et al. 2001 \\
\hline PACC & $\mathrm{pH}$ regulation & Espeso et al. 1997 \\
\hline SREA & iron uptake & Haas et al. 1999 \\
\hline STEA & sexual development & Vallim et al. 2000 \\
\hline STUA & developmental modifier & Dutton et al. 1997 \\
\hline UAY & purine utilization & Suarez et al. 1995 \\
\hline $\begin{array}{l}(\text { VEA) } \\
(\text { WFTA) }\end{array}$ & developmental light response & Mooney and Yager 1990 \\
\hline (VVEIA) & conidiosporogenesis & Marshall and Timberlake 1991 \\
\hline \multicolumn{3}{|c|}{ signal transduction: } \\
\hline FADA & sterigmatocystin, penicillin (G $\alpha$ - protein) & Tag et al. 2000 \\
\hline FLBA & growth, dev. (regulator of G-protein) & Yu et al. 1996b \\
\hline MPKA & polarised growth (mitogen-activated kinase) & Bussink and Osmani 1999 \\
\hline NIMA & cell cycle (kinase) & Lu et al. 1993 \\
\hline NIMO & cell cycle (kinase) & James et al. 1999 \\
\hline PCLA & cell cycle (cyclin) & Schier et al. 2001 \\
\hline PHOA & phosphorus, dev. (cyclin-dep. kinase) & Bussink and Osmani 1998 \\
\hline PPHA & hyphal growth ( $2 A$ protein phosphatase) & Kosmidou et al. 2001 \\
\hline RASA & carbon, dev. (small G protein) & Som and Kolaparthi 1994 \\
\hline SFAD & sterigmatocystin, penicillin (G $\beta$ - protein) & Rosen et al. 1999 \\
\hline
\end{tabular}

${ }^{1}$ Parentheses indicate that the function as transcription factor is conceivable but questioned.

Abbreviation: development (dev.). 
systems. The carbon catabolite repression system relies on the central transcriptional repressor CREA (Strauss et al. 1999) that blocks expression of enzymes for alternative carbon source consumption as long as glucose is available. An analogous mechanism mediated by the transcriptional repressor AREA (Platt et al. 1996) ensures that ammonium and glutamine are favoured as nitrogen sources. Additionally, the transcription factor SREA (Haas et al. 1999) specifically regulates transcription of genes for siderophore biosynthesis and iron uptake.

1.3.1.2 Amino acid metabolism. An important group of precursors for all proteins that are produced during primary metabolism are amino acids. Fungi are generally capable of the de novo synthesis of all 20 proteinogenic amino acids. Amino acids are not only building blocks for proteins but also serve as precursor molecules of other primary and secondary metabolites. Their production thus interconnects several pathways. An example is the tight link to carbohydrate metabolism: amino acids are anabolised from intermediates of glycolysis, pentosephosphate cycle or citric acid cycle and, vice versa, can often be catabolised into such intermediates. According to the carbon compound they originate from, amino acids are subdivided into 6 biosynthesis families which implies that the corresponding biosynthesis pathways are branched. One example is production of the aromatic amino acids (Fig. 1.7A) that share chorismate as last common key intermediate (Bentley 1990). Additionally, pathway intermediates are shared between biosynthesis of amino acids and other primary metabolites. Exemplary, biosynthesis of histidine (Fig. 1.7B) is not directly connected to the production of other amino acids but to de novo synthesis of purine via the by-product 5'-phosphoribosyl-4-carboxamide-5aminoimidazole (AICAR) (Alifano et al. 1996). Additionally, amino acids are precursors for a variety of secondary metabolites. A direct connection of primary and secondary metabolism is represented by the non-proteinogenic amino acid $\alpha$ aminoadipate (Fig. 1.7C) that constitutes a branch point of fungal lysine and penicillin biosynthesis (Brakhage 1998).

Only few components of amino acid biosynthesis pathways have been isolated from $A$. nidulans to date, and their regulation is subject to current research. The co-ordinated transcriptional activation of the $\arg B$ (Goc and Weglenski 1988), trpB (Eckert et al. 2000) and hisHF (Valerius et al. 2001) amino acid biosynthesis genes in response to external amino acid availability is mediated by the transcriptional activator CPCA of the cross-pathway control (Hoffmann et al. 2001), which corresponds to yeast Gcn4p. Knowledge about the mechanism of amino acid specific regulation and their biochemical connections to other pathways of primary and secondary metabolism in A. nidulans is scarce. Selected examples are given in Fig. 1.7: (i) On the transcriptional level, the hisHF gene encoding the branch point enzyme of histidine biosynthesis and AICAR cycle (heterodimeric imidazole-glycerole-phosphate synthase; E.C. 2.4.2.14 and E.C. 4.3.2.4) is not repressed by histidine but by adenine (Fig. 1.7B) (Valerius et al. 2001). (ii) On protein level, regulation by the pathways end-product has been shown for the aroCencoded chorismate mutase (E.C. 5.4.99.5) that is allosterically regulated with 
A

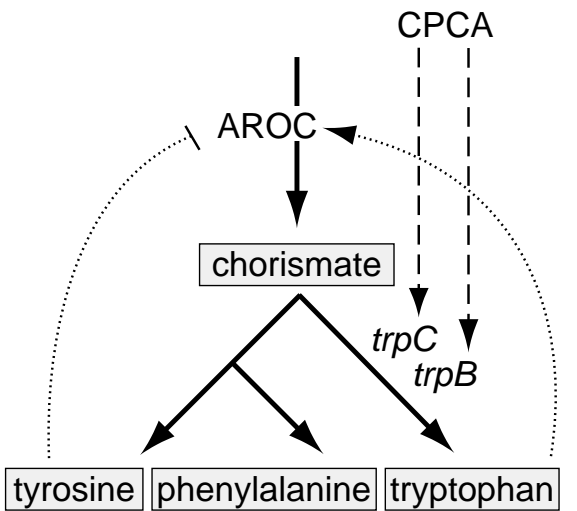

tyrosine phenylalanine tryptophan

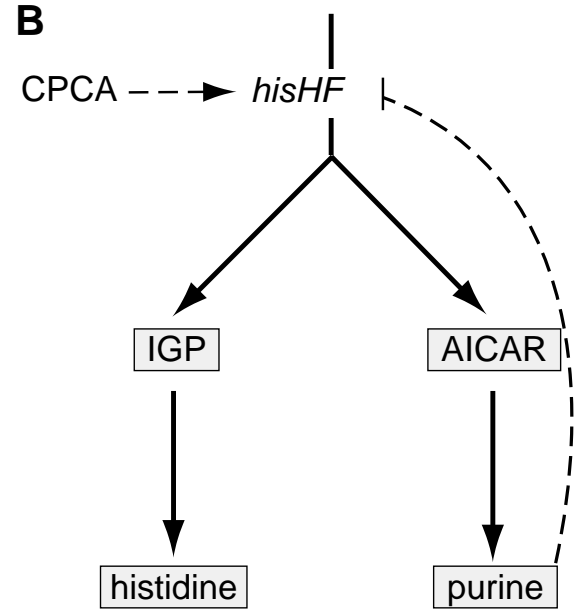

Fig. 1.7: Regulation of selected examples of amino acid biosynthesis in $A$. nidulans. The scheme shows cross-connections of amino acid biosynthesis with other pathways of primary and secondary metabolism. The corresponing amino acid biosynthesis genes isolated of $A$. nidulans and their known regulations are indicated. Regulation on transcriptional level is indicated by broken arrow lines, on protein level by dotted ones. See Table 1.2 for the regulators. (A) Aromatic amino acids share chorismate as precursor molecule in a branched biosynthesis pathway. (B) The biosynthesis of two primary metabolites, histidine and purine, is linked by AICAR. (C) A direct connection of primary and secondary metabolism constitutes the condension of $\alpha$-aminoadipate, valine and cysteine for penicillin production.

C

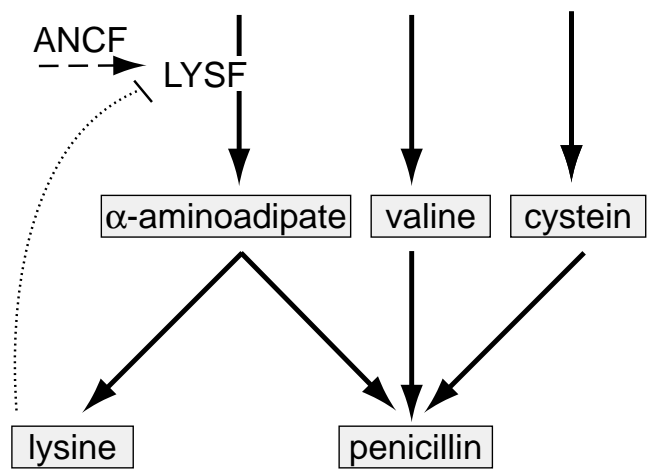

tryptophan acting as cross-pathway activator and tyrosine as feedback inhibitor (Fig. 1.7A) (Krappmann et al. 1999). (iii) Expression of LYSF, the homoaconitase acting in the common stem of lysine and penicillin biosynthesis, is regulated on at least two levels. Transcription of the lys $F$ gene is repressed by the HAP-like transcription factor AnCF and activated by an yet unidentified GATA-like transcription factor (Weidner et al. 2001). On protein level, LYSF is subject to end product repression by lysine (Weidner et al. 1997).

\subsubsection{Secondary metabolism}

1.3.2.1 Antibiotics and toxins. Secondary metabolites, whose biosynthesis relies on precursor molecules of primary metabolism, include the economically important microbial products like antibiotics and pigments. It is still obscure why secondary metabolites are produced at all, with hypotheses ranging from waste products to signal molecules (Firn and Jones 2000). Among the most prominent secondary metabolites are $\beta$-lactam antibiotics. Since penicillin is a block buster of pharmacological industry, much effort was directed into the study of mechanisms that regulate penicillin biosynthesis (Brakhage 1998). All three penicillin biosynthesis genes of $A$. nidulans, acvA, ipnA and aat $A$, have been cloned 
(MacCabe et al. 1990). The genes acvA and ipnA are divergently transcribed from a shared promoter region, whereas aat $A$ transcription is driven by a separate promoter. To date, no penicillin-specific regulators have been thoroughly characterised, but several regulators affect the synthesis of the antibiotic. This underscores the implication of regulatory networks that connect control of primary and secondary metabolism. The transcriptional activator AnCF (also known as PENR1) increases ipnA and aatA expression (Litzka et al. 1999). Penicillin production is subject to $\mathrm{pH}$ regulation by PACC being highest at alkaline $\mathrm{pH}$, whereas glucose repression of penicillin biosynthesis is mediated in a CREAindependent manner (Shah et al. 1991; Martin et al. 1999).

Another prominent secondary metabolite of $A$. nidulans is the polyketidederived mycotoxin sterigmatocystin, a precursor of aflatoxin. Its biosynthesis requires a cluster of at least 20 genes (Keller and Adams 1995). Regulation of penicillin biosynthesis seems to be connected to production of sterigmatocystin. The $\alpha$ subunit of a heterotrimeric G protein (FADA) acts negatively on sterigmatocystin and positive on penicillin syntheses (Tag et al. 2000). Moreover, sterigmatocystin synthesis is additionally linked to development (see 5.2.2.1).

1.3.2.2 Pigments and signal molecules. Beneath antibiotic and aflatoxin production, the fields of pigmentation and production of signal molecules are of major concern. A. nidulans produces pigments in various cell types, and again, amino acids are crucial precursor molecules. The brownish pigment melanin is formed from tyrosine by a phenoloxidase, a tyrosinase. It is partly secreted and partly accumulates as microfibrilles on the exterior of elder fungal hyphae (Martinelli et al. 1969; Pirt and Rowley 1969). Other pigments are restricted to the reproductive cycles (see 1.4.3 and 1.4.4), where they probably function to protect nucleic acids from damage by ultraviolet light. The conidiospore colour probably originates from tryptophan: 6-hydroxy- $\mathrm{N}$-acetyl-tryptophan is converted to a yellow pigment precursor and subsequently to the green pigment under contribution of a phenol oxidase, a polyketide synthase and a $p$-diphenol-oxidase, encoded by the genes ivo $B$ (Clutterbuck 1990), wA (Mayorga and Timberlake 1990) and $y A$ (Aramayo and Timberlake 1993), respectively. Expression of these gene products of conidiosporogenesis is mainly regulated by regulators specific for asexual development (see 1.4.3). The synthesis pathway of the red ascospore colour asperthecin is yet unknown. This anthraquinone is difficult to isolate because of its resistance to solvent extraction (Howard and Raistrick 1955). A phenol oxidase (laccase II) is specifically expressed during early sexual development in specialised structures (primordia and Hülle cells), but its contribution to pigment synthesis remains to be proven (Scherer and Fischer 1998).

Knowledge about biosynthesis of hormones, pheromones and other signal molecules in A. nidulans is even scarce. Two proposed signal molecules are probably linked to amino acid metabolism and one to fatty acid metabolism - and all three of them mediate developmental responses (see Chapter 4). During the course of this work, the tryptophan-related secondary metabolite auxin was identified as a probable contributor for differentiation processes (Eckert et al. 1999). 
Also, the diffusible extracellular ammonium-containing FluG factor is supposedly derived from amino acids, since its production depends on fluG that encodes a proposed glutamine synthetase activity (Lee and Adams 1994a; Yager et al. 1998). An endogenous mixture of hydroxylinoleic acid moieties, is known as Psi factor, precocious sexual inducer, a structural relative to vertebrate eicosanoid hormones. They comprise three interconvertible compounds, with the 5,8-dihydroxylinoleic acid PsiC as the most active compound that can be converted into its cyclic lactone PsiA. The 8-hydroxylinoleic acid PsiB is presumably an intermediate (Champe and el-Zayat 1989; Calvo et al. 1999; Calvo et al. 2001; Braus et al. 2002)

\subsection{Regulation of growth and development in A. nidulans}

A. nidulans is an ubiquitous saprophytic soil organism. A single colony is generally capable of hyphal growth as well as reproduction and can be regarded as a developmental unit. Growth and reproduction require sophisticated regulatory mechanisms, particularly in multicellular organisms that differentiate complex three dimensional structures (Fig. 1.8). Metabolite synthesis, nuclear distribution and cellular transport systems have to be remodelled, and processes as filamentous versus bud-like growth, cell cycle synchronisation or dis-synchronisation, mitosis versus meiosis and cellular differentiation must be initiated at the correct time and place. Genetic control of growth and development of $A$. nidulans is subject to intensive research. Several regulatory proteins (Tab. 1.2) and dependencies have been proposed, but their precise contribution to developmental decisions in A. nidulans remains to be elucidated.
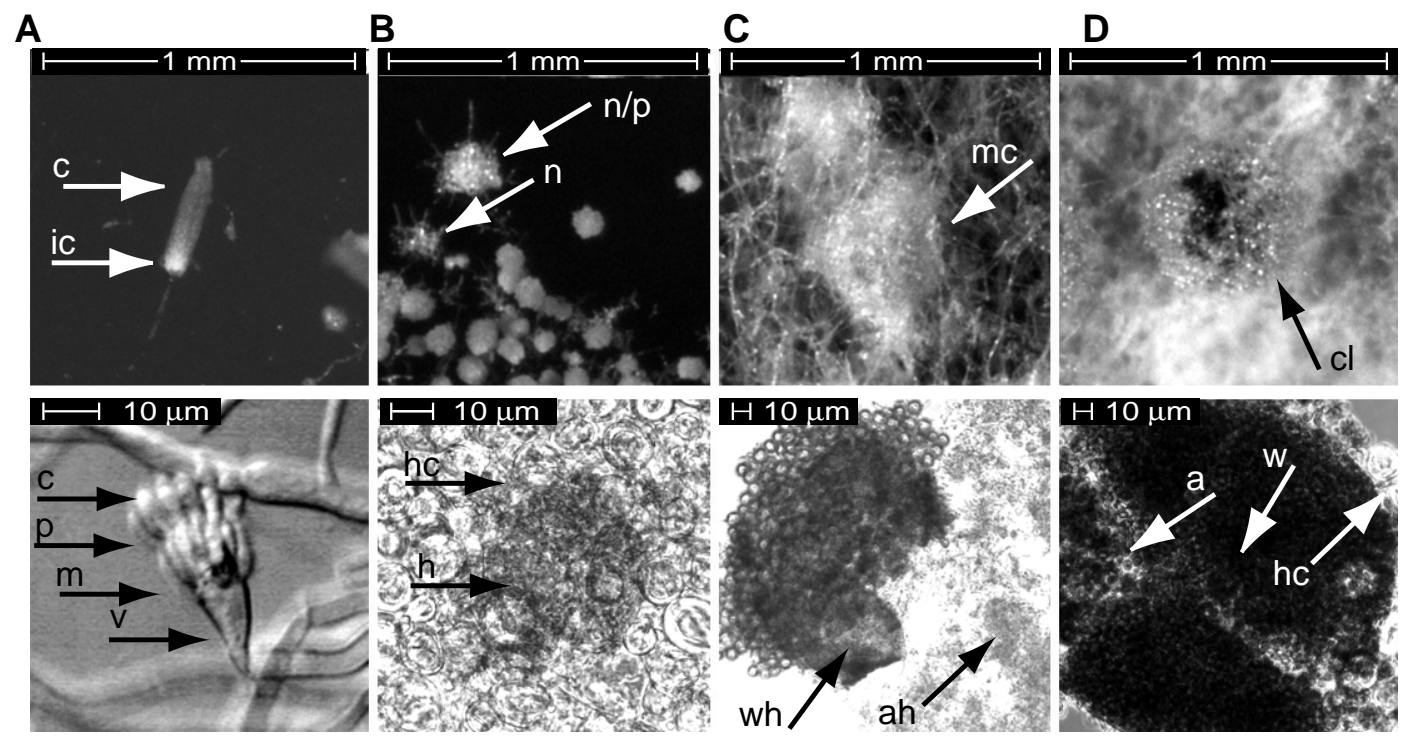

Fig. 1.8: Morphology of developmental structures of $\boldsymbol{A}$. nidulans. Pictures were taken under the binocular (upper row) and microscope (lower row). (A) Asexual development. Conidiophore architechture includes a vesicle $(\mathrm{v})$, the metulae $(\mathrm{m})$ and phialides $(\mathrm{p})$ and mature $(\mathrm{c})$ and immature (ic) conidiospores. (B) The first visible structure of sexual development are nest ( $n$ ) that contain the characteristic Huelle cells (hc). The primordium (p) looks makroscopically like a ball of hyphae (h). (C) In the mikro-cleistothecium, the dissection into inner ascogenous hphae (ah) and outer wall hyphae (wh) becomes obvious. (D) The mature cleistothecium (cl) is composed of a hard wall (w) and mature ascospores (a). 


\subsubsection{Germination and vegetative growth}

1.4.1.1 Morphology of growth. During vegetative growth, $A$. nidulans spreads over surfaces with filamentous, branched hyphae to occupy new nutritional sources. At germination, the G1-arrested asexual spores re-enter the cell cycle, swell by isotropic extension and switch to polarised hyphal growth to form the germ tube. In the growing hyphae, the apical cell accomplishes mitotic cycles resulting in an accumulation of nuclei. While the hyphal tip grows by apical extension, the cytoskeleton transports vesicles containing wall material and exoenzymes which fuse with the cell membrane to form the rigid chitineous cell wall and excrete exoenzymes. During growth, perforated septae composed of actin microfilaments isolate 3-4 nuclei in the subapical cells. These cells have a length of about $40 \mu \mathrm{m}$ and are arrested for growth and mitosis. They establish new polarity axes to form lateral branches with new apical cells under break-down of the cell wall at the future branch spot (McGoldrick et al. 1995; Momany et al. 1999).

1.4.1.2 Regulation of cellular organisation. On the molecular level, little is known about the onset of germination that requires co-ordination of growth, nuclear division and septation. Defects in vegetative hyphal growth mainly reflect general problems in cellular organisation processes like cell cycle control, cytoskeleton and polarity and thus often additionally effect the propagation cycles. Several components of these events have been identified by mutant analyses, while a complete picture of the regulatory connections remains to be established.

In eukaryotes, an important switch for perception of extracellular signals and subsequent transduction of this signal are heterotrimeric $G$ proteins composed of subunits $\alpha, \beta$ and $\gamma$, for example Ras proteins. Activated Ras proteins stimulate the adenylyl cyclase that converts ATP into the regulatory cAMP. This 'second messenger' in turn activates the protein kinase $A$ which modifies the activity of specific transcription factors by phosphorylation. For example, yeast Ras- and cAMP signalling is involved in the starvation-dependent control of budding versus pseudohyphal growth (Mösch 2000). An overall regulatory role in the integration of carbon source availability and the onset of germination in $A$. nidulans is proposed for RASA: initial high levels of RASA are essential for germination but subsequent gradual decrease of RASA activity is prerequisite for further development (Som and Kolaparthi 1994; Osherov and May 2000). This Ras signalling during germination is independent of cAMP signalling which also affects vegetative growth (Fillinger et al. 2002).

The cytoskeleton is characteristic for eukaryotic cells. Its dynamic structure of microfilaments and microtubules is important for the cellular scaffold and enables contractile functions. Moreover, transport of vesicles and cell organelles is dependent on this intercellular scaffold. Major components of this system are the structural tubulins and actins as well as motor proteins like dyneins and kinesins. Several factors that contribute to localisation events via the cytoskeleton have been identified in A. nidulans: nuclear distribution is affected in mutants of the nud (Ahn and Morris 2001) or tub genes (Kirk and Morris 1991; Kirk and Morris 1993) 
resulting in vegetative growth defects and aps $A, B$ mutants produce anucleate primary sterigmata (Fischer and Timberlake 1995; Sülmann et al. 1997). Strikingly, aps mutations are suppressed by the $\operatorname{samB}$ gene product that is involved in polarised growth (Krüger and Fischer 1998).

Polarity is an important feature of organisms that exhibit morphogenetic traits. A key feature of germination is the switch from isotropic swelling to polarised growth. Polarised growth requires the gene products of the pod, sep and hyp genes (Kaminskyj and Hamer 1998; Harris et al. 1999; Sharpless and Harris 2002), a mitogen-activated protein kinase MPKA (Bussink and Osmani 1999) and the protein phosphatase PPHA (Kosmidou et al. 2001).

Another characteristic of eukaryotic cells is the controlled proliferation program based on the cell cycle. Key components in the regulation of the cell cycle are cyclin-dependent protein kinases, highly conserved among eukaryotes, that control the transition between different cell cycle phases. Correspondingly, expression and degradation of the specific regulatory cyclin subunits are highly regulated. The growth or developmental mutant phenotype of several A. nidulans are founded on impaired cell cycle control and mitosis. Corresponding regulatory proteins have been identified, for example the "never in mitosis" kinases NIMA and NIMO (Lu et al. 1993; James et al. 1999) and the cyclin homologue PCLA (Schier et al. 2001; Schier and Fischer 2002). A striking effect resulting in binuclear conidiospores is seen in strains carrying the mutant bncA1 allele (Castiglioni Pascon et al. 2001).

\subsubsection{Developmental competence}

1.4.2.1 Environmental factors. About 16 to 20 hours after germination, elder subapical vegetative hyphae establish competence for further differentiation which implies an intrinsic time factor that controls developmental initiation (Axelrod et al. 1973). The knowledge about acquisition of developmental competence and subsequent induction of the reproductive cycles is scarce, but various physical, biochemical and genetic factors are involved (Fig. 1.9A). Only few pre-competence genes (aco) contributing to the establishment of competence have been identified in a genetic screen (Butnick et al. 1984a; Butnick et al. 1984b; Lewis and Champe 1995).

The two propagation cycles of $A$. nidulans probably have the potential to proceed independently, though only extreme conditions lead to formation of exclusively conidiophores or cleistothecia. As soon as competence is established, reproduction is induced strongly dependent on the environmental conditions. Nutrient availability plays an important role in developmental decisions. Either carbon or nitrogen starvation initiate asexual sporulation (Han et al. 1994; Skromne et al. 1995). It is suggested that carbon source availability activates a Rasdependent sensory mechanism with controlled rasA expression levels as mediator of ordered growth and development (Som and Kolaparthi 1994; Osherov and May 2000). RASA genetically interacts with the transcription factor DOPA, that in turn 
A

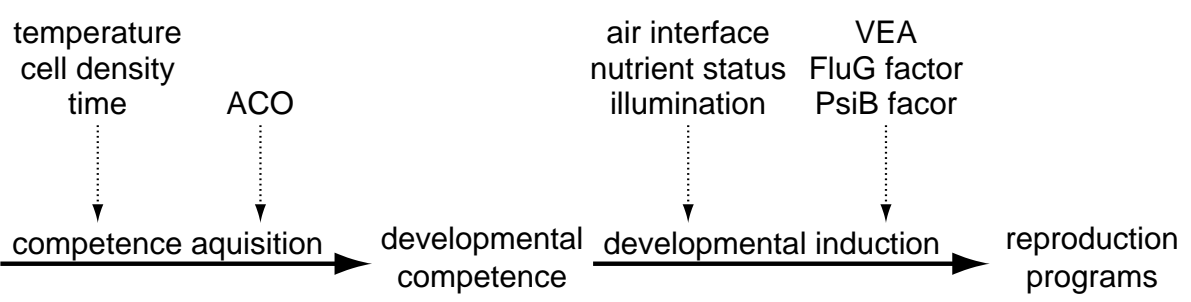

B

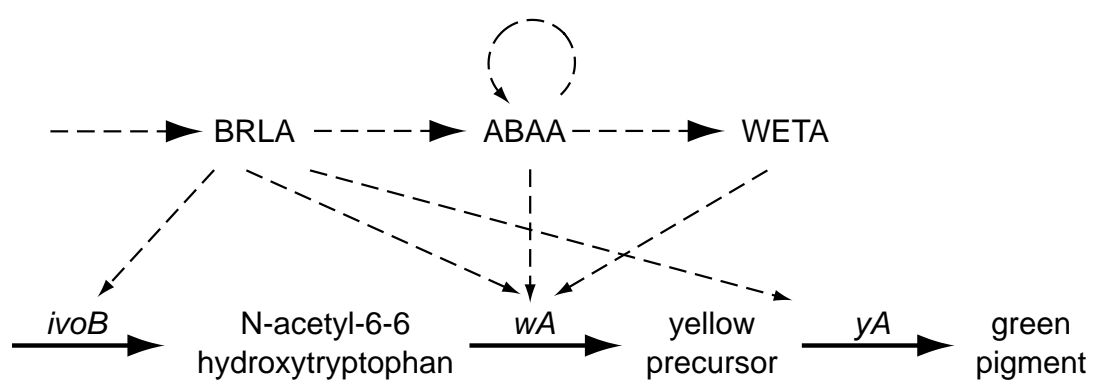

C

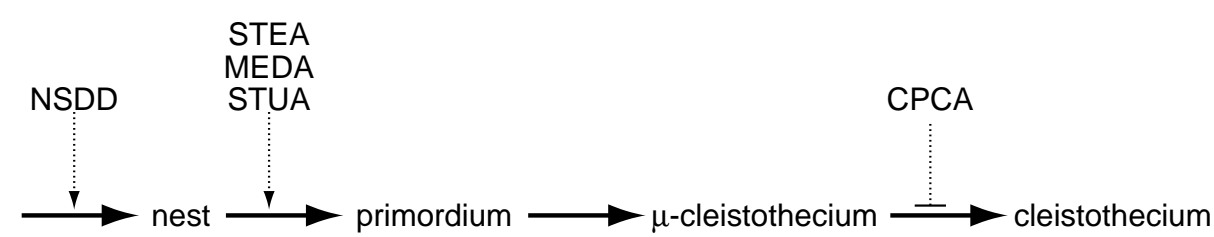

Fig. 1.9: Regulation of development in $\boldsymbol{A}$. nidulans. The schemes depict selected dependencies of regulatory proteins. Arrows show biochemical or morphological processes whereas broken arrow lines indicate a positive transcriptional regulation and dotted arrow lines a yet uncharacterised regulatory impact. (A) Competence aquisition and initiation of development. (B) Asexual development. The central cascade of transcription factors (BRLA, ABAA, WETA) controls several processes of conidiosporogenesis including the biosynthesis of the green conidiospore pigment by transcriptional activation of the corresponding genes. (C) Sexual development. The main morphological stages are shown with regulatory proteins whose level of action was proposed from mutant analyses.

mediates proper expression of several developmental transcriptions factors ( $b r / A$, $a b a A$ and steA; see Tab. 1.2) (Pascon and Miller 2000). Another important nutritional signal is the availability of phosphate. The putative cyclin dependent protein kinase PHOA, corresponding to the yeast Pho85 kinase, is involved in the developmental responses to phosphorus concentration, inoculation density and $\mathrm{pH}$ and probably integrates these environmental signals with developmental decisions in A. nidulans (Bussink and Osmani 1998).

Additionally, several physical factors are involved in the onset of differentiation processes. Prerequisite for development is a medium / air interface, though special growth conditions low in nitrogen exceptionally allow differentiation in submerged culture. Aeration directs the developmental program towards asexual reproduction, whereas high $\mathrm{CO}_{2}$ tension favours the sexual cycle (Axelrod et al. 1973). An important physical environmental factor that controls induction of development is illumination. Generally, A. nidulans produces predominantly conidiophores in the light and cleistothecia in the dark, though this light effect is 
dependent on medium composition and inoculation density (Raper and Fennell 1965; Zonneveld 1977; Mooney and Yager 1990). Moreover, the light-regulation of developmental decisions is dependent on the veA gene product. Most of the frequently used laboratory strains carry the mutant veA1 allele, causing lightindependent induction of asexual sporulation with concomitant increased levels of the asexual-specific transcription factor BRLA - and only rare induction of the sexual cycle. It is assumed that the truncated veA1 gene product mediates low functionality of the corresponding protein. VEA is thus hypothesised to repress initiation of asexual development and promotes the onset of sexual development (Mooney and Yager 1990).

1.4.2.2 Signal molecules. Two signal molecules involved in the initiation of developmental processes are the FluG and PsiC factors (see 1.3.2.2), and both seem to be linked to light response and the VEA protein. The FluG factor probably initiates asexual development at a certain threshold (Lee and Adams 1994a; Lee and Adams 1994b; Lee and Adams 1996). VEA was proposed to interact with a protein involved in the production of the FluG factor: FLUG (Yager et al. 1998). The fluG gene product in turn is dependent on the flbA gene product, a proposed regulator of $G$ protein signalling. FLBA probably controls the activity of a heterotrimeric $G$ protein ( $f a d A, s f a D)$ whose inactivation is essential for both asexual and sexual sporulation (Yu et al. 1996b; Rosen et al. 1999). Interestingly, $A$. nidulans flbA overproduction strains, as well as fluG and flbD overproducers, show increased expression of $b r l A$ and develop wild-type like conidiophores in submerged culture (Wieser and Adams 1995), emphasising their role in initiation of the asexual regulatory cascade. Mutants defective in these or the additional $f l b B$, $f l b C, f l b D$ and $f l b E$ loci show a characteristic phenotype of profuse aerial hyphae (Wieser and Adams 1995).

The PsiC factor acts as inducer of sexual development (Champe et al. 1987). Strikingly, the sexual inducing activity of the PsiC factor is abolished in veA1 mutant strains. A specific hormone receptor coupled to a signal transduction pathway is not yet identified for PsiC, but might be conceivable. The second hypothesis on PsiC function is its proposed activity in modification of membrane properties that might contribute to the fusion of specialised hyphae during sexual development. (Champe and el-Zayat 1989).

\subsubsection{Asexual reproduction}

1.4.3.1 Conidiophore morphology. Asexual reproduction of $A$. nidulans results in the differentiation of mitotically derived conidiospores about 48 hours after germination. Production of the asexual developmental unit, the conidiophore (Fig. 1.8A), can be microscopically observed as a linear cascade of morphological events as thoroughly reviewed (Adams et al. 1998). At first, the vegetative mycelium differentiates a thick-walled foot cell from which an aerial filament grows by vertical apical extension. This conidiophore stalk of about $100 \mu \mathrm{m}$ height and $4 \mu \mathrm{m}$ width swells at its tip to form a vesicle. Synchronised mitosis of nuclei and budding in a 
pseudohyphal-like appearance result in two rows of uninucleate sterigmata, the metulae and phialides. Each phialide mother successively buds at its tip with concurrent mitotic divisions, resulting in isogenic haploid nuclei encapsulated in a cell wall. The nucleus most distal to the phialide mother cell arrests at G1-phase and, during the following conidiosporogenesis, the cell wall gets impregnated and coloured characteristically green. Each phialide mother cell produces chains of up to 100 conidiospores that look like an aspergill used for catholic consecration inspiring the naming of the genus Aspergillus.

1.4.3.2 The asexual regulatory cascade. Specific regulation of asexual development is driven by a central regulatory cascade constituted of two major transcription factors: BRLA and ABAA (Fig. 1.9B). The brlA gene product is responsible for a developmental switch from apical growth to swelling of the vesicle and budding of the sterigmata (Miller et al. 1992). The brlA locus includes two overlapping transcription units, $b r l A \alpha$ and $b r l A \beta$, that both accumulate early during development and probably have redundant functions. Main effector is the brIA $\beta$ gene product that is expressed about 20 hours after germination and activates transcription of developmental genes like abaA, wet $A$ and $\operatorname{rod} A$ (Chang and Timberlake 1993; Han et al. 1993; Prade and Timberlake 1993). ABAA is required for the switch from sterigmata budding to formation of conidiospores. The abaA gene product is expressed about 24 hours after germination and activates transcription of several conidiation-specific genes including $w e t A, \operatorname{rot} A, y A, w A$.

The two-component protein kinase TCSA is essential for conidiation (Virginia et al. 2000), though it was not yet integrated into the regulatory cascade that drives the asexual circuit. Additionally, several regulators of conidiosporogenesis have been identified. The putative transcription factor WETA is essential for maturation of conidia including the inner wall layer and expression of the $w A$ gene (Marshall and Timberlake 1991), the rodA and $\operatorname{dew} A$ gene products contribute to formation of the outer hydrophobic protein layer (Stringer et al. 1991; Strauss et al. 1999) and the genes ivo $A$, ivoB, $w A$ and $y A$ are involved in the synthesis of the green polyketide-derived conidiospore pigment (see 1.3.2.2). Conidiosporogenesis is mainly driven by the regulatory backbone of conidiophore regulation (Fig. 1.9B) as seen for the transcriptional activation of the genes dedicated to pigment synthesis by BRLA and ABAA (Mayorga and Timberlake 1990; Aramayo and Timberlake 1993; Andrianopoulos and Timberlake 1994; Timberlake and Clutterbuck 1994).

\subsubsection{Sexual reproduction and developmental co-ordination}

1.4.4.1 Morphology of the cleistothecium. One of few representatives of the genus Aspergillus that are capable of meiotic reproduction is $A$. nidulans, in its sexual form also known as Emericella nidulans. Sexual propagation results in the differentiation of meiotically derived ascospores about 100 hours after germination. Development of the sexual reproduction unit, the cleistothecium, comprises several morphological process (Champe et al. 1994; Braus et al. 2002). About 50 hours 
after germination, the onset of sexual development is probably mediated by fusion of fertile hyphae. These are wrapped up with unordered mycelium of specialised vegetative hyphae that bud from the tip to produce Hülle cells. These globose cells contain tissue-specific proteins like a phenol oxidase (laccase II) and probably function as nurse cells. (Hermann et al. 1983; Scherer and Fischer 1998). The conglomerate of specialised hyphae and Hülle cells, the first visible structure of sexual development, is generally termed nest (Fig. 1.8B) - and was inspiring for the naming of the species as nest-former (nidulans). Within this nest, a cleistothecial primordium develops (Fig. 1.8B). After further differentiation to a structure termed micro-cleistothecium (Fig. 1.8C), a morphological bipartition into an outer sterile hyphal layer and an inner fertile cellular mass becomes visible under the microscope. The surrounding nest mycelium differentiates to form an outer network of flat and highly branched hyphae. The network is glued by the yet uncharacterised substance cleistin and finally matures to a compact surface, the cleistothecial wall (Champe et al. 1994). Within this developing shell, the dikaryotic hyphae undergo co-ordinated cellular and nuclear divisions. About 80 hours after germination, two nuclei are trapped in the topmost cell of the co-called crozier and fuse to form a zygote (Pontecorvo et al. 1953). From this diploid ascus mother cell, meiosis and two rounds of mitosis finally result in asci that contain eight nuclei. Each nucleus is encapsulated by a membrane and undergoes an additional mitotic division, resulting in binucleate ascospores. During ascosporogenesis, the membrane is differentiated into the ascospore wall by accumulation of a characteristic red pigment called asperthecin (see 1.3.2.2) and surface ornamentation. Mature cleistothecia (Fig. 1.8D) can reach a size of $200 \mu \mathrm{m}$ and enclose up to 80,000 ascospores (Champe et al. 1994).

1.4.4.2 Sexual tissue type-specific regulation. The precise timing of the events leading to a cleistothecium is not known to date. Also, knowledge about the regulatory circuits that drive sexual development in $A$. nidulans is rather fragmentary and is deduced mainly from the physiological defects of mutant strains (Fig. 1.9C). Two transcription factors, NSDD and STEA, are known to exclusively impact cleistothecia formation. $A$. nidulans $n s d D$ null mutants lack all sexual tissue types (Han et al. 2001) whereas steA deletion mutants are acleistothecial but still produce Hülle cells (Vallim et al. 2000). Other mutants with defects in the sexual cycle are less characterised. Several sexual sporulation mutants form externally normal cleistothecia but do not differentiate mature ascospores due to defects in crozier formation, karyogamy, meiosis or post-meiotic mitosis (Swart et al. 2001). Additionally, several mutants specifically abort the developmental process. For example, reduced carbon sources result in limited and blocked sexual development, probably because of absence of $\alpha-1,3$ glucan components for cleistothecial wall synthesis (Zonneveld 1975). Accordingly, strains lacking the Hülle cell-specific $\alpha-1,3$ glucanase mutA are acleistothecial (Wei et al. 2001). An effect of the cross-pathway activator CPC (Hoffmann et al. 2001) and auxin (Eckert et al. 1999) on sexual development was shown during the course of this work and will be discussed together with the results of this study in Chapter 5 . 
1.4.4.3 Developmental modifiers. Once developmental cycles are induced, several regulatory mechanisms seem to co-ordinate their molecular control. Two transcription factors have been termed as developmental modifiers of both pathways, STUA and MEDA. Transcript levels of stuA rise at competence, and increasing developmental stuA thresholds probably confer to vegetative growth, asexual propagation and sexual development (Wu and Miller 1997). A. nidulans stuA deletion strains develop disorganised asexual structures with conidiospores budding directly from the vesicle (Miller et al. 1991). Concerning sexual development, these mutants are acleistothecial but do produce Hülle cells (Dutton et al. 1997; Vallim et al. 2000). On the molecular level, STUA is required for the correct spatial distribution of BRLA and ABAA (Miller et al. 1992). Contrary, MEDA is responsible for proper temporal expression of $b r l A$ transcripts and also functions as co-activator of $a b a A$ expression (Busby et al. 1996). Strains with medA defects also show disorganised asexual structures, with branching metulae and secondary conidiophores. In regard to sexual development they are acleistothecial but do produce Hülle cells (Clutterbuck 1969). These developmental modifiers demonstrate that, beneath the complex regulation of specific asexual and sexual reproduction, a sophisticated cross-talk must exist between the two developmental pathways.

The physiological and morphological versatility of $A$. nidulans, as described in this chapter, implies interconnected control mechanisms that conduct the orchestra of molecular processes in the fungal organism. This work shows how regulatory networks that were originally described to control primary metabolism (crosspathway control) or development (COP9 signalosome-dependent network) additionally impact other circuits. Chapter 2 reveals a cross-connection between regulation of histidine biosynthesis by the cross-pathway control and sexual development of $A$. nidulans. Chapter 3 describes how the cross-pathway control contributes to the regulation of biosynthesis of the secondary metabolite penicillin. Last but not least, Chapter 4 reveals a link of the COP9 signalosome-dependent network to sexual development as well as production of a red pigment as secondary metabolite.

\section{$1.5 \quad$ References}

Abel, S., P.W. Oeller, and A. Theologis. 1994. Early auxin-induced genes encode short-lived nuclear proteins. Proc Natl Acad Sci U S A 91: 326-330.

Adams, T.H., J.K. Wieser, and J.H. Yu. 1998. Asexual sporulation in Aspergillus nidulans. Microbiol Mol Biol Rev 62: 35-54.

Ahn, C. and N.R. Morris. 2001. Nudf, a fungal homolog of the human LIS1 protein, functions as a dimer in vivo. J Biol Chem 276: 9903-9909.

Albrecht, G., H.-U. Mösch, B. Hoffmann, U. Reusser, and G.H. Braus. 1998. Monitoring the Gcn4 proteinmediated response in the yeast Saccharomyces cerevisiae. J Biol Chem 273: 12696-12702.

Alifano, P., R. Fani, P. Lio, A. Lazcano, M. Bazzicalupo, M.S. Carlomagno, and C.B. Bruni. 1996. Histidine biosynthetic pathway and genes: structure, regulation, and evolution. Microbiol Rev 60: 44-69.

Andrianopoulos, A. and M.J. Hynes. 1990. Sequence and functional analysis of the positively acting regulatory gene amdR from Aspergillus nidulans. Mol Cell Biol 10: 3194-3203. 
Andrianopoulos, A. and W.E. Timberlake. 1994. The Aspergillus nidulans abaA gene encodes a transcriptional activator that acts as a genetic switch to control development. Mol Cell Biol 14: 2503-2515.

Ang, L.H., S. Chattopadhyay, N. Wei, T. Oyama, K. Okada, A. Batschauer, and X.W. Deng. 1998. Molecular interaction between COP1 and HY5 defines a regulatory switch for light control of Arabidopsis development. Mol Cell 1: 213-222.

Aramayo, R. and W.E. Timberlake. 1993. The Aspergillus nidulans yA gene is regulated by abaA. EMBO J 12: 2039-2048.

Axelrod, D.E., M. Gealt, and M. Pastushok. 1973. Gene control of developmental competence in Aspergillus nidulans. Dev Biol 34: 9-15.

Bech-Otschir, D., R. Kraft, X. Huang, P. Henklein, B. Kapelari, C. Pollmann, and W. Dubiel. 2001. COP9 signalosome-specific phosphorylation targets p53 to degradation by the ubiquitin system. EMBO $J$ 20: $1630-1639$.

Bech-Otschir, D., M. Seeger, and W. Dubiel. 2002. The COP9 signalosome: at the interface between signal transduction and ubiquitin-dependent proteolysis. J Cell Sci 115: 467-473.

Bentley, R. 1990. The shikimate pathway--a metabolic tree with many branches. Crit Rev Biochem Mol Biol 25 : 307-384.

Bianchi, E., S. Denti, A. Granata, G. Bossi, J. Geginat, A. Villa, L. Rogge, and R. Pardi. 2000. Integrin LFA-1 interacts with the transcriptional co-activator JAB1 to modulate AP-1 activity. Nature 404: 617-621.

Bohmann, D., T.J. Bos, A. Admon, T. Nishimura, P.K. Vogt, and R. Tjian. 1987. Human proto-oncogene c-jun encodes a DNA binding protein with structural and functional properties of transcription factor AP-1. Science 238: 1386-1392.

Bounpheng, M.A., I.N. Melnikova, S.G. Dodds, H. Chen, N.G. Copeland, D.J. Gilbert, N.A. Jenkins, and B.A. Christy. 2000. Characterization of the mouse JAB1 cDNA and protein. Gene 242: 41-50.

Brakhage, A.A. 1998. Molecular regulation of $\beta$-lactam biosynthesis in filamentous fungi. Microbiol Mol Biol Rev 62: 547-585.

Braus, G.H., S. Krappmann, and S.E. Eckert. 2002. Sexual development in ascomycetes: fruit body formation of Aspergillus nidulans. In Molecular biology of fungal development (ed. H.D. Osiewacz), pp. 215244. Marcel Dekker, Inc., New York Basel.

Burger, G., J. Strauss, C. Scazzocchio, and B.F. Lang. 1991. nirA, the pathway-specific regulatory gene of nitrate assimilation in Aspergillus nidulans, encodes a putative GAL4-type zinc finger protein and contains four introns in highly conserved regions. Mol Cell Biol 11: 5746-5755.

Burley, S.K. and R.G. Roeder. 1996. Biochemistry and structural biology of transcription factor IID (TFIID). Annu Rev Biochem 65: 769-799.

Busby, T.M., K.Y. Miller, and B.L. Miller. 1996. Suppression and enhancement of the Aspergillus nidulans medusa mutation by altered dosage of the bristle and stunted genes. Genetics 143: 155-163.

Bussink, H.J. and S.A. Osmani. 1998. A cyclin-dependent kinase family member (PHOA) is required to link developmental fate to environmental conditions in Aspergillus nidulans. EMBO J 17: 3990-4003.

Bussink, H.J. and S.A. Osmani. 1999. A mitogen-activated protein kinase (MPKA) is involved in polarized growth in the filamentous fungus, Aspergillus nidulans. FEMS Microbiol Lett 173: 117-125.

Butnick, N.Z., L.N. Yager, T.E. Hermann, M.B. Kurtz, and S.P. Champe. 1984a. Mutants of Aspergillus nidulans blocked at an early stage of sporulation secrete an unusual metabolite. $J$ Bacteriol 160: 533-540.

Butnick, N.Z., L.N. Yager, M.B. Kurtz, and S.P. Champe. 1984b. Genetic analysis of mutants of Aspergillus nidulans blocked at an early stage of sporulation. J Bacteriol 160: 541-545.

Calvo, A.M., H.W. Gardner, and N.P. Keller. 2001. Genetic connection between fatty acid metabolism and sporulation in Aspergillus nidulans. J Biol Chem 276: 25766-25774.

Calvo, A.M., L.L. Hinze, H.W. Gardner, and N.P. Keller. 1999. Sporogenic effect of polyunsaturated fatty acids on development of Aspergillus spp. Appl Environ Microbiol 65: 3668-3673.

Castiglioni Pascon, R., A.A. Pizzirani-Kleiner, and B.L. Miller. 2001. The Aspergillus nidulans bncA1 mutation causes defects in the cell division cycle, nuclear movement and developmental morphogenesis. Mol Gen Genet 264: 546-554.

Chamovitz, D.A. and D. Segal. 2001. JAB1/CSN5 and the COP9 signalosome. A complex situation. EMBO Rep 2: 96-101.

Champe, S.P. and A.A. el-Zayat. 1989. Isolation of a sexual sporulation hormone from Aspergillus nidulans. $J$ Bacteriol 171: 3982-3988.

Champe, S.P., D.L. Nagle, and L.N. Yager. 1994. Sexual sporulation. In Aspergillus: 50 years on. Prog Ind Microbiol (ed. S.D. Martinelli and J.R. Klinghorn), pp. 429-54. Elsevier, Amsterdam.

Champe, S.P., P. Rao, and A. Chang. 1987. An endogenous inducer of sexual development in Aspergillus nidulans. J Gen Microbiol 133: 1383-1387.

Chang, Y.C. and W.E. Timberlake. 1993. Identification of Aspergillus brlA response elements (BREs) by genetic selection in yeast. Genetics 133: 29-38. 
Chauchereau, A., M. Georgiakaki, M. Perrin-Wolff, E. Milgrom, and H. Loosfelt. 2000. JAB1 interacts with both the progesterone receptor and SRC-1. J Biol Chem 275: 8540-8548.

Chi, Y., M.J. Huddleston, X. Zhang, R.A. Young, R.S. Annan, S.A. Carr, and R.J. Deshaies. 2001. Negative regulation of Gcn4 and Msn2 transcription factors by Srb10 cyclin-dependent kinase. Genes Dev 15: 1078-1092.

Claret, F.X., M. Hibi, S. Dhut, T. Toda, and M. Karin. 1996. A new group of conserved coactivators that increase the specificity of AP-1 transcription factors. Nature 383: 453-457.

Clutterbuck, A.J. 1969. A mutational analysis of conidial development in Aspergillus nidulans. Genetics 63: 317-327.

Clutterbuck, A.J. 1990. The genetics of conidiophore pigmentation in Aspergillus nidulans. J Gen Microbiol 136: $1731-1738$

Cohen, H., A. Azriel, T. Cohen, D. Meraro, S. Hashmueli, D. Bech-Otschir, R. Kraft, W. Dubiel, and B.Z. Levi. 2000. Interaction between interferon consensus sequence-binding protein and COP9/Signalosome subunit CSN2 (Trip15). J Biol Chem 275: 39081-39089.

Conlon, H., I. Zadra, H. Haas, H.N. Arst, Jr., M.G. Jones, and M.X. Caddick. 2001. The Aspergillus nidulans GATA transcription factor gene are $B$ encodes at least three proteins and features three classes of mutation. Mol Microbiol 40: 361-375.

Dechend, R., F. Hirano, K. Lehmann, V. Heissmeyer, S. Ansieau, F.G. Wulczyn, C. Scheidereit, and A. Leutz. 1999. The Bcl-3 oncoprotein acts as a bridging factor between NF-kappaB/Rel and nuclear coregulators. Oncogene 18: 3316-3323.

del Pozo, J.C., S. Dharmasiri, H. Hellmann, L. Walker, W.M. Gray, and M. Estelle. 2002. AXR1-ECR1dependent conjugation of RUB1 to the Arabidopsis Cullin AtCUL1 is required for auxin response. Plant Cell 14: 421-433.

Deng, X.W., W. Dubiel, N. Wei, K. Hofmann, K. Mundt, J. Colicelli, J. Kato, M. Naumann, D. Segal, M. Seeger, A. Carr, M. Glickman, and D.A. Chamovitz. 2000. Unified nomenclature for the COP9 signalosome and its subunits: an essential regulator of development. Trends Genet 16: 202-203.

Deshaies, R.J. 1999. SCF and Cullin/Ring H2-based ubiquitin ligases. Annu Rev Cell Dev Biol 15: 435-467.

Drysdale, C.M., E. Duenas, B.M. Jackson, U. Reusser, G.H. Braus, and A.G. Hinnebusch. 1995. The transcriptional activator GCN4 contains multiple activation domains that are critically dependent on hydrophobic amino acids. Mol Cell Biol 15: 1220-1233.

Drysdale, C.M., B.M. Jackson, R. McVeigh, E.R. Klebanow, Y. Bai, T. Kokubo, M. Swanson, Y. Nakatani, P.A. Weil, and A.G. Hinnebusch. 1998. The Gcn4p activation domain interacts specifically in vitro with RNA polymerase II holoenzyme, TFIID, and the Adap-Gcn5p coactivator complex. Mol Cell Biol 18: 1711-1724.

Dutton, J.R., S. Johns, and B.L. Miller. 1997. StuAp is a sequence-specific transcription factor that regulates developmental complexity in Aspergillus nidulans. EMBO J 16: 5710-5721.

Eckert, S.E. 2000. Tryptophan biosynthesis and sexual development of Aspergillus nidulans. In Dissertation, pp. 92-103, Friedrich-Alexander Universität Erlangen-Nürnberg.

Eckert, S.E., B. Hoffmann, C. Wanke, and G.H. Braus. 1999. Sexual development of Aspergillus nidulans in tryptophan auxotrophic strains. Arch Microbiol 172: 157-166.

Eckert, S.E., E. Kübler, B. Hoffmann, and G.H. Braus. 2000. The tryptophan synthase-encoding $\operatorname{trp} B$ gene of Aspergillus nidulans is regulated by the cross-pathway control system. Mol Gen Genet 263: 867-876.

Ellenberger, T.E., C.J. Brandl, K. Struhl, and S.C. Harrison. 1992. The GCN4 basic region leucine zipper binds DNA as a dimer of uninterrupted alpha helices: crystal structure of the protein-DNA complex. Cell 71: 1223-1237.

Engelberg, D., C. Klein, H. Martinetto, K. Struhl, and M. Karin. 1994. The UV response involving the Ras signaling pathway and AP-1 transcription factors is conserved between yeast and mammals. Cell 77: 381-390.

Espeso, E.A. and M.A. Penalva. 1996. Three binding sites for the Aspergillus nidulans PacC zinc-finger transcription factor are necessary and sufficient for regulation by ambient $\mathrm{pH}$ of the isopenicillin $\mathrm{N}$ synthase gene promoter. J Biol Chem 271: 28825-28830.

Espeso, E.A., J. Tilburn, L. Sanchez-Pulido, C.V. Brown, A. Valencia, H.N. Arst, Jr., and M.A. Penalva. 1997. Specific DNA recognition by the Aspergillus nidulans three zinc finger transcription factor PacC. J Mol Biol 274: 466-480.

Fan, M., X. Long, J.A. Bailey, C.A. Reed, E. Osborne, E.A. Gize, E.A. Kirk, R.M. Bigsby, and K.P. Nephew. 2002. The activating enzyme of NEDD8 inhibits steroid receptor function. Mol Endocrinol 16: 315330.

Farras, R., A. Ferrando, J. Jasik, T. Kleinow, L. Okresz, A. Tiburcio, K. Salchert, C. del Pozo, J. Schell, and C. Koncz. 2001. SKP1-SnRK protein kinase interactions mediate proteasomal binding of a plant SCF ubiquitin ligase. EMBO J 20: 2742-2756.

Felenbok, B., D. Sequeval, M. Mathieu, S. Sibley, D.I. Gwynne, and R.W. Davies. 1988. The ethanol regulon in Aspergillus nidulans: characterization and sequence of the positive regulatory gene alcR. Gene 73: 385-396. 
Fillinger, S., M.K. Chaveroche, K. Shimizu, N. Keller, and C. d'Enfert. 2002. cAMP and ras signalling independently control spore germination in the filamentous fungus Aspergillus nidulans. Mol Microbiol 44: 1001-1016.

Firn, R.D. and C.G. Jones. 2000. The evolution of secondary metabolism - a unifying model. Mol Microbiol 37: 989-994.

Fischer, R. and W.E. Timberlake. 1995. Aspergillus nidulans apsA (anucleate primary sterigmata) encodes a coiled-coil protein required for nuclear positioning and completion of asexual development. $J$ Cell Biol 128: 485-498.

Freilich, S., E. Oron, Y. Kapp, Y. Nevo-Caspi, S. Orgad, D. Segal, and D.A. Chamovitz. 1999. The COP9 signalosome is essential for development of Drosophila melanogaster. Curr Biol 9: 1187-1190.

Fu, H., N. Reis, Y. Lee, M.H. Glickman, and R.D. Vierstra. 2001. Subunit interaction maps for the regulatory particle of the 26S proteasome and the COP9 signalosome. EMBO $J$ 20: 7096-7107.

Glickman, M.H., D.M. Rubin, O. Coux, I. Wefes, G. Pfeifer, Z. Cjeka, W. Baumeister, V.A. Fried, and D. Finley. 1998. A subcomplex of the proteasome regulatory particle required for ubiquitin-conjugate degradation and related to the COP9-signalosome and elF3. Cell 94: 615-623.

Goc, A. and P. Weglenski. 1988. Regulatory region of the Aspergillus nidulans argB gene. Curr Genet 14: 425-429.

Goossens, A., T.E. Dever, A. Pascual-Ahuir, and R. Serrano. 2001. The protein kinase Gcn2p mediates sodium toxicity in yeast. $J$ Biol Chem 276: 30753-30760.

Grant, P.A., L. Duggan, J. Cote, S.M. Roberts, J.E. Brownell, R. Candau, R. Ohba, T. Owen-Hughes, C.D. Allis, F. Winston, S.L. Berger, and J.L. Workman. 1997. Yeast Gen5 functions in two multisubunit complexes to acetylate nucleosomal histones: characterization of an Ada complex and the SAGA (Spt/Ada) complex. Genes Dev 11: 1640-1650.

Gray, W.M. and I. Estelle. 2000. Function of the ubiquitin-proteasome pathway in auxin response. Trends Biochem Sci 25: 133-138.

Gray, W.M., S. Kepinski, D. Rouse, O. Leyser, and M. Estelle. 2001. Auxin regulates SCF(TIR1)-dependent degradation of AUX/IAA proteins. Nature 414: 271-276.

Green, M.R. 2000. TBP-associated factors (TAFIIs): multiple, selective transcriptional mediators in common complexes. Trends Biochem Sci 25: 59-63.

Grundmann, O., H.-U. Mösch, and G.H. Braus. 2001. Repression of GCN4 mRNA translation by nitrogen starvation in Saccharomyces cerevisiae. J Biol Chem 276: 25661-2571.

Haas, H., I. Zadra, G. Stoffler, and K. Angermayr. 1999. The Aspergillus nidulans GATA factor SREA is involved in regulation of siderophore biosynthesis and control of iron uptake. J Biol Chem 274: 46134619.

Han, D.M., Y.J. Han, K.S. Chae, K.Y. Jahng, and Y.H. Lee. 1994. Effects of various carbon sources on the development of Aspergillus nidulans with velA+ or velA1 allele. Korean. J. Mycol. 22: 332-337.

Han, K.H., K.Y. Han, J.H. Yu, K.S. Chae, K.Y. Jahng, and D.M. Han. 2001. The nsdD gene encodes a putative GATA-type transcription factor necessary for sexual development of Aspergillus nidulans. Mol Microbiol 41: 299-309.

Han, S., J. Navarro, R.A. Greve, and T.H. Adams. 1993. Translational repression of brlA expression prevents premature development in Aspergillus. EMBO Journal 12: 2449-2457.

Harris, S.D., A.F. Hofmann, H.W. Tedford, and M.P. Lee. 1999. Identification and characterization of genes required for hyphal morphogenesis in the filamentous fungus Aspergillus nidulans. Genetics 151: 1015-1025.

Hengartner, C.J., C.M. Thompson, J. Zhang, D.M. Chao, S.M. Liao, A.J. Koleske, S. Okamura, and R.A. Young. 1995. Association of an activator with an RNA polymerase II holoenzyme. Genes Dev 9: 897910.

Hermann, T.E., M.B. Kurtz, and S.P. Champe. 1983. Laccase localized in hulle cells and cleistothecial primordia of Aspergillus nidulans. J Bacteriol 154: 955-964.

Hershko, A. and A. Ciechanover. 1998. The ubiquitin system. Annu Rev Biochem 67: 425-479.

Hinnebusch, A.G. 1988. Mechanisms of gene regulation in the general control of amino acid biosynthesis in Saccharomyces cerevisiae. Microbiol Rev 52: 248-273.

Hinnebusch, A.G. 1997. Translational regulation of yeast GCN4. A window on factors that control initiatortRNA binding to the ribosome. J Biol Chem 272: 21661-21664.

Hinnebusch, A.G. and K. Natarajan. 2002. Gcn4p, a master regulator of gene expression, is controlled at multiple levels by diverse signals of starvation and stress. Eukaryotic Cell 1: 22-32.

Hoffmann, B., O. Valerius, M. Andermann, and G.H. Braus. 2001. Transcriptional autoregulation and inhibition of mRNA translation of amino acid regulator gene $c p c A$ of filamentous fungus Aspergillus nidulans. Mol Biol Cell 12: 2846-2857.

Hofmann, K. and P. Bucher. 1998. The PCl domain: a common theme in three multiprotein complexes. Trends Biochem Sci 23: 204-205. 
Hong, X., L. Xu, X. Li, Z. Zhai, and H. Shu. 2001. CSN3 interacts with IKK $\gamma$ and inhibits TNF- but not IL-1induced NF-K activation. FEBS Lett 499: 133-136.

Howard, B.H. and H. Raistrick. 1955. The colouring matters of species in the Aspergillus nidulans group. Biochem J 59: 476-484.

Jackson, B.M., C.M. Drysdale, K. Natarajan, and A.G. Hinnebusch. 1996. Identification of seven hydrophobic clusters in GCN4 making redundant contributions to transcriptional activation. Mol Cell Biol 16: 55575571.

Jackson, P.K. and A.G. Eldridge. 2002. The SCF ubiquitin ligase: an extended look. Mol Cell 9: 923-925.

James, S.W., K.A. Bullock, S.E. Gygax, B.A. Kraynack, R.A. Matura, J.A. MacLeod, K.K. McNeal, K.A. Prasauckas, P.C. Scacheri, H.L. Shenefiel, H.M. Tobin, and S.D. Wade. 1999. nimO, an Aspergillus gene related to budding yeast Dbf4, is required for DNA synthesis and mitotic checkpoint control. $J$ Cell Sci 112: 1313-1324.

Johnson, P.F. and S.L. McKnight. 1989. Eukaryotic transcriptional regulatory proteins. Annu Rev Biochem 58: 799-839.

Kaminskyj, S.G. and J.E. Hamer. 1998. hyp loci control cell pattern formation in the vegetative mycelium of Aspergillus nidulans. Genetics 148: 669-680.

Kapelari, B., D. Bech-Otschir, R. Hegerl, R. Schade, R. Dumdey, and W. Dubiel. 2000. Electron microscopy and subunit-subunit interaction studies reveal a first architecture of COP9 signalosome. $\mathrm{J} \mathrm{Mol} \mathrm{Biol}$ 300: 1169-1178.

Karin, M., Z. Liu, and E. Zandi. 1997. AP-1 function and regulation. Curr Opin Cell Biol 9: 240-6.

Kawakami, T., T. Chiba, T. Suzuki, K. Iwai, K. Yamanaka, N. Minato, H. Suzuki, N. Shimbara, Y. Hidaka, F. Osaka, M. Omata, and K. Tanaka. 2001. NEDD8 recruits E2-ubiquitin to SCF E3 ligase. EMBO J 20: 4003-4012.

Keller, N.P. and T.H. Adams. 1995. Analysis of a mycotoxin gene cluster in Aspergillus nidulans. SAAS Bull Biochem Biotechnol 8: 14-21.

Kim, T., K. Hofmann, A.G. von Arnim, and D.A. Chamovitz. 2001. PCl complexes: pretty complex interactions in diverse signaling pathways. Trends Plant Sci 6: 379-386.

Kim, Y.J., S. Bjorklund, Y. Li, M.H. Sayre, and R.D. Kornberg. 1994. A multiprotein mediator of transcriptional activation and its interaction with the C-terminal repeat domain of RNA polymerase II. Cell 77: 599608.

Kirk, K.E. and N.R. Morris. 1991. The tubB alpha-tubulin gene is essential for sexual development in Aspergillus nidulans. Genes Dev 5: 2014-2023.

Kirk, K.E. and N.R. Morris. 1993. Either $\alpha$-tubulin isogene product is sufficient for microtubule function during all stages of growth and differentiation in Aspergillus nidulans. Mol Cell Biol 13: 4465-4476.

Kleemann, R., A. Hausser, G. Geiger, R. Mischke, A. Burger-Kentischer, O. Flieger, F.J. Johannes, T. Roger, T. Calandra, A. Kapurniotu, M. Grell, D. Finkelmeier, H. Brunner, and J. Bernhagen. 2000. Intracellular action of the cytokine MIF to modulate AP-1 activity and the cell cycle through Jab1. Nature 408: 211-216.

Klevit, R.E. 1991. Recognition of DNA by Cys2,His2 zinc fingers. Science 253: 1367-1393.

Kontoyiannis, D.P. and G.P. Bodey. 2002. Invasive aspergillosis in 2002: an update. Eur J Clin Microbiol Infect Dis 21: 161-172.

Kornitzer, D., B. Raboy, R.G. Kulka, and G.R. Fink. 1994. Regulated degradation of the transcription factor Gcn4. EMBO J 13: 6021-6030.

Kosmidou, E., P. Lunness, and J.H. Doonan. 2001. A type 2A protein phosphatase gene from Aspergillus nidulans is involved in hyphal morphogenesis. Curr Genet 39: 25-34.

Krappmann, S., K. Helmstaedt, T. Gerstberger, S. Eckert, B. Hoffmann, M. Hoppert, G. Schnappauf, and G.H. Braus. 1999. The aroC gene of Aspergillus nidulans codes for a monofunctional, allosterically regulated chorismate mutase. J Biol Chem 274: 22275-22282.

Krüger, M. and R. Fischer. 1998. Integrity of a Zn finger-like domain in SamB is crucial for morphogenesis in ascomycetous fungi. EMBO $\mathrm{J}$ 17: 204-214.

Kwok, S.F., J.M. Staub, and X.W. Deng. 1999. Characterization of two subunits of Arabidopsis 19S proteasome regulatory complex and its possible interaction with the COP9 complex. $\mathrm{J} \mathrm{Mol} \mathrm{Biol} \mathrm{285:}$ 85-95.

Lee, B.N. and T.H. Adams. 1994a. The Aspergillus nidulans fluG gene is required for production of an extracellular developmental signal and is related to prokaryotic glutamine synthetase I. Genes Dev 8: 641-651.

Lee, B.N. and T.H. Adams. 1994b. Overexpression of flbA, an early regulator of Aspergillus asexual sporulation, leads to activation of brlA and premature initiation of development. Mol Microbiol 14: 323334.

Lee, B.N. and T.H. Adams. 1996. FluG and flbA function interdependently to initiate conidiophore development in Aspergillus nidulans through brlA beta activation. EMBO J 15: 299-309. 
Lee, J.W., H.s. Choi, J. Gyuris, R. Brent, and D.D. Moore. 1995. Teo classes of proteins dependent on either the presence or absence of thyroid hormone for interaction with the thyroid hormone receptor. Mol. Endocrinol. 9: 243-254.

Lewis, C. and S.P. Champe. 1995. A pre-induction sporulation gene from Aspergillus nidulans. Microbiology 141: $1821-1828$.

$\mathrm{Li}$, S., X. Liu, and M. Ascoli. 2000. p38JAB1 binds to the intracellular precursor of the lutropin/choriogonadotropin receptor and promotes its degradation. J Biol Chem 275: 13386-13393.

Litzka, O., K. Then Bergh, J. Van den Brulle, S. Steidl, and A.A. Brakhage. 1999. Transcriptional control of expression of fungal beta-lactam biosynthesis genes. Antonie Van Leeuwenhoek 75: 95-105.

Lu, K.P., S.A. Osmani, and A.R. Means. 1993. Properties and regulation of the cell cycle-specific NIMA protein kinase of Aspergillus nidulans. J Biol Chem 268: 8769-8776.

Lyapina, S., G. Cope, A. Shevchenko, G. Serino, T. Tsuge, C. Zhou, D.A. Wolf, N. Wei, and R.J. Deshaies. 2001. Promotion of NEDD-CUL1 conjugate cleavage by COP9 signalosome. Science 292: 1382-1385.

MacCabe, A.P., M.B. Riach, S.E. Unkles, and J.R. Kinghorn. 1990. The Aspergillus nidulans npeA locus consists of three contiguous genes required for penicillin biosynthesis. EMBO J 9: 279-287.

Mahalingam, S., V. Ayyavoo, M. Patel, T. Kieber-Emmons, G.D. Kao, R.J. Muschel, and D.B. Weiner. 1998. HIV-1 Vpr interacts with a human 34-kDa mov34 homologue, a cellular factor linked to the G2/M phase transition of the mammalian cell cycle. Proc Natl Acad Sci U S A 95: 3419-3424.

Marshall, M.A. and W.E. Timberlake. 1991. Aspergillus nidulans wetA activates spore-specific gene expression. Mol Cell Biol 11: 55-62.

Martin, J.F., J. Casqueiro, K. Kosalkova, A.T. Marcos, and S. Gutierrez. 1999. Penicillin and cephalosporin biosynthesis: mechanism of carbon catabolite regulation of penicillin production. Antonie Van Leeuwenhoek 75: 21-31.

Martinelli, S.D., B.W. Bainbridge, and S.J. Pirt. 1969. Phenol oxidases produced by mutant and wild-type strains of Aspergillus nidulans. Biochem J 114: 10P.

Mayorga, M.E. and W.E. Timberlake. 1990. Isolation and molecular characterization of the Aspergillus nidulans wA gene. Genetics 126: 73-79.

McGoldrick, C.A., C. Gruver, and G.S. May. 1995. myoA of Aspergillus nidulans encodes an essential myosin I required for secretion and polarized growth. J Cell Biol 128: 577-587.

Meimoun, A., T. Holtzman, Z. Weissman, H.J. McBride, D.J. Stillman, G.R. Fink, and D. Kornitzer. 2000. Degradation of the transcription factor Gcn4 requires the kinase Pho85 and the SCF(CDC4) ubiquitinligase complex. Mol Biol Cell 11: 915-927.

Miller, K.Y., T.M. Toennis, T.H. Adams, and B.L. Miller. 1991. Isolation and transcriptional characterization of a morphological modifier: the Aspergillus nidulans stunted (stuA) gene. Mol Gen Genet 227: 285-292.

Miller, K.Y., J. Wu, and B.L. Miller. 1992. StuA is required for cell pattern formation in Aspergillus. Genes Dev 6: $1770-1782$.

Momany, M., P.J. Westfall, and G. Abramowsky. 1999. Aspergillus nidulans swo mutants show defects in polarity establishment, polarity maintenance and hyphal morphogenesis. Genetics 151: 557-567.

Mooney, J.L. and L.N. Yager. 1990. Light is required for conidiation in Aspergillus nidulans. Genes Dev 4: 1473-1482.

Morimoto, M., T. Nishida, R. Honda, and H. Yasuda. 2000. Modification of cullin-1 by ubiquitin-like protein Nedd8 enhances the activity of SCF(skp2) toward p27(kip1). Biochem Biophys Res Commun 270: 1093-1096.

Mösch, H.U. 2000. Pseudohyphal development of Saccharomyces cerevisiae. In Dimorphism in human paathogenic and apathogenis yeasts (ed. J.F. Ernst and A. Schmidt), pp. 185-200. Karger, Basel.

Mundt, K.E., C. Liu, and A.M. Carr. 2002. Deletion mutants in COP9/signalosome subunits in fission yeast Schizosaccharomyces pombe display distinct phenotypes. Mol Biol Cell 13: 493-502.

Mundt, K.E., J. Porte, J.M. Murray, C. Brikos, P.U. Christensen, T. Caspari, I.M. Hagan, J.B. Millar, V. Simanis, K. Hofmann, and A.M. Carr. 1999. The COP9/signalosome complex is conserved in fission yeast and has a role in S phase. Curr Biol 9: 1427-1430.

Musti, A.M., M. Treier, and D. Bohmann. 1997. Reduced ubiquitin-dependent degradation of c-Jun after phosphorylation by MAP kinases. Science 275: 400-402.

Natarajan, K., B.M. Jackson, H. Zhou, F. Winston, and A.G. Hinnebusch. 1999. Transcriptional activation by Gcn $4 p$ involves independent interactions with the SWI/SNF complex and the SRB/mediator. Mol Cell 4: 657-664.

Natarajan, K., M.R. Meyer, B.M. Jackson, D. Slade, C. Roberts, A.G. Hinnebusch, and M.J. Marton. 2001. Transcriptional profiling shows that Gcn4p is a master regulator of gene expression during amino acid starvation in yeast. Mol Cell Biol 21: 4347-4368.

Naumann, M., D. Bech-Otschir, X. Huang, K. Ferrell, and W. Dubiel. 1999. COP9 signalosome-directed C-Jun activation/stabilization is independent of JNK. J Biol Chem 274: 35297-35300. 
Nemeth, K., K. Salchert, P. Putnoky, R. Bhalerao, Z. Koncz-Kalman, B. Stankovic-Stangeland, L. Bako, J. Mathur, L. Okresz, S. Stabel, P. Geigenberger, M. Stitt, G.P. Redei, J. Schell, and C. Koncz. 1998. Pleiotropic control of glucose and hormone responses by PRL1, a nuclear WD protein, in Arabidopsis. Genes Dev 12: 3059-3073.

Noventa-Jordao, M.A., A.M. do Nascimento, M.H. Goldman, H.F. Terenzi, and G.H. Goldman. 2000. Molecular characterization of ubiquitin genes from Aspergillus nidulans: mRNA expression on different stress and growth conditions. Biochim Biophys Acta 1490: 237-244.

Ohh, M., W.Y. Kim, J.J. Moslehi, Y. Chen, V. Chau, M.A. Read, and W.G. Kaelin, Jr. 2002. An intact NEDD8 pathway is required for cullin-dependent ubiquitylation in mammalian cells. EMBO Rep 3: 177-182.

Oliphant, A.R., C.J. Brandl, and K. Struhl. 1989. Defining the sequence specificity of DNA-binding proteins by selecting binding sites from random-sequence oligonucleotides: analysis of yeast GCN4 protein. $\mathrm{Mol}$ Cell Biol 9: 2944-2949.

Osherov, N. and G. May. 2000. Conidial germination in Aspergillus nidulans requires RAS signaling and protein synthesis. Genetics 155: 647-656.

Osterlund, M.T., L.H. Ang, and X.W. Deng. 1999. The role of COP1 in repression of Arabidopsis photomorphogenic development. Trends Cell Biol 9: 113-118.

Osterlund, M.T., C.S. Hardtke, N. Wei, and X.W. Deng. 2000. Targeted destabilization of HY5 during lightregulated development of Arabidopsis. Nature 405: 462-466.

Paluh, J.L., M.J. Orbach, T.L. Legerton, and C. Yanofsky. 1988. The cross-pathway control gene of Neurospora crassa, $c p c-1$, encodes a protein similar to GCN4 of yeast and the DNA-binding domain of the oncogene v-jun-encoded protein. Proc Natl Acad Sci U S A 85: 3728-3732.

Panozzo, C., E. Cornillot, and B. Felenbok. 1998. The CreA repressor is the sole DNA-binding protein responsible for carbon catabolite repression of the alcA gene in Aspergillus nidulans via its binding to a couple of specific sites. J Biol Chem 273: 6367-6372.

Pascon, R.C. and B.L. Miller. 2000. Morphogenesis in Aspergillus nidulans requires Dopey (DopA), a member of a novel family of leucine zipper-like proteins conserved from yeast to humans. Mol Microbiol 36: 1250-1264.

Peng, Z., G. Serino, and X.W. Deng. 2001a. Molecular characterization of subunit 6 of the COP9 signalosome and its role in multifaceted developmental processes in Arabidopsis. Plant Cell 13: 2393-23407.

Peng, Z., G. Serino, and X.W. Deng. 2001b. A role of Arabidopsis COP9 signalosome in multifaceted developmental processes revealed by the characterization of its subunit 3. Development 128: 42774288.

Piotrowska, M. 1980. Cross-pathway regulation of ornithine carbamoyltransferase synthesis in Aspergillus nidulans. J Gen Microbiol 116: 335-339.

Pirt, S.J. and B.I. Rowley. 1969. Melanin production in Aspergillus nidulans. Biochem J 114: 9P-10P.

Platt, A., T. Langdon, H.N. Arst, Jr., D. Kirk, D. Tollervey, J.M. Sanchez, and M.X. Caddick. 1996. Nitrogen metabolite signalling involves the C-terminus and the GATA domain of the Aspergillus transcription factor AREA and the 3' untranslated region of its mRNA. EMBO J 15: 2791-2801.

Podust, V.N., J.E. Brownell, T.B. Gladysheva, R.S. Luo, C. Wang, M.B. Coggins, J.W. Pierce, E.S. Lightcap, and V. Chau. 2000. A Nedd8 conjugation pathway is essential for proteolytic targeting of p27Kip1 by ubiquitination. Proc Natl Acad Sci U S A 97: 4579-4584.

Pollmann, C., X. Huang, J. Mall, D. Bech-Otschir, M. Naumann, and W. Dubiel. 2001. The constitutive photomorphogenesis 9 signalosome directs vascular endothelial growth factor production in tumor cells. Cancer Res 61: 8416-8421.

Pontecorvo, G., J.A. Roper, L.M. Hemmons, K.D. MacDonald, and A.W.J. Bufton. 1953. The Genetics of Aspergillus nidulans. Advances in Genetics 5: 141-239.

Prade, R.A. and W.E. Timberlake. 1993. The Aspergillus nidulans brlA regulatory locus consists of overlapping transcription units that are individually required for conidiophore development. EMBO $J$ 12: $2439-2447$.

Pries, R., K. Bömeke, O. Grundmann, and G.H. Braus. 2002. Amino acid-dependent Gcn4p stability regulation occurs exclusively in the yeast nucleus. Euk Cell 1: in process citation.

Pu, W.T. and K. Struhl. 1991. The leucine zipper symmetrically positions the adjacent basic regions for specific DNA binding. Proc Natl Acad Sci U S A 88: 6901-6905.

Punt, P.J., J. Strauss, R. Smit, J.R. Kinghorn, C.A. van den Hondel, and C. Scazzocchio. 1995. The intergenic region between the divergently transcribed niiA and niaD genes of Aspergillus nidulans contains multiple NirA binding sites which act bidirectionally. Mol Cell Biol 15: 5688-5699.

Qiu, H., J. Dong, C. Hu, C.S. Francklyn, and A.G. Hinnebusch. 2001. The tRNA-binding moiety in GCN2 contains a dimerization domain that interacts with the kinase domain and is required for tRNA binding and kinase activation. EMBO $\mathrm{J}$ 20: 1425-1438.

Raper, K.B. and D.I. Fennell. 1965. The Genus Aspergillus. Williams and Wilkins, Baltimore. 
Read, M.A., J.E. Brownell, T.B. Gladysheva, M. Hottelet, L.A. Parent, M.B. Coggins, J.W. Pierce, V.N. Podust, R.S. Luo, V. Chau, and V.J. Palombella. 2000. Nedd8 modification of cul-1 activates SCF $\beta(\operatorname{TrCP})$-dependent ubiquitination of I $\mathrm{BB} \alpha$. Mol Cell Biol 20: 2326-2333.

Rolfes, R.J. and A.G. Hinnebusch. 1993. Translation of the yeast transcriptional activator GCN4 is stimulated by purine limitation: implications for activation of the protein kinase GCN2. Mol Cell Biol 13: 50995111.

Rosen, S., J.H. Yu, and T.H. Adams. 1999. The Aspergillus nidulans sfaD gene encodes a G protein $\beta$ subunit that is required for normal growth and repression of sporulation. EMBO J 18: 5592-5600.

Saudek, V., A. Pastore, M.A. Castiglione Morelli, R. Frank, H. Gausepohl, T. Gibson, F. Weih, and P. Roesch. 1990. Solution structure of the DNA-binding domain of the yeast transcriptional activator protein GCN4. Protein Eng 4: 3-10.

Scherer, M. and R. Fischer. 1998. Purification and characterization of laccase II of Aspergillus nidulans. Arch Microbiol 170: 78-84.

Schier, N. and R. Fischer. 2002. The Aspergillus nidulans cyclin PclA accumulates in the nucleus and interacts with the central cell cycle regulator NimX(Cdc2). FEBS Lett 523: 143-16.

Schier, N., R. Liese, and R. Fischer. 2001. A Pcl-Like Cyclin of Aspergillus nidulans Is Transcriptionally Activated by Developmental Regulators and Is Involved in Sporulation. Mol Cell Biol 21: 4075-4088.

Schwechheimer, C. and X. Deng. 2001. COP9 signalosome revisited: a novel mediator of protein degradation. Trends Cell Biol 11: 420-426.

Schwechheimer, C. and X.W. Deng. 2000. The COP/DET/FUS proteins-regulators of eukaryotic growth and development. Semin Cell Dev Biol 11: 495-503.

Schwechheimer, C., G. Serino, J. Callis, W.L. Crosby, S. Lyapina, R.J. Deshaies, W.M. Gray, M. Estelle, and X.W. Deng. 2001. Interactions of the COP9 signalosome with the E3 ubiquitin ligase SCFTIRI in mediating auxin response. Science 292: 1379-1382.

Seeger, M., C. Gordon, and W. Dubiel. 2001. Protein stability: The COP9 signalosome gets in on the act. Curr Biol 11: R643-6.

Seeger, M., R. Kraft, K. Ferrell, D. Bech-Otschir, R. Dumdey, R. Schade, C. Gordon, M. Naumann, and W. Dubiel. 1998. A novel protein complex involved in signal transduction possessing similarities to $26 \mathrm{~S}$ proteasome subunits. Faseb J 12: 469-478.

Shah, A.J., J. Tilburn, M.W. Adlard, and H.N. Arst, Jr. 1991. pH regulation of penicillin production in Aspergillus nidulans. FEMS Microbiol Lett 61: 209-212.

Sharpless, K.E. and S.D. Harris. 2002. Functional characterization and localization of the Aspergillus nidulans formin SEPA. Mol Biol Cell 13: 469-479.

Skromne, I., O. Sanchez, and J. Aguirre. 1995. Starvation stress modulates the expression of the Aspergillus nidulans brlA regulatory gene. Microbiology 141: 21-28.

Som, T. and V.S. Kolaparthi. 1994. Developmental decisions in Aspergillus nidulans are modulated by Ras activity. Mol Cell Biol 14: 5333-5348.

Steidl, S., P. Papagiannopoulos, O. Litzka, A. Andrianopoulos, M.A. Davis, A.A. Brakhage, and M.J. Hynes. 1999. AnCF, the CCAAT binding complex of Aspergillus nidulans, contains products of the hapB, $h a p C$, and hapE genes and is required for activation by the pathway-specific regulatory gene amdR. Mol Cell Biol 19: 99-106.

Strauss, J., H.K. Horvath, B.M. Abdallah, J. Kindermann, R.L. Mach, and C.P. Kubicek. 1999. The function of $\mathrm{CreA}$, the carbon catabolite repressor of Aspergillus nidulans, is regulated at the transcriptional and post-transcriptional level. Mol Microbiol 32: 169-178.

Stringer, M.A., R.A. Dean, T.C. Sewall, and W.E. Timberlake. 1991. Rodletless, a new Aspergillus developmental mutant induced by directed gene inactivation. Genes Dev 5: 1161-1171.

Strittmatter, A.W., S. Irniger, and G.H. Braus. 2001. Induction of jlbA mRNA synthesis for a putative bZIP protein of Aspergillus nidulans by amino acid starvation. Curr Genet 39: 327-334.

Suarez, T., M.V. de Queiroz, N. Oestreicher, and C. Scazzocchio. 1995. The sequence and binding specificity of UaY, the specific regulator of the purine utilization pathway in Aspergillus nidulans, suggest an evolutionary relationship with the PPR1 protein of Saccharomyces cerevisiae. EMBO $J$ 14: $1453-1467$.

Sülmann, R., N. Sievers, and R. Fischer. 1997. Nuclear traffic in fungal hyphae: in vivo study of nuclear migration and positioning in Aspergillus nidulans. Mol Microbiol 25: 757-769.

Suzuki, G., Y. Yanagawa, S.F. Kwok, M. Matsui, and X.W. Deng. 2002. Arabidopsis COP10 is a ubiquitinconjugating enzyme variant that acts together with COP1 and the COP9 signalosome in repressing photomorphogenesis. Genes Dev 16: 554-559.

Swart, K., D. van Heemst, M. Slakhorst, F. Debets, and C. Heyting. 2001. Isolation and characterization of sexual sporulation mutants of Aspergillus nidulans. Fungal Genet Biol 33: 25-35.

Tag, A., J. Hicks, G. Garifullina, C. Ake, Jr., T.D. Phillips, M. Beremand, and N. Keller. 2000. G-protein signalling mediates differential production of toxic secondary metabolites. Mol Microbiol 38: 658-665. 
Takemaru, K., S. Harashima, H. Ueda, and S. Hirose. 1998. Yeast coactivator MBF1 mediates GCN4dependent transcriptional activation. Mol Cell Biol 18: 4971-4976.

Tansey, W.P. 2001. Transcriptional activation: risky business. Genes Dev 15: 1045-1050.

Timberlake, W.E. and A.J. Clutterbuck. 1994. Genetic regulation of conidiation. In Aspergillus: 50 years on (ed. S.D. Martinelli and J.R. Klinghorn), pp. 1-851. Elsevier, Amsterdam.

Tomoda, K., Y. Kubota, and J. Kato. 1999. Degradation of the cyclin-dependent-kinase inhibitor p27Kip1 is instigated by Jab1. Nature 398: 160-165.

Tongaonkar, P., L. Chen, D. Lambertson, B. Ko, and K. Madura. 2000. Evidence for an interaction between ubiquitin-conjugating enzymes and the 26S proteasome. Mol Cell Biol 20: 4691-4698.

Tsuge, T., M. Matsui, and N. Wei. 2001. The subunit 1 of the COP9 signalosome suppresses gene expression through its $\mathrm{N}$-terminal domain and incorporates into the complex through the PCl domain. $\mathrm{J} \mathrm{Mol} \mathrm{Biol}$ 305: 1-9.

Tyers, M. and P. Jorgensen. 2000. Proteolysis and the cell cycle: with this RING I do thee destroy. Curr Opin Genet Dev 10: 54-64.

Valerius, O., O. Draht, E. Kübler, K. Adler, B. Hoffmann, and G.H. Braus. 2001. Regulation of hisHF transcription of Aspergillus nidulans by adenine and amino acid limitation. Fungal Genet Biol 32: 2131.

Vallim, M.A., K.Y. Miller, and B.L. Miller. 2000. Aspergillus SteA (sterile12-like) is a homeodomain-C2/H2-Zn+2 finger transcription factor required for sexual reproduction. Mol Microbiol 36: 290-301.

Verma, R., S. Chen, R. Feldman, D. Schieltz, J. Yates, J. Dohmen, and R.J. Deshaies. 2000. Proteasomal proteomics: identification of nucleotide-sensitive proteasome-interacting proteins by mass spectrometric analysis of affinity-purified proteasomes. Mol Biol Cell 11: 3425-3439.

Virginia, M., C.L. Appleyard, W.L. McPheat, and M.J. Stark. 2000. A novel 'two-component' protein containing histidine kinase and response regulator domains required for sporulation in Aspergillus nidulans. Curr Genet 37: 364-372.

von Arnim, A.G. 2001. A hitchhiker's guide to the proteasome. Sci STKE 2001: E2.

Wang, X., D. Kang, S. Feng, G. Serino, C. Schwechheimer, and N. Wei. 2002. CSN1 N-terminal-dependent activity is required for Arabidopsis development but not for Rub1/Nedd8 deconjugation of cullins: a structure-function study of CSN1 subunit of COP9 signalosome. Mol Biol Cell 13: 646-655.

Wanke, C., S. Eckert, G. Albrecht, W. van Hartingsveldt, P.J. Punt, C.A. van den Hondel, and G.H. Braus. 1997. The Aspergillus niger GCN4 homologue, $c p c A$, is transcriptionally regulated and encodes an unusual leucine zipper. Mol Microbiol 23: 23-33.

Watson, J.D. and F.H. Crick. 1974. Molecular structure of nucleic acids: a structure for deoxyribose nucleic acid. G.D. Watson and F.H.C. Crick. Published in Nature, number 4356 April 25, 1953. Nature 248: 765.

Wei, H., M. Scherer, A. Singh, R. Liese, and R. Fischer. 2001. Aspergillus nidulans $\alpha-1,3$ glucanase (mutanase), mutA, is expressed during sexual development and mobilizes mutan. Fungal Genet Biol 34: 217-227.

Wei, N., D.A. Chamovitz, and X.W. Deng. 1994. Arabidopsis COP9 is a component of a novel signaling complex mediating light control of development. Cell 78: 117-124.

Wei, N. and X.W. Deng. 1998. Characterization and purification of the mammalian COP9 complex, a conserved nuclear regulator initially identified as a repressor of photomorphogenesis in higher plants. Photochem Photobiol 68: 237-241.

Wei, N. and X.W. Deng. 1999. Making sense of the COP9 signalosome. A regulatory protein complex conserved from Arabidopsis to human. Trends Genet 15: 98-103.

Weidner, G., B. Steffan, and A.A. Brakhage. 1997. The Aspergillus nidulans lysF gene encodes homoaconitase, an enzyme involved in the fungus-specific lysine biosynthesis pathway. Mol Gen Genet 255: 237-247.

Weidner, G., S. Steidl, and A.A. Brakhage. 2001. The Aspergillus nidulans homoaconitase gene lys $F$ is negatively regulated by the multimeric CCAAT-binding complex AnCF and positively regulated by GATA sites. Arch Microbiol 175: 122-132.

Wek, S.A., S. Zhu, and R.C. Wek. 1995. The histidyl-tRNA synthetase-related sequence in the elF-2 $\alpha$ protein kinase GCN2 interacts with tRNA and is required for activation in response to starvation for different amino acids. Mol Cell Biol 15: 4497-4506.

Wieser, J. and T.H. Adams. 1995. flbD encodes a Myb-like DNA-binding protein that coordinates initiation of Aspergillus nidulans conidiophore development. Genes Dev 9: 491-502.

Winston, F. and P. Sudarsanam. 1998. The SAGA of Spt proteins and transcriptional analysis in yeast: past, present, and future. Cold Spring Harb Symp Quant Biol 63: 553-561.

$\mathrm{Wu}$, J. and B.L. Miller. 1997. Aspergillus asexual reproduction and sexual reproduction are differentially affected by transcriptional and translational mechanisms regulating stunted gene expression. Mol Cell Biol 17: 6191-6201. 
Wu, K., A. Chen, and Z.Q. Pan. 2000. Conjugation of Nedd8 to CUL1 enhances the ability of the ROC1-CUL1 complex to promote ubiquitin polymerization. J Biol Chem 275: 32317-32324.

Xie, Y. and A. Varshavsky. 2000. Physical association of ubiquitin ligases and the 26S proteasome. Proc Natl Acad Sci U S A 97: 2497-2502.

Yager, L.N., H.O. Lee, D.L. Nagle, and J.E. Zimmerman. 1998. Analysis of fluG mutations that affect lightdependent conidiation in Aspergillus nidulans. Genetics 149: 1777-1786.

Yang, R., S.A. Wek, and R.C. Wek. 2000. Glucose limitation induces GCN4 translation by activation of Gen2 protein kinase. Mol Cell Biol 20: 2706-2717.

Yang, X., S. Menon, K. Lykke-Andersen, T. Tsuge, X. Di, X. Wang, R.J. Rodriguez-Suarez, H. Zhang, and N. Wei. 2002. The COP9 signalosome inhibits p27(kip1) degradation and impedes G1-S phase progression via deneddylation of SCF Cul1. Curr Biol 12: 667-672.

Yu, J.H., R.A. Butchko, M. Fernandes, N.P. Keller, T.J. Leonard, and T.H. Adams. 1996a. Conservation of structure and function of the aflatoxin regulatory gene afIR from Aspergillus nidulans and $A$. flavus. Curr Genet 29: 549-555.

Yu, J.H., J. Wieser, and T.H. Adams. 1996b. The Aspergillus FlbA RGS domain protein antagonizes G protein signaling to block proliferation and allow development. EMBO J 15: 5184-5190.

Zhou, C., V. Seibert, R. Geyer, E. Rhee, S. Lyapina, G. Cope, R.J. Deshaies, and D.A. Wolf. 2001. The fission yeast COP9/signalosome is involved in cullin modification by ubiquitin-related Ned8p. BMC Biochem 2: 7.

Zonneveld, B.J. 1975. Sexual differentiation in Aspergillus nidulans: the requirement for manganese and its effect on alpha-1,3 glucan synthesis and degradation. Arch Microbiol 105: 101-104.

Zonneveld, B.J. 1977. Biochemistry and ultrastructure of sexual development of Aspergillus. In Genetics and physiology of Aspergillus. (ed. J.E. Smith and J.A. Pateman), pp. 59-80. Academic Press, London. 


\section{Regulation of the Aspergillus nidulans hisB gene by histidine starvation}

\subsection{Abstract}

The his $B$ gene of the filamentous fungus $A$. nidulans encodes the imidazole glycerol-phosphate dehydratase (IGPD; E.C. 4.2.1.19) catalysing the seventh enzymatic step of histidine biosynthesis. The gene was isolated and it's deduced peptide sequence of 247 amino acids showed up to 54\% identities to IGPD enzymes of organisms comprising all three kingdoms. Expression of hisB cDNA in a Saccharomyces cerevisiae his $3 \Delta$ mutant strain functionally complemented the growth phenotype under histidine limitation. Addition of histidine did not affect his $B$ mRNA levels in $A$. nidulans wild type cells. Histidine starvation conditions increased the his $B$ transcript level fourfold, suggesting regulation by the crosspathway regulatory network. Deletion of the complete hisB open reading frame in the A. nidulans strain A234 resulted in histidine auxotrophy. Additionally, hisB deletion strains were blocked in sexual fruit body formation on medium containing low histidine concentrations. This developmental phenotype of the hisB deletion mutant strain correlated with the induction of the cross-pathway control system.

\subsection{Introduction}

The filamentous fungus Aspergillus nidulans is used as a simple model organism for molecular analysis of interrelated processes in eukaryotes. The ascomycete is particularly advantageous for studies on metabolic multiplicity, genetic networks and multicellular differentiation. Proceeding from multinucleate hyphal growth, complex differentiation processes of asexual and sexual development are induced by environmental signals (Adams et al. 1998). To date several lines of evidence indicate a connection between development and metabolism in A. nidulans (Käfer 1977a; Serlupi-Crescenzi et al. 1983; Eckert et al. 1999).

We aimed to completely delete a histidine biosynthetic gene to investigate whether there is a connection between this amino acid biosynthesis and development in $A$. nidulans. We chose the histidine biosynthetic pathway to discriminate an amino acid specific effect from a broad amino acid effect as supposed by the studies on tryptophan auxotrophs (Eckert et al. 1999). The latter effect is mediated by the cross-pathway control which constitutes a global regulatory network that ensures an increased transcription of a variety of amino acid biosynthetic genes when supply of a single amino acid is limited. This system is common to several filamentous fungi like Neurospora crassa, A. nidulans and 
Aspergillus niger (Carsiotis et al. 1974; Piotrowska 1980) but studied best in Saccharomyces cerevisiae where it is called general control of amino acid biosynthesis. The central transcriptional activator (Gcn4p) of the general control is activated under amino acid starvation conditions via a complex regulatory cascade. Gcn $4 p$ binds to the cis-acting palindromic GCRE (general control response element: 5'-ATGA (C/G) TCAT-3') in the promoters of the amino acid biosynthetic target genes and thus enhances their transcription (Hinnebusch 1997). For the filamentous fungi $N$. crassa and $A$. niger, homologues of the yeast Gcn4p have already been described to act on similar motifs, now designated CPREs for crosspathway response elements (Paluh et al. 1988; Wanke et al. 1997).

None of the genes belonging to the histidine biosynthetic pathway in A. nidulans have been isolated so far. Histidine is synthesised in 10 enzymatic steps from PRPP (phosphoribosyl pyrophosphate), ATP and glutamine in a complex biosynthetic pathway which is biochemically the same in all microorganisms investigated (Alifano et al. 1996). Enforced histidine starvation is commonly used for induction of the cross-pathway control: The false histidine feedback inhibitor 3-amino-1,2,4-triazole (3AT) competitively inhibits the enzyme imidazole glycerol-phosphate dehydratase (IGPD) which results in histidine starvation and subsequent induction of the cross-pathway control (Kanazawa et al. 1988). In the seventh step of histidine biosynthesis, IGPD catalyses the dehydration of imidazole glycerol-phosphate (IGP) to imidazole acetol-phosphate (IAP) (Alifano et al. 1996). Corresponding genes have been isolated from several other organisms. The yeast IGPD encoding gene (HIS3) constitutes a marker commonly used for molecular techniques. In some $\gamma$-proteobacteria the IGPD is a bifunctional enzyme with additional histidinol phosphatase (HPase) activity (Carlomagno et al. 1988; Clark et al. 1998). In none of these organisms deletion of the IGPD encoding genes lead to additional phenotypes except histidine auxotrophy.

In this work we present the isolation of the $A$. nidulans his $B$ gene which encodes the imidazole glycerol-phosphate dehydratase of the histidine biosynthetic pathway. The transcriptional regulation was characterised under various conditions. By deletion of the his $B$ gene a defined loss-of-function mutation of an amino acid biosynthetic gene in $A$. nidulans was constructed. Investigations of the mutant's sexual reproductive cycle identified a strong correlation between an induced cross-pathway control and a block in sexual development at the level of micro-cleistothecia. In contrast, overproduction of HISB did not show any effect on the development of the fungus. 


\subsection{Experimental procedures}

\subsubsection{Strains and media}

Yeast strains H1515 (leu2, -112 ura3-52 trp1 GAL2; obtained from A. Hinnebusch; Bethesda, USA) and RH2037 (ura3-1, trp1-1, ade2-1, leu2-3,112, his3-11,15, rna15-2, GAL2) (Minvielle-Sebastia et al. 1991) were cultivated on minimal medium with $2 \%$ glucose or galactose as sole carbon source (Miozzari et al. 1978).

The $A$. nidulans strain FGSC A234 ( $y A 2$, pabaA1; veA1) was provided by the Fungal Genetics Stock Center (University of Kansas Medical Center, Kansas City, KS, USA). Strain GR5 (wA3; pyrG89; pyroA4; veA1) was obtained from G. May (Houston, USA). A. nidulans strains were cultivated at $30^{\circ} \mathrm{C}$ on minimal medium supplemented as described (Käfer 1977b; Bennett and Lasure 1991). The standard concentration of histidine in the medium was $0.3 \mathrm{mM}$ except where noted. For amino acid starvation, mycelia of liquid overnight precultures were adapted for $1 \mathrm{~h}$ in fresh medium before $20 \mathrm{mM}$ 3-amino-1,2,4-triazole (3AT) was added. On solid medium supplemented with $2 \mathrm{mM} 3 \mathrm{AT}$, conidia were inoculated on sterile miracloth filters to enable the transfer to fresh 3AT plates in two-day intervals. For enhanced cleistothecia development, plates were incubated under oxygen-limiting conditions by wrapping petri dishes with tape (Clutterbuck 1974). For construction of the his $B$ deletion mutant, the $B a m H \mathrm{H} / K p n l$-fragment of plasmid pME1657 was transformed into A. nidulans strain A234 as described earlier (Eckert et al. 2000). 14 transformants were obtained upon selection for the presence of the ble gene of Streptoalloteichus hindustanus on minimal medium containing $10 \mu \mathrm{g} / \mathrm{ml}$ phleomycin (Cayla, France). Single homologous recombination events were proven by PCR of the transformant's genomic DNA and by Southern hybridisation experiments (Fig. 2.2). Two transformants contained exclusively the hisB deletion and both strains showed exactly the same phenotypic effects in all respects. One of them was strain AGB40. For construction of the hisB overexpression strain, plasmid pME1764 was transformed into A. nidulans GR5 and selected for the plasmid's pyrG marker on minimal medium lacking uridine. Eight of the transformants contained additional his $B$ copies in the genome as detected by Southern hybridisation experiments. A. nidulans strain AGB75 contained two additional copies of the his $B$ gene. Increased his $B$ transcript levels were verified by Northern experiments under ethanol induced conditions.

\subsubsection{Isolation of hisB and plasmid construction}

Full length cDNA clones of hisB were isolated from an $A$. nidulans inducible GAL1driven cDNA expression library as described (Krappmann et al. 1999; Hoffmann et al. 2000a) via functional complementation of the yeast his3s mutant strain RH2037 on medium with galactose as sole carbon source but lacking histidine. Yeast cells were transformed as described earlier (Ito et al. 1983). In six out of 10,000 yeast transformants histidine prototrophy was restored and the isolated plasmids revealed an identical cDNA sequence resulting in plasmid pME1511. For isolation 
of the genomic hisB clone, a Xhol-digested genomic DNA sublibrary of $A$. nidulans was subcloned into pBluescriptSK ${ }^{+}$and transformed into E. coli for colony hybridisation using the CDNA fragment as probe which revealed plasmid pME1512. For construction of the deletion cassette pME1512 was amplified via PCR with primers excluding the hisB coding region which was replaced by the Sspl-cut phleomycin resistance cassette of plasmid pAN8-1 (Punt and van den Hondel 1992) via blunt end ligation resulting in plasmid pME1657 (Fig. 2.2). The overexpression cassette in plasmid pME1764 was constructed by blunt end ligation of the hisB cDNA (Sall/Notl fragment of pME1511) to the alcA-promoter (Smal) of pME1565 (Hoffmann et al. 2000a).

\subsubsection{Recombinant DNA techniques and computational analyses}

Standard molecular methods for DNA isolation and manipulation were applied according to Sambrook (Sambrook et al. 1989). For Northern hybridisation analysis, total RNAs were isolated with TRIZOL Reagent (Gibco BRL). Total RNA (20 $\mu$ g per lane) were separated on a formaldehyde agarose gel, electroblotted on a nylon membrane (Biodyne, PALL) and hybridised with ${ }^{32}$ P-labeled DNA probes (Feinberg and Vogelstein 1984). The RNA-ladder of Gibco BRL was used as RNA size standard. Signal intensities were quantified with a bioimager using the program MacBAS v2.5(E) from Fuji.

For DNA sequence analysis with custom oligonucleotides (Gibco BRL) plasmid DNA was labelled with the Big Dye ${ }^{\mathrm{TM}}$ Terminator kit of the Cycle Sequencing Reaction Mix from Perkin Elmer. Sequencing was performed by a Perkin Elmer Sequenator 310 (Norwalk, CT, USA) with sequence analysis program $A B I$ EditView 1.0.1. Further DNA analysis was performed with the Lasergene Navigator software from DNASTAR (Madison, WC, USA). IGPD sequences were identified using BLAST search of a non-redundant protein database (Altschul et al. 1990). Pairwise alignments and the multiple sequence alignment with hierarchial clustering was performed using the software "CLUSTALW" of F. Corpet (Corpet 1988). Putative transcription factor binding sites were identified with TFSEARCH vs 1.3 (Heinemeyer et al. 1998) at the Real World Computing Partnership/Parallel Application TRC Lab. (http://pdap1.trc.rwcp.or.jp/research/db/TFSEARCH.html). The sequence data of the genomic fragment (4.347 kb of plasmid pME1512) containing the complete hisB gene have been submitted to the GenBank database under accession number AF246264.

\subsubsection{Microscopic analysis}

Growth, conidiation and cleistothecia development of $A$. nidulans were monitored under a Zeiss Stemi 2000-C binocular (Jena, Germany). For detailed microscopical analysis a Zeiss Axiovert S100 microscope (Jena, Germany) with Nomarski-DIC optics was used. For fluorescence microscopy, cleistothecia and microcleistothecia were carefully pressed under a cover slide and stained with 4,6diamidino-2-phenylindole (DAPI, Sigma) according to Pringle (Pringle et al. 1991). 


\section{$2.4 \quad$ Results}

\subsubsection{The hisB gene of Aspergillus nidulans encodes the imidazole glycerol- phosphate dehydratase of the histidine biosynthetic pathway.}

The yeast HIS3 gene encodes the imidazole glycerol-phosphate dehydratase [IGPD, E.C. 4.2.1.19] essential for histidine biosynthesis. The cDNA of the corresponding $A$. nidulans his $B$ gene was isolated by functional complementation of the histidine auxotrophy of the Saccharomyces cerevisiae his3s strain RH2037. Sequence analysis of the $980 \mathrm{bp}$ cDNA revealed an open reading frame of $741 \mathrm{bp}$. In Southern hybridisation experiments the isolated cDNA used as probe hybridised to a single $6.2 \mathrm{~kb}$ Xhol genomic fragment. Thus, only a single copy of the cDNA encoding gene is present in the genome of $A$. nidulans (data not shown). The corresponding $6.2 \mathrm{~kb}$ genomic fragment was isolated and contained an open reading frame identical to the one of the cDNA clone, flanked by a $2.4 \mathrm{~kb} 5$ '- and a $3.0 \mathrm{~kb} 3$ '-region. The open reading frame was interrupted $319 \mathrm{bp}$ downstream of the translational start point by one intron of $59 \mathrm{bp}$ showing the conserved 5'splicing, internal- and 3'-splicing sequences described for introns of $A$. nidulans (May et al. 1987). The cDNA clone marked the poly $(A)$ site $150 \mathrm{bp}$ downstream of the UAA stop codon.

From the deduced peptide sequence of 247 amino acids a molecular weight of $26.2 \mathrm{kD}$ was calculated. The polypeptide sequence shows high identities to IGPDs of organisms comprising representatives of all three kingdoms. The multialignment in Fig. 2.1 presents a selection of IGPD amino acid sequences from other fungi, plants, gram positive and negative bacteria as well as archea. All of them, including the deduced protein sequence of $A$. nidulans, contain the well conserved ' $\mathrm{dHHxxE}$ ' domains characteristic for imidazole glycerol-phosphate dehydratases (Parker et al. 1994). The identities of up to $54 \%$ to genes of other IGPDs and the complementation of the yeast his $3 \Delta$ mutant strain characterises the isolated cDNA as imidazole glycerol-phosphate dehydratase encoding gene of $A$. nidulans. Therefore the corresponding gene was named his $B$.

\subsubsection{Construction of an $A$. nidulans his $B$ deletion strain auxotroph for histidine.}

We constructed a his $B$ deletion strain to analyse the function of his $B$ in the filamentous fungus. The complete his $B$ open reading frame of the haploid A. nidulans wild-type strain $\mathrm{A} 234$ was replaced by a phleomycin-resistance cassette. Our deletion strain AGB40 contained the resistance cassette as single integration at the original genomic his $B$ locus due to homologous integration of the hisB flanking DNA regions (Fig. 2.2). In Northern experiments using the hisB cDNA as a probe, no hisB transcript was detected for strain AGB40 (data not shown). On minimal medium without histidine, germination and growth were totally blocked in the hisB deletion strain AGB40. Supplementation of at least $0.3 \mathrm{mM}$ histidine to the growth medium facilitated germination, vegetative growth and conidiation of the 
hisB deletion strain alike the wild-type. Transformation of the $6.2 \mathrm{~kb}$ genomic fragment containing the his $B$ gene into the his $B$ deletion strain completely restored histidine prototrophy. Even a Bgll / Kpnl fragment of the genomic locus, a truncated 5 '-version containing only $730 \mathrm{bp}$ of the upstream region, functionally complemented the histidine auxotrophy of AGB40.

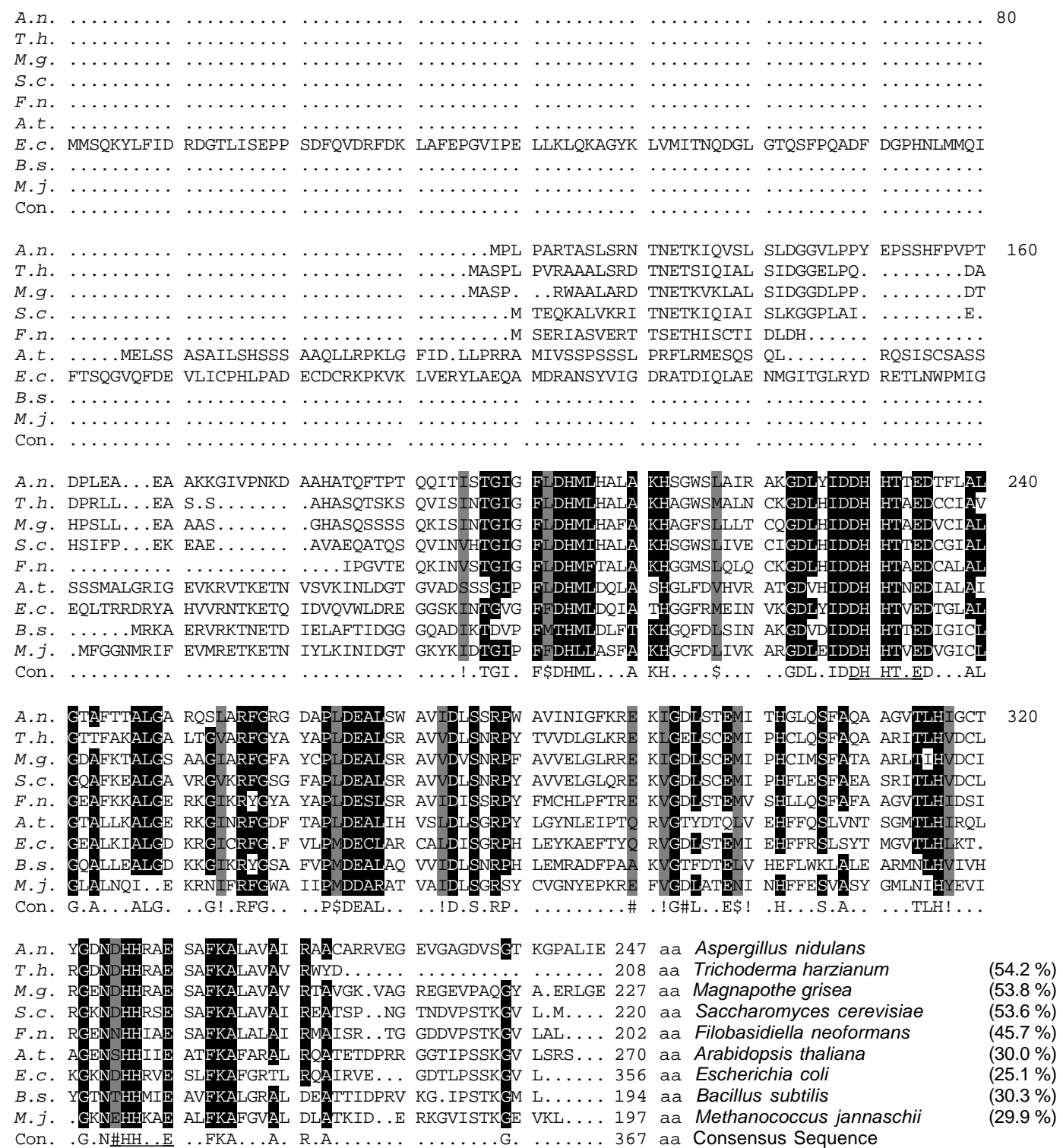

Fig. 2.1: Amino acid comparison of IGPDs. Imidazole glycerol-phosphate dehydratase protein sequences of organisms comprising all three kingdoms were aligned. Accession numbers of the swissprot database are: p34041 (T. harzianum); 042621 (M. grisea); p06633 (S. cerevisiae); p40919 ( $F$ neoformans); p34047 (A. thaliana); p10368 (E. coll); 034683 (B. subtilis) and q58109 (M. janaschii). The percentage of identity of each IGPD sequence to the $A$. nidulans HISB is given in parentheses. Identical amino acids in minimal seven of the sequences are highlighted in black boxes. Grey shading indicates amino acids of high similarity $(!=I, V ; \$=L, M ; \%=F Y ; \#=N, D, Q, E, B)$. The highly conserved "HHxxE" domains are shown underlined in the consensus sequence. 


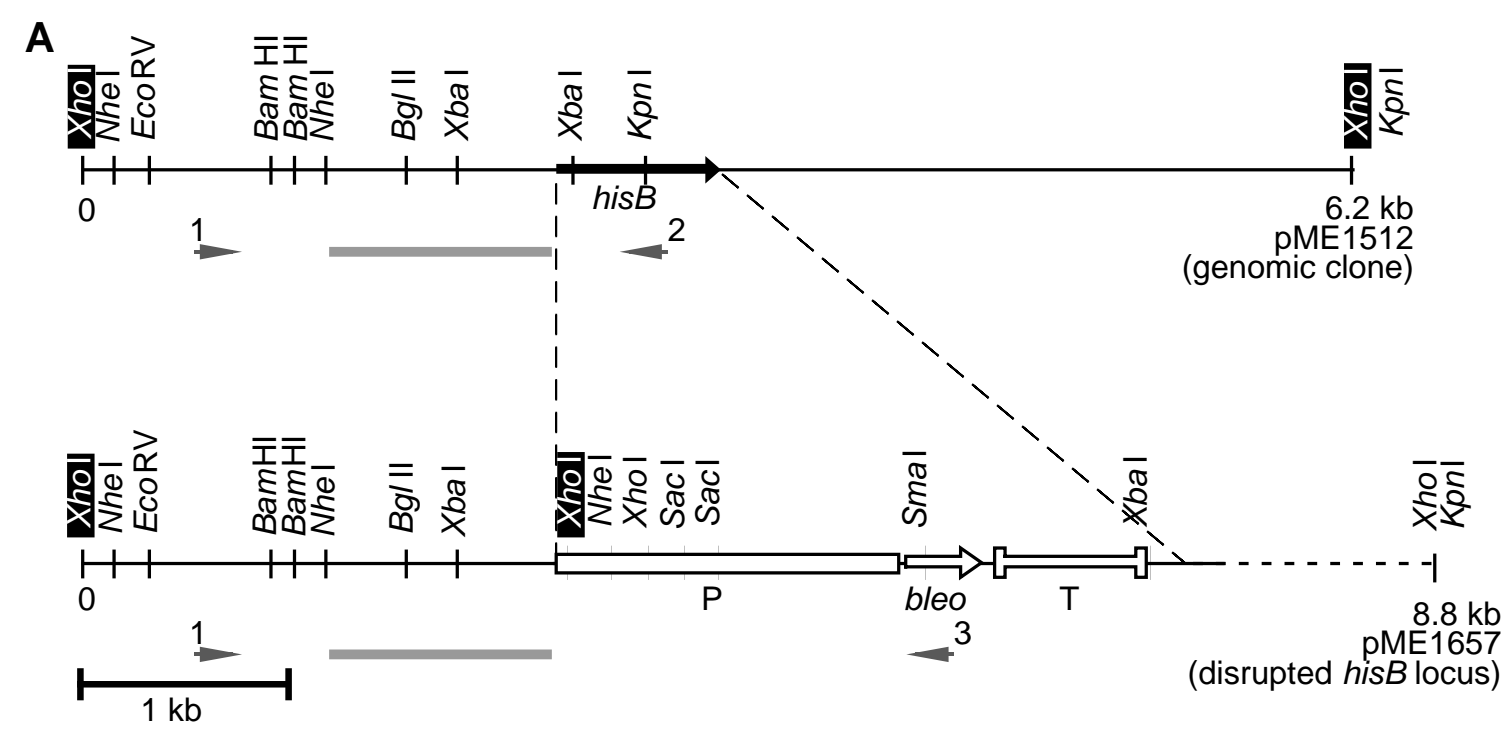

B

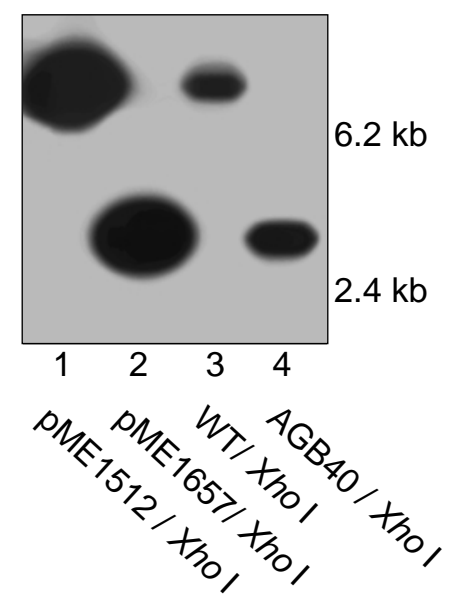

Fig. 2.2: Construction of the Aspergillus nidulans his $B$ deletion strain AGB40. (A) The complete open reading frame of the $A$. nidulans his $B$ gene on plasmid pME1512 was replaced by a phleomycin resistance cassette (PgpdA-promoter, bleo phleomycin marker, TtrpC-terminator). For transformation of $A$. nidulans strain A234, the BamHI/Kpnl fragment of the hisB deletion cassette was used. Transformants were screened for homologous integration by PCR with three different primers in parallel (grey arrows). (B) Integration of the phleomycin resistance cassette was verified by Southern analysis with the hisB upstream region as a probe (grey bar). The autoradiography shows Xhol-digestion of genomic wild-type and AGB40 DNA (lines 3,4) and of plasmids pME1512 and pME1657 (lines 1,2); and the relevant Xhol sites are indicated by black boxes.

\subsubsection{The his $B$ gene of $A$. nidulans is regulated on the transcriptional level under amino acid starvation conditions}

The promoter sequence of his $B$ of $A$. nidulans contains several putative regulatory elements. Two poly-d(AT) rich regions (5'-TTTTGAAAAAT-3' and 5'-TTTTATTATT-3') at positions -175 and -135 might function as TATA-elements for general transcription (Winter and Varshavsky 1989). Another two represent possible binding sites for developmental and amino acid specific transcription factors: A putative STRE ( StuA response element) is located at position -182: 5'-ACGCGGGA3 ' and a site similar to yeast's GCRE (general control response element) at position -229: 5'-ATGACGCA-3'.

STREs constitute specific binding sites for the transcription factor STUA and are generally found upstream of genes important for development or cell cycle in A. nidulans. Genes under control of this transcription factor have been shown to be regulated during development (Miller et al. 1991; Dutton et al. 1997). Northern experiments showed equal levels of approximately $1 \mathrm{~kb}$ hisB mRNAs during vegetative growth, asexual and sexual spore formation (data not shown). This 
A

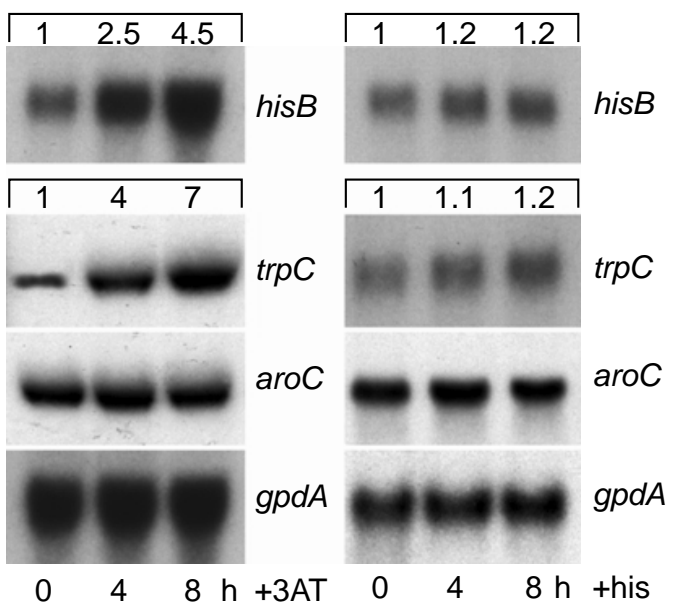

Fig. 2.3: Transcriptional regulation of the A. nidulans hisB gene. Mycelia were grown in liquid culture, either (A) under induction of the cross-pathway control by $20 \mathrm{mM} 3$-amino1,2,4-triazole (3AT) or (B) at an excessive histidine (his) concentration of $30 \mathrm{mM}$. RNA was isolated at the time points indicated and probed with the hisB gene in Northern hybridisation experiments. Controls: trpC (regulated by the cross-pathway control), aroC and $g p d A$ (both transcribed constitutively under the conditions tested). For quantification, mRNA amounts were equalised according to the level of $g p d A$ transcription and mRNA amounts of hisB at the time point 0 were set as 1 . Relative his $B$ and trpC mRNA levels are given.

suggested that hisB transcription is normally not affected during development. Thus, the putative binding site for the STUA protein in the promoter region of his $B$ does not seem to play an important role during development.

The GCRE-like site exhibits high similarity to the binding site of the general control transcriptional activator Gcn4p of yeast (Hinnebusch 1988). The consensus sequences between the Gcn4p protein and its homologue of $A$. nidulans might be similar because the genes $\arg B, \operatorname{trp} B$ and the $\operatorname{trp} C$ of $A$. nidulans subjected to this regulatory mechanism show identical sequence motifs in their promoter regions (Mullaney et al. 1985; Goc and Weglenski 1988; Eckert et al. 2000) which are called CPRE (cross-pathway response element) in filamentous fungi. The CPRE site in the promoter region of his $B$ seems to be important under amino acid starvation conditions. We determined the level of his $B$ transcription upon addition of the histidine analogue 3-amino-1,2,4-triazole (3AT) in Northern hybridisation experiments. 3AT acts as false feedback-inhibitor of the HISB protein and thus causes histidine starvation with subsequent induction of the cross-pathway control. Eight hours after $3 A T$ addition, the his $B$ transcript level increased about fourfold compared to cultures not incubated with 3AT (Fig. 2.3A). Similar results were found for the trpC gene [E.C. 2.1.3.3] encoding a trifunctional tryptophan biosynthetic enzyme known to be regulated by the cross-pathway control (Eckert et al. 1999). An amino acid biosynthetic gene unaffected by the regulatory network, aroC, showed constitutive expression (Krappmann et al. 1999). We examined whether the effect of 3AT on hisB transcription is reversed by the simultaneous addition of histidine to exclude that the analogue or histidine have a specific effect on his $B$ transcription. A. nidulans cells were simultaneously supplemented with $20 \mathrm{mM}$ histidine and $10 \mathrm{mM} 3 \mathrm{AT}$, resulting in stable basal his $B$ mRNA levels during cultivation (data not shown). We also tested whether the transcription of his $B$ is affected at excessive histidine supplementation $(30 \mathrm{mM})$ without the analogue. his $B$ mRNA levels of an $A$. nidulans wild-type strain grown on high histidine concentrations after shift from minimal medium without histidine remained on a low level (Fig. 2.3B). These data suggest that on transcriptional level the his $B$ gene of $A$. nidulans is regulated by the cross-pathway control but not by an additional histidine-specific regulatory mechanism. 


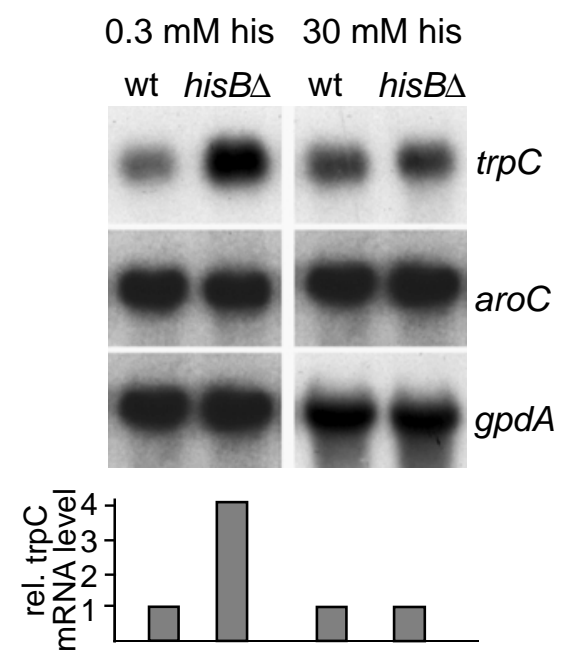

Fig. 2.4: Induction of the crosspathway control in a his $B \Delta$ mutant strain. A. nidulans wild-type strain A234 and the hisB deletion mutant AGB40 were grown overnight in liquid minimal medium containing $0.3 \mathrm{mM}$ or $30 \mathrm{mM}$ histidine (his). RNA was isolated and the status of the cross-pathway control was monitored using the trpC gene as a probe in Northern hybridisation experiments (controls as in Fig. 2.3). mRNA levels were equalised according to the gpdA transcriptional level and mRNA amounts of wild-type were set as 1. Relative trpC mRNA levels are given.

\subsubsection{Growth on low histidine concentration causes induction of the cross-pathway control in the hisB deletion mutant.}

The histidine analogue 3AT reduced the activity of the HISB protein by false feedback inhibition and therefore induced a shortage of histidine. This starvation for histidine induced the cross-pathway control which subsequently increased his $B$ transcription. Low concentrations of histidine should result in a similar phenotype in an $A$. nidulans hisB mutant strain in the absence of 3AT. Therefore we tested transcription of the cross-pathway control-regulated trpC gene (Eckert et al. 1999) in the hisB mutant AGB40 after incubation in liquid minimal medium which allows exclusively growth of vegetative mycelium. Under low histidine concentrations (0.3 and $3 \mathrm{mM}$ ), the mRNA level of trpC was increased about fourfold in the mutant compared to the wild-type strain. Under high concentrations of histidine (30 and $60 \mathrm{mM}$ ) no difference in mRNA levels was observed between the his $B$ mutant and the wild-type strain (Fig. 2.4). The same results were found for $\operatorname{argB}$, a second cross-pathway regulated gene (data not shown). The aroC gene which is unaffected by the cross-pathway control showed constant mRNA levels under any condition tested. The same results were obtained in Northern experiments with RNA from cultures grown on solid medium at different developmental time points (data not shown). These data indicate that supplementation of the hisB deletion mutant with low concentrations of histidine activates the cross-pathway regulatory network.

\subsubsection{Deletion of $\boldsymbol{h i s} B$ affects sexual development of $\boldsymbol{A}$. nidulans.}

A. nidulans strains with point mutations in tryptophan amino acid biosynthetic genes showed an aberrant sexual development dependent on the tryptophan concentration supplied to the medium (Eckert et al. 1999). We used our A. nidulans his $B$ deletion strain to analyse whether the sexual development is impaired in this strain with a defined loss-of-function mutation of a different amino acid biosynthetic 
gene. Sexual development of the wild-type (strain A234) was independent of the histidine concentration supplied $(0.3 \mathrm{mM}, 3 \mathrm{mM}, 30 \mathrm{mM}$ and $60 \mathrm{mM})$. After induction of at least $60 \mathrm{~h}$ of incubation nests were the first visible sexual structures. Small, soft and pale coloured micro-cleistothecia were formed within the nests after $90 \mathrm{~h}$. The maturation of the sexual fruit bodies was completed another $110 \mathrm{~h}$ later resulting in hard, darkly red coloured cleistothecia filled with fertile ascospores. In the hisB deletion strain AGB40 sexual development was unaffected for the first $90 \mathrm{~h}$ and thus micro-cleistothecia were formed at numbers similar to wild-type at any histidine concentration tested. On medium containing high concentrations of histidine (30 or $60 \mathrm{mM}$ ) the hisB mutant strain developed wild-type-like mature cleistothecia after $200 \mathrm{~h}$. In contrast, the sexual cycle was blocked under conditions of low histidine concentrations $(0.3$ and $3 \mathrm{mM})$ at the reproducible time point of micro-cleistothecia formation without any further maturation even after $200 \mathrm{~h}$ (Fig. 2.5A, B). Microscopic analysis showed that the micro-cleistothecia of the hisB deletion mutant grown at $0.3 \mathrm{mM}$ histidine formed a small inner cavity, entirely filled with hyphae instead of ascospores after 90 and 200 hours of incubation. In the wild-type, this developmental state of micro-cleistothecia was an intermediate state after 90 hours which then continued to grow to well-rounded mature cleistothecia containing ascospores after an incubation of $200 \mathrm{~h}$ (Fig. 2.5C). These observations were confirmed by DAPI staining of hyphal nuclei. After $90 \mathrm{~h}$, the nuclei in the hyphae of wild-type and hisB mutant micro-cleistothecia are stained bright blue. Completion of the sexual cycle after $200 \mathrm{~h}$ prevents staining by formation of ascospores in the wild-type, but not in the hisB mutant strain on low histidine concentrations (data not shown). When the deletion strain AGB40 is complemented with the hisB wild type genomic fragment (plasmid pME1512 integrated ectopically in the genome) normal cleistothecia develop at any histidine concentration tested. These data indicate a connection between histidine biosynthesis and sexual development in $A$. nidulans.

Micro-cleistothecia of the hisB mutant strain which were blocked in their development by low histidine supplementation were further analysed to distinguish whether the arrest is irreversible or whether development can be completed in a changed environment. Therefore micro-cleistothecia, which were grown for 200 hours on medium containing $0.3 \mathrm{mM}$ histidine, were shifted to media containing 30 and $60 \mathrm{mM}$ histidine and cultivated for additional 120 hours. During this time, micro-cleistothecia released the block and completed development resulting in normal cleistothecia with fertile ascospores (data not shown). Thus, $90 \mathrm{~h}$ after induction of the sexual developmental process, a control point exists to decide whether the sexual cycle should be completed.

The effect of blocked sexual reproduction in the $A$. nidulans his $B$ deletion strain prompted us to check the developmental capacity of a HISB overproducing $A$. nidulans strain. Therefore, his $B$ was fused to the inducible alcA-promoter and ectopically integrated into the genome of $A$. nidulans GR5. Strain AGB75 showed up to 30 fold increased transcript levels as proven by Northern experiments (data not shown). The functionality of the hisB overproduction was checked on solid minimal medium containing $10 \mathrm{mM}$ 3AT. These high 3AT concentration prevents 
$0.3 \mathrm{mM}$ histidine

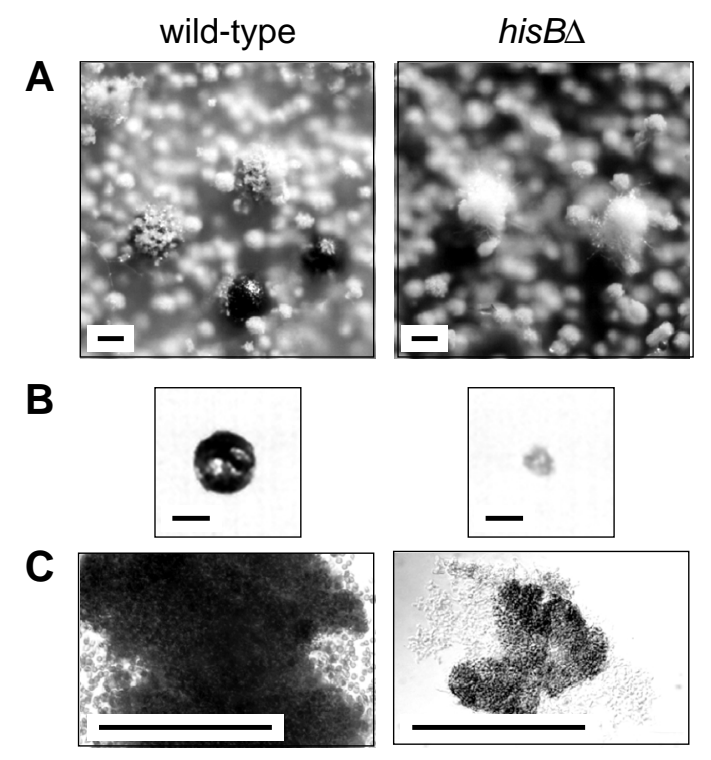

$30 \mathrm{mM}$ histidine

wild-type
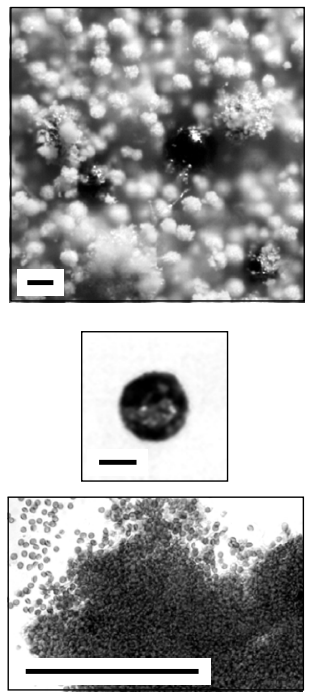

hisB $\Delta$
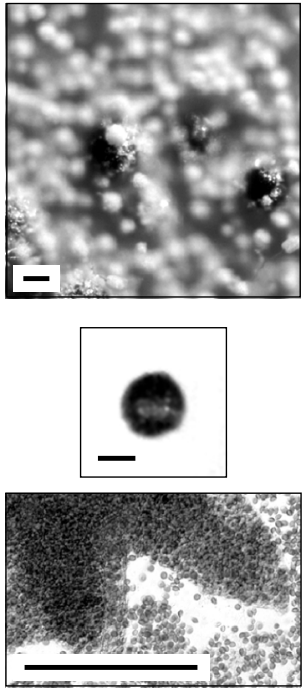

Fig. 2.5: Deletion of the $A$. nidulans his $B$ gene blocks sexual development under low histidine concentrations. Sexual development of the wild-type strain AGB234 and the his $B$ deletion strain AGB40 were investigated on agar plates containing low $(0.3 \mathrm{mM})$ or high $(30 \mathrm{mM})$ histidine concentrations. The wild-type develops mature cleistothecia under all conditions tested. So does the mutant under high histidine concentrations. At low histidine concentrations, the mutant AGB40 stops the differentiation process after about $90 \mathrm{~h}$ development, leading to palecoloured, soft and small micro-cleistothecia. The morphology of the sexual reproductive structures is shown under a binocular in colony context $(\mathbf{A})$ and as isolated structures $(B)$. The structures were slightly pressed under a cover slide and studied under the light microscope: the mature cleistothecia are filled with ascospores whereas the micro-cleistothecium is filled with hyphae (C). Pictures were taken after an incubation time of $200 \mathrm{~h}$. Scale bars approx. $100 \mu \mathrm{m}$.

growth of the wild-type but allows growth of strains with a surplus of the analogs' target enzyme HISB. All transformants grew under conditions with $10 \mathrm{mM}$ 3AT. But none of the his $B$ overproducing $A$. nidulans strains did show any significant morphological or developmental aberration during asexual or sexual life cycle when grown under inductive conditions with ethanol as the sole carbon source.

\subsection{Discussion}

In this work we present the first isolation of a histidine biosynthetic gene of the filamentous fungus $A$. nidulans. Complete deletion of the his $B$ coding region uncovered a block in the sexual development of the mutant strain at low histidine supplementation. Detailed analysis of hisB transcript levels showed a correlation of this developmental phenotype with activation of the cross-pathway regulatory system. Overproduction of the HISB protein did not show any effect on the development of the fungus.

We demonstrated that deletion of the complete his $B$ open reading frame results in histidine auxotrophy. Retransformation of the his $B$ gene into the deletion mutant AGB40 completely restored histidine prototrophy. Thus, the his $B$ gene can be easily used as selectable marker in $A$. nidulans. Since $A$. nidulans is highly sensitive for $3 A T$, the HISB protein as target of this inhibitor might be specifically used to reveal the binding site of 3AT (Kanazawa et al. 1988) within the enzyme. 
The HISB protein sequence showed up to $54 \%$ identity to imidazole glycerolphosphate dehydratases of other organisms which possibly indicates homology of these proteins. IGPD-encoding genes of archea, bacteria and eukarya have been cloned by complementation of the corresponding E. coli, B. subtilis or S. cerevisiae mutants indicating functional conservation of the IGPD enzymes, even though the IGPD enzymes of $\gamma$-proteobacteria are combined with an additional histidinol phosphatase-activity (Alifano et al. 1996). This further counts for a common origin of all IGPD encoding genes of histidine biosynthesis.

Computational analysis of the A. nidulans hisB promoter region revealed several putative regulatory elements including possible targets for a general transcription factor as well as for developmental and amino acid specific transcription factors. The two poly-d(AT)-stretches are similar to the TATA elements (Tc and Tr) in the S. cerevisiae HIS3 promoter which direct constitutive and activator-dependent transcription initiation to the sites +1 and +13 , respectively (lyer and Struhl 1995). We showed that the putative STRE site in the hisB promoter seems either not functional or has a function not known to date. The transcription factor STUA, site-specific for STREs, is important for regulation of multicellular development in $A$. nidulans. It is involved in asexual development and presumably in the induction of the sexual reproductive cycle in $A$. nidulans (Dutton et al. 1997). If the proposed STUA binding site in the hisB 5'-regulatory region was functional, hisB transcription should be regulated during development. However, under standard growth conditions the his $B$ transcript levels of the A. nidulans wild-type A234 were not significantly changed during hyphal growth, the asexual or the sexual phase of A. nidulans. We demonstrated that hisB is transcriptionally regulated under amino acid starvation conditions which indicates that the CPRE in the promoter region is functional. The corresponding central transcription factor of the cross-pathway control should be homologous to the yeast Gcn4p and the corresponding homologues Cpc-1p and CpcAp of the filamentous fungi $N$. crassa and A. niger (Hinnebusch 1988; Paluh et al. 1988; Wanke et al. 1997). The homologous HIS3 gene of $S$. cerevisiae contains even three GCREs in the regulatory region and shows similar rates of induction by amino acid starvation (Struhl 1982; Hope and Struhl 1985; Hinnebusch 1988).

When the hisB deletion strain AGB40 was grown on low histidine concentrations two phenomena became obvious: the sexual development was blocked on the level of micro-cleistothecia and simultaneously the cross-pathway control was induced. The same strain grown under conditions of high histidine supplementation differentiates mature, fertile cleistothecia and the cross-pathway control is repressed, just like the wild-type on any histidine supplementation tested. These findings indicate a cross-connection between completion of the sexual development and an activated cross-pathway control. This raises the question whether the block of sexual development is a histidine specific effect or a general amino acid deficiency effect mediated by the cross-pathway control. The latter presumption is supported by previous findings: mutations in tryptophan biosynthetic genes of $A$. nidulans resulted in the same block of sexual development and an activated cross-pathway control as shown for our hisB-deletion strain 
(Eckert et al. 1999). Thus, all data confirm that the cross-pathway control is turned on due to amino acid starvation under conditions where no mature cleistothecia are build. Additionally, overexpression of the cross-pathway central transcription factors of $A$. niger and $N$. crassa in an $A$. nidulans wild-type strain resulted in a similar block of sexual development at the level of micro-cleistothecia (Hoffmann et al. 2000b). Apart from that, over-supplementation of $A$. nidulans wild-type strains with tryptophan or arginine impaired sexual development (Serlupi-Crescenzi et al. 1983; Eckert et al. 1999). For histidine, we did not identify any concentration that affects sexual development of the wild-type. Possibly the cross-pathway control is not turned on by over-supplementation with histidine due to a limited uptake of histidine. In contrast to the $A$. nidulans $\operatorname{trp} C$ and $\arg B$ mutants, where insufficient tryptophan and arginine supply, respectively, affected asexual development, no effect on conidiation was observed for the his $B$ deletion strain upon supplementation with low levels of histidine. For our hisB deletion strain AGB40 the block in sexual development seems to be exclusively caused by induction of the cross-pathway control.

\subsection{References}

Adams, T.H., J.K. Wieser, and J.H. Yu. 1998. Asexual sporulation in Aspergillus nidulans. Microbiol Mol Biol Rev 62: 35-54.

Alifano, P., R. Fani, P. Lio, A. Lazcano, M. Bazzicalupo, M.S. Carlomagno, and C.B. Bruni. 1996. Histidine biosynthetic pathway and genes: structure, regulation, and evolution. Microbiol Rev 60: 44-69.

Altschul, S.F., W. Gish, W. Miller, E.W. Myers, and D.J. Lipman. 1990. Basic local alignment search tool. $J$ Mol Biol 215: 403-410.

Bennett, J.W. and L.L. Lasure. 1991. Growth media. In More gene manipulation in fungi (ed. J.W. Bennett and L.L. Lasure), pp. 441-457. Academic Press Inc, San Diego.

Carlomagno, M.S., L. Chiariotti, P. Alifano, A.G. Nappo, and C.B. Bruni. 1988. Structure and function of the Salmonella typhimurium and Escherichia coli K-12 histidine operons. J Mol Biol 203: 585-606.

Carsiotis, M., R.F. Jones, and A.C. Wesseling. 1974. Cross-pathway regulation: histidine-mediated control of histidine, tryptophan, and arginine biosynthetic enzymes in Neurospora crassa. J Bacteriol 119: 893898.

Clark, M.A., L. Baumann, and P. Baumann. 1998. Buchnera aphidicola (Aphid endosymbiont) contains genes encoding enzymes of histidine biosynthesis. Curr Microbiol 37: 356-358.

Clutterbuck, A.J. 1974. Aspergillus nidulans. In Handbook of Genetics. (ed. R.C. King), pp. 447-510. Plenum, New York.

Corpet, F. 1988. Multiple sequence alignment with hierarchical clustering. Nucleic Acids Res 16: 10881-10890.

Dutton, J.R., S. Johns, and B.L. Miller. 1997. StuAp is a sequence-specific transcription factor that regulates developmental complexity in Aspergillus nidulans. EMBO J 16: 5710-5721.

Eckert, S.E., B. Hoffmann, C. Wanke, and G.H. Braus. 1999. Sexual development of Aspergillus nidulans in tryptophan auxotrophic strains. Arch Microbiol 172: 157-166.

Eckert, S.E., E. Kübler, B. Hoffmann, and G.H. Braus. 2000. The tryptophan synthase-encoding trpB gene of Aspergillus nidulans is regulated by the cross-pathway control system. Mol Gen Genet 263: 867-876.

Feinberg, A.P. and B. Vogelstein. 1984. A technique for radiolabeling DNA restriction endonuclease fragments to high specific activity. Anal Biochem 137: 266-267.

Goc, A. and P. Weglenski. 1988. Regulatory region of the Aspergillus nidulans argB gene. Curr Genet 14: 425-429.

Heinemeyer, T., E. Wingender, I. Reuter, H. Hermjakob, A.E. Kel, O.V. Kel, E.V. Ignatieva, E.A. Ananko, O.A. Podkolodnaya, F.A. Kolpakov, N.L. Podkolodny, and N.A. Kolchanov. 1998. Databases on transcriptional regulation: TRANSFAC, TRRD and COMPEL. Nucleic Acids Res 26: 362-367. 
Hinnebusch, A.G. 1988. Mechanisms of gene regulation in the general control of amino acid biosynthesis in Saccharomyces cerevisiae. Microbiol Rev 52: 248-273.

Hinnebusch, A.G. 1997. Translational regulation of yeast GCN4. A window on factors that control initiatortRNA binding to the ribosome. J Biol Chem 272: 21661-2164.

Hoffmann, B., S.K. LaPaglia, E. Kubler, M. Andermann, S.E. Eckert, and G.H. Braus. 2000a. Developmental and metabolic regulation of the phosphoglucomutase- encoding gene, pgmB, of Aspergillus nidulans. Mol Gen Genet 262: 1001-1011.

Hoffmann, B., C. Wanke, S.K. da Paglia, and G.H. Braus. 2000b. c-Jun and RACK1 homologues regulate a control point for sexual development in Aspergillus nidulans. Mol Micobiol 37: 1-15.

Hope, I.A. and K. Struhl. 1985. GCN4 protein, synthesised in vitro, binds HIS3 regulatory sequences: implications for general control of amino acid biosynthetic genes in yeast. Cell 43: 177-188.

Ito, H., Y. Fukuda, K. Murata, and A. Kimura. 1983. Transformation of intact yeast cells treated with alkali cations. J Bacteriol 153: 163-168.

lyer, V. and K. Struhl. 1995. Mechanism of differential utilization of the HIS3 $\mathrm{T}_{\mathrm{R}}$ and $\mathrm{T}_{\mathrm{C}}$ TATA elements. Mol Cell Biol 15: 7059-7066.

Käfer, E. 1977a. The anthranilate synthetase enzyme complex and the trifunctional trpC gene of Aspergillus. Can J Genet Cytol 19: 723-738.

Käfer, E. 1977b. Meiotic and mitotic recombination in Aspergillus and its chromosomal aberrations. Adv Genet 19: 131-133.

Kanazawa, S., M. Driscoll, and K. Struhl. 1988. ATR1, a Saccharomyces cerevisiae gene encoding a transmembrane protein required for aminotriazole resistance. Mol Cell Biol 8: 664-673.

Krappmann, S., K. Helmstaedt, T. Gerstberger, S. Eckert, B. Hoffmann, M. Hoppert, G. Schnappauf, and G.H. Braus. 1999. The aroC gene of Aspergillus nidulans codes for a monofunctional, allosterically regulated chorismate mutase. J Biol Chem 274: 22275-22282.

May, G.S., M.L. Tsang, H. Smith, S. Fidel, and N.R. Morris. 1987. Aspergillus nidulans beta-tubulin genes are unusually divergent. Gene 55: 231-243.

Miller, K.Y., T.M. Toennis, T.H. Adams, and B.L. Miller. 1991. Isolation and transcriptional characterisation of a morphological modifier: the Aspergillus nidulans stunted (stuA) gene. Mol Gen Genet 227: 285-292.

Minvielle-Sebastia, L., B. Winsor, N. Bonneaud, and F. Lacroute. 1991. Mutations in the yeast RNA14 and RNA15 genes result in an abnormal mRNA decay rate; sequence analysis reveals an RNA-binding domain in the RNA15 protein. Mol Cell Biol 11: 3075-3087.

Miozzari, G., P. Niederberger, and R. Hutter. 1978. Tryptophan biosynthesis in Saccharomyces cerevisiae: control of the flux through the pathway. J Bacteriol 134: 48-59.

Mullaney, E.J., J.E. Hamer, K.A. Roberti, M.M. Yelton, and W.E. Timberlake. 1985. Primary structure of the trpC gene from Aspergillus nidulans. Mol Gen Genet 199: 37-45.

Paluh, J.L., M.J. Orbach, T.L. Legerton, and C. Yanofsky. 1988. The cross-pathway control gene of Neurospora crassa, $c p c-1$, encodes a protein similar to GCN4 of yeast and the DNA-binding domain of the oncogene v-jun-encoded protein. Proc Natl Acad Sci U S A 85: 3728-3732.

Parker, A.R., T.D. Moore, J.C. Edman, J.M. Schwab, and V.J. Davisson. 1994. Cloning, sequence analysis and expression of the gene encoding imidazole glycerol phosphate dehydratase in Cryptococcus neoformans. Gene 145: 135-138.

Piotrowska, M. 1980. Cross-pathway regulation of ornithine carbamoyltransferase synthesis in Aspergillus nidulans. J Gen Microbiol 116: 335-339.

Pringle, J.R., A.E. Adams, D.G. Drubin, and B.K. Haarer. 1991. Immuno-fluorescence methods for yeast. Methods Enzymol 194: 565-602.

Punt, P.J. and C.A. van den Hondel. 1992. Transformation of filamentous fungi based on hygromycin B and phleomycin resistance markers. Methods Enzymol 216: 447-457.

Sambrook, J., E. Fritsch, and T. Maniatis. 1989. Molecular Cloning: A Laboratory Manual. Cold Spring Harbor Laboratory Press, Cold Spring Harbor, New York.

Serlupi-Crescenzi, O., M.B. Kurtz, and S.P. Champe. 1983. Developmental defects resulting from arginine auxotrophy in Aspergillus nidulans. J Gen Microbiol 129: 3535-3544.

Struhl, K. 1982. Regulatory sites for HIS3 gene expression in yeast. Nature 300: 285-286.

Wanke, C., S. Eckert, G. Albrecht, W. van Hartingsveldt, P.J. Punt, C.A. van den Hondel, and G.H. Braus. 1997. The Aspergillus niger GCN4 homologue, $c p c A$, is transcriptionally regulated and encodes an unusual leucine zipper. Mol Microbiol 23: 23-33.

Winter, E. and A. Varshavsky. 1989. A DNA binding protein that recognises oligo(dA)-oligo(dT) tracts. EMBO J 8: 1867-1877. 


\section{Impact of the cross-pathway control on regulation of lysine and penicillin biosynthesis in Aspergillus nidulans.}

\subsection{Abstract}

The non-proteinogenic amino acid $\alpha$-aminoadipate defines the biosynthetic branch point of lysine and penicillin biosynthesis in the filamentous fungus Aspergillus nidulans. Regulation of both pathways was analysed in response to amino acid limitation. The lys $F$ encoded homoaconitase acts upstream of the $\alpha$-aminoadipate branch point whereas the lys $A$ gene product saccharopine dehydrogenase catalyses the ultimate step of the lysine-specific branch. The lysA gene from A. nidulans was identified and isolated. Amino acid starvation resulted in significantly increased transcription of lysA but not of lysF. Starvation dependent changes in transcription levels of lys $A$ were dependent on the presence of the central transcriptional activator of the cross-pathway control (CPCA). The effect of amino acid starvation under penicillin production conditions was analysed in A. nidulans strains with reporter genes for the penicillin biosynthesis genes acvA and ipnA and genetically altered activity of the cross-pathway control. Overproduction of CPCA decreased expression of ipnA and acvA reporter genes and even more drastically reduced penicillin production. This work suggests that, upon amino acid starvation, the cross-pathway control overrules secondary metabolite biosynthesis and favours the metabolic flux towards amino acids instead of penicillin in $A$. nidulans.

\subsection{Introduction}

Aspergillus nidulans serves as fungal model organism for regulatory networks in eukaryotes. The regulation circuits which direct metabolic fluxes towards either primary or secondary metabolism are scarcely known (Firn and Jones 2000). An immediate connection of primary- and secondary metabolism in $\beta$-lactam producing filamentous ascomycetes is represented by the $\alpha$-aminoadipate branch point of lysine and penicillin biosynthesis. Both metabolites are of biotechnological importance: lysine is an essential amino acid for humans which has to be supplied by the diet, and $\beta$-lactam antibiotics are among the most prominent antibiotics used world-wide. A. nidulans is due to its sexual cycle more suitable for genetic studies on regulation of penicillin biosynthesis than the commercial production strains of Penicillium chrysogenum (Martin 1998). The initial five enzymatic reactions starting from $\alpha$-ketoglutarate and acetylCoA result in the key intermediate $\alpha$-aminoadipate (Fig. 3.1). The biosynthesis branches at this point and leads in 


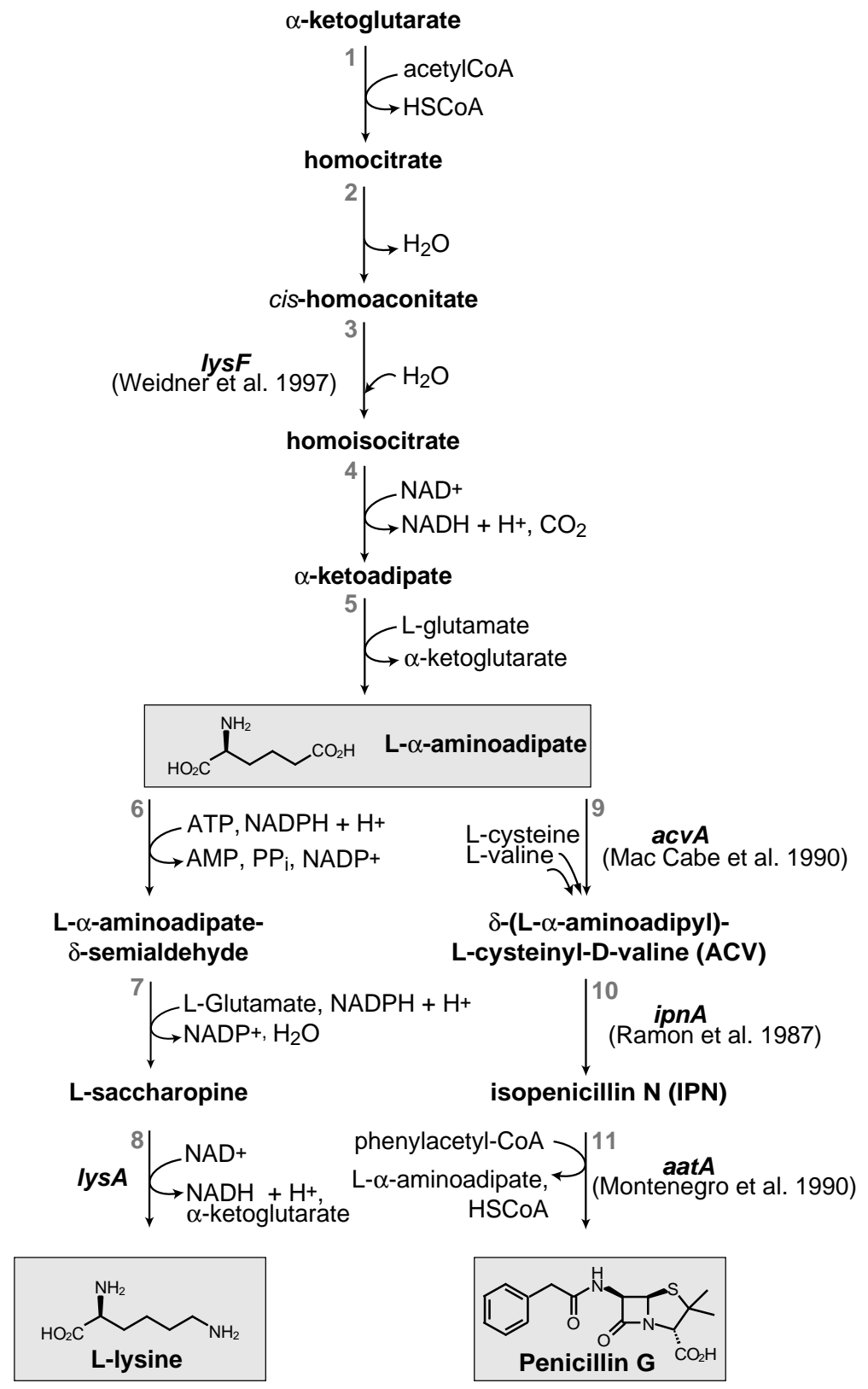

Fig. 3.1: $\alpha$-aminoadipate constitutes a branch point of lysine and penicillin biosynthesis in $\beta$-lactam antibiotic-producing fungi. The $\alpha$-aminoadipate pathway of lysine biosynthesis is common to fungi and includes eight enzymatic steps: (1) homocitrate synthase EC 4.1.3.21, $(2,3)$ homoaconitase EC 4.2.1.36, (4) homoisocitrate dehydrogenase EC 1.1.1.87, (5) aminoadipate aminotransferase EC 2.6.1.39, (6) aminoadipate reductase EC 1.2.1.31, (7) saccharopine reductase: EC 1.5.1.10 and (8) saccharopine dehydrogenase EC 1.5.1.7 (Zabriskie and Jackson 2000). Penicillin is synthesised via (9) ACV synthetase, (10) IPN synthase and (11) AcylCoA:IPN acyltransferase (Brakhage 1998). Genes isolated from $A$. nidulans encoding enzymes mediating any of the steps mentioned above are shown in bold print.

three enzymatic steps either towards lysine or penicillin (Ramon et al. 1987; Zabriskie and Jackson 2000). The regulatory circuits controlling the distribution of the key metabolite $\alpha$-aminoadipate between the two branches are yet unknown. Taking into account that lysine is needed for translation of proteins but the physiological role of the $\beta$-lactam antibiotic for the fungal life is yet unclear, the regulatory potential at this point is of special interest.

The lys $F$ gene is the only gene of the lysine/penicillin pathway's common stem which has been isolated and characterised from $A$. nidulans. It encodes 
homoaconitase LYSF which catalyses the third and presumably also the second step of lysine biosynthesis (Weidner et al. 1997). A negative effect on lys $F$ expression is mediated by external supply of lysine and the CCAAT-binding complex AnCF (Weidner et al. 1997; Steidl et al. 1999; Weidner et al. 2001). None of the genes involved in the conversion of $\alpha$-aminoadipate into L-lysine are yet isolated. By contrast, all genes involved in the penicillin-specific branch of A. nidulans are known. The amino acids $\mathrm{L}-\alpha$-aminoadipate, $\mathrm{L}$-cysteine and $\mathrm{L}$-valine are condensed by a non-ribosomal peptide synthetase (ACVS) resulting in the ACV-tripeptide $\delta$-(L- $\alpha$-aminoadipyl)-L-cysteinyl-D-valine (MacCabe et al. 1990). The subsequent oxidative ring closure to isopenicillin $\mathrm{N}$ is catalysed by the isopenicillin N synthase IPNS (Schenk 2000). The acylCoA: isopenicillin $\mathrm{N}$ acyltransferase (IAT) exchanges the $\alpha$-aminoadipate side chain with a phenylacetyl moiety resulting in the acylated dipeptide penicillin G (Whiteman et al. 1990). In all known penicillinproducing fungi, the penicillin biosynthesis genes are located in a single cluster with the genes acvA and ipnA being divergently transcribed from a bidirectional promoter region. Expression of the genes is controlled by complex regulatory processes depending on the developmental state of the culture and on growth conditions (Brakhage 1998; Martin 2000). Particularly inclusion of corn steep solids, a by-product of the corn wet-milling industry, to the penicillin fermentation broth significantly stimulates expression of the penicillin biosynthesis genes and increases penicillin production by yet unknown mechanisms (MacCabe et al. 1990; Luengo and Penalva 1994).

It is conceivable that a cross-talk exists between regulation of lysine and penicillin biosynthesis. In the bifurcated biosynthesis pathway, amino acids are end product and precursor of the different branches, respectively (Fig. 3.1). Availability of amino acids might thus affect the metabolic flux towards $\alpha$ aminoadipate, lysine and penicillin. For the genes acting downstream of $\alpha$ aminoadipate, there are two interesting alternatives which could be pursued by the fungus: penicillin synthesis could be either reduced to favour lysine biosynthesis or increased to kill food competitors as e.g. bacteria and use them as protein or nitrogen source. In A. nidulans and other filamentous fungi, limitation of amino acids activates the global regulatory network "cross-pathway control" (Sachs 1996). The transcriptional activator of this system, CPCA (Hoffmann et al. 2001), binds to CPCA response elements (CPREs) in the promoter region of the corresponding target genes and increases their transcription. The optimal binding site for Gcn $4 p$, the CPCA homologue in Saccharomyces cerevisiae, is the asymmetric sequence 5'-TGA(C/G)TCA-3', though Gcn4p can also bind with lower affinity to sequences that differ from this optimal sequence in one or even two positions (Hinnebusch 1986; Oliphant et al. 1989; Mavrothalassitis et al. 1990). In A. nidulans, the tryptophan biosynthesis gene trpC (Eckert et al. 2000) and the histidine genes hisB (Busch et al. 2001) and hisHF (Valerius et al. 2001) contain CPREs in their 5، regulatory region and are transcriptionally activated upon amino acid starvation. But another amino acid biosynthesis gene, aroC, is not affected by this system (Krappmann et al. 1999) whereas a gene probably not related to amino acid biosynthesis is (Strittmatter et al. 2001), indicating that regulation by the cross- 
pathway regulatory network in A. nidulans is not restricted to amino acid biosynthesis genes.

Lysine biosynthesis is highly regulated by Gcn4p in yeast (Natarajan et al. 2001). But to our knowledge, data on regulation of penicillin biosynthesis by amino acid starvation are limited. External supply of amino acids to $A$. nidulans liquid cultures results in various effects on penicillin reporter gene expression, probably independent of the cross-pathway control system (Brakhage and Turner 1992; Then Bergh and Brakhage 1998). In P. chrysogenum, artificially induced histidine starvation differentially affects the $\alpha$-aminoadipate pool size and penicillin production dependent on the strain used (Hönlinger et al. 1988). In this work, the implication of amino acid starvation and corresponding activation of the crosspathway control on selected genes acting in the different branches of the bifurcated lysine/penicillin biosynthesis pathway in A. nidulans was questioned. Two experimental set-ups were applied, artificially induced histidine starvation and genetically altered intracellular CPCA levels. The results of this work suggest that amino acid starvation favours the metabolic flux towards amino acids and diminishes the flux towards penicillin at the $\alpha$-aminoadipate branch point of A. nidulans.

\subsection{Experimental procedures}

\subsubsection{Growth conditions}

A. nidulans strains were cultivated at $30^{\circ} \mathrm{C}$ in $100 \mathrm{ml}$ liquid minimal medium (50 mM glucose, $70 \mathrm{mM} \mathrm{NaNO}, 7 \mathrm{mM} \mathrm{KCl}, 11.2 \mathrm{mM} \mathrm{KH}_{2} \mathrm{PO}_{4}$ (pH 5.5), $2 \mathrm{mM}$ $\mathrm{MgSO}_{4}, 1 \mathrm{x}$ trace elements) (Bennett and Lasure 1991) or fermentation medium (100 mM lactose, $2 \% \mathrm{w} / \mathrm{v}$ corn steep solids, $100 \mathrm{mM} \mathrm{CaCO}{ }_{3}, 50 \mathrm{mM} \mathrm{KH}_{2} \mathrm{PO}_{4}$, $3.3 \mathrm{mM}$ phenoxy acetic acid, pH 6.0) (Brakhage and Turner 1992) supplemented as described (Käfer 1977). For amino acid starvation, mycelia of liquid overnight precultures $\left(10^{9}\right.$ spores in $300 \mathrm{ml}$ medium) were filtrated through sterile miracloth, transferred into fresh minimal/fermentation medium and grown for $1 \mathrm{~h} / 40 \mathrm{~h}$ prior to addition of $10 \mathrm{mM} / 50 \mathrm{mM}$ 3-amino-1,2,4-triazole (3AT), respectively. Expression from the alcA promoter in the complex fermentation medium was induced by $10 \mathrm{mM}$ cyclopentanone as described before (Waring et al. 1989; Kennedy and Turner 1996).

\subsubsection{Isolation procedures}

Three independent cultures were used to extract RNAs, DNAs and proteins. A. nidulans mycelia were filtered through sterile miracloth, freeze-dried and mechanically pulverised. RNAs were isolated from a volume of $100 \mu \mathrm{l}$ mycelia mixed with $1 \mathrm{ml}$ Trizol $^{\mathrm{TM}}$ (GIBCO BRL) as recommended by the manufacturers. DNAs were isolated from a volume of $1 \mathrm{ml}$ mycelia according to Lee and Taylor (Lee and Taylor 1990). Crude protein extracts were obtained from the supernatant 
derived from $50 \mathrm{mg}$ lyophilised mycelia ground with equal volume of sterile sand and mixed with $3.5 \mathrm{ml}$ cold $0.2 \mathrm{M}$ phosphate buffer.

\subsubsection{Hybridisation experiments}

$50 \mu \mathrm{g}$ and $15 \mu \mathrm{g}$ of total RNA or $15 \mu \mathrm{g}$ chromosomal DNA per lane were separated in a 1.4\% formaldehyde-containing agarose gel or a $1 \%$ agarose gel, respectively. The RNA-ladder and $1 \mathrm{~kb}$-DNA-ladder of Gibco BRL were used as size standards. RNA quantities were standardised according to the 16S and 23S rRNA signals on the ethidium bromide stained gel and quantified via the Image Station 440CF (Kodak, Rochester, NY, USA). For Northern and Southern hybridisation experiments, nucleotides were transferred on nylon membranes by capillary blotting, fixed for $30 \mathrm{~min}$ at $65^{\circ} \mathrm{C}$ (GeneScreen) and crosslinked with UV light. For colony hybridisation experiments, cells were directly transferred from agar plates to the membrane and treated successively with $0.5 \mathrm{M} \mathrm{NaOH} / 1.5 \mathrm{M} \mathrm{NaCl}, 1.5 \mathrm{M}$ $\mathrm{NaCl} / 0.5 \mathrm{M}$ Tris ( $\mathrm{pH} 7.4$ ) and $2 \times$ SSC. Membranes were prehybridised for $2 \mathrm{~h}$ in hybridisation mix (7\% SDS, 1\% BSA, 1 mM EDTA, $250 \mathrm{mM} \mathrm{NaPO}_{4}$, pH 7.2). Specific probes for acvA, aro $C, c p c A, g p d A$, hisB, ipnA, lys $A$ and $l y s F$ were amplified via PCR with T3 and T7 primers or specific primer pairs (Tab. 3.1) and ${ }^{32} \mathrm{P}$-labelled with the HexaLabel ${ }^{\mathrm{TM}}$ DNA Labelling Kit (MBI Fermentas). Following overnight hybridisation at $65^{\circ} \mathrm{C}$, membranes were rinsed twice and washed for 30 min with $0.1 \times$ SSC / 0.1\% SDS, dried on air and exposed to imaging plates and x-ray films. Signal intensities were quantified using a bioimager and MacBAS v2.5(E) from Fuji.

\subsubsection{Enzymatic assays}

$100 \mu \mathrm{l}$ of protein extracts were mixed with $0.9 \mathrm{ml}$ Z-buffer (48 $\mathrm{mM} \mathrm{Na}_{2} \mathrm{HPO}_{4}$, $39.3 \mathrm{mM} \mathrm{NaH} \mathrm{PO}_{4}, \quad 10 \mathrm{mM} \mathrm{KCl} \mathrm{pH} 7.0, \quad 0.1 \mathrm{mM} \mathrm{MgSO}_{4}, \quad 0.01 \mathrm{mM}$ PMSF, $270 \mu \mathrm{l} / 100 \mathrm{ml} \beta$-mercaptoethanol) and adapted to $25^{\circ} \mathrm{C}$ in a waterbath. For the photometric $\beta$-galactosidase assay, the reaction was started with $200 \mu$ l ONPGsolution (4 mg o-nitrophenol- $\beta$-D-galactoside / $\mathrm{ml}$ Z-buffer) and stopped after 1 to 30 min with $500 \mu \mathrm{l} 1 \mathrm{M} \mathrm{Na}_{2} \mathrm{CO}_{3}$-solution. Absorbance was measured at $420 \mathrm{~nm}$ and specific activity determined as $(\mathrm{U} / \mathrm{mg})=1.7 \times \mathrm{OD}_{420} /$ extinction coefficient $\mathrm{x}$ protein concentration $x$ extraction volume $x$ time. The photometric $\beta$-glucuronidase assay was started with PNPG (4 mg/ml p-nitrophenol- $\beta$-D-glucuronic acid), stopped with $500 \mu \mathrm{l} 1 \mathrm{M} \mathrm{Na}_{2} \mathrm{CO}_{3}$-solution and absorbance was measured at $415 \mathrm{~nm}$. Protein content was determined via the Bradford assay (Bradford 1976).

\subsubsection{Penicillin bioassay}

$1 \mathrm{ml}$ samples of $A$. nidulans liquid culture supernatants (stored at $-20^{\circ} \mathrm{C}$ ) were centrifuged at $16000 \mathrm{~g}$ for $30 \mathrm{~min}, 50,25$ and $10 \mu \mathrm{l}$ of the supernatant were dropped on sterile antibiotic test filters $(\varnothing 9 \mathrm{~mm}$, Schleicher and Schüll) and dried. Test filters and calibration samples (50 to $0.003 \mu \mathrm{g}$ penicillin $\mathrm{G} / \mathrm{ml}$ ) were placed on $\mathrm{M} 1$ medium agar plates $(0.5 \% \mathrm{w} / \mathrm{v}$ peptone, $0.3 \% \mathrm{w} / \mathrm{v}$ meat extract, $1.5 \% \mathrm{w} / \mathrm{v}$ agar) confluently covered with overnight colonies of the penicillin-sensitive strain 
Bacillus calidolactis $\mathrm{C} 953$ and incubated at $55^{\circ} \mathrm{C}$ overnight. The penicillin titre was determined according to the bacterial growth as log concentration versus retardation ring in $\mathrm{mm}$.

\subsubsection{Plasmid construction}

Plasmids and specific primer pairs used in this study are summarised in Table 3.1. The nucleotide sequence of the $S$. cerevisiae LYS1 gene was used for identification of a $434 \mathrm{bp}$ fragment in the $A$. nidulans EST library via homology searches. This lysA fragment was amplified via PCR and subcloned into pBluescript ${ }^{\circledR} I I S K+($ Stratagene) resulting in plasmid pME1750. The insert was used as specific probe in a colony hybridisation experiment of a partially Sau3A digested A. nidulans genome library (obtained from Bruce Miller, Moskow, Idaho, USA) yielding plasmid pME2159 with a $14 \mathrm{~kb}$ insert. From this insert, an eight kb Kpnl fragment containing the complete lysA coding region was subcloned into pBluescript ${ }^{\circledR} I I S K+$ resulting in plasmid pME2162.

Tab. 3.1: Plasmids

\begin{tabular}{|c|c|c|}
\hline plasmid & insert description (primer for PCR amplification) & reference \\
\hline pAN5-22 & $\begin{array}{l}1.2 \mathrm{~kb} \text { genomic } A \text {. nidulans BamHl/EcoRl-insert } \\
\text { containing gpdA in pBR322 }\end{array}$ & Punt et al. 1988 \\
\hline pBluescript II & $2.96 \mathrm{~kb}$ cloning vector (pBSK and pBKS) & Stratagene, CA, USA \\
\hline pLYSF1 & $\begin{array}{l}3.1 \mathrm{~kb} \text { genomic } A . \text { nidulans Hindlll } / S p h \text {-insert } \\
\text { containing lys } F \text { in pUC18 }\end{array}$ & Weidner et al. 1997 \\
\hline pME1498 & $1 \mathrm{~kb}$ aro $C$ cDNA of $A$. nidulans Sall/Notl in pBKS & Krappmann et al. 1999 \\
\hline pME1511 & $1 \mathrm{~kb}$ hisB cDNA of $A$. nidulans Sall/Notl in pRS316-GAL1 & Busch et al. 2001 \\
\hline pME1603 & $\begin{array}{l}c p c A \text { open reading frame fused to the alcA-promoter } \\
\left(\mathrm{P}_{\text {alcA}}-C P C A\right) \text { in } \mathrm{pME} 1565\end{array}$ & Hoffmann et al. 2001 \\
\hline pME1702 & $\begin{array}{l}2.2 \mathrm{~kb} c p c A \text { cDNA of } A \text {. nidulans Sall/Notl in pRS316- } \\
\text { GAL1 }\end{array}$ & Hoffmann et al. 2001 \\
\hline pME1707 & $\begin{array}{l}2.7 \mathrm{~kb} \text { genomic } A \text {. nidulans } X \text { bal/BamHI-insert } \\
\text { containing } c p c A \text { in pRG3 }\end{array}$ & Hoffmann et al. 2001 \\
\hline pME1750 & $\begin{array}{l}434 \text { bp lys } A \text { insert in pBSK } \\
\text { (5'-taatggactccaagctcatg-3', 5'-tctcttcagatcttaccagc-3') }\end{array}$ & this work \\
\hline pME1751 & $\begin{array}{l}501 \text { bp acvA insert in pBSK } \\
\left(5^{`} \text {-gatttaaagaccacctac-3', } 5^{`} \text {-ctcccccaattcgatacg-3`) }\right.\end{array}$ & this work \\
\hline pME1752 & $\begin{array}{l}503 \text { bp ipnA insert in pBSK } \\
\left(5^{`} \text {-caggaccaagtccgtgcc-3‘, } 5^{\star} \text {-caagtagccagtattcgtc-3`) }\right.\end{array}$ & this work \\
\hline pME2159 & $14 \mathrm{~kb}$ genomic $A$. nidulans insert containing $l y s A$ in pUl1 & this work \\
\hline pME2162 & $\begin{array}{l}8 \mathrm{~kb} \text { genomic } A \text {. nidulans Kpnl-insert } \\
\text { containing lys } A \text { in pBSK }\end{array}$ & this work \\
\hline
\end{tabular}

\subsubsection{Sequence analyses}

For DNA sequence analysis with custom oligonucleotides (Gibco BRL) the Big Dye $^{\mathrm{TM}}$ Terminator kit of the Cycle Sequencing Reaction Mix (Perkin Elmer) was used. Sequence analysis was performed on a Perkin Elmer Sequenator 310 with the $A B I$ EditView 1.0.1 software. Further DNA analysis was performed with the Lasergene software from DNASTAR. Saccharopine dehydrogenase peptide sequences were identified using BLAST search of a non-redundant protein 
database (Altschul et al. 1997). Pairwise alignments and the multiple sequence alignment with hierarchial clustering was performed using the software "CLUSTALW" (Corpet 1988). The nucleotide sequence of the lys $A$ coding region has been deposited in the GenBank database under GenBank Accession Number AY057447.

\subsubsection{A. nidulans strain construction}

Isogenic $A$. nidulans strains containing acvA and ipnA reporter gene fusions and different expression levels of $c p c A$ were constructed by crossing experiments. Genotypes and sources of the $A$. nidulans strains are given in Table 3.2. The $c p c A$ deletion $(\triangle c p c A:: b l e o)$ of strain AGB52 was combined with the mutated $\beta$ galactosidase allele (bga0) of strain WG355 resulting in strain AGB178. The bga0phenotype of $A$. nidulans strains was tested in a $\beta$-galactosidase assay on minimal medium agar plates containing either glucose or lactose as carbon source. Cells were permeabilised with toluol, washed and incubated with 4-methylumbelliferyl- $\beta$ D-galactosid $(4 \mathrm{mg} / \mathrm{ml}$ ) to visualise the fluorescence at $320 \mathrm{~nm}$. The reporter gene fusions of the penicillin biosynthesis genes (argB2::PacvA-uidA; PipnA-lacZ; $\arg B^{+}$) from strain $A X B 4 A$ were crossed into strain $A G B 178$. The resulting $c p c A$ deletion strain AGB187 showed $\beta$-galactosidase activities solely expressed by the reporter genes. AGB187 was transformed with a wild-type copy of $c p c A$ (plasmid pME1707) or a $c p c A$ overproduction construct (plasmid pME1603) resulting in strains AGB188 (single integration of $c p c A$ ) and AGB189 (5-6 copies of PalcA-cpcA ectopically integrated at two genome loci). Genotypes were tested by PCR and Southern hybridisation analyses.

Tab. 3.2: A. nidulans strains

\begin{tabular}{|c|c|c|}
\hline strain & genotype & reference \\
\hline WG355 & $\operatorname{argB2} ; b g A 0 ; b i A 1$ & Brakhage et al. 1992 \\
\hline A234 & yA2, pabaA1; veA1 & FGSC ${ }^{\star}$ \\
\hline AXB4A & bga0; biA1; $\arg B 2:: \mathrm{P}_{a c v A}-u i d A \mathrm{P}_{\text {ipnA-lacZ, }}$ arg $B^{+}$ & Brakhage et al. 1992 \\
\hline AGB52 & $\triangle c p c A::$ bleo; pabaA1; pyrG89; pyroA4; veA1; yA2 & Hoffmann et al. 2001 \\
\hline AGB178 & bga0; $\triangle c p c A:: b l e o ; \operatorname{argB2;}$ pyrG89 & this work \\
\hline AGB187 & 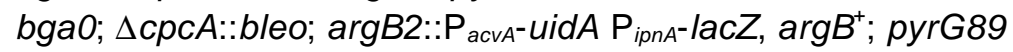 & this work \\
\hline AGB188 & 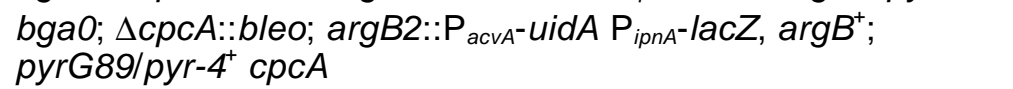 & this work \\
\hline AGB189 & 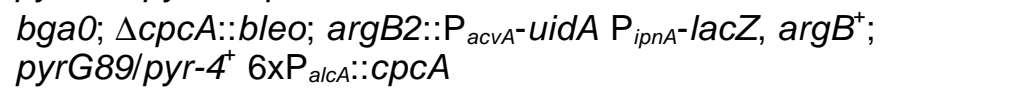 & this work \\
\hline
\end{tabular}

* Fungal Genetics Stock Center (University of Kansas Medical Center, Kansas City, KS, USA). 


\subsection{Results}

\subsubsection{The IysA gene of $A$. nidulans shows up to $62 \%$ amino acid indentities to lysine-forming saccharopine dehydrogenases}

The effect of amino acid starvation on the regulation of genes situated in the different branches of the bifurcated lysine/penicillin synthesis pathway was questioned. The only lysine biosynthesis gene isolated from $A$. nidulans to date encodes the homoaconitase LYSF acting upstream of the $\alpha$-aminoadipate branch point. The lys $F$ gene comprises an open reading frame of $2325 \mathrm{bp}$ interrupted by one intron with a transcript size of about $2.7 \mathrm{~kb}$ (Weidner et al. 1997). Since no gene of the lysine-specific branch of the pathway was isolated yet, a $7.5 \mathrm{~kb}$ genomic $K p n l$-fragment containing the complete $A$. nidulans lys $A$ locus was isolated from an $A$. nidulans genomic library by colony hybridisation with a lys $A$ specific probe. DNA sequencing revealed that the lys $A$ coding region spans $1673 \mathrm{bp}$ including eight putative introns of 47 to $171 \mathrm{bp}$ (Fig. 3.2A). Southern hybridisation experiments with genomic $A$. nidulans DNA digested with several restriction endonucleases suggested that lys $A$ is a single copy gene in this fungus (data not shown). From the deduced peptide sequence of 375 amino acids (aa) a relative mass of $41 \mathrm{kDa}$ was calculated for the gene product. The deduced polypeptide sequence showed high identities to $\mathrm{NAD}^{+}$-dependent, L-lysine forming saccharopine dehydrogenases (SDH; EC 1.5.1.7) of fungal origin, like from S. cerevisiae (Ogawa et al. 1980), Y. lipolytica (Xuan et al. 1990), C. albicans (Garrad et al. 1994) and S. pombe (Gentles et al. 1995) (Fig. 3.2B). The identities of up to $62 \%$ strongly suggest that the saccharopine dehydrogenase (LYSA) encoding gene from $A$. nidulans was identified.

\subsubsection{Transcript levels are increased for IysA but reduced for IysF by starvation-dependent activation of CPCA}

Implication of the cross-pathway control on lys $F$ transcription seemed conceivable since in silico analysis revealed one sequence within $250 \mathrm{bp}$ of the promoter region (5'-TGAGTCT-3') that differed in only one position from the optimal Gcn $4 p$ binding motif and could thus possibly serve as CPRE. lys $F$ transcript levels of mycelia grown under standard and amino acid limited conditions were compared (Fig. 3.3B). Amino acid starvation was induced by addition of the false feedback inhibitor 3-amino-1,2,4-triazole (3AT), which causes histidine starvation with subsequent induction of the cross-pathway control (Sachs 1996). Mycelia of wildtype strain A234 and the cpcA deletion strain AGB52 were cultivated in standard minimal medium with and without histidine limitation for 6 hours. Specific transcript levels in Northern hybridisation experiments were quantified and equalised to the rRNA signal intensities. The relative transcription level of the wild-type grown at standard conditions in minimal medium was set as one. As controls, transcription levels of the hisB (Busch et al. 2001) and aroC (Krappmann et al. 1999) amino acid biosynthesis genes and of gpdA were monitored (data not shown). When cells of 
A

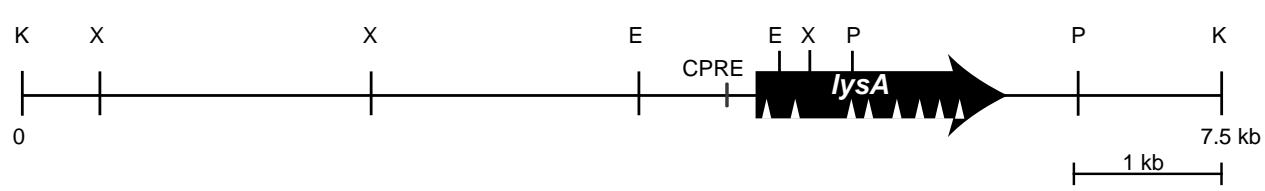

B

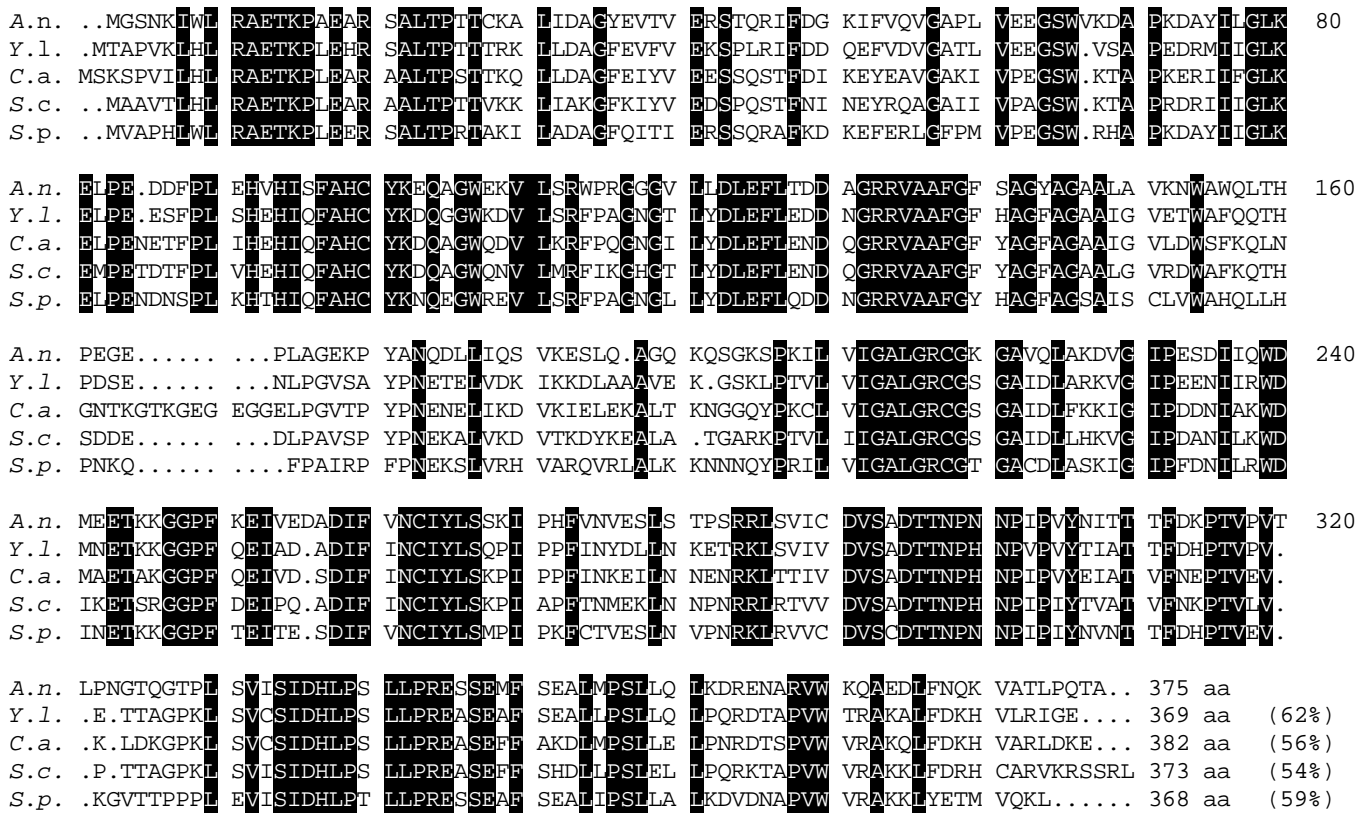

Fig. 3.2: The deduced peptide sequence of the A. nidulans lys $A$ gene shows up to $62 \%$ amino acid identities to other fungal saccharopine dehydrogenases. (A) Schematic overview of the lysA locus in A. nidulans. Plasmid pME2162 contains the complete lys $A$ coding region within a $7.5 \mathrm{~kb}$ genomic DNA fragment of $A$. nidulans. Introns are indicated by white triangles. CPRE indicates the proposed cross-pathway response element. Restriction sites of $K p n l(K), X b a l(X), E c o R I(E)$ and Pstl (P) are indicated. (B) The global multialignment of fungal saccharopine dehydratases of A. nidulans LYSA (A.n.; GenBank Accession Number AY057447), Yarrowinia lipolytica (Y.I.; P38997), Candida albicans (C.a.; P43065), Saccharomyces cerevisiae (S.c.; P38998) and Schizosaccharomyces pombe (S.p.; Q09694) shows a high conservation level with amino acid identities of $45 \%$. Identical amino acids are indicated by black boxes. The percentage of identity of each single protein sequence to the A. nidulans (A.n.) lysA gene product is given in parentheses.

the $c p c A$ wild-type strain $A 234$ were starved for histidine, $c p c A$ but not lysF mRNA levels were elevated (Fig. 3.3B). On the contrary, transcription of lys $F$ was nearly halved compared to non-starvation conditions in the wild-type but not in the $c p c A$ deletion strain. These data suggest that lys $F$ as representative of the common stem of the pathway is not activated by the cross-pathway control system in response to amino acid starvation. The Northern hybridisation analyses revealed a lys $A$ transcript size of about $2 \mathrm{~kb}$. In minimal medium, transcription of lys $A$ in the wild-type strain A234 is increased about threefold upon amino acid starvation when compared to non-starvation (Fig. 3.3B). Correspondingly, one potential CPRE perfectly matching the Gcn4p consensus motif was identified in the lysA promoter region (5'-TGACTCA-3') with the central $C$ at position -174 . The increase in lys $A$ transcription levels during amino acid limitation seems to be primarily dependent on the transcription factor CPCA, because the cpcA deletion strain AGB52 is unable 
to elevate lysA mRNA levels in response to this stress situation (Fig. 3.2B). Thus, lys $A$ is a target gene of the cross-pathway control in $A$. nidulans.

\subsubsection{Steady state levels of lysF, lysA and $c p c A$ mRNA are reduced under penicillin production conditions}

The two penicillin-specific genes acvA (MacCabe et al. 1990) and ipnA (Ramon et al. 1987) are divergently transcribed from a bidirectional promoter region (Fig. 3.3A). Significant expression of these genes and corresponding penicillin production require an appropriate lactose-based complex medium including corn steep solids and a prolonged incubation period of about 48 hours. Mycelia grown in the standard minimal medium with glucose as carbon and $\mathrm{NaNO}_{3}$ as sole nitrogen source hardly express the penicillin biosynthesis genes (MacCabe et al. 1990; Then Bergh and Brakhage 1998). For transcriptional analyses, the $c p c A$ wild-type strain $A$. nidulans AXB4A that contains reporter gene fusions to the acvA-ipnA intergenic region (Fig. 3.4A) was used to allow subsequent analysis of the corresponding penicillin gene expression from the same mycelia batch. In addition to growth under standard minimal conditions, the strain was cultivated under penicillin production conditions in fermentation medium for 48 hours. Transcript levels of specific lysF, lysA and ipnA mRNAs from mycelia derived from fermentation medium and minimal medium, respectively, were compared (Fig. 3.3C). The cultures grown in penicillin production broth showed significantly lower levels of $l y s A$ and $l y s F$ mRNAs than cultures grown in minimal medium. Transcript levels were nearly halved for $l y s A$ and hardly detectable for lysF. The

Fig. 3.3: Transcriptional regulation of $I y s F$, IysA and ipnA in response to amino acid supply and functionality of the cross-pathway control. The transcriptional level of specific mRNAs was analysed in Northern hybridisation experiments with RNA isolated from three independent cultures each. Mean signal intensities were equalised with respect to rRNA levels due to the lack of an established internal reference gene constitutively transcribed at all conditions used in this study. Signal intensities were set as one for the $c p c A$ wild type strains. The values given in parentheses indicate the relative change in transcriptional levels between the corresponding conditions. The mean standard deviation did not exceed 20\%. Growth conditions: Mycelia of overnight cultures were transferred into fresh medium and then grown for $3 \mathrm{~h}$ or $40 \mathrm{~h}$ in minimal or fermentation medium prior to addition of $10 \mathrm{mM}$ or $50 \mathrm{mM} 3$-aminotriazole, respectively. Mycelia were harvested $8 \mathrm{~h}$ later so total growth time was $11 \mathrm{~h}$ or $48 \mathrm{~h}$ in minimal or fermentation medium (unless stated otherwise). Abbreviations: 3-aminotriazole (3AT), below detection limit (bd), PalcA-induction in fermentation medium (FMi), fermentation medium (FM), minimal medium (MM), phleomycin resistance marker (ble), promoter (P), transcript size (ts), upstream open reading frame (uORF). (A) The transcriptional units of lysA, lys $F$ (Weidner et al. 1997), acvA (MacCabe et al. 1990), ipnA (Perez-Esteban et al. 1993) and cpcA (Hoffmann et al. 2001) as well as the different $c p c A$ constructs are shown. Transcription sizes are given in parentheses. (B) Comparison of lysA and lys $F$ transcript levels at 3AT-induced amino acid starvation (after $6 \mathrm{~h}$ ) in the wild-type strain A234 and the $C P C A$ deletion strain AGB52. (C) Comparison of $3 A T$-induced amino acid starvation on transcriptional levels in minimal and fermentation medium in strain AXB4A which is wild-type in respect to $c p c A$. (D) Comparison of transcriptional levels in isogenic $A$. nidulans strains with different internal levels of CPCA. Different transcript sizes of $c p c A$ in the overproduction strain result from transcription controlled by the alcA promoter. 

A lysA genomic locus:
lysF genomic locus:
PlysA lysA (ts $2.0 \mathrm{~kb})$
PlysF $\quad$ lys $F($ ts $2.7 \mathrm{~kb})$
acvA / ipnA genomic locus:

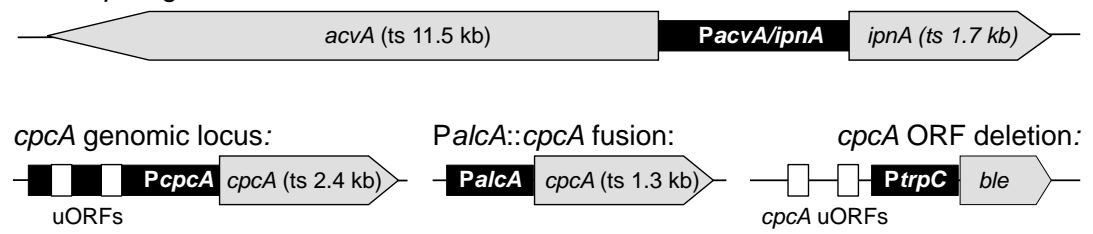

B
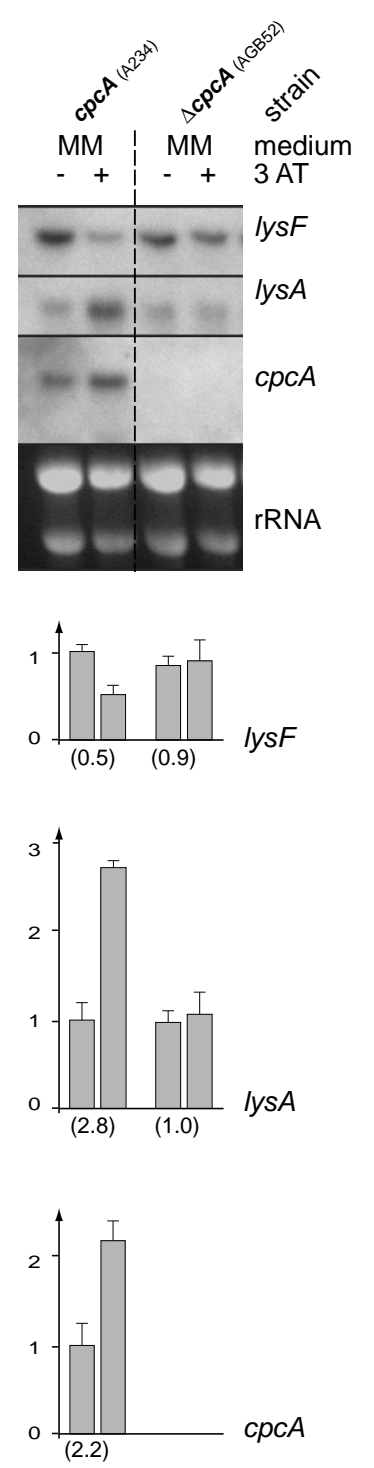

C
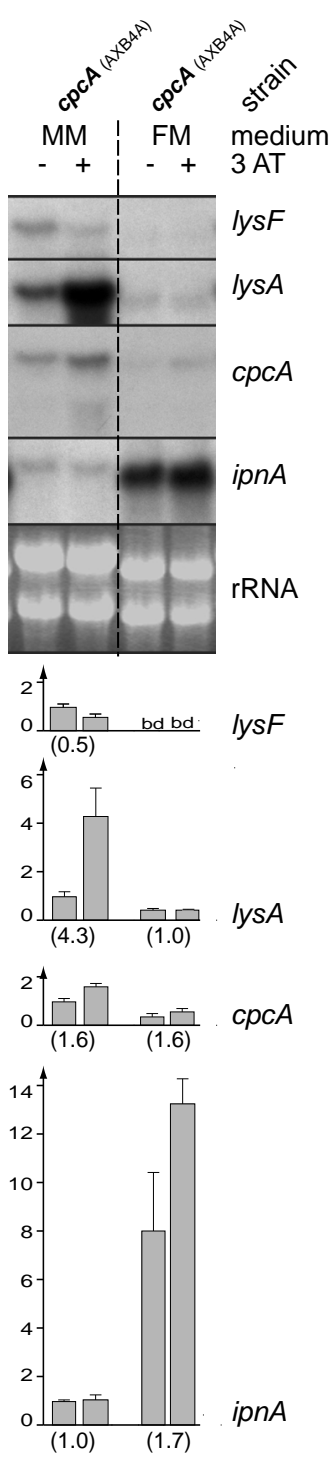

D
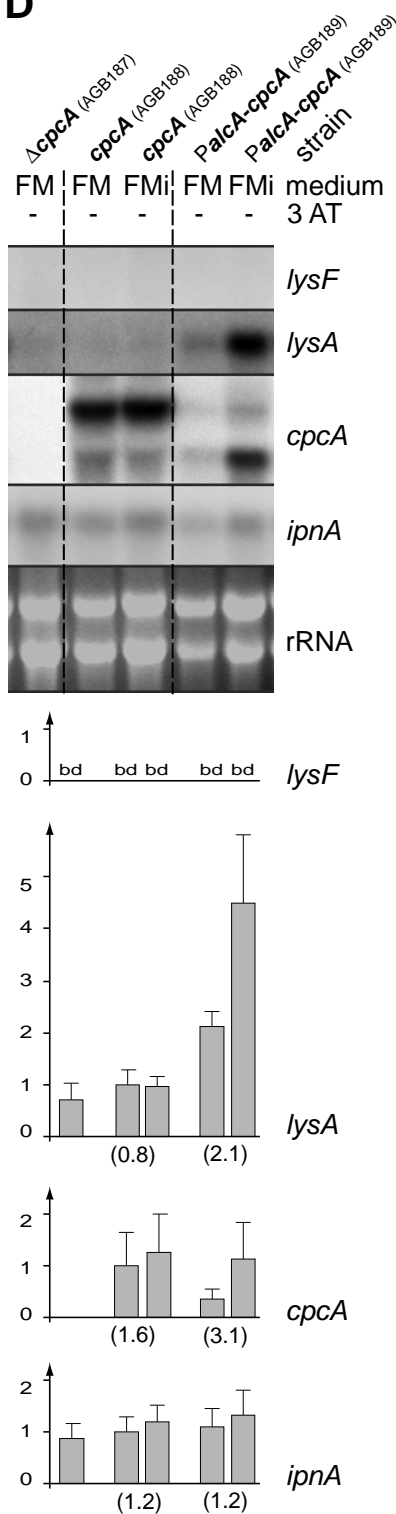

amount of $c p c A$ transcript is reduced in fermentation medium by about $30 \%$. As expected, transcription of $i p n A$, representing one of the two genes for the initial steps in penicillin biosynthesis, is approximately eight times higher in fermentation than in minimal medium.

To study the effect of amino acid starvation on penicillin biosynthesis under penicillin production conditions, the concentration of 3AT which induced histidine 
starvation in the complex fermentation medium was defined by determining increased transcription of $c p c A$, encoding the cross-pathway regulator. At $50 \mathrm{mM}$ $3 A T$ an 1.5 fold increase of $c p c A$ mRNAs was measured which corresponds to the 1.5 fold increase of the same transcript in minimal medium supplemented with $10 \mathrm{mM}$ 3AT. The $c p c A$ wild-type strain AXB4A was cultivated in fermentation medium for 40 hours and further incubated for 8 hours upon $3 A T$-induced histidine depletion. However, amino acid limitation monitored by $c p c A$ mRNAs increased Iys $A$ transcription in minimal medium fourfold whereas transcription of lys $A$ is about equal in fermentation medium regardless of histidine availability (Fig. 3.3C). ipnA mRNAs were significantly elevated by $3 A T$-addition in fermentation but not in minimal medium. These data suggest that in fermentation medium, a more careful analysis of the impact of the cross-pathway control is required with various defined levels of CPCA protein within the cell.

\subsubsection{CPCA overproduction decreases expression of the penicillin-specific genes as well as the penicillin titre}

To further analyse gene expression of the two penicillin biosynthesis genes, activities of reporter gene fusions of the divergent promoter region of ipnA and acvA (Fig. 3.4A) in A. nidulans strain AXB4A were measured. Crude protein extracts of mycelia grown in minimal and fermentation media with and without 3 AT-addition were isolated for quantification of specific reporter gene activities. In agreement with the results of the Northern hybridisation experiments, ipnA reporter gene expression was threefold higher in fermentation than in minimal medium (Fig. 3.4B). Expression of acvA was even increased by a factor of eight upon penicillin production conditions. Whereas the Northern hybridisation results suggested that 3AT-addition in fermentation medium slightly increased ipnA expression, both penicillin biosynthesis genes fused to reporter constructs showed even slightly reduced activities under these conditions. It remains to be elucidated whether an additional control mechanism besides transcriptional regulation is acting under these circumstances.

3AT-induced histidine starvation results in increased expression of the central transcription factor of the cross-pathway regulatory network, CPCA (Hoffmann et al. 2001). Isogenic A. nidulans strains were constructed that contain the penicillin reporter genes PacvA-uidA, PipnA-lacZ (AGB188) as well as deletion (AGB187) or overexpression (AGB189) of the cross-pathway transcription factor. In AGB189, $c p c A$ was fused to the alcA promoter (PalcA-cpcA) which allowed to modulate CPCA expression independently of the presence or absence of amino acids. PalcA is a well characterised filamentous fungal promoter (Waring et al. 1989; Mathieu and Felenbok 1994; Felenbok et al. 2001), and an expression system had been established for its use in the complex $A$. nidulans penicillin production broth. Without rendering the fermentation medium composition, this promoter shows only modest activity and can be induced by $10 \mathrm{mM}$ cyclopentanone (Kennedy and Turner 1996). In Northern hybridisation experiments (Fig. 3.2D), Iys A as a CPCA target gene was used to monitor the activity of the cross-pathway control 
A

acvA and ipnA reporter gene fusions:

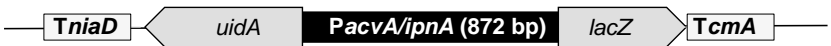

B
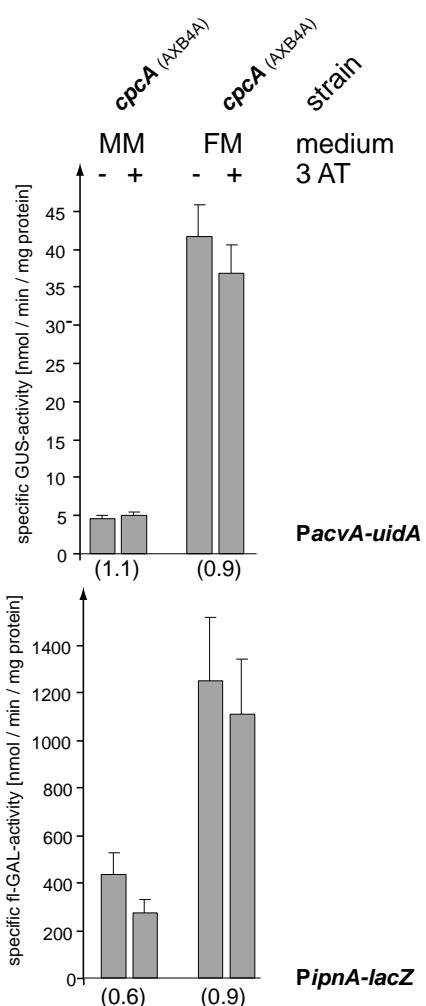

D

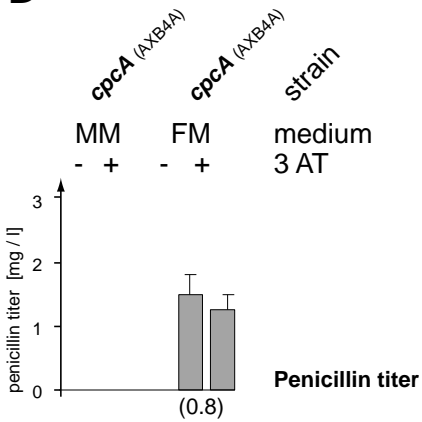

C
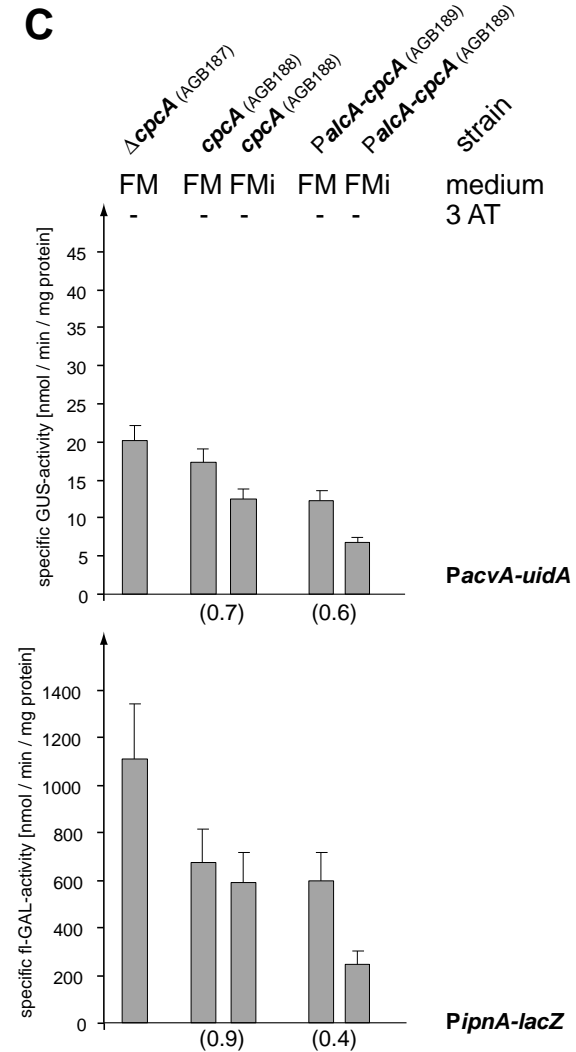

E
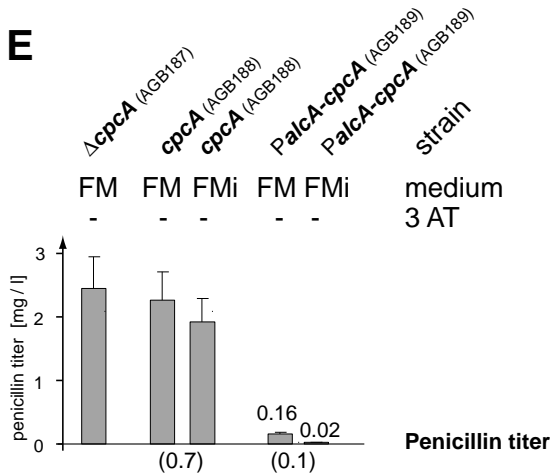

Fig. 3.4: Expression of the penicillin biosynthesis genes and the penicillin titre are decreased at high internal CPCA levels. The specific activity of $\beta$-glucuronidase (GUS) and $\beta$-galactosidase ( $\beta$-GAL) as well as the penicillin titre are shown. The values given in parentheses indicate the relative change in reporter gene expression or penicillin production levels between the corresponding conditions. The mean deviation did not exceed $21 \%$. See legend to Fig. 3.2 for growth conditions and abbreviations. (A) A. nidulans strain AXB4A and the isogenic strains AGB187, AGB188 and AGB189 contain reporter gene fusion for the penicillin genes acvA (PacvA-uidA) and ipnA (PipnA-lacZ) integrated at the argB locus (Brakhage et al. 1992). (B) Specific activities of the reporter genes are shown with respect to 3AT-induced amino acid starvation (C) and internal CPCA level. Additionally, the supernatant of the cultures was tested in a penicillin bioassay for antibiotic activity against the penicillin sensitive strain $B$. stearothermophilus (D) with respect to 3AT-induced amino acid starvation (E) and internal CPCA level.

since $c p c A$ expression is subject to strong post-transcriptional regulation (Hoffmann et al. 2001), The cpcA deletion strain AGB187 showed lowest lys $A$ mRNA levels and no $c p c A$ transcript (Fig. 3.3D). Strain AGB188 showed low levels 
of $c p c A$ transcript corresponding to the single wild-type copy and slightly increased lysA mRNA levels. Strain AGB189 exhibited low PalcA-driven $c p c A$ mRNA levels but threefold increased $c p c A$ transcript levels when the alcA promoter was induced. Accordingly, IysA transcript levels were increased. Thus, the strains AGB187, AGB188, AGB189 and AGB189 grown under cyclopentanone-induced conditions show increasing activity of the cross-pathway control which becomes prominent in successively increased transcription of lysA (Fig. 3.3D). The specific ipnA mRNA levels are hardly affected by changes in the CPCA concentration of the cell suggesting that the cross-pathway control does not regulate this penicillin biosynthesis gene on transcriptional level. In contrast to the mRNA levels, expression of the ipnA-lacZ reporter gene varied dependent on CPCA in the cell. The highest expression was found in the $c p c A$ deletion strain AGB187 (Fig. 3.4C). Overexpression of CPCA reduced ipnA-lacZ activity by more than twofold in AGB189. For the other penicillin biosynthesis gene, acvA, a similar CPCA dependent expression pattern was found when measured as activity of an acvAuid $A$ gene fusion.

The discrepancies between ipnA transcript levels which were even slightly increased in penicillin producing fermentation medium during 3AT-induced amino acid starvation and ipnA-lac $Z$ expression which was reduced when CPCA was overexpressed, prompted us to analyse the penicillin titre under various conditions. In minimal medium, penicillin production is repressed by glucose whereas the penicillin titre in fermentation medium is significant (Brakhage et al. 1992) and only slightly reduced when 3AT is added (Fig. 3.4D). Increased internal CPCA levels resulted in significantly decreased penicillin production (Fig. 3.4E). The CPCA overproduction strain AGB189 produces an order of magnitude less penicillin in comparison to $A$. nidulans strains with low or no CPCA levels. Though we cannot rule out side effects of the PalcA:cpcA ectopic integration in strain AGB189, the significant decrease of penicillin production upon PalcA:cpcA induction compared to non-induction counts for a real effect. Therefore, these data demonstrate that histidine starvation and accordingly an activated cross-pathway control increased transcription of $l y s A$, a lysine biosynthesis gene acting downstream of the $\alpha$ aminoadipate branch point, but does not activate the penicillin specific genes which channel this intermediate towards antibiotic biosynthesis. On the contrary, an even reduced expression of the penicillin biosynthesis genes and an even lower penicillin titre was found.

\subsection{Discussion}

After sharing a common pathway, lysine and penicillin biosyntheses separate at $\alpha$ aminoadipate and therefore have to compete for this intermediate. This work addressed the question whether stress induced by amino acid starvation impacts the regulation of the two branches. In synthetic $A$. nidulans minimal medium, amino acid starvation and subsequent activation of the cross-pathway control can be artificially induced by addition of the amino acid analogue 3AT (Sachs 1996). 
Corresponding transcriptional analyses in this work revealed $I y s A$ as an activated target gene of the cross-pathway control, but not lysF and ipnA. The 5'-regulatory region of IysA contained one $7 \mathrm{bp}$ sequence that perfectly matched the Gcn4p target motif (Oliphant et al. 1989; Mavrothalassitis et al. 1990). By contrast, in the promoter region of lys $F$ and in the acvA-ipnA intergenic region only nucleotide stretches differing with 1 or 2 bases from the consensus were identified. These findings indicate a direct regulation of lysA transcription by CPCA in response to amino acid starvation and suggest that at least the lysine-specific branch of the pathway is part of the cross-pathway network. Thus, elevated lysA transcription levels seem necessary to provide lysine when amino acids are limited. Similar results were obtained for $P$. chrysogenum, where the last two steps of the lysinespecific branch are subjected to the cross-pathway control, and $\alpha$-aminoadipate reductase is additionally included in this regulatory process in high production strains (Hönlinger et al. 1988).

It was shown here that artificial induction of the cross-pathway control by 3AT in penicillin production broth caused several side effects and thus this method seems restricted for use in fermentation medium. In this complex medium, corn steep solids mediate increased expression of the penicillin specific genes and finally a higher penicillin titre (MacCabe et al. 1990). Transcription levels of lys $A$ and, astonishingly, also of lys $F$ are significantly reduced in this medium. Thus, penicillin production seems not dependent on elevated lys $F$ transcription, though its gene product is involved in the biosynthesis of $\alpha$-aminoadipate. There are several possible explanations for this. Since the final reaction of penicillin biosynthesis recycles $\alpha$-aminoadipate, this compound might be re-utilized for another round of penicillin biosynthesis. Alternatively, lysine might be degraded to $\alpha$-aminoadipate to refill the pool required for the antibiotic biosynthesis. The $\alpha$ aminoadipate pool size which is critical for the rate of penicillin formation in P. chrysogenum (Jaklitsch et al. 1986; Hönlinger and Kubicek 1989; Lu et al. 1992) might be less important for A. nidulans penicillin biosynthesis (Brakhage and Turner 1992). Consistent with our findings for lys $F$ regulation in A. nidulans, expression of the first gene of the pathway's common stem in $P$. chrysogenum is reduced during the penicillin production phase. Additionally, overexpression of the corresponding gene did neither result in an increased $\alpha$-aminoadipate pool nor in higher penicillin production though homocitrate synthase is thought to be the ratelimiting step (Banuelos et al. 1999; Banuelos et al. 2000).

This work revealed a significantly lowered transcriptional level of $c p c A$ in the penicillin production medium. The effect of the cross-pathway transcriptional activator in complex broth was analysed in $A$. nidulans strains with genetically altered $c p c A$ expression where the activity of the cross-pathway control can be easily monitored by the transcriptional level of the target gene lysA. Significantly, a change in lysA transcriptional levels by factor two is accompanied with a decrease of the penicillin titre by about an order of magnitude. In contrast, mRNA levels of ipnA remain fairly constant irrespective of the cross-pathway activity. The corresponding ipnA reporter gene activities show a significant increase at highest cross-pathway activity only. These results suggest that there are differential effects 
of an activated cross-pathway system on penicillin biosynthesis in the penicillin production broth. Since transcription and reporter gene activity of penicillin genes and the corresponding penicillin titre do not correlate, these effects seem primarily mediated by post-transcriptional and post-translational events. Such effects have also been suggested for other aspects concerning regulation of $\beta$-lactam biosynthesis in filamentous fungi where discrepancies between gene expression and corresponding enzyme activity were observed (Brakhage 1998).

The reducing effects of an activated cross-pathway control on penicillin production could be mediated by different ways. Assuming that this effect is not due to a problem in penicillin export, two major explanations are conceivable. (I) CPCA probably increases lysine biosynthesis which could result in shortage of the $\alpha$ aminoadipate pool available for penicillin biosynthesis which cannot be compensated by the activation of $\alpha$-aminoadipate biosynthetic genes as lys $F$. This hypothesis is in agreement with findings in $P$. chrysogenum where high penicillin producing strains show lower turnover from $\alpha$-aminoadipate to lysine than low producing strains (Jaklitsch et al. 1986; Hönlinger and Kubicek 1989). Additionally, deletion of the aminoadipate reductase encoding gene, acting directly downstream of the $\alpha$-aminoadipate branch point, doubles the penicillin titre via the increased $\alpha$-aminoadipate pool (Casqueiro et al. 1999). On the basis of a doubled transcription rate of $I y s A$ at $c p c A$ overexpression, and assuming that the whole lysine specific branch downstream of the $\alpha$-aminoadipate branch point might be increased, the reduction of the penicillin titre might be due to the reduction of the $\alpha$ aminoadipate pool. Though in contrast to $P$. chrysogenum, the significance of the intermediate's pool size is not yet known since external supply of $\alpha$-aminoadipate cannot compensate the repressing effect of lysine on penicillin biosynthesis in A. nidulans (Brakhage and Turner 1992). However, this might be due to poor uptake of external $\alpha$-aminoadipate by A. nidulans (Brakhage 1998). (II) The expression of CPCA could mediate an indirect effect on penicillin biosynthesis genes by interfering with other regulatory proteins. It seems possible that CPCA activates another regulator which in turn acts negatively on penicillin biosynthesis enzymes. In yeast it was recently shown that 3AT-induced amino acid starvation which acts through the CPCA homologue Gcn $4 p$ modulates the activity of at least 26 different transcription factors and 11 protein kinases including regulators of amino acid biosynthesis, nitrogen utilisation and TCA cycle intermediates (Natarajan et al. 2001). It was shown that 3AT-induced starvation and CPCA overexpression also impacts the sexual development of $A$. nidulans, indicating a complex range of action of CPCA not only restricted to amino acid metabolism (Eckert et al. 2000; Busch et al. 2001). It is thus likely that CPCA does not directly act on the penicillin biosynthesis genes but on a yet unknown mediator.

In summary, a cross-talk was identified between the regulation of primaryand secondary metabolism mediated at least partly by the cross-pathway control. CPCA does not increase the penicillin biosynthesis pathway of A. nidulans. Moreover, the results suggest that under conditions resulting in an activated crosspathway control, penicillin synthesis is reduced and the lysine specific branch is favoured over the penicillin specific branch at the $\alpha$-aminoadipate branch point. 


\subsection{References}

Altschul, S.F., T.L. Madden, A.A. Schaffer, J. Zhang, Z. Zhang, W. Miller, and D.J. Lipman. 1997. Gapped BLAST and PSI-BLAST: a new generation of protein database search programs. Nucleic Acids Res 25: 3389-402.

Banuelos, O., J. Casqueiro, F. Fierro, M.J. Hijarrubia, S. Gutierrez, and J.F. Martin. 1999. Characterisation and lysine control of expression of the lys 1 gene of Penicillium chrysogenum encoding homocitrate synthase. Gene 226: 51-59.

Banuelos, O., J. Casqueiro, S. Gutierrez, and J.F. Martin. 2000. Overexpression of the lys1 gene in Penicillium chrysogenum: homocitrate synthase levels, alpha-aminoadipic acid pool and penicillin production. Appl Microbiol Biotechnol 54: 69-77.

Bennett, J.W. and L.L. Lasure. 1991. Growth media. In More gene manipulation in fungi (ed. J.W. Bennett and L.L. Lasure), pp. 441-457. Academic Press Inc, San Diego.

Bradford, M.M. 1976. A rapid and sensitive method for the quantitation of microgram quantities of protein utilizing the principle of protein-dye binding. Anal Biochem 72: 248-254.

Brakhage, A.A. 1998. Molecular regulation of $\beta$-lactam biosynthesis in filamentous fungi. Microbiol Mol Biol Rev 62: 547-585.

Brakhage, A.A., P. Browne, and G. Turner. 1992. Regulation of Aspergillus nidulans penicillin biosynthesis and penicillin biosynthesis genes acvA and ipnA by glucose. J Bacteriol 174: 3789-3799.

Brakhage, A.A. and G. Turner. 1992. L-lysine repression of penicillin biosynthesis and the expression of penicillin biosynthesis genes acvA and ipnA in Aspergillus nidulans. FEMS Microbiol Lett 77: 123127.

Busch, S., B. Hoffmann, O. Valerius, K. Starke, K. Düvel, and G.H. Braus. 2001. Regulation of the Aspergillus nidulans hisB gene by histidine starvation. Curr Genet 38: 314-322.

Casqueiro, J., S. Gutierrez, O. Banuelos, M.J. Hijarrubia, and J.F. Martin. 1999. Gene targeting in Penicillium chrysogenum: disruption of the lys2 gene leads to penicillin overproduction. $J$ Bacteriol 181: 11811188.

Corpet, F. 1988. Multiple sequence alignment with hierarchical clustering. Nucleic Acids Res 16: 10881-10890.

Eckert, S.E., E. Kübler, B. Hoffmann, and G.H. Braus. 2000. The tryptophan synthase-encoding trpB gene of Aspergillus nidulans is regulated by the cross-pathway control system. Mol Gen Genet 263: 867-876.

Felenbok, B., M. Flipphi, and I. Nikolaev. 2001. Ethanol catabolism in Aspergillus nidulans: a model system for studying gene regulation. Prog Nucleic Acid Res Mol Biol 69: 149-204.

Firn, R.D. and C.G. Jones. 2000. The evolution of secondary metabolism - a unifying model. Molecular Microbiology 37: 989-994.

Garrad, R., T.M. Schmidt, and J.K. Bhattacharjee. 1994. Molecular and functional analysis of the LYS1 gene of Candida albicans. Infect Immun 62: 5027-5031.

Gentles, S., C.M. Churcher, B.G. Barrell, M.A. Rajandream, and S.V. Walsh. 1995. SwissProt accession number 09694.

Hinnebusch, A.G. 1986. The general control of amino acid biosynthetic genes in the yeast Saccharomyces cerevisiae. CRC Crit Rev Biochem 21: 277-317.

Hoffmann, B., O. Valerius, M. Andermann, and G.H. Braus. 2001. Transcriptional autoregulation and inhibition of mRNA translation of amino acid regulator gene $c p c A$ of filamentous fungus Aspergillus nidulans. Mol Biol Cell 12: 2846-2857.

Hönlinger, C., W.A. Hampel, M. Rohr, and C.P. Kubicek. 1988. Differential effects of general amino acid control of lysine biosynthesis on penicillin formation in strains of Penicillium chrysogenum. $J$ Antibiot (Tokyo) 41: 255-257.

Hönlinger, C. and C.P. Kubicek. 1989. Regulation of $\delta$-(L- $\alpha$-aminoadipyl)-L-cysteinyl-D-valine and isopenicillin $\mathrm{N}$ biosynthesis in Penicillium chrysogenum by the alpha- aminoadipate pool size. FEMS Microbiol Lett 53: $71-75$.

Jaklitsch, W.M., W. Hampel, M. Rohr, C.P. Kubicek, and G. Gamerith. 1986. $\alpha$-Aminoadipate pool concentration and penicillin biosynthesis in strains of Penicillium chrysogenum. Can J Microbiol 32: 473-480.

Käfer, E. 1977. Meiotic and mitotic recombination in Aspergillus and its chromosomal aberrations. Adv Genet 19: 131-133.

Kennedy, J. and G. Turner. 1996. $\delta$-(L- $\alpha$-aminoadipyl)-L-cysteinyl-D-valine synthetase is a rate limiting enzyme for penicillin production in Aspergillus nidulans. Mol Gen Genet 253: 189-197.

Krappmann, S., K. Helmstaedt, T. Gerstberger, S. Eckert, B. Hoffmann, M. Hoppert, G. Schnappauf, and G.H. Braus. 1999. The aroC gene of Aspergillus nidulans codes for a monofunctional, allosterically regulated chorismate mutase. J Biol Chem 274: 22275-22282. 
Lee, B.S. and J.W. Taylor. 1990. Isolation of DNA from fungal mycelia and single spores. In PCR protocols: $A$ guide to methods and applications (ed. M.A. Innis, D.H. Gelfand, J.S. Sninsky, and T.J. White), pp. 282-287. Academic Press Inc, San Diego.

Lu, Y., R.L. Mach, K. Affenzeller, and C.P. Kubicek. 1992. Regulation of $\alpha$-aminoadipate reductase from Penicillium chrysogenum in relation to the flux from $\alpha$-aminoadipate into penicillin biosynthesis. Can $J$ Microbiol 38: 758-763.

Luengo, J.M. and M.A. Penalva. 1994. Penicillin biosynthesis. In Aspergillus: 50 years on. Prog Ind Microbiol (ed. S.D. Martinelli and J.R. Klinghorn), pp. 603-638. Elsevier, Amsterdam.

MacCabe, A.P., M.B. Riach, S.E. Unkles, and J.R. Kinghorn. 1990. The Aspergillus nidulans npeA locus consists of three contiguous genes required for penicillin biosynthesis. EMBO J 9: 279-287.

Martin, J.F. 1998. New aspects of genes and enzymes for $\beta$-lactam antibiotic biosynthesis. Appl Microbiol Biotechnol 50: 1-15.

Martin, J.F. 2000. Molecular control of expression of penicillin biosynthesis genes in fungi: regulatory proteins interact with a bidirectional promoter region. Journal of Bacteriology 182: 2355-2362.

Mathieu, M. and B. Felenbok. 1994. The Aspergillus nidulans CREA protein mediates glucose repression of the ethanol regulon at various levels through competition with the ALCR- specific transactivator. EMBO J 13: 4022-4027.

Mavrothalassitis, G., G. Beal, and T.S. Papas. 1990. Defining target sequences of DNA-binding proteins by random selection and PCR: determination of the GCN4 binding sequence repertoire. DNA Cell Biol 9: 783-788.

Natarajan, K., M.R. Meyer, B.M. Jackson, D. Slade, C. Roberts, A.G. Hinnebusch, and M.J. Marton. 2001. Transcriptional profiling shows that Gcn $4 p$ is a master regulator of gene expression during amino acid starvation in yeast. Mol Cell Biol 21: 4347-4368.

Ogawa, H., T. Hase, and M. Fujioka. 1980. Amino acid sequence of a peptide containing an essential cysteine residue of yeast saccharopine dehydrogenase (L-lysine-forming). Biochim Biophys Acta 623: 225-228.

Oliphant, A.R., C.J. Brandl, and K. Struhl. 1989. Defining the sequence specificity of DNA-binding proteins by selecting binding sites from random-sequence oligonucleotides: analysis of yeast GCN4 protein. $\mathrm{Mol}$ Cell Biol 9: 2944-2949.

Perez-Esteban, B., M. Orejas, E. Gomez-Pardo, and M.A. Penalva. 1993. Molecular characterization of a fungal secondary metabolism promoter: transcription of the Aspergillus nidulans isopenicillin $\mathrm{N}$ synthetase gene is modulated by upstream negative elements. Mol Microbiol 9: 881-895.

Punt, P.J., M.A. Dingemanse, B.J. Jacobs-Meijsing, P.H. Pouwels, and C.A. van den Hondel. 1988. Isolation and characterization of the glyceraldehyde-3-phosphate dehydrogenase gene of Aspergillus nidulans. Gene 69: 49-57.

Ramon, D., L. Carramolino, C. Patino, F. Sanchez, and M.A. Penalva. 1987. Cloning and characterization of the isopenicillin $\mathrm{N}$ synthetase gene mediating the formation of the B-lactam ring in Aspergillus nidulans. Gene 57: 171-181.

Sachs, M.S. 1996. General and cross-pathway controls of amino acid biosynthesis. In The Mycota III. Biochemistry and Molecular Biology (ed. R. Brambl and G.A. Marzluf), pp. 315-345. Springer, Berlin.

Schenk, W.A. 2000. Isopenicillin-N-Synthase: Ein Enzym bei der Arbeit. Angewandte Chemie 19: 3551-3554.

Steidl, S., P. Papagiannopoulos, O. Litzka, A. Andrianopoulos, M.A. Davis, A.A. Brakhage, and M.J. Hynes. 1999. AnCF, the CCAAT binding complex of Aspergillus nidulans, contains products of the hapB, $h a p C$, and hapE genes and is required for activation by the pathway-specific regulatory gene amdR. Mol Cell Biol 19: 99-106.

Strittmatter, A.W., S. Irniger, and G.H. Braus. 2001. Induction of jlbA mRNA synthesis for a putative bZIP protein of Aspergillus nidulans by amino acid starvation. Curr Genet 39: 327-334.

Then Bergh, K. and A.A. Brakhage. 1998. Regulation of the Aspergillus nidulans penicillin biosynthesis gene acvA $(p c b A B)$ by amino acids: implication for involvement of transcription factor PACC. Appl Environ Microbiol 64: 843-849.

Valerius, O., O. Draht, E. Kübler, K. Adler, B. Hoffmann, and G.H. Braus. 2001. Regulation of hisHF transcription of Aspergillus nidulans by adenine and amino acid limitation. Fungal Genet Biol 32: 21 31.

Waring, R.B., G.S. May, and N.R. Morris. 1989. Characterization of an inducible expression system in Aspergillus nidulans using alcA and tubulin-coding genes. Gene 79: 119-130.

Weidner, G., B. Steffan, and A.A. Brakhage. 1997. The Aspergillus nidulans lys $F$ gene encodes homoaconitase, an enzyme involved in the fungus-specific lysine biosynthesis pathway. Mol Gen Genet 255: 237-247.

Weidner, G., S. Steidl, and A.A. Brakhage. 2001. The Aspergillus nidulans homoaconitase gene lys $F$ is negatively regulated by the multimeric CCAAT-binding complex AnCF and positively regulated by GATA sites. Arch Microbiol 175: 122-132. 
Whiteman, P.A., E.P. Abraham, J.E. Baldwin, M.D. Fleming, C.J. Schofield, J.D. Sutherland, and A.C. Willis. 1990. Acyl coenzyme A: 6-aminopenicillanic acid acyltransferase from Penicillium chrysogenum and Aspergillus nidulans. FEBS Lett 262: 342-344.

Xuan, J.W., P. Fournier, N. Declerck, M. Chasles, and C. Gaillardin. 1990. Overlapping reading frames at the LYS5 locus in the yeast Yarrowia lipolytica. Mol Cell Biol 10: 4795-4806.

Zabriskie, T.M. and M.D. Jackson. 2000. Lysine biosynthesis and metabolism in fungi. Nat Prod Rep 17: 8597. 
Chapter 3 


\section{The COP9 signalosome is an essential regulator of development in Aspergillus nidulans}

\subsection{Abstract}

The COP9 signalosome (CSN) was identified as an essential regulator of development resulting in cleistothecia, the sexual fruit bodies of the filamentous fungus Aspergillus nidulans. The deduced amino acid sequences of two genes, $\operatorname{csn} D$ and $\operatorname{csn} E$, show up to $38 \%$ and $53 \%$ identities to the fourth and fifth CSN subunits of higher eukaryotes. The A. nidulans CSND protein is accumulated in the nucleus and interacts with CSNE in a yeast two-hybrid assay. Deletion of either csn open reading frame resulted in viable strains with identical developmental phenotypes which could be complemented by the according wild-type genes. When grown on an air-medium interface, vegetative cells of $A$. nidulans csn deletion strains were impaired in cell polarity and showed changes in secondary metabolism resulting in the accumulation of a red pigment. The csn deletion strains showed disturbed light-dependence of developmental initiation processes, including constitutive repression of the onset of the asexual reproductive cycle in developmentally synchronised and competent mycelia. The $\Delta c s n$ mutants were capable to initiate the sexual cycle and develop primordia of fruit bodies. However, the further maturation and morphogenesis of primordia to sexual fruit bodies was blocked. This developmental arrest could not be overcome by overexpression of the sexual activator velvet (VEA). We conclude that the COP9 signalosome, which is functionally located at the interface of signal-transduction and ubiquitin-dependent proteolysis, is a key regulator in $A$. nidulans which is essential for light-dependent signalling and subsequent sexual development.

\subsection{Introduction}

Prerequisites for development of multicellular eukaryotes are specific control mechanisms for the cell division cycle, cell specialisation, intercellular communication and numerous modifications of metabolic activities. Co-ordination of such diverse cellular processes requires multiple molecular cross-talk mechanisms. One important regulatory system is targeted protein degradation. The main machinery for controlled proteolysis in eukaryotes is the $26 \mathrm{~S}$ proteasome, which exclusively degrades ubiquitinylated proteins (Tyers and Jorgensen 2000). Ubiquitinylation of the target proteins is mediated in an enzymatic cascade including E3 ubiquitin ligase complexes. The activity of E3 ubiquitin ligases towards its substrate proteins depends on the reversible conjugation of the 
small ubiquitin-like protein Rub1/Nedd8 to their cullin subunit, a process termed neddylation (Ohh et al. 2002), and on the phosphorylation status of the substrate. A proposed modulator of E3 ubiquitin ligase activity is the constitutive photomorphogenesis complex $\underline{9}$ (COP9), termed COP9 signalosome (CSN). Several studies suggest that the CSN directly interacts with E3 ubiquitin ligases (Schwechheimer et al. 2001; Suzuki et al. 2002) and modulates E3 ubiquitinylation activity by at least two distinct enzymatic activities which are probably associated (Bech-Otschir et al. 2002): a deneddylation function contributes to deconjugation of Rub1/Nedd8 from the E3 ligase cullin subunit (Lyapina et al. 2001; Zhou et al. 2001; Yang et al. 2002), and a kinase function can phosphorylate E3 substrate proteins (Seeger et al. 1998; Bech-Otschir et al. 2001).

The COP9 signalosome, the lid of the 26S proteasome (LID) and the eukaryotic translation initiation factor $\underline{3}$ (elF3), are regulatory multiprotein complexes whose components are characterised through the specific $\mathrm{PCl}$ (proteasome, COP9, elF3) or MPN (Mpr1p, Pad1 N-terminal) protein domains (Glickman et al. 1998; Kapelari et al. 2000; Kim et al. 2001). The eight subunits of the COP9 signalosome are highly conserved in higher eukaryotes. In mammalian cells, the CSN is involved in several cellular processes, for example the control of hormone signalling and tumor growth, by regulation of c-Jun and p53 protein levels (Li et al. 2000; Pollmann et al. 2001). In insects and plants, the COP9 signalosome is an essential regulator of development and malfunction of the CSN results in a developmental block that leads to post-embryonic lethality: Arabidopsis thaliana seedlings with defect in the CSN show a constitutive photomorphogenic phenotype and die after several days (Wei et al. 1994), and mutant larvae of Drosophila melangolaster pupate but never undergo morphogenesis (Wei et al. 1994; Freilich et al. 1999). To date, six subunits of the COP9 signalosome have been identified in Schizosaccharomyces pombe, though the role of this complex in fission yeast is not essential and seems restricted to the time control of the cell cycle and sensitivity towards ultraviolet light (Mundt et al. 1999; Mundt et al. 2002). In Saccharomyces cerevisiae the CSN seems not to exist in this form, only a protein with vague similarity to subunit 5 was found (Wei and Deng 1999).

The filamentous ascomycete Aspergillus nidulans is a model organism for the study of regulatory networks that control metabolic pathways, differentiation and development in eukaryotes. In passing through different phases of growth and development, this fungus establishes highly specialised cell types and produces various secondary metabolites. After germination, multinucleate hyphae are formed. They require a minimum time of 16 to $20 \mathrm{~h}$ of vegetative growth to achieve developmental competence, influenced by different physical factors as temperature and cell density. From this time point on, the mycelium is susceptible to environmental signals and subsequent induction of two propagation cycles (Axelrod et al. 1973; Yager et al. 1982). The asexual reproductive unit is the conidiophore, which forms green-pigmented conidiospores about $24 \mathrm{~h}$ after induction (Adams et al. 1998). The reproductive structures of the sexual cycle, the cleistothecia, require about $80 \mathrm{~h}$ post-induction time for maturation and harbour red-pigmented ascospores. Cleistothecia constitute the most complex 
developmental architecture of $A$. nidulans, and since mutant strains defective in their sexual reproduction are still viable, $A$. nidulans is particularly suited for studies on sexual development (Braus et al. 2002). The first morphological evidence for sexual propagation are small hyphal aggregates, termed nests. These include specialised hyphae producing globose Hülle-cells, probably serving as nurse cells. Other specialised hyphae form spherical structures within the nests, the cleistothecial primordia. One step beyond, a first separation of surrounding hyphae embracing an inner hyphal mass becomes evident in the micro-cleistothecia. Several layers of the surrounding hyphae, flat and highly branched, merge to a continuous and hard, cleisthin-glued envelope, the cleistothecial wall. Within this protecting shell, specialised ascogenous hyphae eventually give rise to ascospores. To our knowledge, the precise timing of the single steps that finally lead to production of ascospores it is not known to date. However, specialised ascogenous hyphae fuse. Subsequent crozier formation and synchronised mitosis result in dikaryotic, binucleate ascus mother cells. These develop after karyogamy, meiosis and two rounds of mitosis into asci that enclose eight binuclear ascospores. A mature $A$. nidulans cleistothecium is up to $200 \mu \mathrm{m}$ in diameter and contains approximately 80000 red-pigmented ascospores (Hermann et al. 1983; Kirk and Morris 1991; Champe et al. 1994; Wu and Miller 1997).

Studies on molecular regulation of the reproductive cycles in $A$. nidulans revealed several transcription factors specific for development. Regulation of asexual propagation (Adams et al. 1998) is primarily driven by a cascade of transcriptional activators, with two of them as the main players: BRLA is essential for a developmental switch from apical growth to swelling of the vesicle and ABAA is required for the switch from sterigmata budding to formation of conidiospores. They trigger the expression of downstream structural genes required for asexual development. By contrast, sexual development seems not to be attributed to a single sequential regulatory pathway (Braus et al. 2002). Several acleistothecial A. nidulans strains defective in distinct developmental steps could be isolated. So far, four transcription factors essential for sexual development have been characterised. No visible sexual structures are produced in $n s d D$ (never in sexual development) (Han et al. 2001), or stuA (stunted) mutant strains (Wu and Miller 1997), whereas mutants of ste $A$ (sterile) or medA (medusa) are acleistothecial but do produce the auxiliary Hülle cells (Clutterbuck 1969; Vallim et al. 2000). Notably, stu $A$ and medA mutant strains additionally produce aberrant conidiophores due to altered spatiotemporal expression of $a b a A$ and/or brlA (Miller et al. 1992; Busby et al. 1996). Several A. nidulans strains defective in regulation of amino acid biosynthesis exhibit a reversible block at the level of micro-cleistothecia due to elevated levels of the cross-pathway transcriptional activator CPCA (Eckert et al. 2000; Hoffmann et al. 2000; Busch et al. 2001). Furthermore, several mutants with regard to karyogamy, meiosis and ascosporogenesis can build cleistothecial shells which are empty (Swart et al. 2001).

Knowledge about the regulatory system that drives the initial developmental decisions in $A$. nidulans is scarce. The $v e A$ gene product connects light as external induction signal with development. Light in combination with aeration as another 
external signal direct the differentiation towards the asexual cycle, whereas absence of light and increased partial pressure of carbon dioxide favour sexual propagation (Han et al. 1994; Timberlake and Clutterbuck 1994). Strains carrying the mutant veA1 allele (many commonly used laboratory strains) abolish lightdependence of conidiation and constitutively produce 20fold more conidia and 5 fold less cleistothecia. The time course of sexual development is about doubled in veA1 mutant strains (Mooney and Yager 1990; Champe et al. 1994). Additionally, hormone-like signal molecules like the Psi factors (precocious sexual inducer) (Champe et al. 1987; Champe and el-Zayat 1989; Lewis and Champe 1995), the FluG factor (Yager et al. 1998) and auxin (Eckert et al. 1999) affect the developmental capabilities of the fungus.

With $\operatorname{csn} D$ and csnE, two components of the COP9 signalosome of $A$. nidulans were identified in this study. Deletion of either csn gene resulted in viable strains impaired in induction of development and maturation of primordia to cleistothecia. This first description of the COP9 complex in a filamentous fungus can serve as a basis in studying the regulatory function in development of this conserved multiprotein complex in an easily amenable, eukaryotic microorganism.

\subsection{Experimental Procedures}

\subsubsection{Growth conditions}

A. nidulans strains were cultivated at $37^{\circ} \mathrm{C}$ in or on minimal medium $(50 \mathrm{mM}$ glucose, $70 \mathrm{mM} \mathrm{NaNO}_{3}, 7 \mathrm{mM} \mathrm{KCl}, 11.2 \mathrm{mM} \mathrm{KH}_{2} \mathrm{PO}_{4}(\mathrm{pH} 5.5), 2 \mathrm{mM} \mathrm{MgSO}_{4}$, trace elements) (Bennett and Lasure 1991) supplemented as described (Käfer 1977). Vegetative mycelia were obtained from submerged liquid culture. Development tests were performed on agar plates with either confluent or point-inoculated ascospores or with mycelia that were pre-grown in submerged culture to synchronise the cell material at the stage of developmental competence. Development was allowed by an medium-air interface. We induced asexual sporulation by incubation on non-sealed plates in continuous white light and cleistothecia formation via oxygen-limiting conditions on tape-sealed plates in the dark (Clutterbuck 1974). Strains of Saccharomyces cerevisiae were grown at $30^{\circ} \mathrm{C}$ under non-selective conditions in YPD or in selective YNB media supplemented as described (Guthrie and Fink 1991).

\subsubsection{A. nidulans physiological studies}

Radial growth tests were performed with about 500 conidiospores centred on an agar plate. Colony growth was recorded as colony diameter. For conidiospore quantification (Bussink and Osmani 1998), $4 \mathrm{ml}$ warm minimal medium containing $0.6 \%$ agar and about $1 \times 10^{6}$ spores were poured on solid medium. After $48 \mathrm{~h}$, the top layer was excised with the end of a $1 \mathrm{ml}$ tip (about $1 \mathrm{~cm}^{2}$ ), placed in $0.5 \mathrm{ml}$ saline and vortexed for $30 \mathrm{~min}$ before spores were counted in a hemacytometer. 


\subsubsection{Molecular methods}

E. coli, S. cerevisiae and A. nidulans transformations were performed as described (Inoue et al. 1990; Elble 1992; Eckert et al. 2000). To obtain homogenised cell material, A. nidulans mycelia were filtered through sterile miracloth (Calbiochem, LaJolla, CA USA), frozen in liquid nitrogen and pulverised with a mortar and pestle. RNA was isolated from a volume of $100 \mu$ ground mycelia mixed with $1 \mathrm{ml}$ Trizol $^{\mathrm{TM}}$ (Gibco BRL Life Technologies, Gaithersburg, MD, USA) as recommended by the manufacturer. Genomic DNA was isolated from a volume of $1 \mathrm{ml}$ mycelia (Lee and Taylor 1990). Standard techniques were applied for Northern and Southern hybridisation experiments (Southern 1975; Rave et al. 1979) with ${ }^{32} \mathrm{P}$-labelled probes employing the HexaLabel ${ }^{\mathrm{TM}}$ DNA Labelling Kit (MBI Fermentas). DNA for the csnD and veA probes was amplified by PCR of plasmids pME2343 and pME2352 with standard primers (T3/T7) and specific primers (SB85: 5'-Xhol-TCA TTG AAT TGA ACA TCT TCG-3' and SB86: 5'-HindIII-ATC GCG CCG GAT ACG GAC ACG-3'). DNA for the gpdA probe was cut from plasmid pAN5-22 (EcoRI/BamHI). RNA quantities were standardised according to the $16 \mathrm{~S}$ and $23 \mathrm{~S}$ rRNA signals on the ethidium bromide-stained gel, quantified via the Image Station 440CF (Kodak, Rochester, NY, USA). Signal intensities of autoradiographies were quantified using a bioimager and MacBAS v2.5(E) from Fuji (Tokyo, Japan).

\subsubsection{REMI mutagenesis}

Restriction-enzyme mediated integration (REMI) (Schiestl and Petes 1991; Sanchez et al. 1998) of $A$. nidulans strain R99 was performed with $5 \mu \mathrm{g}$ of vector pME1510. pME1510 was constructed from pAN8-1 (Punt and van den Hondel 1992) with the PCR amplified multiple cloning site of pBluescriptSK+ inserted into the blunt-ended EcoRI restriction site. It confers phleomycin resistance mediated by the ble gene from Streptoalloteichus hindustanus, which is expressed by the gpdA promoter and the $\operatorname{trp} C$ terminator. Selection for the ble gene was achieved by $20 \mathrm{mg}$ phleomycin per I in the medium. About 4200 phleomycin-resistant colonies were generated with 5 to $20 \mathrm{U}$ of the different restriction endonucleases $\mathrm{BamHI}$, Clal, EcoRl, HindllI and Kpnl. Transformation efficiencies reached 150 transformants per $\mu \mathrm{g}$ of vector used. 50 mutants with fluffy, acleistothecial or hypercleistothecial phenotypes were isolated. Strain AGB37 contains a single integration of the vector in the genome. Backcrossing with the cleistothecial wild-type strain R99-6 (pabaA1) showed that the acleistothecial phenotype was linked to the phleomycin resistance. For plasmid rescue, $10 \mu \mathrm{g}$ restriction enzyme-cut genomic DNA was religated and transformed into E. coli SURE cells (Stratagene, LaJolla, CA, USA). Plasmid pME1661 was rescued from AGB37 containing an insert of genomic DNA of $560 \mathrm{bp}$.

\subsubsection{Isolation of genomic and CDNA of $c s n D$ and $c s n E$}

The REMI-rescued fragment of pME1661 was used to identify a $9 \mathrm{~kb}$ Xhol genomic fragment which was subcloned into pBKS. It contains the complete $\operatorname{csn} D$ open 
reading frame in plasmid pME2338. A genomic fragment as probe for csnE was generated by PCR with primer pair SB102 (5'-Xbal-GAG CTA GAG AAT GCT GTT ACC CTG-3') and SB103 (5'-Xbal-CAA AGT CCT CGG CTT TGT TAA GCG-3') deduced from searches in the Monsanto Microbial Sequence Database for Aspergillus nidulans (http://microbial.cereon.com) and cloned into the vector pBSK as plasmid pME2234 via Xbal. Colony hybridisation of a genomic EcoRI sublibrary in pBSK with the pME2234 insert as a probe revealed a $6 \mathrm{~kb}$ genomic EcoRI fragment containing the complete csnE coding region in plasmid pME2237. cDNAs were generated with RT-PCR with a polyT-primer and specific primers (SB57: 5'TAT GAA TCT AAT AGG TAC CAG AGA-3' and SB58: 5'-CAG GAA GAG AAT ATA GTT ACG AGT-3', SB123: 5'-TAT TCT CCA AAT CGA TAA TTA GC-3' and SB124: 5'-CCA TCC AAA TAG ATC TAT ACG G-3') for $c s n D$ and $c s n E$, and ligated via TA-cloning into pBSK resulting in plasmids pME2364 and pME2363, respectively.

\subsubsection{Construction of plasmids for $\boldsymbol{A}$. nidulans manipulation}

Plasmids used and constructed in this study are summarised in Table 4.1. For deletion of $c s n D$, the $3.5 \mathrm{~kb} E c o R \mathrm{l} /$ Hindlll fragment of pME2338 was subcloned into pBluescript II. From the resulting vector pME1762 the flanking region was amplified via PCR with a specific primer pair (SB140: 5'-CGC AGA CGG AAC AAC AGT-3' and SB139: 5'-TTG AAT CAC AGC AGT GCA-3') and blunt end ligated to the $2.6 \mathrm{~kb}$ $E c o R I / S s p l$ pyr-4 expression cassette of vector pRG3 to obtain the deletion cassette vector pME2342. For disruption of $c s n E$, the $6 \mathrm{~kb} B a m H \mathrm{H} / E c o R \mathrm{l}$ fragment of pME2237 was subcloned into pUC19, the 5 ' part of the coding region was removed via Clal / Kpnl and substituted by the $2.6 \mathrm{~kb} E c o R \mathrm{I} / \mathrm{Sspl}$ pyr-4 expression cassette of vector pRG3 via blunt end ligation resulting in vector pME2369. For complementation of $\Delta c s n D$, the $3 \mathrm{~kb} B a m H \mathrm{H} / \mathrm{Sall}$ fragment of pME2338 was subcloned into $\mathrm{pBSK}$, opened with $\mathrm{BamHI} / \mathrm{Xbal}$ and ligated with the $3.3 \mathrm{~kb}$ Bgll/Xbal phleomycin-resistance cassette of pME1510, resulting in plasmid pME2345. Accordingly, for complementation of $\triangle c s n E$, the phleomycin-resistance cassette of pME1510 was subcloned with Xbal and Xhol into pBSK, opened with $X$ bal and blunt end ligated with the pME2237 EcoRI fragment resulting in plasmid pME2423. For overexpression from the A. nidulans alcA promoter, the csnD open reading frame was PCR-amplified from pME2338 with a specific primer pair containing Kpnl and BamHI restriction sites (SB87: 5'-Kpnl-ATG CCA TCC CAA AAG ATA ATC TCC-3' and SB89: 5'-BamHI-TCA ACG TAC CAG ATG GCC C-3') and cloned into pME1565 as plasmid pME2354 (PalcA:csnD:This2B; pyr-4). Additionally, the alcA promoter of pME1565 (PalcA:MCS:This2B; pyr-4) was substituted with the niiA promoter from pME2341 via EcoRl and opened with Kpnl to insert the $c s n D$-PCR fragment with $K p n l$ sites in the specific primer pair SB87 (5'Kpnl-ATG CCA TCC CAA AAG ATA ATC TCC-3') and SB125 (5'-Kpnl-TCA ACG TAC CAG ATG GCC C-3') resulting in plasmid pME2361 (PniiA:CsnD; pyr-4). The complete $c s n D$ open reading frame was PCR-amplified from pME2338 (SB87: 5'Kpnl- ATG CCA TCC CAA AAG ATA ATC TCC-3' and SB88: 5'-Kpnl- ACG TAC CAG ATG GCC CTC G-3') and fused in frame to the GFP into plasmid pMCB32 
(Fernandez-Abalos et al. 1998) by using the inserted Kpnl sites downstream of the alcA promoter resulting in plasmid pME2353.

Tab. 4.1: Plasmids

\begin{tabular}{|c|c|c|}
\hline plasmid & description & reference \\
\hline pAN8-1 & bleo resistance cassette $\left(a m p^{R} ;\right.$ PgpdA:ble:TtrpC) & Punt and v.d. Hondel 1992 \\
\hline pBSK & pBluescript ${ }^{\circledast I}$ : cloning vector (amp & Stratagene \\
\hline pEG202 & 2-hybrid BD (amp $p_{0}^{\mathrm{P}}$ PADH:lexA:TADH, HIS3, $\left.2 \mu \mathrm{m}\right)$ & Golemis and Brent 1996 \\
\hline pJG4-5 & 2-hybrid AD (amp $\left.{ }^{\mathrm{R}} ; \mathrm{PGAL1:B42:TADH}, \mathrm{TRP} 1,2 \mu \mathrm{m}\right)$ & Gyuris et al. 1993 \\
\hline pMCB32 & GFP vector $(a m p$; PalcA:gfp) & Fernandez-Abalos et al. 1998 \\
\hline pME1510 & REMI vector (amp ${ }^{R}$; pBSK-MCS; PgpdA:ble:TtrpC) & this work \\
\hline pME1565 & overexpression (amp $;$ pyr-4; PalcA-MCS-This2) & this work \\
\hline pME1661 & REMI-rescue AGB37 (pME1510:500 bp insert) & this work \\
\hline pME1762 & $\operatorname{csn} D$ genomic (EcoRI/Hindlll fragment of pME2338) & this work \\
\hline pME2234 & csnE probe (609 bp csnE PCR-fragment in pBSK) & this work \\
\hline pME2237 & csnE genomic ( $6 \mathrm{~kb}$ fragment $E c o R I$ in pBSK) & this work \\
\hline pME2338 & $\operatorname{csn} D$ genomic (10 kb fragment $X$ hol in pBKS) & this work \\
\hline pME2341 & niiA/niaD promoter $\left(a m p^{R} ;\right.$ Pniia-PniaD) & this work \\
\hline pME2342 & $\operatorname{csnD}$ deletion $\left(a m p^{R} ; \mathrm{P}_{c s n D}: p y r-4: \mathrm{T}_{c s n D}\right.$ in pME1762) & this work \\
\hline pME2343 & csnD probe (Clal/Notl fragment of pME2338) & this work \\
\hline pME2345 & $\operatorname{csn} D$ complementation (PgpdA:ble:TtrpC; $c s n D)$ & this work \\
\hline pME2352 & $v e A$ probe (500 bp veA PCR fragment in pBSK) & this work \\
\hline pME2353 & GFP fusion for $\operatorname{csn} D$ (PalcA::csnD::GFP) & this work \\
\hline pME2354 & $\operatorname{csn} D$-overexpression (amp ${ }^{\mathrm{R}} ;$ pyr-4; PalcA-csnD-This2) & this work \\
\hline pME2355 & $c s n D$-bait $\left(a m p^{\mathrm{R}} ;\right.$ PADH:lexA:TADH, HIS3, $\left.2 \mu \mathrm{m}\right)$ & this work \\
\hline pME2357 & 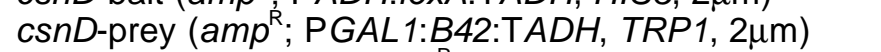 & this work \\
\hline pME2361 & $c s n D$-overexpression $\left(a m p^{\mathrm{R}} ;\right.$ pyr-4; PniiA-csnD-This2) & this work \\
\hline pME2363 & cSnE cDNA (in pBSK) & this work \\
\hline pME2364 & $c s n D$ cDNA (in pBSK) & this work \\
\hline pME2369 & csnE partial deletion (pyr-4 in pSB50) & this work \\
\hline pME2370 & csnE-bait (csnE-cDNA EcoRI in pEG202) & this work \\
\hline pME2371 & csnE-prey (csnE-cDNA EcoRI in pJG4-5 & this work \\
\hline pME2423 & csnE complementation (PgpdA:ble:TtrpC; $c s n E)$ & this work \\
\hline pRG3 & pyr-4 marker $\left(a m p^{\mathrm{R}} ;\right.$ pyr-4) & Waring et al. 1 \\
\hline pUC19 & cloning vector $\left(a m p^{R} ;\right.$ MCS $)$ & Vieira and Messing 1982 \\
\hline
\end{tabular}

\subsubsection{A. nidulans strain construction}

Strains used and constructed in this study are summarised in Table 4.2. As wildtype strains, $A$. nidulans AGB152 and AGB10 were used for a veA and veA1 genetic background, respectively. Both strains were transformed with vector $p R G 3$ resulting in control strains AGB160 and AGB162. Deletion of $c s n D$ was achieved with the deletion cassette of the Xbal-linearised plasmid pME2342 in A. nidulans strains AGB152 and AGB10, resulting in $\triangle c s n D$ strains AGB195 and AGB192, respectively. For deletion of $c s n E$, the Xbal-linearised vector pME2369 was transformed into AGB152 resulting in strain AGB209. For complementation of the csn deletions, pME2345 was transformed into AGB195, AGB192 and AGB209, resulting in strains AGB203, AGB193 and AGB211, respectively. The $c s n D$ deletion was combined with the veA overexpression by transformation of plasmid pME2342 into strain AGB221, the latter one was constructed by a series of crossing experiments between OVAR5, DVAR1, A4 and GR5 (Tab. 4.2.). The csnD overexpression vectors pME2354 and pME2361 were ectopically integrated into $A$. nidulans wild-type strains $A G B 152$ and AGB10 via the pyr-4 marker resulting in strains AGB205 and AGB206, respectively. For localisation studies, ectopic integration of the GFP- 
plasmid pME2353 in A. nidulans strain AGB152 resulted in strain AGB197. All mutant strains were back-crossed to the genetic wild-type strain for purification. Homologous integration of the marker expression cassettes and ectopic integration of plasmids were verified by diagnostic PCR and Southern hybridisation analyses.

Tab. 4.2: A. nidulans strains

\begin{tabular}{|c|c|c|}
\hline strain & genotype & reference: construction \\
\hline A4 & glasgow wild-type & FGSC $^{*}$ \\
\hline AGB10 & pyrG98, pyroA4; veA1 & (Eckert et al. 2000) \\
\hline AGB37 & nsd::bleo & this work: R99 + pME1510 \\
\hline AGB152 & pyroA4, pyrG98 & this work: $A 4 \times$ GR5 \\
\hline AGB160 & pyroA4, pyrG98/pyr-4+ & this work: AGB152 + pRG3 \\
\hline AGB162 & pyroA4, pyrG98/pyr-4 $4^{+}$; veA1 & this work: AGB10 + pRG3 \\
\hline AGB192 & pyroA4; pyrG98/pyr-4 ${ }^{+} \Delta c s n D ;$ veA1 & this work: AGB10 + pME2342 \\
\hline AGB193 & pyroA4; pyrG98/pyr-4 ${ }^{+} \Delta c s n D / c s n D:: b l e o ; ~ v e A 1$ & this work: AGB192 + pME2345 \\
\hline AGB195 & pyroA4, pyrG98/pyr-4 $4^{+} \Delta c S n D$ & this work: AGB152 + pME2342 \\
\hline AGB197 & pyroA4; pyrG98/pyr-4+ PalcA::csnD::gfp & this work: AGB152 + pME2353 \\
\hline AGB203 & pyroA4; pyrG98/pyr- $4^{+} \Delta c s n D / c s n D::$ bleo & this work: AGB195 + pME2345 \\
\hline AGB205 & pyroA4; pyrG98/pyr-4+ PniiA::csnD & this work: AGB152 + pME2361 \\
\hline AGB206 & pyroA4; pyrG98/pyr-4 $4^{+}$PniiA::csnD; veA1 & this work: AGB10 + pME2361 \\
\hline AGB209 & pyroA4; pyrG98/pyr-4 $4^{+} \Delta C S n E$ & this work: AGB152 + pME2369 \\
\hline AGB211 & pyroA4; pyrG98/pyr-4 $4^{+} \Delta c s n E / c s n E::$ bleo & this work: AGB209 + pME2423 \\
\hline AGB220 & $\begin{array}{l}\text { pyrG98/pyr- } 4^{+} \Delta c s n D ; \Delta \arg B: \text { trpC } \Delta \mathrm{B} / \arg B^{+} \\
\text {PniiA::veA; trpC } 801\end{array}$ & this work: AGB222 + pME2342 \\
\hline AGB221 & pyrG98; $\triangle \arg B:: \operatorname{trp} C \Delta \mathrm{B} ; \operatorname{trp} C 801$ & this work: AGB152 x DVAR1 \\
\hline AGB222 & pyrG98; $\Delta$ argB::trpC $\Delta \mathrm{B} / \arg ^{+}$PniiA::veA; trpC801 & this work: AGB221 x OVAR5 \\
\hline DVAR1 & $y A 2$, pabaA $1 ; \Delta \arg B:: \operatorname{trp} C \Delta \mathrm{B} / \arg B^{+} \Delta v e A ; \operatorname{trp} C 801$ & R. Fischer* \\
\hline GR5 & pyrG98, pyroA4; veA1; wA3 & G. May* \\
\hline OVAR5 & 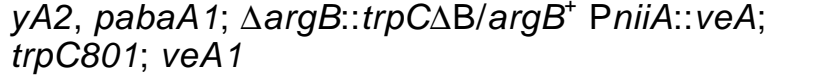 & R. Fischer* \\
\hline R99 & wild-type "arizona" & D. Geiser ${ }^{*}$ \\
\hline R99-6 & pabaA1 & D. Geiser* \\
\hline
\end{tabular}

* Fungal Genetics Stock Center (University of Kansas Medical Center, Kansas City, KS, USA); Dave Geiser (Penn State University, University Park, PA, USA); Gregory May (University of Texas, Houston, TX, USA); Reinhard Fischer (Phillips-Universität Marburg, Germany).

\subsubsection{Microscopy}

Standard morphologic analyses were performed with a Stemi 2000-6 ZEISS binocular and an Axiolab ZEISS light microscope, using a KAPPA digital camera (DX30 ProgressiveScan) and the KAPPA ImageBase software for photography and calibration of magnifications (KAPPA opto-electronics). For GFP localisation, $A$. nidulans was grown over night in liquid $P a l c A$-inducing medium and hyphae were microscoped by either differential interference contrast (DIC) or fluorescence microscopy using a GFP filter set (AHF Analysetechnik AG) under a Axiovert S100 ZEISS microscope. Standard DAPI filter sets were used for visualisation of nuclei stained with 4',6-Diamino-2-phenylindole (DAPI). Photographs were taken using a Xillix Microimager digital camera and the Improvision Openlab software (Improvision). 


\subsubsection{Two-hybrid analysis}

The transcriptional activation of a reporter system with plasmids pEG202 and pJG4-5 was used to identify interacting proteins (Gyuris et al. 1993; Golemis and Brent 1996). The complete $c s n D$ open reading frame was PCR-amplified from pME2338 with specific primers containing flanking Xhol site (SB96: 5'-Xhol- ATG CCA TCC CAA AAG ATA ATC TCC-3' and SB97: 5'-Xhol- TCA ACG TAC CAG ATG GCC CTC-3'). Accordingly, the CSnE cDNA sequence of plasmid pME2363 was amplified with primers flanked by an EcoRI site (SB135: 5'-EcoRI- ATG CAA GCT GCT CAA CTA TCC-3' and SB136: 5'-ECoRI-CTA AGT AGA CTC TAC CGT CTG TIT TC-3'). All PCR fragments were cloned into pEG202 and pJG4-5, resulting plasmids pME2355, pME2370, as 'baits' and pME2357, pME2371 as 'preys'. The constructs were transformed into the yeast two-hybrid reporter strain EGY48-p1840 (MATo, his3, trp1, ura3-52, leu2::pLEU2-LexAop6, URA3::lacZ-LexAop2) (Golemis and Brent 1996). For interaction tests, $1.5 \mathrm{ml}$ of overnight yeast cultures were washed twice with saline, resuspended in $1 \mathrm{ml}$ saline and $10 \mu \mathrm{l}$ of an appropriate dilution was dropped on SC-plates containing $2 \%$ galactose as carbon source for the growth test. For $\beta$-galactosidase tests, the medium was supplemented with leucine $(0.2 \mathrm{~g} / \mathrm{l})$ and covered with a filter paper. Cells were grown for $48 \mathrm{~h}$ at $30^{\circ} \mathrm{C}$. The filter was lifted, shock frozen in liquid nitrogen and applied to a filter soaked with Z-buffer $\left(60 \mathrm{mM} \mathrm{Na} \mathrm{HPO}_{4}, 40 \mathrm{mM} \mathrm{NaH} \mathrm{PO}_{4}, 10 \mathrm{mM} \mathrm{KCl}, 1 \mathrm{mM} \mathrm{MgSO} 4\right)$ supplemented with $40 \mu \mathrm{l} \mathrm{XGal} \mathrm{(1 \%} \mathrm{in} \mathrm{DMF).} \mathrm{The} \mathrm{filters} \mathrm{were} \mathrm{incubated} \mathrm{at} 30^{\circ} \mathrm{C}$ and the reaction was stopped with $\mathrm{Na}_{2} \mathrm{CO}_{3}(1 \mathrm{M})$ after 1 to $24 \mathrm{~h}$.

\subsubsection{Sequence analyses}

For DNA sequence analysis with custom oligonucleotides (Gibco BRL) the Big Dye ${ }^{\mathrm{TM}}$ Terminator kit of the Cycle Sequencing Reaction Mix from Perkin Elmer was used. Sequence analysis was performed on a Perkin Elmer Sequenator 310 with the ABI EditView 1.0.1 software. Further DNA analysis was performed with the Lasergene software from DNASTAR. Saccharopine dehydrogenase peptide sequences were identified using BLAST search of a non-redundant protein database (Altschul et al. 1990). Pairwise alignments and the multiple sequence alignment with hierarchial clustering was performed using the software "CLUSTALW" (Corpet 1988). Identification of protein motifs was performed in silico with PROSITE SCAN at http://hits.isb-sib.ch/cgi-bin/PFSCAN_parser. The nucleotide sequence of the genomic inserts of plasmids pME2338 and pME2237 containing the complete $c s n D$ and $c s n E$ coding regions have been deposited in the GenBank database under GenBank Accession Numbers AF236662 and AY126455, respectively. 


\subsection{Results}

\subsubsection{The $A$. nidulans $c s n D$ gene encodes a $\mathrm{PCl}$ domain protein similar to the fourth subunit of the COP9 signalosome}

We have mutagenised the A. nidulans wild-type strain R99 by the restrictionenzyme mediated integration technique (REMI) to screen for phenotypic defects in the formation of cleistothecia, the sexual fruit bodies of this fungus. REMI is a tagging mutagenesis method aiming to disrupt genes by inserting a marker, which was in this case the ble gene that mediates resistance to phleomycin, into genomic restriction sites (Sanchez et al. 1998). Genetic analysis of one of the mutant strains, AGB37, showed linkage between the single vector integration event conferring phleomycin resistance and the acleistothecial phenotype as proven by Southern and backcrossing experiments. The corresponding plasmid rescued from this strain contained a $560 \mathrm{bp}$ genomic insert. Using this insert as a probe in colony hybridisation experiments, we isolated a genomic $9 \mathrm{~kb}$ Xhol fragment containing an open reading frame of $1227 \mathrm{bp}$ (Fig. 4.1A). Southern hybridisation

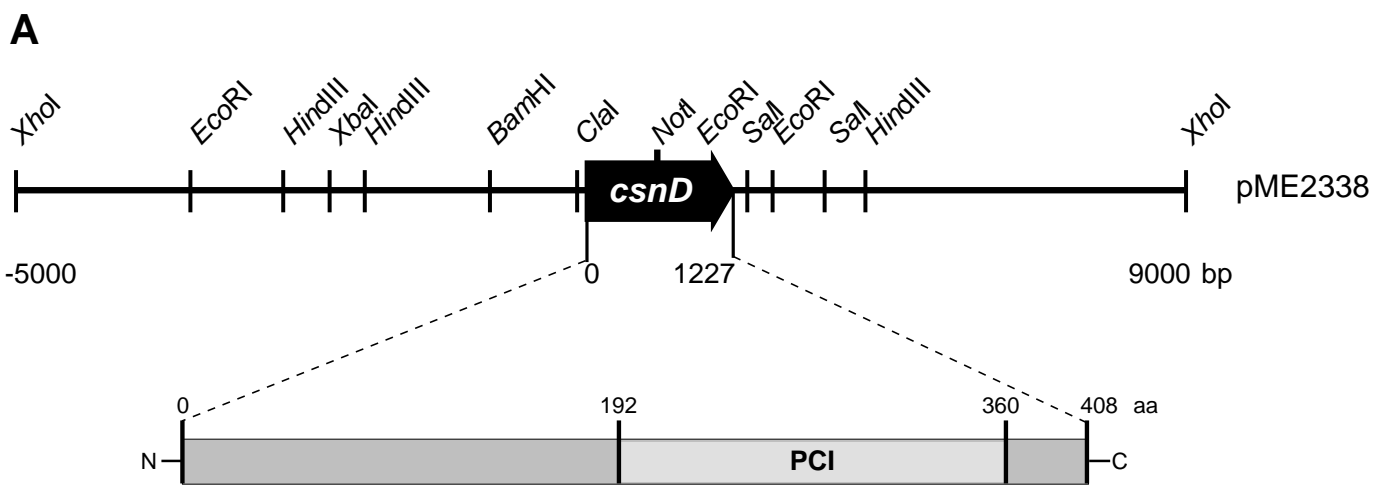

B

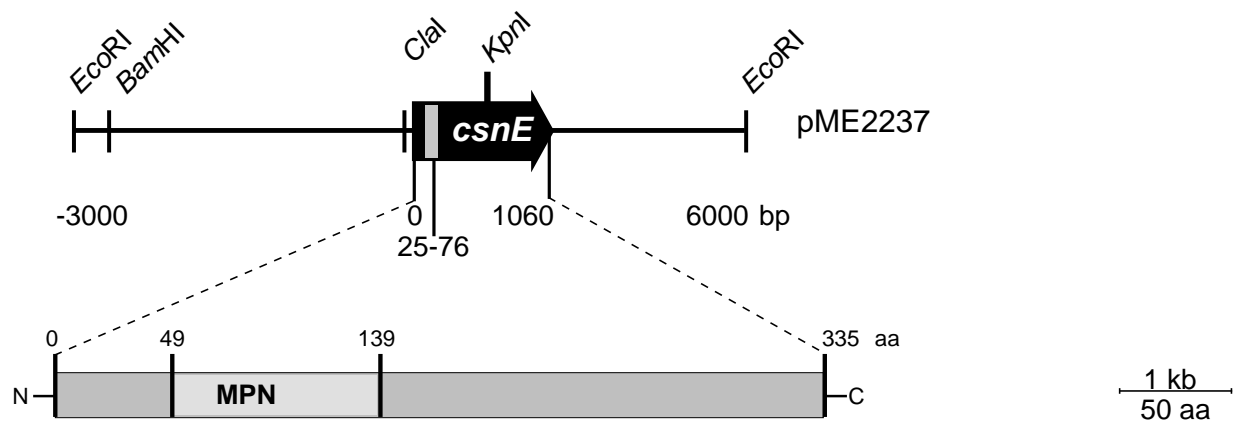

Fig. 4.1: The A. nidulans genes $c s n D$ and $c s n E$ encode $\mathrm{PCl}$ and MPN proteins typical for subunits of the COP9 signalosome. Schemes of the genomic loci of CsnD (GenBank accession number AF236662) (A) and csnE (GenBank accession number AY126455) (B) are shown. The intron of $c S n E$ is indicated by a grey box. Predicted PCl (proteasome, COP9, elF3) and MPN (Mpr1p, Pad1 N-terminal) motifs were identified from the deduced amino acid (aa) sequence by Prosite Scan. 
A

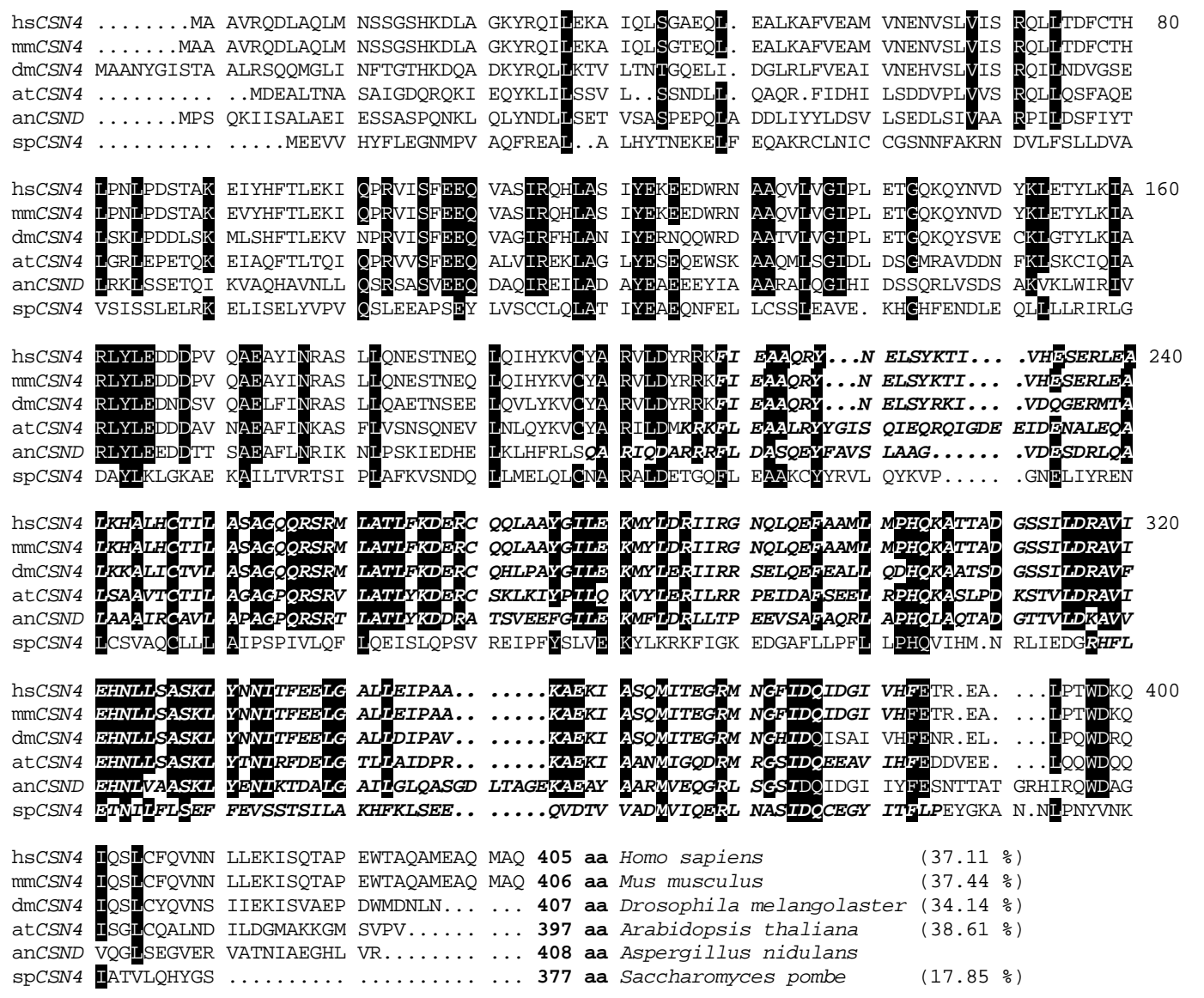

B

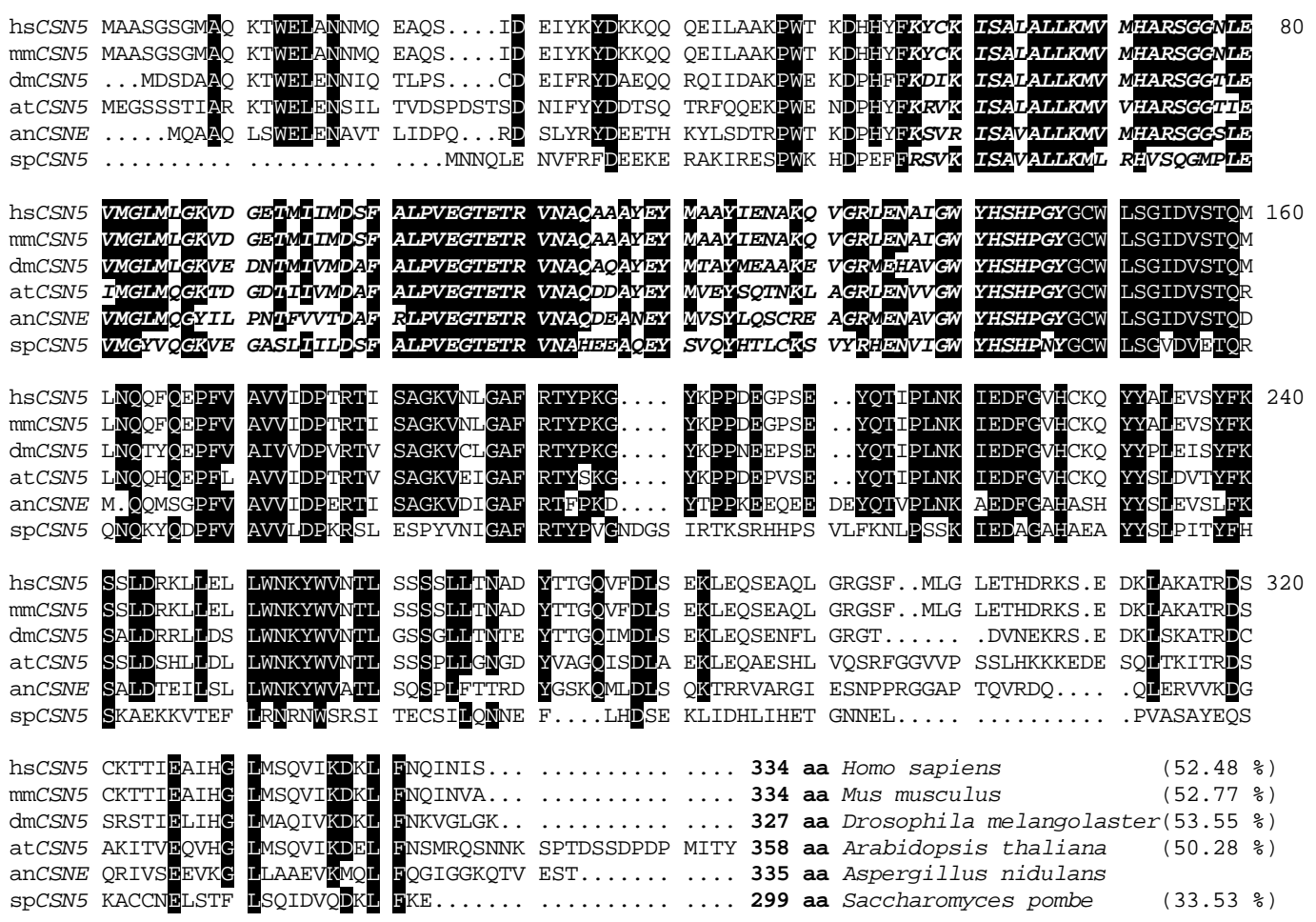

Fig. 4.2: CSND and CSNE show high amino acid identities to corresponding CSN subunits of higher eukaryotes. The multialignments of deduced amino acid sequences of $\operatorname{csn} D(\mathbf{A})$ and $\operatorname{csn} E$ (B) to the corresponding sequences of Homo sapiens, Mus musculus, Drosophila melangolaster, Arabidopsis thaliana and Schizosaccharomyces pombe were performed in silico using ClustalW. Residues identical in five of the sequences are highlighted. The predicted $\mathrm{PCl}$ and MPN domains as identified by Prosite Scan are indicated for each sequence by bold italic print. Percentage of single amino acid identities to the $A$. nidulans sequence is given in parentheses. 
experiments suggest that the respective locus is present in a single copy in the A. nidulans genome (data not shown). A corresponding cDNA was isolated via RTPCR and comparison of both genomic and cDNA nucleotide sequences revealed that the coding region is not interrupted by an intron. From the deduced peptide sequence of 408 amino acids a molecular mass of $44.9 \mathrm{kDa}$ was calculated. The putative protein contained a predicted PCl domain (proteasome, COP9, elF) and displayed identities of up to $38 \%$ to proteins described as the fourth subunit of the COP9 signalosome (CSN) from various organisms (Fig. 4.2A). According to the revised signalosome subunit nomenclature (Deng et al. 2000) the gene was named csnD (GenBank accession number AF236662). Identities were higher when CSND is compared to mammalia, vertebrates and plants than to the ascomycete $S$. pombe. The complete genome sequence of $S$. cerevisiae includes no orthologs of this subunit.

\subsubsection{The $\operatorname{csn} D$ gene is constitutively transcribed through the entire life cycle of $A$. nidulans and its gene product is enriched in the nucleus}

The COP9 signalosome is described as a regulator of developmental transitions in higher eukaryotes (Wei et al. 1994; Freilich et al. 1999). We thus analysed the presence of $\operatorname{csn} D$ transcript in $A$. nidulans in vegetative and differentiated cell material. Strain AGB162, which is wild-type for $\operatorname{csnD}$, was grown in liquid minimal until developmental competence, transferred to solid minimal medium and induced either asexually on unsealed plates or sexually on tape-sealed plates. At harvest, the asexual cultures showed immature and mature conidiophores, respectively. Sexual cultures additionally differentiated Hülle cells, nests and mature cleistothecia. Northern experiments with RNA size standard revealed distinct $\operatorname{csn} D$ transcript signals of about $1.4 \mathrm{~kb}$ in length (Fig. 4.3). Equal amounts of RNA were loaded for each developmental state and $\operatorname{csn} D$ transcript levels were compared to the amount of rRNAs. Specific $\operatorname{csn} D$ mRNA overall signal intensities were comparably low but the ratios relative to the rRNA signals persist in the developing mycelia during all stages. As control, transcriptional levels of the metabolic house-keeping gene gpdA, encoding glyceraldehyde-3-phosphate dehydrogenase (Punt et al. 1990), were monitored which significantly decreased as soon as development was induced.

Further attention was drawn to the localisation of the CSND protein within the hyphal cell compartments by use of the gfp-encoded green fluorescent protein (Fernandez-Abalos et al. 1998). The complete $c s n D$ open reading frame driven by the strong inducible alcA promoter was fused in-frame to the gfp gene. This construct was ectopically integrated into the $A$. nidulans wild-type strain AGB152. Upon either ethanol or cyclopentanone induction of the alcA promoter on solid medium, the resulting strain AGB197 grew and differentiated both asexually and sexually like the wild-type (not shown). When mycelia were grown in submerged liquid culture under inductive conditions, bright signals were detected under the fluorescence microscope, which were absent in the wild-type strain (Fig. 4.4). Thus, this fusion protein consisting of the CSND subunit of the COP9 signalosome and 


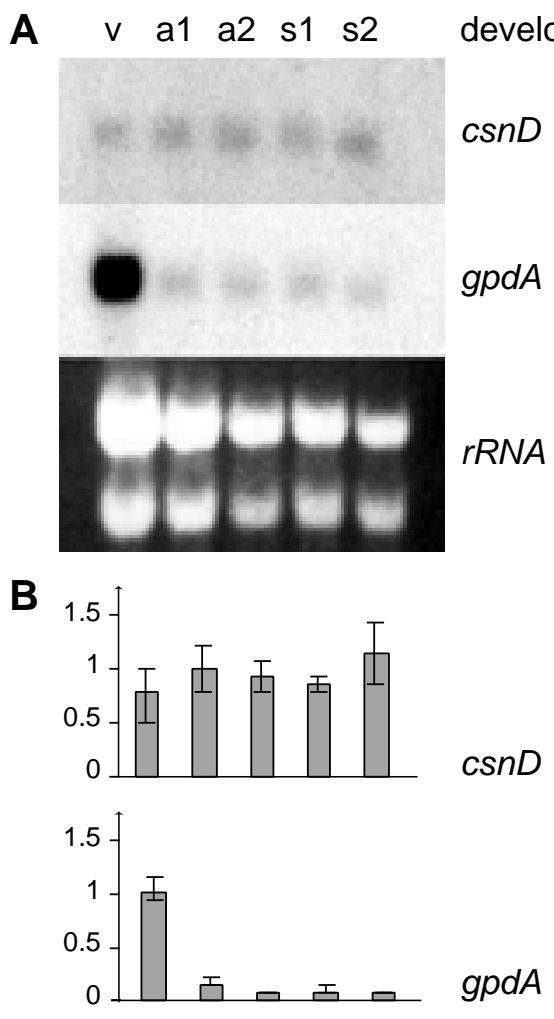

Fig. 4.3: Transcripts of $c s n D$ are expressed in vegetative and differentiated mycelia of A. nidulans. Mycelia of the $A$. nidulans wildtype strain AGB160 were developmentally synchronised by $18 \mathrm{~h}$ vegetative growth (v) in liquid culture. Mycelia were transferred to solid medium for harvest of synchronised asexual (a1 for immature conidiophores; a2 for mature conidia) and sexual ( $\mathrm{s} 1$ for nests including $\mathrm{H}_{s}$ lle cells; s2 for mature cleistothecia) tissue types. RNA was isolated and applied to Northern hybridisation analysis. Specific mRNA signals for $\operatorname{csn} D$ and $g p d A(A)$ were quantified relative to rRNA signal intensities and the values determined from vegetative mycelia were set as one (B).

the green fluorescent protein was expressed in A. nidulans. In the majority of the hyphae, the fluorescence was ubiquitous but accumulated as distinct spots. Nuclei were stained with DAPI and in silico merge of the resulting images revealed that the spots obtained from nuclear stain and GFP matched. Such enrichment of CSN subunits in the nucleus, accompanied by a cytoplasmatic subcomplex of the CSN, was also observed in higher eukaryotes (Tomoda et al. 2002). This analogy in localisation additionally accounts for the identity of CSND as a component of a COP9 signalosome in A. nidulans.

\subsubsection{Deletion of the csnD gene blocks sexual development and causes altered pigmentation and cell morphologies in $A$. nidulans}

A transgenic $A$. nidulans strain carrying a targeted deletion of $\operatorname{csn} D$ was constructed to verify the acleistothecial phenotype of the REMI mutant strain AGB37. The complete open reading frame of $c s n D$ was replaced by a pyr-4 expression cassette in the $A$. nidulans wild-type strain AGB152. The resulting transformants were viable. Homologous integration of the csnD flanking regions into the genome was verified by Southern analysis. After backcrossing of the transformants to the wild-type strain, the resulting deletion strain AGB195 produced no specific $\operatorname{csn} D$ transcripts as proven in Northern hybridisation experiments. Radial colony expansion from a point spore inoculum on an agar surface resulted in similar growth rates with an average of about $0.37 \mathrm{~mm}$ radial vegetative colony growth per hour for the $\operatorname{csn} D$ deletion strain compared to $0.41 \mathrm{~mm} / \mathrm{h}$ for the wild-type at $37^{\circ} \mathrm{C}$. In submerged liquid culture, where $A$. nidulans generally does not induce any development for lack of an air-medium interface (Axelrod et al. 1973), the $\operatorname{csn} D$ deletion strain was viable and did not show obvious anomalies in the morphology of vegetative hyphae. 
However, when the $\operatorname{csn} D$ deletion strain was grown at an air-medium interface for a minimum of 48 hours, two distinct peculiarities of hyphae became obvious: cellular abnormalities and red colouring (Fig. 4.5). When the surface material of A. nidulans colonies was removed by washing the agar plates under the tab, an aberrant reddish compact hyphal mat became visible that partly penetrated the agar surface. To facilitate microscopic analysis, cultures were grown on the surface of liquid medium which revealed the same hyphal traits. The $\operatorname{csn} D$ deletion strain produces, beneath apparently normal filaments, highly branched hyphae consisting of very short cells, indicating a polarity defect. Single hyphae showed a brownish-red pigmentation that was completely absent in mycelia from the wild-type strain which indicates a problem in secondary metabolism. The red colouring appeared in a majority of the aberrant cells, but to a minor extent also in some morphologically wild-type like hyphae and conidiophore stalks. More careful observations of this phenomenon revealed that the centre of a $\operatorname{csn} D$ mutant colony discoloured earliest $48 \mathrm{~h}$ after inoculation. This colouring pattern spread towards the edge of the colony within time. We could not detect major secretion of a dye into the agar medium.

Conidiophore morphology of the $\operatorname{csn} D$ deletion strain resembled that of the wild-type. Single colonies or a point inoculum of this mutant differentiated conidiospores predominantly, but not exclusively, in the centre of the colony. At confluent inoculation, but not in pregrown developmentally competent mycelia (as described below), the number of conidiospores produced after two days was about the same range in both mutant and wild-type strain. Thus, the process of conidiation seemed not generally affected in the csnD deletion mutant AGB195. In contrast to that, the $A$. nidulans $\operatorname{csn} D$ deletion strain showed a severe defect in the sexual propagation cycle (Fig. 4.5). The first morphologically visible stages of the sexual cycle, including formation of Hülle cells and nests, were not affected. Within the nests cleistothecial primordia developed, which morphologically resembled that of the wild-type. The next developmental step, formation of micro-cleistothecia, was never observed in the $\operatorname{csn} D$ deletion strain. Consequently, the mutant strain failed to produce mature cleistothecia with a hard shell and red pigmented ascospores as seen in the wild-type. This specific block in sexual development, together with the polarity and colouring defects, were complemented in strain AGB203 where a genomic wild-type $\operatorname{csn} D$ fragment was ectopically integrated in the $\operatorname{csn} D$ deletion mutant (Fig. 4.6). Thus, all mutant phenotypes were dependent on the $\operatorname{csn} D$ gene. The connection between the red colouring, the morphologically different hyphae and the block in sexual development in the $\operatorname{csn} D$ deletion strain remains to be elucidated.

\subsubsection{An A. nidulans strain lacking the fifth CSN subunit (CSNE) shows a $\triangle c s n D$-like phenotype}

A second component of the proposed multiprotein complex was isolated and deleted to verify the existence of the COP9 signalosome in $A$. nidulans. A nucleotide stretch with similarity to CSN subunit 5 from $A$. thaliana was identified in the A. nidulans genome of the Cereon database. The corresponding genomic DNA 

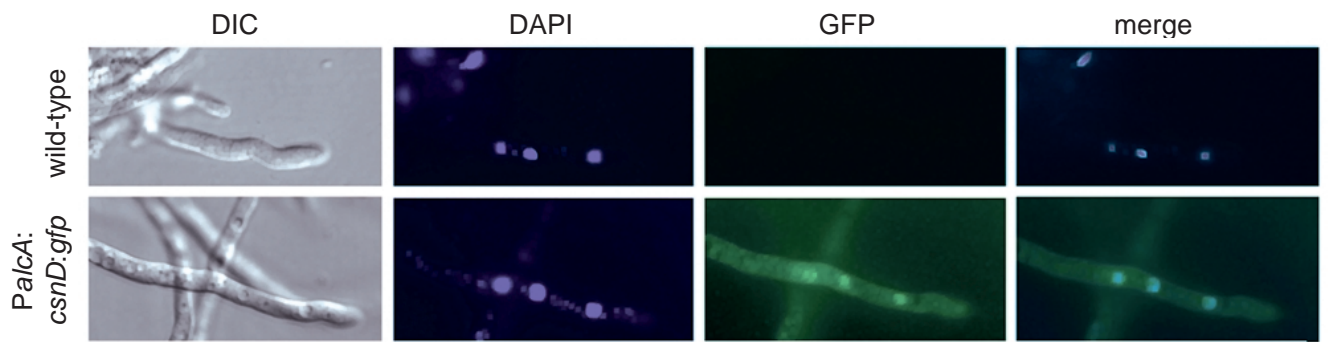

Fig. 4.4: CSND is enriched in the nuclei of $\boldsymbol{A}$. nidulans. $A$. nidulans wild-type strain $A G B 160$ and a strain carrying a PalcA-driven overproduction of a CSND::GFP fusion protein (AGB197) were grown over night in liquid submerged culture under inductive conditions. The CSND:GFP fusion accumulated in the nucleus. Nuclei of liquid mycelia were stained by DAPI and pictures of microscopy were taken with DIC, DAPI fluorescence and GFP fluorescence.
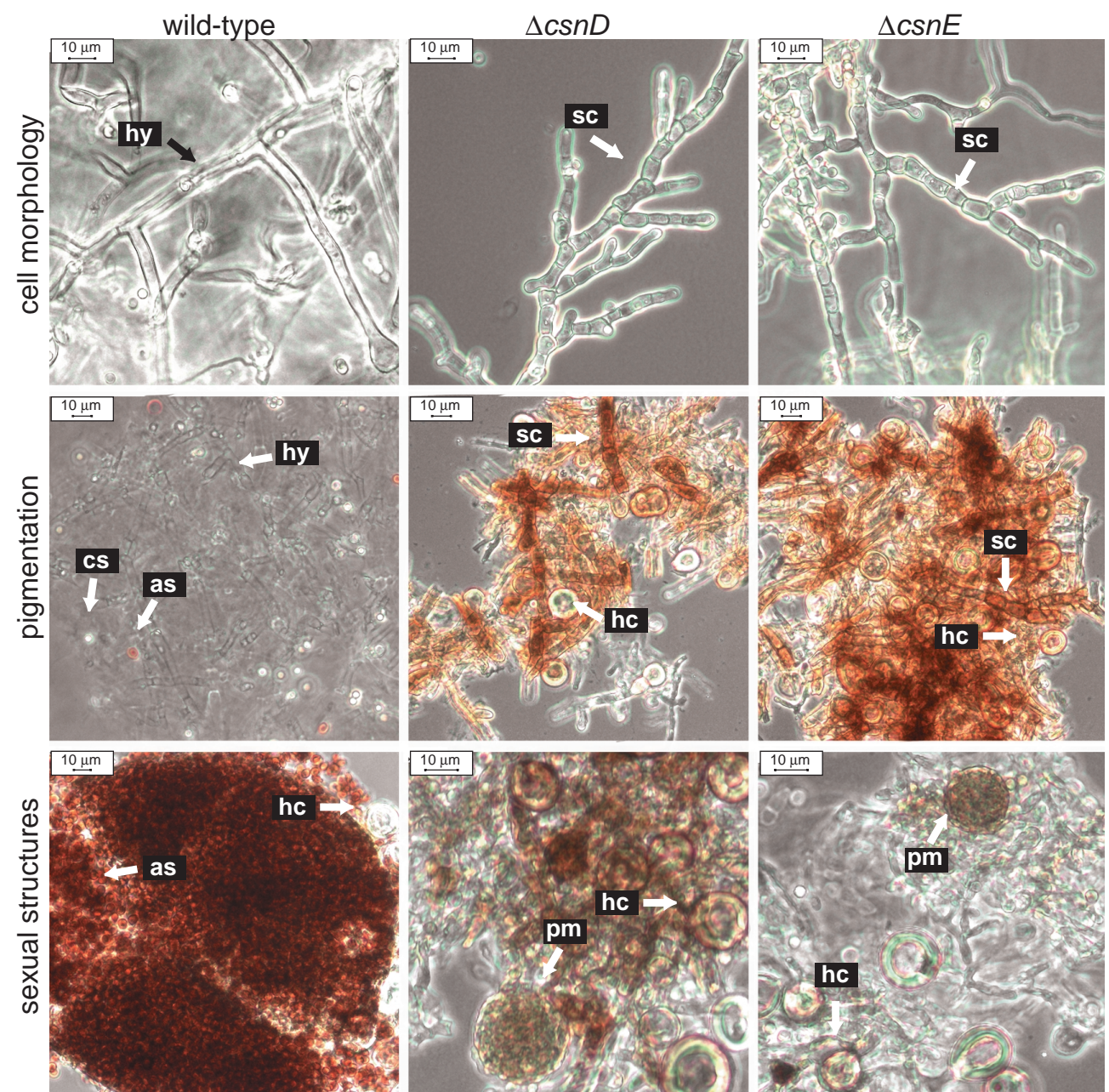

Fig. 4.5: $A$. nidulans strains that lack CSN subunits exhibit pleiotrophic mutant phenotypes. Deletion of $\operatorname{csnD}$ (strain AGB195) or $\operatorname{csnE}$ (strain AGB209) result in identical mutant phenotypes as depicted by microscopic analysis. As control, the wild-type strain AGB160 is shown. (Row 1) Wild-type and mutant strains were grown on a surface of liquid medium to obtain isolated hyphal structures, since hyphae scratched from an agar surface tend to tightly aggregate. The cell morphologies in the csn mutant strains include wild-type vegetative hyphae (hy) and hyperbranched filaments consisting of aberrant short cells (sc), in some cases including a red pigment as seen in the next row. (Row 2) The surface material of the A. nidulans colonies was carefully removed under the water-tap and the hyphal material was the scratched from surface agar layer. Single hyphae in the csn mutant strains, including normal filaments and short cells, synthesise an intensive red coloured pigment. (Row 3) Sexual structures of the strains were collected from the colony surface material. Sexual development of the wild-type strain results in mature cleistothecia with ascospores (as) whereas the csn mutant strains develop only Huelle cells (hc) and primordia (pr). 


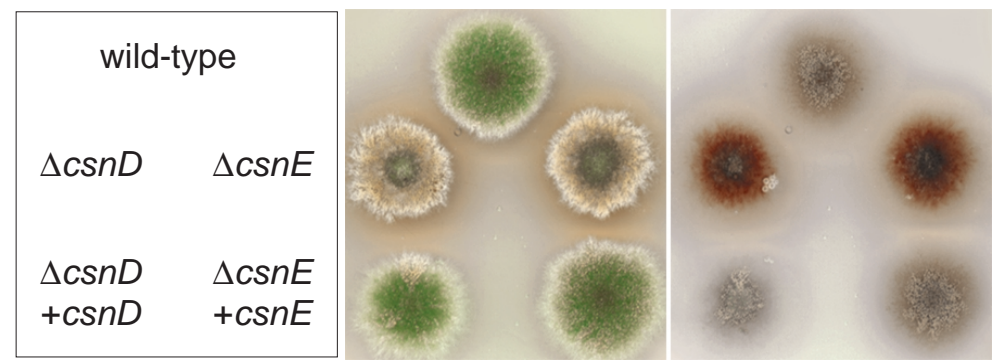

Fig. 4.6: The $A$. nidulans $c s n D$ and csnE deletion strains can be complemented by ectopic integration of the corresponding genomic loci. The $A$. nidulans $\operatorname{csn} D$ and $\operatorname{csn} E$ deletion strains and AGB195 and AGB209, respectively, were complemented with the corresponding wild-type csnD and csnE genes in strains AGB203 and AGB211, respectively. The wild-type strain AGB160 is shown as control. The strains were point inoculated on solid medium and incubated at conditions that allow both asexual and sexual development (left). The csn deletion strains failed to develop mature cleistothecia and produce a compact hyphal mat beneath the surface structures containing aberrant pigmentation. The surface cell material was removed by carefully washing the plates with a cotton ball under the water-tap (right) which uncovered the red pigmentation.

was amplified by PCR using a specific primer pair deduced from this sequence information. This way, we isolated and sequenced a $609 \mathrm{bp}$ fragment with deduced amino acid similarities to CSN subunit 5. This fragment was used as probe in colony hybridisation experiments which revealed a $6 \mathrm{~kb}$ genomic EcoRI fragment containing a coding region of $1060 \mathrm{bp}$ (Fig. 4.1B). Southern hybridisation experiments indicate that the coding region is present with one single copy in the genome (data not shown). The corresponding cDNA was isolated via RT-PCR. Comparison of genomic and cDNA sequences revealed one intron of $51 \mathrm{bp}$. From the deduced peptide sequence of 335 amino acids a molecular mass of $37.8 \mathrm{kDa}$ was calculated. The putative protein showed up to $53 \%$ identity to the fifth subunits of the COP9 complex from various organisms (Fig. 4.2B). Subunit five is the only component of the COP9 signalosome which has, with Rri1p, a putative counterpart in the yeast genome. Rri1p of $S$. cerevisiae exhibits only $20,2 \%$ identities to the A. nidulans CSNE (not shown). Analysis of the amino acid sequence revealed a conserved MPN domain characteristic for subunit 5 of the signalosome. The gene was named csnE (GenBank accession number AY126455) according to the unified COP9 signalosome nomenclature (Deng et al. 2000).

We substituted the 5 '-terminal part of the csnE coding sequence with the pyr4 marker cassette. The resulting mutant strain AGB209 showed homologous integration of the marker in Southern experiments and no csnE transcripts in Northern hybridisation analysis. Similarly to the $\operatorname{csn} D$ deletion strain, the $\operatorname{csn} E$ mutant was acleistothecial but developed Hülle cells and primordia (Fig. 4.5). This strain also produced highly branched and red hyphae when grown at an airinterface (not shown). All mutant phenotypes were abolished in strain AGB211 where a genomic wild-type copy of $c s n E$ was ectopically integrated in the deletion strain. (Fig. 4.6). Thus, absence of either subunit 4 or subunit 5 of the COP9 signalosome in A. nidulans mediated identical pleiotrophic mutant phenotypes. This indicates that both, CSND and CSNE, are involved in the same function that is probably similar to the function of the COP9 signalosome in higher eukaryotes. 


\subsubsection{The csnE and $c s n D$ gene products of $A$. nidulans interact in a two-hybrid assay.}

All subunits of the COP9 signalosome contain either a PCI or a MPN domain that probably confer to protein-protein interactions (Kapelari et al. 2000). The corresponding regions were identified in silico from the deduced CSND and CSNE peptide sequences (Fig. 4.2). Protein interactions for the A. nidulans proposed CSN subunits were analysed in the yeast two-hybrid system in which interaction of two test partners activates transcription of the $L E U$ and lacZ genes in the yeast twohybrid reporter strain EGY48-p1840 (MAT $\alpha$, his3, trp1, ura3-52, leu2::pLEU2LexAop6, URA3::/acZ-LexAop2). Correspondingly, growth and expression of the $\beta$ galactosidase reporter were analysed on yeast SC medium with galactose and raffinose as carbon source and lacking leucine. $\operatorname{csn} D$ was cloned as fusion to both the LexA DNA binding-domain and the transcriptional activation domain. csnE was fused to the transcriptional activation domain only, since fusion of the corresponding mammalian CSN5 orthologue to the GAL1p binding domain showed strong background activity (Nordgard et al. 2001). CSND and CSNE fusion proteins were combined with the empty two hybrid vectors and with each other in the $S$. cerevisiae reporter strain. Both CSND fusions showed no background activity whereas the CSNE fusion protein showed slight intrinsic transcriptional activity. We identified protein-protein interaction between CSND and CSNE as well as selfassociation of CSND (Fig. 4.7). Both are in agreement with findings of subunitsubunit interactions of the COP9 signalosome in other organisms (Kapelari et al. 2000; Tsuge et al. 2001). We thus assume that CSND and CSNE function within a protein complex and the phenotypic effects discussed in this work resulted from malfunction of this complex in A. nidulans. All our data suggest that this protein complex is the fungal equivalent of the COP9 signalosome of higher eukaryotes.

\subsubsection{Light-dependence of development is disturbed in the $\operatorname{csn} D$ deletion strain.}

Our results suggest that the COP9 signalosome of $A$. nidulans is involved in molecular control of several physiological and morphological processes. We further questioned whether the regulatory role of this complex includes integration of environmental signals. In A. nidulans, knowledge about developmental response to physical parameters on the molecular level is scarce. One important parameter for initiation of development in A. nidulans is illumination: Light generally induces asexual development and represses sexual development. In the plant Arabidopsis thaliana, the COP9 signalosome was initially described as a regulator of light-induced development (Wei et al. 1994). We were thus interested in the developmental response of the $A$. nidulans $\operatorname{csn} D$ deletion strain to light induction. The only component of a proposed light-regulatory pathway in A. nidulans is the veA gene product (Mooney and Yager 1990). VEA (GenBank accession number AF109316) was described as mediator of light-signalling, though the molecular function of this regulatory protein is still obscure. Overexpression of veA in strain OVAR5 mediates increased cleistothecial development and low conidiophore 


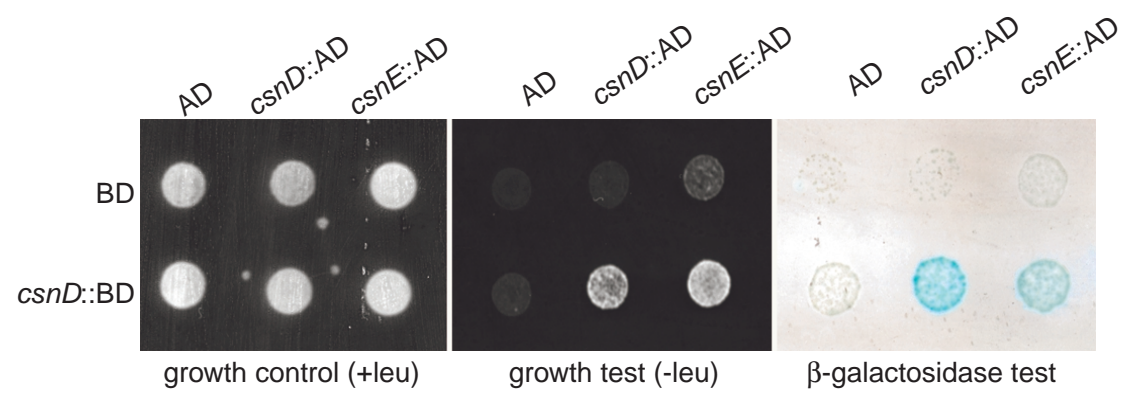

Fig. 4.7: A. nidulans CSND interacts with CSNE in a yeast two-hybrid assay. csnD was fused to the LexA DNA-binding domain (BD) and the yeast GAL1p transcriptional activation domain $(A D)$, respectively, and tested in combination with itself and csnE fused to the $A D$ domain. Protein-protein interaction between the two test partners allows growth of the leuauxotrophic $S$ cerevisiae strain EGY48-p1840 in the absence of leucine in the medium due to the LexA-DNA binding site in the deficient LEU2 promoter. In addition, the heterologous lacZ gene is activated by a functional hybrid.

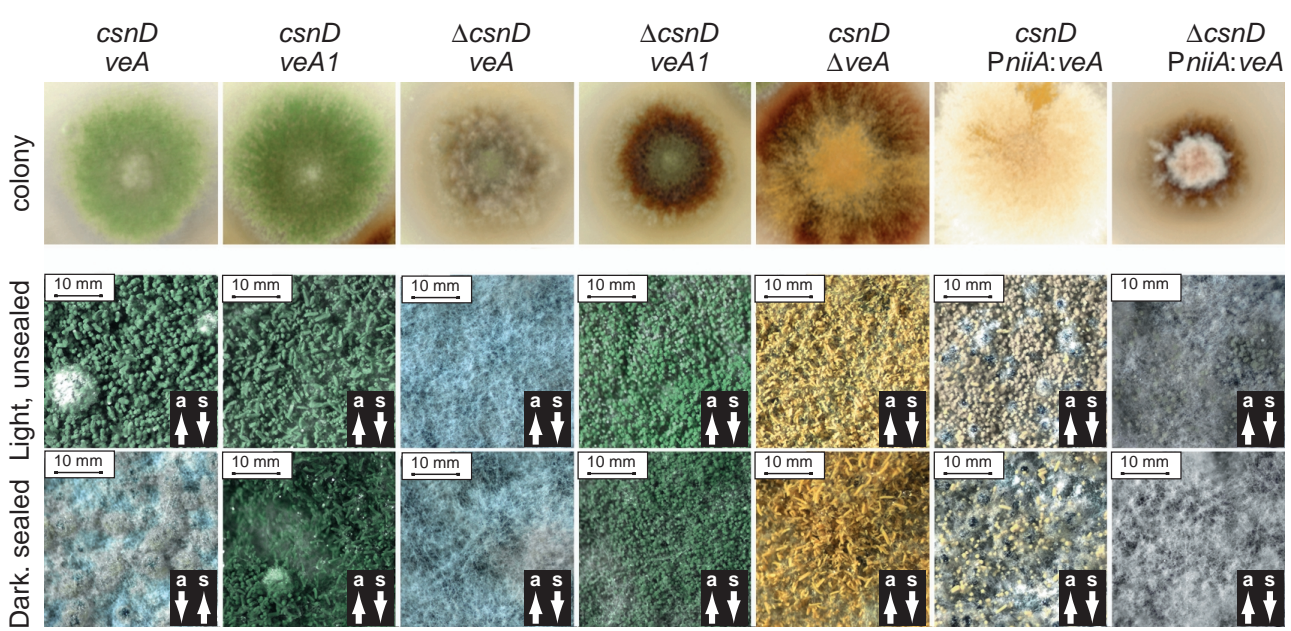

Fig. 4.8: The COP9 signalosome of $\boldsymbol{A}$. nidulans is involved in light-dependent initiation of development. The $\operatorname{csn} D$ deletion was combined with the truncated veA1 allele (strain $\mathrm{AGB192)}$ and a $v e A$ overexpression (AGB220). As controls, the $A$. nidulans wild-type strain AGB160, the csnD deletion strain AGB195, the veA1 mutant strain AGB162, the veA deletion strain DVAR 1 and the veA overexpression strain OVAR5 are shown. The veA overexpression is driven by the $A$. nidulans PniiA promoter which is repressed by weak nitrogen sources. Notably, this promoter may become repressed on older plates when nitrogen sources become exhausted. The overall phenotype of the strains on solid medium incubated in the light is shown in photographs of the colonies (upper row). The $\operatorname{csn} D$ deletion strains produced conidiospores predominantly in the centre of the colony. The veA deletion strain produced exclusively conidiophores and the $v e A$ overexpression strain predominantly cleistothecia (few conidiophores show up in the $v e A$ overproduction strain at longer incubation time). Red colouring beneath the surface material was evident in all $\operatorname{csn} D$ deletion strains and in the $\Delta v e A$ mutant. Light response of development was analysed with pre-grown, developmentally competent mycelia that were transferred to solid medium and induced either asexually in the light (middle row) or sexually on sealed plates in the dark (lower row). Pictures were taken under the binocular. Occurrence of any sexual tissue types was valued as induced sexual reproduction cycle. Induction or repression of asexual (a) or the sexual (s) reproduction is indicated by arrows. 
production. In contrast to that, the veA deletion strain DVAR1 produces conidiophores but is acleistothecial, additionally producing a red hyphal pigment reminiscent to the one observed in the csnD deletion strains (Fig. 4.8). Notably, most frequently used $A$. nidulans laboratory strains carry the veA1 allele (GenBank accession number AF109317), a partial deletion of the open reading frame, which abolishes light-dependence of conidiation and reduces production of cleistothecia and thus resembles a weak veA deletion phenotype (Champe et al. 1994).

Strains with alterations in the $c s n D$ and veA locus were constructed. The $\operatorname{csn} D$ gene was deleted in the $A$. nidulans veA1 background of strain AGB162, resulting after backcross in the $c s n D$ deletion strain AGB192. In $A$. nidulans strain AGB220, the csnD deletion was combined with the PniiA-driven veA overexpression of strain OVAR5. To analyse light-dependence of development, we concentrated on the initiation of the two reproduction pathways in developmentally synchronised, competent mycelia transferred to agar plates. The differential induction of the two reproductive cycles is generally not absolute, and for clarity, occurrence of less than about $25 \%$ conidiophores or cleistothecia compared to the wild-type situation is not taken into account. An $A$. nidulans wild-type strain predominantly induced the sexual cycle in the dark and the asexual cycle in the light (Fig. 4.8). In the strains with altered cellular VEA levels or the veA1 mutant allele, illumination made no difference: the veA1 partial deletion and $v e A$ knock-out strains predominantly induced the asexual cycle whereas veA overexpression resulted in sexual induction. Comparably, deletion of $c s n D$ in wild-type background (AGB195) predominantly caused induction of the sexual cycle independent of the light signal. Thus, a deletion of $c s n D$ abolishes light dependence of development in $A$. nidulans indicating that the COP9 signalosome of $A$. nidulans is essential for correct light-dependent signalling. Combination of $\triangle c s n D$ with different veA alleles, results in strains lacking light dependence of development. Combined with the veA1 allele, the csnD deletion strain (AGB192) preferentially induces the asexual cycle, like its parent veA1 strain. And in combination with $v e A$ overproduction, a $\operatorname{csn} D$ deletion results in induction of the sexual cycle (AGB220), like a pure $\Delta c s n D$ or veA overproduction strain. Thus, with respect to developmental induction, the $\operatorname{csn} D$ deletion did not change the veA mutant phenotypes. This indicates an additional function of the COP9 signalosome in $A$. nidulans in a light-dependent signalling pathway that acts genetically upstream or at the level of VEA - or in a veAindependent pathway.

\subsubsection{The velvet gene product does not release the sexual block of $\operatorname{csn} D$ deletion strains}

We investigated a molecular connection between $c s n D$ and veA. A dependency of $c s n D$ and $v e A$ transcripts was analysed in Northern hybridisation experiments. Wild-type, $\operatorname{csn} D$ deletion and veA1 strains were grown in liquid overnight culture for $18 \mathrm{~h}$ to synchronise the mycelia at the stage of developmental competence. This cell material was transferred to solid medium and induced asexually or sexually in light or in the dark on sealed plates. On the transcriptional level (Fig. 4.9), specific 


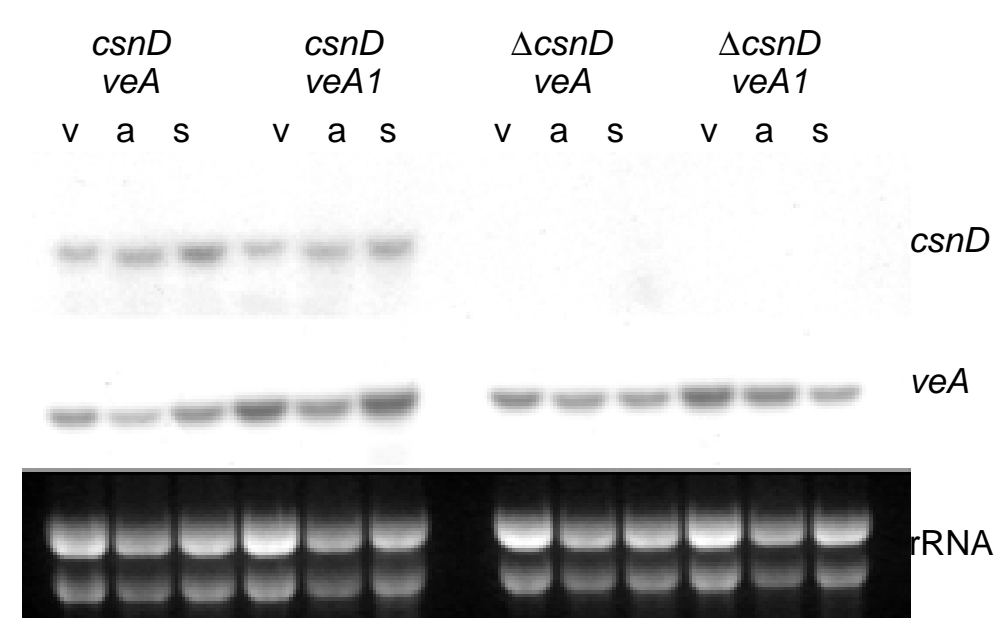

Fig. 4.9: Transcription of $\operatorname{csn} D$ and veA during development. $A$. nidulans strains $A G B 160$ $(c s n D, v e A), A G B 162$ (csnD, veA1), AGB195 ( $\triangle c s n D$, veA) and AGB192 ( $\Delta c s n D, v e A 1)$ were pre-grown in submerged liquid culture, the developmentally competent mycelia were transferred to solid medium and induced either asexually in the light or sexually in the dark on sealed plates. Vegetative (v), asexual (a) and sexual (s) tissue types were harvested for RNA isolation for Northern hybridisation experiments using $c s n D$ and $v e A$ as specific probes. Levels of specific $c s n D$ and $v e A$ mRNAs were independent of the strain and the developmental conditions.

csnD and veA RNA signals from this cell material, compared to rRNA, were not significantly altered after shift from vegetative to differentiating cultures in the wildtype strain and the veA1 strain. Apparently, the mean veA transcript levels were generally higher in the veA1 strain. $c s n D$ transcripts were present independent of the veA allelic state. Vice versa, the quantity of $v e A$ transcript was similar in $c s n D$ wild-type, deletion and overexpression strains. Taken together, transcription of $\operatorname{csn} D$ and $v e A$ proceeds independently, irrespective of the developmental state of the culture.

The phenotypic consequence of the veA1 deletion and the overproduction of the veA wild-type gene in a $\operatorname{csn} D$ deletion strain was analysed to test their genetic relationship. The $\triangle c s n D / v e A 1$ mutant (AGB192) showed both red pigmentation and aberrant cell forms like the $c s n D / v e A+$ strain (AGB195). AGB192 also produced Hülle cells and primordia that never matured to cleistothecia, though apparently less than the corresponding $c s n D / v e A+$ strain. (Fig. 4.8). Thus, the veA1 mutant is unable to suppress the block in development caused by $\operatorname{csn} D$ deletion. All phenotypes of the deletion mutant AGB192 were complemented by ectopic integration of the corresponding $c s n D$ genomic fragment in strain AGB193. The A. nidulans $\triangle c s n D /$ PniiA:veA strain (AGB220) showed red colouring, highly branched hyphae with aberrant cells were readily visible in this mutant, and also sexual development was blocked at the level of cleistothecial primordia (Fig. 4.8). This indicates that even overproduction of VEA, which normally leads to enhanced cleistothecia production, does not lead to development of mature cleistothecia in a $\operatorname{csn} D$ deletion strain. Thus, neither high VEA levels nor the changed veA gene product are able to overrule the developmental block in $\operatorname{csn} D$ deletion strains. Consequently, $c s n D$ is epistatic to the veA1 loss of function and veA gain of function mutations, which places this function of CSND in respect to specific sexual development genetically downstream of VEA. 


\subsection{Discussion}

This study identifies the existence of the COP9 signalosome in filamentous fungi and describes it as key regulator of fungal development. The $\operatorname{csn} D$ and $\operatorname{csn} E$ genes encode the fourth and fifth CSN subunits of $A$. nidulans. The deduced peptide sequences for CSND and CSNE contain PCI and MPN motifs, respectively, which are characteristic for proteins of the $26 \mathrm{~S}$ proteasome lid, elF3 and the COP9 signalosome multiprotein complexes (Kim et al. 2001). Protein-protein interactions between CSND and CSNE are comparable to that described for subunits 4 and 5 of the COP9 signalosome in other organisms (Kapelari et al. 2000; Tsuge et al. 2001). At the transcriptional level, $c s n D$ mRNAs are abundant in both vegetative and developing cultures. In analogy to this, specific mRNAs of CSN subunits were detected in all mouse embryonic and adult tissue tested (Bounpheng et al. 2000). A fusion of CSND to the green fluorescent protein is dispersed in the cytoplasm and clearly enriched in the nuclei. This is in agreement with observations in other organisms, where subunits of the CSN are predominantly localised in the nucleus as multiprotein complex, and subunits 4 to 8 were additionally found in the cytoplasm probably forming a smaller subcomplex (Chamovitz et al. 1996; Kwok et al. 1998; Tsuge et al. 2001; Tomoda et al. 2002). All our data suggest that the products of the two identified genes represent the first members of the COP 9 signalosome in filamentous fungi.

In higher eukaryotes, defects in subunits of the COP9 signalosome result in severe developmental phenotypes and post-embryonic lethality (Wei et al. 1994; Freilich et al. 1999), whereas malfunction of the complex in S. pombe is not lethal and leads to minor mutant phenotypes like delayed progression through the cell cycle and increased sensitivity to ultraviolet light (Mundt et al. 1999; Mundt et al. 2002). This work identified the COP9 signalosome of $A$. nidulans as a key regulator in the development of the organism, essential for proper regulation of metabolism, cell morphology, hyphal polarity, light-regulation and sexual reproduction (Fig. 4.10). The study of the COP9 signalosome in the model organism A. nidulans has three major advantages: it is easily accessible to molecular manipulations. In contrast to COP9 signalosome defects in higher eukaryotes, an A. nidulans strain defective in its sexual cycle is still viable and can propagate via its asexual cycle. Last but not least, $A$. nidulans is evolutionary closer related to humans than plants are.

Malfunction of the COP9 signalosome in $A$. nidulans results in changes of secondary metabolism which is visible with the naked eye: overproduction of a red pigment. To date, we have no indication about the origin of this red substance. In A. nidulans, knowledge about regulation of secondary metabolism, and especially about red pigments, is rather restricted. Wild-type strains deposit brownish melanin in walls of older hyphae when mycelia are grown in submerged liquid culture (Pirt and Rowley 1969). This phenomenon is not altered in the csn deletion mutants compared to the wild-type (not shown). Notably, absence of melanin is correlated with defective sexual reproduction in A. nidulans, indicating a crossconnection between secondary metabolism and sexual development (Champe et 


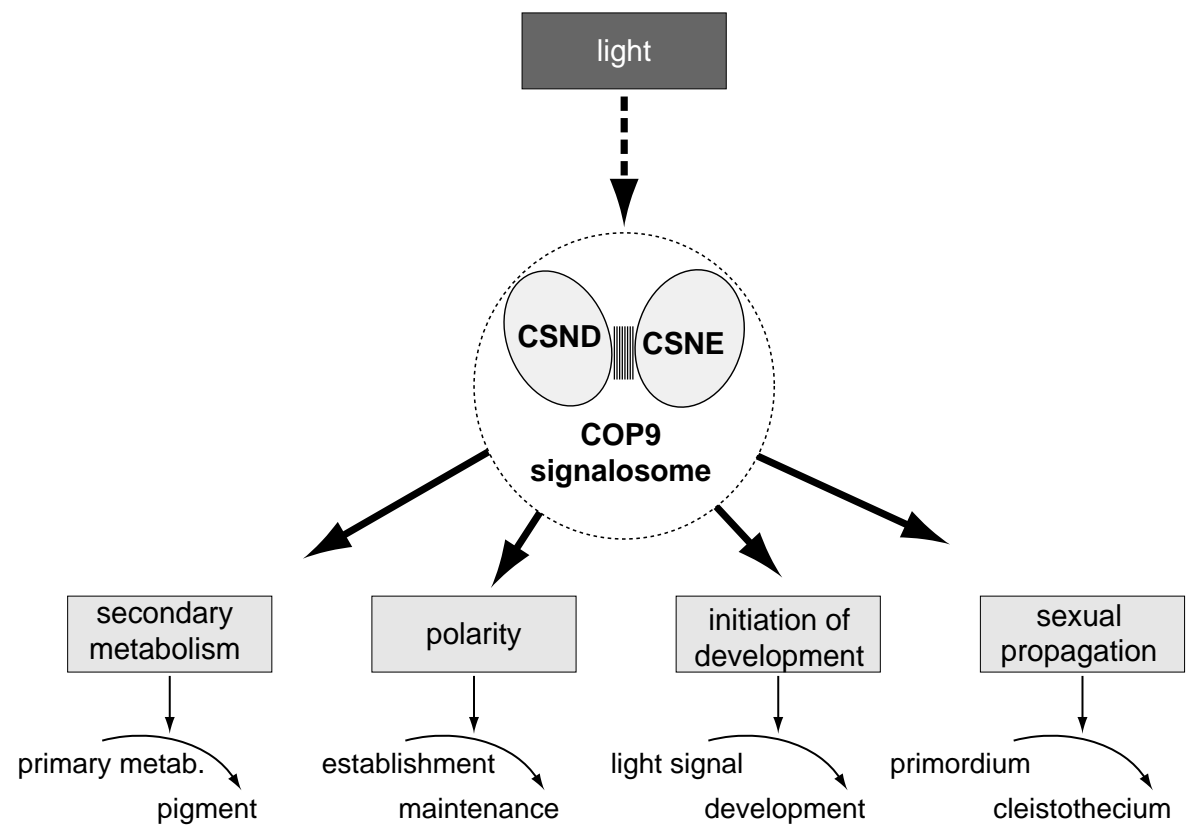

Fig. 4.10: Multifaceted role of the COP9 signalosome (CSN) in A. nidulans. Protein-protein interaction between the two CSN subunits CSND and CSNE are indicated by paralleled bars. The impact of the COP9 signalosome in a lightdependent signalling pathway is indicated by a broken arrow line and the function of the CSN in several downstream target pathways by full arrow lines.

al. 1994). The second known red pigment produced by $A$. nidulans is responsible for the colour of the ascospores: the anthraquinone asperthecin, which is difficult to isolate (Howard and Raistrick 1955). It seems striking that the red colouring in the $\operatorname{csn} D$ mutant becomes visible after two-three days of growth which is the time scale where also the first structures of sexual development become visible. Notably, CSN mutants of the plant $A$. thaliana overproduce anthocyan, the flowering colour (Misera et al. 1994). This raises the question whether the red pigment produced in the csn deletion strains is related to the one that dyes the ascospores. Preliminary tests using $0.5 \mathrm{M} \mathrm{NaHCO}_{3}$ and $\mathrm{Na}_{2} \mathrm{CO}_{3}$ according to Howard and Raistrick (1955) did not reveal whether the red colour of the csn deletion mutants was asperthecin (data not shown). The aberrant colouring of hyphae in the csn deletion strains appears independent of developmental induction by light and the allelic veA state. Regulation of the production of the putative red pigment thus seems to be mainly mediated by time and/or growth phase. Thus, an impact of the COP9 signalosome in the internal regulation of onset of secondary metabolism and/or development is conceivable. Astonishingly, red coloured hyphae are also seen in a veA deletion strain, but it is not clear whether the red pigment produced by $\Delta c s n$ and $\Delta v e A$ strains is identical.

The COP9 signalosome of $A$. nidulans is involved in the control of polar apical growth and lateral branching in surface-grown cultures. Generally, establishment of polar growth after germination and its later maintenance seem independent processes in A. nidulans, with a proposed persistent signal for ongoing apical extension (Momany et al. 1999). Young hyphae of surface-grown csn deletion colonies as well as hyphae grown in submerged liquid culture show 
no obvious aberrance. Thus, the establishment of polarity seems not generally disturbed in the csn deletion strains. But maintenance of apical extension in elderly, surface-grown cell material seems to be a target of the COP9 signalosome. In A. nidulans wild-type strains, polarised apical growth is generally turned off during developmental programs. In the asexual propagation cycle, a switch from polarised growth to a bud-like growth form is seen during sterigmata formation and conidiation (Adams et al. 1998). As for propagation of sexual spores, ascogenous hyphae are formed that can be seen in young immature cleistothecia as branched filaments with knobby cells (Braus et al. 2002). A. nidulans strains with overproduction of the transcription factor STEA block vegetative growth and produce highly branched hyphae with small, knobby cells very similar to ascogenous tissue, though a direct relation has not been proven yet (Vallim et al. 2000). Nevertheless, the short and highly branched hyphae in elderly csn deletion strains do not morphologically resemble the phenotype described for the STEA overproduction strain and young ascogenous tissue. Similar to the polarity defect of $A$. nidulans csn deletion strains, malfunction of the $A$. thaliana COP9 signalosome causes aberrant cell morphologies. Transgenic plants with reduced CSN levels show a general increase in secondary inflorescences and a reduction of internode length and cell size. These phenomena are primarily due to a loss of apical dominance, which in turn is driven by the phytohormone auxin. In $A$. thaliana, the auxinresponse is controlled by the COP9 signalosome, probably by degradation of the AUXIIAA transcriptional repressors (Schwechheimer et al. 2001). Auxin, a tryptophan-related hormone-like signal molecule, is product of secondary metabolism. We have recently reported a role of auxin for development in A. nidulans (Eckert et al. 2000). Strains auxotrophic for tryptophan arrest sexual development at the level of micro-cleistothecia, which is one step beyond the arrest at primordia seen in csn deletion strains. External supply of high amounts of tryptophan or traces of auxin released this developmental block. Future studies will focus on a possible co-ordination of sexual development and hormone signalling by the $A$. nidulans COP9 signalosome.

The severe mutant phenotype of $\operatorname{csn} D$ or $\operatorname{csn} E$ deletion in $A$. nidulans is a block in sexual development at the level of cleistothecial primordia. To our knowledge, a specific developmental arrest at this stage was not described before in $A$. nidulans. Initiation of the sexual cycle and differentiation processes leading to the general architecture of primordia are not impaired in the csn deletion strains. But further differentiation and maturation of wall and ascospores is blocked. This suggests that after successful formation of the primordial structure, a regulatory process exists that is expendable for the first steps in sexual development leading to cleistothecial primordia but essential for completion of the sexual cycle. The COP9 signalosome seems to be an essential player in this regulatory process mediating maturation of primordia in $A$. nidulans. A similar developmental block at a level of metamorphosis of a primordial to mature stage can also be observed in homozygous CSN mutants of the fruit fly $D$. melangolaster. The mutant embryos hatch and develop normally until the middle of the third instar and frequently pupate, but then cease to develop and die (Freilich et al. 1999). This block in sexual 
development seems to be the most severe phenotype of a defect in COP9 signalosome function in A. nidulans. And as stated above, the additional phenotypes of cell polarity and red pigmentation may also be related to developmental processes. It is thus conceivable that the COP9 signalosome in A. nidulans is dispensible for growth and housekeeping functions but essential for correct regulation of development.

Due to its impact on secondary metabolism, polarity and sexual development, the COP9 signalosome probably has several different downstream targets, summarised in Figure 4.10. This raises the question which upstream factors regulate CSN activity. An external signal important for development in A. nidulans is light, with the veA gene product as a proposed part of a corresponding signal transduction pathway. A $\operatorname{csn} D$ deletion strain is "blind" to light-regulation, like strains with constitutively low or high veA expression. Thus, the COP9 signalosome of $A$. nidulans is involved in light-dependent signalling and may even be connected with the same signal transduction pathway as VEA. Notably, in the plant A. thaliana, the CSN is involved in the repression of photomorphogenesis in the dark. The proposed E3 ubiquitin ligase COP1 accumulates in the nucleus in the dark where it mediates, assisted among others by the COP9 signalosome, ubiquitinylation of an transcriptional activator of lightregulated genes (Osterlund et al. 1999; Osterlund et al. 2000; Schwechheimer and Deng 2001; Suzuki et al. 2002). The product of the A. nidulans veA gene has a negative influence on initiation on the asexual but a positive on the onset of the sexual cycle, as seen by the corresponding deletion and overproduction strains. It seems striking that the $c s n D$ deletion strain in a velvet wild-type background acts like a veA overproduction strain: a constitutive induction of the sexual cycle. In analogy to the findings in $A$. thaliana, it is thus conceivable that the COP9 signalosome of $A$. nidulans mediates a negative post-transcriptional effect on VEA, resulting in increased VEA protein levels in a $\operatorname{csn} D$ deletion strain. The function of the COP9 signalosome in light signalling might thus be genetically placed upstream or at the level of VEA, though this question should be addressed in future studies.

In summary, we present the first report of components of the COP9 signalosome in filamentous fungi and present strong evidence of its key regulatory function of development of the mold Aspergillus nidulans. The COP9 signalosome of $A$. nidulans is involved in several cellular processes including pigment synthesis, cell morphology, light-dependent signalling and specific sexual development. The function of the COP9 signalosome in filamentous fungi resembles in some respects that of higher eukaryotes. Because mutant strains are viable and can be propagated, this study represents an attractive basis to deliver new insights of the functions of the COP9 signalosome in eukaryotes. 


\subsection{References}

Adams, T.H., J.K. Wieser, and J.H. Yu. 1998. Asexual sporulation in Aspergillus nidulans. Microbiol Mol Biol Rev 62: 35-54.

Altschul, S.F., W. Gish, W. Miller, E.W. Myers, and D.J. Lipman. 1990. Basic local alignment search tool. J Mol Biol 215: 403-410.

Axelrod, D.E., M. Gealt, and M. Pastushok. 1973. Gene control of developmental competence in Aspergillus nidulans. Dev Biol 34: 9-15.

Bech-Otschir, D., R. Kraft, X. Huang, P. Henklein, B. Kapelari, C. Pollmann, and W. Dubiel. 2001. COP9 signalosome-specific phosphorylation targets p53 to degradation by the ubiquitin system. EMBO $J$ 20: $1630-1639$.

Bech-Otschir, D., M. Seeger, and W. Dubiel. 2002. The COP9 signalosome: at the interface between signal transduction and ubiquitin-dependent proteolysis. J Cell Sci 115: 467-473.

Bennett, J.W. and L.L. Lasure. 1991. Growth media. In More gene manipulation in fungi (ed. J.W. Bennett and L.L. Lasure), pp. 441-457. Academic Press Inc, San Diego.

Bounpheng, M.A., I.N. Melnikova, S.G. Dodds, H. Chen, N.G. Copeland, D.J. Gilbert, N.A. Jenkins, and B.A. Christy. 2000. Characterization of the mouse JAB1 CDNA and protein. Gene 242: 41-50.

Braus, G.H., S. Krappmann, and S.E. Eckert. 2002. Sexual development in ascomycetes: Fruit body formation of Aspergillus nidulans. In Molecular Biology of Fungal Development (ed. H.D. Osiewacz), pp. 215-244. Marcel Dekker, Inc., New Yok, Basel.

Busby, T.M., K.Y. Miller, and B.L. Miller. 1996. Suppression and enhancement of the Aspergillus nidulans medusa mutation by altered dosage of the bristle and stunted genes. Genetics 143: 155-163.

Busch, S., B. Hoffmann, O. Valerius, K. Starke, K. Duvel, and G.H. Braus. 2001. Regulation of the Aspergillus nidulans hisB gene by histidine starvation. Curr Genet 38: 314-22.

Bussink, H.J. and S.A. Osmani. 1998. A cyclin-dependent kinase family member (PHOA) is required to link developmental fate to environmental conditions in Aspergillus nidulans. EMBO J 17: 3990-4003.

Chamovitz, D.A., N. Wei, M.T. Osterlund, A.G. von Arnim, J.M. Staub, M. Matsui, and X.W. Deng. 1996. The COP9 complex, a novel multisubunit nuclear regulator involved in light control of a plant developmental switch. Cell 86: 115-121.

Champe, S.P. and A.A. el-Zayat. 1989. Isolation of a sexual sporulation hormone from Aspergillus nidulans. $J$ Bacteriol 171: 3982-3988.

Champe, S.P., D.L. Nagle, and L.N. Yager. 1994. Sexual sporulation. In Aspergillus: 50 years on. Prog Ind Microbiol (ed. S.D. Martinelli and J.R. Klinghorn), pp. 429-454. Elsevier, Amsterdam.

Champe, S.P., P. Rao, and A. Chang. 1987. An endogenous inducer of sexual development in Aspergillus nidulans. J Gen Microbiol 133: 1383-1387.

Clutterbuck, A.J. 1969. A mutational analysis of conidial development in Aspergillus nidulans. Genetics 63: 317-327.

Clutterbuck, A.J. 1974. Aspergillus nidulans. In Handbook of Genetics. (ed. R.C. King), pp. 447-510. Plenum, New York.

Corpet, F. 1988. Multiple sequence alignment with hierarchical clustering. Nucleic Acids Res 16: 10881-10890.

Deng, X.W., W. Dubiel, N. Wei, K. Hofmann, K. Mundt, J. Colicelli, J. Kato, M. Naumann, D. Segal, M. Seeger, A. Carr, M. Glickman, and D.A. Chamovitz. 2000. Unified nomenclature for the COP9 signalosome and its subunits: an essential regulator of development. Trends Genet 16: 202-203.

Eckert, S.E., B. Hoffmann, C. Wanke, and G.H. Braus. 1999. Sexual development of Aspergillus nidulans in tryptophan auxotrophic strains. Arch Microbiol 172: 157-166. ny.com/link/service/journals/00203/bibs/172n3p157.html.

Eckert, S.E., E. Kubler, B. Hoffmann, and G.H. Braus. 2000. The tryptophan synthase-encoding $\operatorname{trp} B$ gene of Aspergillus nidulans is regulated by the cross-pathway control system. Mol Gen Genet 263: 867-876.

Elble, R. 1992. A simple and efficient procedure for transformation of yeasts. Biotechniques 13: 18-20.

Fernandez-Abalos, J.M., H. Fox, C. Pitt, B. Wells, and J.H. Doonan. 1998. Plant-adapted green fluorescent protein is a versatile vital reporter for gene expression, protein localization and mitosis in the filamentous fungus, Aspergillus nidulans. Mol Microbiol 27: 121-130.

Freilich, S., E. Oron, Y. Kapp, Y. Nevo-Caspi, S. Orgad, D. Segal, and D.A. Chamovitz. 1999. The COP9 signalosome is essential for development of Drosophila melanogaster. Curr Biol 9: 1187-1190.

Glickman, M.H., D.M. Rubin, O. Coux, I. Wefes, G. Pfeifer, Z. Cjeka, W. Baumeister, V.A. Fried, and D. Finley. 1998. A subcomplex of the proteasome regulatory particle required for ubiquitin-conjugate degradation and related to the COP9-signalosome and elF3. Cell 94: 615-623.

Golemis and R. Brent. 1996. Protein interaction studies. In Current protocols in molecular biology (ed. F.M. Ausubel, R. Brent, R.E. Kingston, D.D. Moore, J.G. Seidmann, A.J. Smith, and K. Struhl), pp. 429454. Harvard Medical School, Massachusetts. 
Guthrie, C. and G.R. Fink. 1991. Guide to yeast genetics and molecular biology. Methods Enzymol. $194: 15$.

Gyuris, J., E. Golemis, H. Chertkov, and R. Brent. 1993. Cdi1, a human G1 and S phase protein phosphatase that associates with Cdk2. Cell 75: 791-803.

Han, D.M., Y.J. Han, K.S. Chae, K.Y. Jahng, and Y.H. Lee. 1994. Effects of various carbon sources on the development of Aspergillus nidulans with velA+ or velA1 allele. Korean. J. Mycol. 22: 332-337.

Han, K.H., K.Y. Han, J.H. Yu, K.S. Chae, K.Y. Jahng, and D.M. Han. 2001. The nsdD gene encodes a putative GATA-type transcription factor necessary for sexual development of Aspergillus nidulans. Mol Microbiol 41: 299-309.

Hermann, T.E., M.B. Kurtz, and S.P. Champe. 1983. Laccase localized in hulle cells and cleistothecial primordia of Aspergillus nidulans. J Bacteriol 154: 955-964.

Hoffmann, B., C. Wanke, S.K. Lapaglia, and G.H. Braus. 2000. c-Jun and RACK1 homologues regulate a control point for sexual development in Aspergillus nidulans. Mol Microbiol 37: 28-41.

Howard, B.H. and H. Raistrick. 1955. The colouring matters of species in the Aspergillus nidulans group. Biochem. J. 59: 476-484.

Inoue, H., H. Nojima, and H. Okayama. 1990. High efficiency transformation of Escherichia coli with plasmids. Gene 96: 23-28.

Käfer, E. 1977. Meiotic and mitotic recombination in Aspergillus and its chromosomal aberrations. Adv Genet 19: 33-131.

Kapelari, B., D. Bech-Otschir, R. Hegerl, R. Schade, R. Dumdey, and W. Dubiel. 2000. Electron microscopy and subunit-subunit interaction studies reveal a first architecture of COP9 signalosome. J Mol Biol 300: 1169-1178.

Kim, T., K. Hofmann, A.G. von Arnim, and D.A. Chamovitz. 2001. PCl complexes: pretty complex interactions in diverse signaling pathways. Trends Plant Sci 6: 379-386.

Kirk, K.E. and N.R. Morris. 1991. The tubB alpha-tubulin gene is essential for sexual development in Aspergillus nidulans. Genes Dev 5: 2014-2023.

Kwok, S.F., R. Solano, T. Tsuge, D.A. Chamovitz, J.R. Ecker, M. Matsui, and X.W. Deng. 1998. Arabidopsis homologs of a c-Jun coactivator are present both in monomeric form and in the COP9 complex, and their abundance is differentially affected by the pleiotropic cop/det/fus mutations. Plant Cell 10: $1779-1790$

Lee, B.S. and J.W. Taylor. 1990. Isolation of DNA from fungal mycelia and single spores. In PCR protocols: $A$ guide to methods and applications (ed. M.A. Innis, D.H. Gelfand, J.S. Sninsky, and T.J. White), pp. 282-287. Academic Press Inc, San Diego.

Lewis, C. and S.P. Champe. 1995. A pre-induction sporulation gene from Aspergillus nidulans. Microbiology 141: 1821-1828.

$\mathrm{Li}$, S., X. Liu, and M. Ascoli. 2000. p38JAB1 binds to the intracellular precursor of the lutropin/choriogonadotropin receptor and promotes its degradation. J Biol Chem 275: 13386-13393.

Lyapina, S., G. Cope, A. Shevchenko, G. Serino, T. Tsuge, C. Zhou, D.A. Wolf, N. Wei, and R.J. Deshaies. 2001. Promotion of NEDD-CUL1 conjugate cleavage by COP9 signalosome. Science 292: 1382-1385.

Miller, K.Y., J. Wu, and B.L. Miller. 1992. StuA is required for cell pattern formation in Aspergillus. Genes Dev 6: $1770-82$.

Misera, S., A.J. Muller, U. Weiland-Heidecker, and G. Jurgens. 1994. The FUSCA genes of Arabidopsis: negative regulators of light responses. Mol Gen Genet 244: 242-252.

Momany, M., P.J. Westfall, and G. Abramowsky. 1999. Aspergillus nidulans swo mutants show defects in polarity establishment, polarity maintenance and hyphal morphogenesis. Genetics 151: 557-567.

Mooney, J.L. and L.N. Yager. 1990. Light is required for conidiation in Aspergillus nidulans. Genes Dev 4: 1473-1482.

Mundt, K.E., C. Liu, and A.M. Carr. 2002. Deletion mutants in COP9/signalosome subunits in fission yeast Schizosaccharomyces pombe display distinct phenotypes. Mol Biol Cell 13: 493-502.

Mundt, K.E., J. Porte, J.M. Murray, C. Brikos, P.U. Christensen, T. Caspari, I.M. Hagan, J.B. Millar, V. Simanis, K. Hofmann, and A.M. Carr. 1999. The COP9/signalosome complex is conserved in fission yeast and has a role in S phase. Curr Bio/ 9: 1427-1430.

Nordgard, O., O. Dahle, T.O. Andersen, and O.S. Gabrielsen. 2001. JAB1/CSN5 interacts with the GAL4 DNA binding domain: a note of caution about two-hybrid interactions. Biochimie 83: 969-971.

Ohh, M., W.Y. Kim, J.J. Moslehi, Y. Chen, V. Chau, M.A. Read, and W.G. Kaelin, Jr. 2002. An intact NEDD8 pathway is required for Cullin-dependent ubiquitylation in mammalian cells. EMBO Rep 3: 177-182.

Osterlund, M.T., L.H. Ang, and X.W. Deng. 1999. The role of COP1 in repression of Arabidopsis photomorphogenic development. Trends Cell Biol 9: 113-118.

Osterlund, M.T., C.S. Hardtke, N. Wei, and X.W. Deng. 2000. Targeted destabilization of HY5 during lightregulated development of Arabidopsis. Nature 405: 462-466.

Pirt, S.J. and B.I. Rowley. 1969. Melanin production in Aspergillus nidulans. Biochem J 114: 9P-10P. 
Pollmann, C., X. Huang, J. Mall, D. Bech-Otschir, M. Naumann, and W. Dubiel. 2001. The constitutive photomorphogenesis 9 signalosome directs vascular endothelial growth factor production in tumor cells. Cancer Res 61: 8416-8421.

Punt, P.J., M.A. Dingemanse, A. Kuyvenhoven, R.D. Soede, P.H. Pouwels, and C.A. van den Hondel. 1990. Functional elements in the promoter region of the Aspergillus nidulans gpdA gene encoding glyceraldehyde-3-phosphate dehydrogenase. Gene 93: 101-109.

Punt, P.J. and C.A. van den Hondel. 1992. Transformation of filamentous fungi based on hygromycin B and phleomycin resistance markers. Methods Enzymol 216: 447-457.

Rave, N., R. Crkvenjakov, and H. Boedtker. 1979. Identification of procollagen mRNAs transferred to diazobenzyloxymethyl paper from formaldehyde agarose gels. Nucleic Acids Res 6: 3559-3567.

Sanchez, O., R.E. Navarro, and J. Aguirre. 1998. Increased transformation frequency and tagging of developmental genes in Aspergillus nidulans by restriction enzyme-mediated integration (REMI). $\mathrm{Mol}$ Gen Genet 258: 89-94.

Schiestl, R.H. and T.D. Petes. 1991. Integration of DNA fragments by illegitimate recombination in Saccharomyces cerevisiae. Proc Natl Acad Sci U S A 88: 7585-7589.

Schwechheimer, C. and X. Deng. 2001. COP9 signalosome revisited: a novel mediator of protein degradation. Trends Cell Biol 11: 420-426.

Schwechheimer, C., G. Serino, J. Callis, W.L. Crosby, S. Lyapina, R.J. Deshaies, W.M. Gray, M. Estelle, and X.W. Deng. 2001. Interactions of the COP9 signalosome with the E3 ubiquitin ligase SCFTIRI in mediating auxin response. Science 292: 1379-1382.

Seeger, M., R. Kraft, K. Ferrell, D. Bech-Otschir, R. Dumdey, R. Schade, C. Gordon, M. Naumann, and W. Dubiel. 1998. A novel protein complex involved in signal transduction possessing similarities to $26 \mathrm{~S}$ proteasome subunits. Faseb J 12: 469-478.

Southern, E.M. 1975. Detection of specific sequences among DNA fragments separated by gel electrophoresis. J Mol Biol 98: 503-517.

Suzuki, G., Y. Yanagawa, S.F. Kwok, M. Matsui, and X.W. Deng. 2002. Arabidopsis COP10 is a ubiquitinconjugating enzyme variant that acts together with COP1 and the COP9 signalosome in repressing photomorphogenesis. Genes Dev 16: 554-559.

Swart, K., D. van Heemst, M. Slakhorst, F. Debets, and C. Heyting. 2001. Isolation and characterization of sexual sporulation mutants of Aspergillus nidulans. Fungal Genet Biol 33: 25-35.

Timberlake, W.E. and A.J. Clutterbuck. 1994. Genetic regulation of conidiation. In Aspergillus: 50 years on (ed. S.D. Martinelli and J.R. Klinghorn), pp. 1-851. Elsevier, Amsterdam.

Tomoda, K., Y. Kubota, Y. Arata, S. Mori, M. Maeda, T. Tanaka, M. Yoshida, N. Yoneda-Kato, and J.Y. Kato. 2002. The cytoplasmic shuttling and subsequent degradation of p27Kip1 mediated by Jab1/CSN5 and the COP9 signalosome complex. J Biol Chem 277: 2302-2310.

Tsuge, T., M. Matsui, and N. Wei. 2001. The subunit 1 of the COP9 signalosome suppresses gene expression through its $\mathrm{N}$-terminal domain and incorporates into the complex through the $\mathrm{PCl}$ domain. $\mathrm{J} \mathrm{Mol} \mathrm{Biol}$ 305: 1-9.

Tyers, M. and P. Jorgensen. 2000. Proteolysis and the cell cycle: with this RING I do thee destroy. Curr Opin Genet Dev 10: 54-64.

Vallim, M.A., K.Y. Miller, and B.L. Miller. 2000. Aspergillus SteA (sterile12-like) is a homeodomain-C2/H2-Zn+2 finger transcription factor required for sexual reproduction. Mol Microbiol 36: 290-301.

Vieira, J. and J. Messing. 1982. The pUC plasmids, an M13mp7-derived system for insertion mutagenesis and sequencing with synthetic universal primers. Gene 19: 259-68.

Waring, R.B., G.S. May, and N.R. Morris. 1989. Characterization of an inducible expression system in Aspergillus nidulans using alcA and tubulin-coding genes. Gene 79: 119-130.

Wei, N., D.A. Chamovitz, and X.W. Deng. 1994. Arabidopsis COP9 is a component of a novel signaling complex mediating light control of development. Cell 78: 117-124.

Wei, N. and X.W. Deng. 1999. Making sense of the COP9 signalosome. A regulatory protein complex conserved from Arabidopsis to human. Trends Genet 15: 98-103.

$\mathrm{Wu}$, J. and B.L. Miller. 1997. Aspergillus asexual reproduction and sexual reproduction are differentially affected by transcriptional and translational mechanisms regulating stunted gene expression. Mol Cell Biol 17: 6191-6201.

Yager, L.N., M.B. Kurtz, and S.P. Champe. 1982. Temperature-shift analysis of conidial development in Aspergillus nidulans. Dev Biol 93: 92-103.

Yager, L.N., H.O. Lee, D.L. Nagle, and J.E. Zimmerman. 1998. Analysis of fluG mutations that affect lightdependent conidiation in Aspergillus nidulans. Genetics 149: 1777-1786.

Yang, X., S. Menon, K. Lykke-Andersen, T. Tsuge, X. Di, X. Wang, R.J. Rodriguez-Suarez, H. Zhang, and N. Wei. 2002. The COP9 Signalosome Inhibits p27(kip1) Degradation and Impedes G1-S Phase Progression via Deneddylation of SCF Cul1. Curr Biol 12: 667-672. 
Zhou, C., V. Seibert, R. Geyer, E. Rhee, S. Lyapina, G. Cope, R.J. Deshaies, and D.A. Wolf. 2001. The fission yeast COP9/signalosome is involved in cullin modification by ubiquitin-related Ned8p. BMC Biochem 2: 7. 


\section{Conclusions and outlook}

\subsection{Amino acid biosynthesis and the COP9 signalosome in Aspergillus nidulans}

This study focuses on two molecular networks that mediate 'genetic supervision' in eukaryotes, the cross-pathway control of amino acid biosynthesis (CPC) and the COP9 signalosome-dependent network of development (CSN). In this study, components of both regulation circuits of Aspergillus nidulans have been isolated and characterised. The CPC and the CSN-dependent system are essential for proper response to external signals, amino acids and light, respectively. Additionally, both molecular networks are involved in the regulation of metabolism as well as development (Fig. 5.1). The CPC not only transcriptionally activates specific target genes of primary metabolism but also significantly impacts sexual development (Chapter 2) and penicillin biosynthesis (Chapter 3). Similarly, function of the CSN is not restricted to cleistothecia formation but additionally affects secondary metabolism (Chapter 4 ). The results thus imply that cross-connections between physiology and morphology in A. nidulans are partly mediated by the cross-pathway control and the COP9 signalosome.

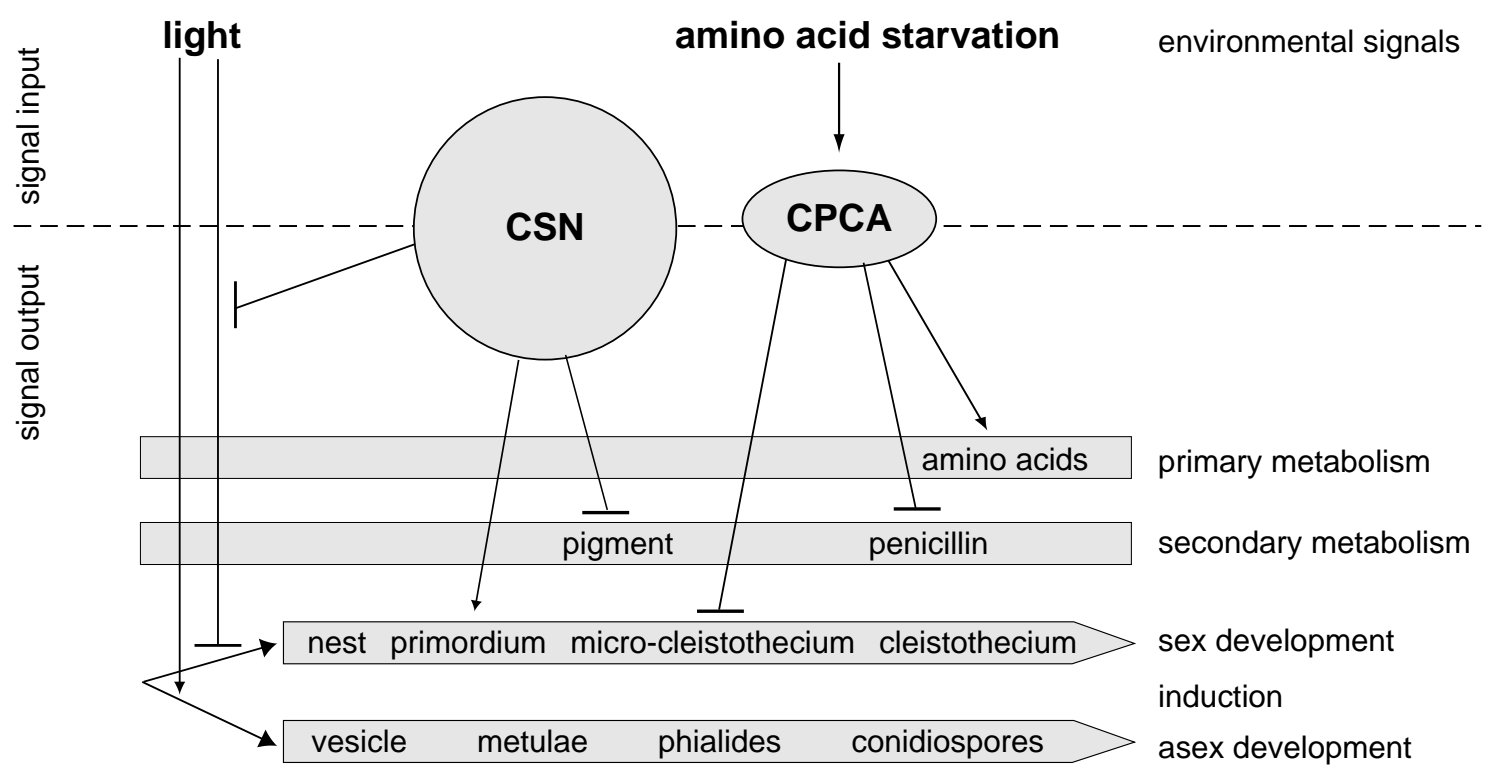

Fig. 5.1: Multifaceted role of cross-pathway control and COP9 signalosome. Both molecular regulators are involved in the control of physiological and metabolic processes in A. nidulans. 


\subsubsection{Signalling of the cross-pathway control}

The impact of the cross-pathway control on physiology as well as morphology implies that various cellular targets are affected. Target selection of this system is thoroughly studied in yeast (see 2.2). It is mediated by the 5'-TGA C/G TCA-3' cisacting nucleotide consensus sequence in the $5^{\prime}$ regulatory region of the target genes, that is recognised by the central transcriptional activator Gcn4p, though aberrations of one or two bases from the consensus can eventually be tolerated (Mavrothalassitis et al. 1990). The A. nidulans CPCA, counterpart of yeast Gcn4p, was recently isolated (Hoffmann et al. 2001). Functional complementation of a yeast GCN4 deletion strain with the A. nidulans cpcA gene (Hoffmann et al. 2001) strongly suggests that in this filamentous fungus the same consensus accounts for transcriptional activation upon amino acid starvation. First evidence for such functional cross-pathway response elements (CPREs) in $A$. nidulans resulted from intensive mutant promoter complementation studies of $\arg B$, the gene encoding ornithine carbamoyltransferase of arginine biosynthesis (Goc and Weglenski 1988). Both CPCA target genes identified in this work, hisB and IysA, contain putative CPREs in the proximal $400 \mathrm{bp}$ of their promoter regions, which is comparable to those identified for other $A$. nidulans CPC-regulated amino acid biosynthesis genes $\arg B$, hisHF and trpB. From this putative CPCA binding sites, the CPRE consensus 5'-TGA C/G TSW-3' can be deduced for A. nidulans, which closely resembles that of the yeast consensus (Tab. 5.1). Strikingly, most of the aberrations from the proposed optimal binding site are located within the $3^{\prime}$ halfsite. An asymmetry of the two halfsites concerning the tolerance of base substitutions from the consensus was also reported from studies on the yeast system, leading to the hypothesis that the two sites are probably not equivalent (Mavrothalassitis et al. 1990).

Tab. 5.1: Proposed CPREs in A. nidulans cross-pathway target genes.

\begin{tabular}{|c|c|c|c|c|c|c|}
\hline gene & regulatory circuit & seque & ence & $\left(5^{\prime}-3^{\prime}\right)$ & pos. ${ }^{1}$ & reference \\
\hline & yeast consensus: & TGA & $\mathrm{C} / \mathrm{G}$ & TCA & & Oliphant et al. 1989 \\
\hline \multirow[t]{3}{*}{$\arg B$} & arginine biosynthesis & TAA & $\mathrm{C}$ & $\mathrm{TCT}$ & -127 & Goc and Weglenski 1988 \\
\hline & & TGA & G & $\mathrm{TCA}$ & -79 & \\
\hline & & TTA & $\mathrm{C}$ & $\mathrm{TAA}^{2}$ & -66 & \\
\hline \multirow[t]{2}{*}{$c p c A$} & cross-pathway control & TCT & $\mathrm{C}$ & $\mathrm{AGT}$ & -1085 & Hoffmann et al. 2001 \\
\hline & & TGA & $\mathrm{C}$ & TCA & -1070 & \\
\hline \multirow{3}{*}{$\begin{array}{l}\text { hisB } \\
\text { hisHF }\end{array}$} & histidine biosynthesis & TGA & $\mathrm{C}$ & GCA & -225 & this work \\
\hline & histidine biosynthesis & TGA & $\mathrm{C}$ & TGT & -396 & Valerius et al. 2001 \\
\hline & & TGA & G & TCA & -154 & \\
\hline \multirow[t]{3}{*}{$j l b A$} & DNA binding protein & TGA & G & TCA & -776 & Strittmatter et al. 2001 \\
\hline & & TGA & $\mathrm{C}$ & TCC & -491 & \\
\hline & & TGA & $\mathrm{C}$ & TCA & -308 & \\
\hline \multirow{2}{*}{$\begin{array}{l}\operatorname{lys} A \\
\operatorname{trp} B\end{array}$} & lysine biosynthesis & TGA & $\mathrm{C}$ & TCA & -174 & this work \\
\hline & tryptophan biosynthesis & TGA & $\mathrm{C}$ & TCA & -182 & Eckert et al. 2000 \\
\hline \multicolumn{2}{|c|}{ deduced Aspergillus consensus ${ }^{3}$ : } & TGA & $\mathrm{C} / \mathrm{G}$ & TSW & & \\
\hline
\end{tabular}


The strong indications about functional conservation between yeast Gcn $4 p$ and $A$. nidulans CPCA suggest an evolutionary conservation of the complete crosspathway system in fungi. The second key player of the corresponding signal transduction cascade is the sensor kinase Gcn2p (Wek et al. 1995) that perceives the external signal 'amino acid starvation' (see 1.2.1.2). A homologue of yeast GCN2 was isolated from the filamentous fungus Neurospora crassa, cpc-3 (Sattlegger et al. 1998). But the corresponding A. nidulans sensor kinase is not yet identified. During this work, functional complementation of a yeast GCN2 deletion strain with an $A$. nidulans cDNA library revealed no positive clones, though this might be due to a lack of the comparably long proposed cDNA (probably about $1.6 \mathrm{~kb}$ ) in the preparation. Also, application of degenerate primer pairs resembling regions conserved between the deduced amino acid sequences of yeast GCN2 and $N$. crassa cpc-3 were not successful. However, completion of the A. nidulans genome sequencing project will probably reveal the cross-pathway sensor kinase of this fungus.

\subsubsection{COP9 signalosome-dependent signalling}

Knowledge about the informational flux from environmental signals to CSNmediated cellular responses is scarce. This work revealed an impact of the COP9 signalosome on light-dependent signalling in A. nidulans (Chapter 4). Two major scenarios seem conceivable: an impact of light on the activity of the COP9 signalosome or an effect of the CSN on a light-dependent regulator. The veA gene product is strongly involved in light-dependent regulation of development (see 1.4.2.1), but this study shows at least on transcriptional level no evidence of a direct interdependency between $v e A$ and $\operatorname{csn} D$. Whether the CSN acts in the same light-dependent signalling pathway as VEA and, if so, whether CSN and VEA interact post-transcriptionally remains to be elucidated.

Nothing is known about other incoming signals yet. During the course of growth and development, the level of $\operatorname{csn} D$ transcripts remained fairly constant as shown in Chapter 4. Nevertheless, it cannot be excluded that transcription of csn genes is increased within a narrow time window during acquisition of competence or in the course of development. It is noteworthy that the 5'-regulatory regions of both $A$. nidulans csn genes include putative binding sites for several transcription factors of $A$. nidulans (see Tab. 1.2 for references). The $\operatorname{csn} D$ and $\operatorname{csn} E$ promoters both contain two putative response elements for the AREA transcription factor that controls nitrogen metabolism (5'-A/T/C GATA G/A-3'). Several additional 5'-GATA-3' stretches might contribute to regulation by other GATA-like transcription factors like AREB, SREA and NSDD. The csnE regulatory region contains two putative CPCA response elements and one possible STUA binding site (5'ATT CGCG ATT N A/C-3'), whereas the $\operatorname{csn} D$ promoter includes only imperfect CPCA and STUA consensus sequences. Additionally, a putative CREA response element $\left(5^{\prime}-\mathrm{C} / \mathrm{G} \mathrm{C} / \mathrm{T} \mathrm{GG} \mathrm{G/A} \mathrm{G-}\right.$ $\left.3^{\prime}\right)$ is found in the csnE promoter as well as putative ANCF (5'-CCAAT-3') and PACC (5'-GCCA G/A G-3') binding sites in the $\operatorname{csn} D$ promoter. It seems promising to 
monitor expression of the CSN subunits in respect to the regulatory pathways affected by these diverse transcription factors. Overall, such a broad range of putative transcriptional control might indicate that expression of the COP9 signalosome is probably controlled by a variety of regulatory pathways, including signal transductions that finally control metabolism and development.

One important part of signal transduction in eukaryotes essential to respond to external stimuli are heterotrimeric $G$ proteins. A link of the COP9 signalosome with G-protein mediated signal transduction is conceivable. The human CSN subunit 1 (former called Sgn1, Gps1) suppresses a constitutively active G $\beta \gamma$ heterodimer in the corresponding yeast $G \alpha$-deletion strain in respect to the pheromone response pathway (Spain et al. 1996). With FADA, SFAD and FLBA (see Tab. 1.2 for references), components of a heterotrimeric $G$ protein signalling pathway have been identified in $A$. nidulans and cross-connection of this regulatory path to the CSN-dependent network might be questioned in future studies.

The COP9 signalosome functions as modulator of E3 ubiquitin ligases by the associated enzyme activities of a deneddylase and a protein kinase (see 1.2.2.2). An analogous function of the multiprotein complex may be predicted for the A. nidulans COP9 signalosome - but remains to be proven by appropriate phosphorylation and neddylation assays. A first insight into CSN activities in filamentous fungi might be gained from studies concerning functional complementation of $A$. nidulans csn deletion strains with the corresponding heterologous counterparts. Strikingly, deletion of csn genes is lethal in the plant $A$. thaliana and the fruit fly $D$. melangolaster, but not in the filamentous fungus A. nidulans. Functional complementation of the A. nidulans $\operatorname{csn} D$ deletion strain with the cDNA of the A. thaliana CSN4 subunit (Serino et al. 1999) will at least answer whether the function of this particular subunit is conserved between higher and lower eukaryotes. To date, six subunits of the S. pombe COP9 signalosome have been identified, whereas the yeast genome contains only one open reading frame with significant identities to a CSN subunit (see 1.2.2.4). In the A. nidulans genome sequence of the Cereon database (http://microbial.cereon.com), which is not yet complete, putative homologues to other CSN subunits seem present but only vague similarities are found for subunits seven and eight. This raises the question whether the multiprotein complex might be of different size in lower and higher eukaryotes. In this work, the $\operatorname{csn} D$ gene was cloned in a yeast expression vector (pME2359) and overexpressed in yeast, which was at least not lethal. Isolation of the A. nidulans CSN subunit 5 (CSNE) and the corresponding deletion strain (Chapter 4) provide an important tool to study the significance of the putative CSN5 homologue of yeast. Further studies might show whether the proposed yeast CSN5 functionally complements the mutant phenotypes of the A. nidulans csnE deletion strain.

Since malfunction of the COP9 signalosome results in multifaceted mutant phenotypes in $A$. nidulans, the CSN-dependent network seems to be involved in the regulation of several downstream circuits. Two hybrid interaction screens or pulldown assays might identify putative target proteins. If a similar function of the CSN in A. nidulans compared to the higher eukaryotic systems is assumed, a potential 

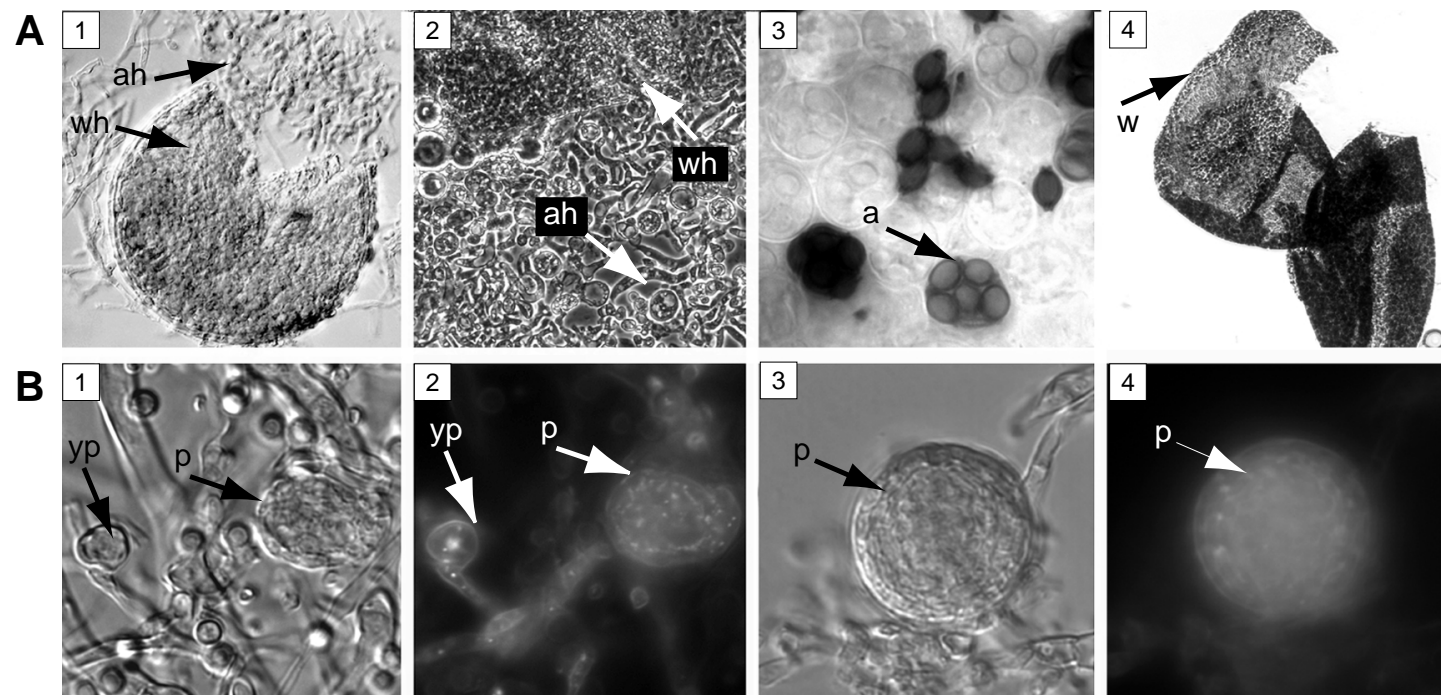

Fig. 5.2: Maturation sexual tissue types in $\boldsymbol{A}$. nidulans. (A) Sexual tissue types in an A. nidulans wild-type strain. A very young micro-cleitothecium shows two separate specialised types of hyphae: wall hyphae (wh) and ascogenous hyphae (ah) (1). Both hyphae further differentiate, the wall hyphae get flattened and glued and the the ascogenous hyphae show rounded cells (2). In the mature cleistothecium, the ascogenous hyphae have fully differentiated into asci (a) containing eight ascospores each and the wall hyphae into a hard wall (w) (4). (B) Autofluoresce might be a suiteable tool to recognise early sexual developmental structures in $A$. nidulans. In a csn deletion strain, several putative stages of young (yp) and older primordia (p) were recogniseable upon light $(1,3)$ and fluorescence $(2,4)$ microscopy.

target protein of the COP9 signalosome might have already been cloned from A. nidulans recently, the proposed culA-encoded cullin (Eckert 2000). However, up to now a connection between this cullin and the CSN is only hinted at the shared acleistothecial phenotype and the knowledge of a CSN-associated cullindeneddylation function in other organisms (see 1.2.2.2).

Strikingly, the CPCA homologue of mammalian cells, c-Jun, is among the CSN target proteins in higher eukaryotes (Claret et al. 1996). A direct interaction was shown for this oncogene with subunit 5 (CSN5) that was thus formerly termed Jun activation domain binding protein (JAB1). The c-Jun protein is phosphorylated by the CSN which prevents its ubiquitinylation and subsequent degradation (Naumann et al. 1999; Chamovitz and Segal 2001). Thus, it seems promising for future studies to question a link between the cross-pathway control and the COP9 signalosome in A. nidulans. A first approach should examine the response of the csn deletion strains to amino acid starvation conditions, accompanied by monitoring of CPCA protein stability upon starvation and non-starvation conditions in these strains.

\subsection{Metabolism and development of $\boldsymbol{A}$. nidulans}

Little is known about the molecular processes that drive secondary metabolism and sexual development in A. nidulans. Thus, the multifaceted impact of crosspathway control and the COP9 signalosome-dependent network on physiological as well as morphological traits constitutes a promising starting point for future 
analyses. Especially the developmental block at distinct intermediate stages during cleistothecia formation and a simultaneous dis-regulation of secondary metabolite production are of interest and may contribute to new insights about crossconnections between secondary metabolites and sexual development in A. nidulans.

\subsubsection{Morphogenesis of sexual fruit bodies}

An A. nidulans cleistothecium is an organised multi-cellular tissue. Nothing is known about the underlying morphogenetic events that lead to maturation of cleistothecial primordia into the characteristic shape of the sexual fruit body. The formation of cleistothecia includes two major maturation processes: from specialised sterile hyphae to a wall and from specialised fertile hyphae to ascospores (Fig. 5.2A). The first conception of the sexual reproductive structure is the cleistothecial primordium, in which at least on the visual level no separation between future wall and ascospores can be discriminated. Future microscopic analyses with isolated primordial structures should further examine this observation. Micro-cleistothecia represent an intermediate developmental stage in which the initial determination processes that control cell fate must have been successfully passed, since in isolated micro-cleistothecia a soft and pale coloured wall encloses ascogenous tissue. Acleistothecial A. nidulans strains have been described, but the defects in sexual development are restricted to either the absence of any type of sexual tissue or to production of solely Hülle cells (see 1.4.4.2). This work demonstrates that malfunction of the COP9 signalosome or activation of the cross-pathway control by impaired amino acid biosynthesis lead to a developmental arrest at the sexual stages of primordia or micro-cleistothecia, respectively. These regulatory networks are thus particularly suited to study morphogenesis of the sexual reproductive structures of $A$. nidulans.

Recently, CPCA of A. nidulans was characterised in more detail which revealed that, in contrast to the developmental arrest of a $c p c A$-overproduction strain, deletion of $c P c A$ results in no obvious developmental mutant phenotypes (Hoffmann et al. 2001). Additionally, preliminary experiments show that intermediate levels of CPCA might be the cause of an impressive phenomenon: eventual differentiation of mature ascospores that are not surrounded by a cleistothecial wall (Hoffmann 2000). Vice versa, A. nidulans mutant strains that produce empty cleistothecia consisting of a mature wall but lacking ascospores have been described (Swart et al. 2001). Thus, the maturation processes of wall and ascospores can probably proceed independently. Whether the phenotype of "open cleistothecia" is indeed due to the reduced level of CPCA remains to be proven. It seems of major concern for future studies to exactly analyse why this phenotype is instable and whether such open cleistothecia produce no wall at all or whether a first wall conception is build, like in micro-cleistothecia, that is not enlarged and modified during further differentiation and thus bursts.

If genesis of wall and ascospores are unlinked processes, it must be questioned whether the circular structure of the cleistothecial primordium 
represents a yet fully potent tissue or whether its cells fate is already determined. The csn deletion strains, arrested at the developmental stage of primordia, are thus useful for experimental setups that address questions of developmental biology. During the course of this work, autofluorescense has been helpful to identify early sexual stages in A. nidulans, particularly in the csn deletion strains (Fig. 5.2B). Generally, Aspergillus species produce autofluorescing substances (Mann 1983), but it was not yet examined whether quantity and quality of luminescence under the fluorescence microscope is identical in wild-type and csn mutant strains.

The results of this study show that the cross-pathway control negatively controls maturation processes whereas the COP9 signalosome is essential for either the onset or the progression of maturation. It was discussed above that a link between cross-pathway control and COP9 signalosome is conceivable. If a conserved function of the CSN in A. nidulans is proposed and an analogy to the mammalian system is taken into account, a stabilisation effect of COP9 signalosome towards CPCA should be examined (1.2.2.3.). An interplay of both regulatory networks would be of special interest regarding the control of sexual development. During the course of this work, an $A$. nidulans $\operatorname{csnD/cpcA}$ double deletion strain (AGB204) was constructed by crossing experiments (AGB192 $x$ AGB52), that completely resembled the $\triangle \operatorname{csn} D$ mutant phenotype. It should be questioned whether an $A$. nidulans strain deleted for a CSN subunit but with overproduction of CPCA still blocks sexual development at the level of primordia like a $\operatorname{csn} D$ deletion strain or proceeds until formation of micro-cleistothecia like a CPCA overproduction strain. Additionally, it would be interesting to question whether a modulated CSN activity in any case leads to aberrant cleistothecia lacking either solely the wall or the ascospores. However, a simple overexpression of single COP9 signalosome subunits is not promising and thus artificial modulation of overall CSN activity seems a challenge per se.

\subsubsection{Cross-connections of secondary metabolism and sexual development}

It is striking that changes in single enzyme activities within two different regulatory networks, the cross-pathway control and the COP9 signalosome-dependent system, impact sexual development as well as production of secondary metabolites in $A$. nidulans. Taken into account that in filamentous prokaryotes like Streptomyces coelicolor a biochemical transition from primary to secondary metabolism generally coincides with the onset of morphological differentiation (Vohradsky et al. 1997; Onaka et al. 1998), it might be worthwhile to question the molecular relationship between metabolism and development in filamentous fungi. Such cross-connections may rely on production of specific signal molecules like the FluG, PsiC and auxin (see 1.3.2.2), or on a co-regulation of metabolic and developmental pathways by a shared regulator. A well-studied example for a correlation between development and secondary metabolite production in A. nidulans is the proposed common regulation of the onset of asexual sporulation and biosynthesis of sterigmatocystin (see 1.3.2.1) (Adams and Yu 1998; Guzmande-Pena et al. 1998). It is hypothesised that sterigmatocystin and conidiospore 
production share a regulatory signal transduction cascade including FLBA as a mediator of G-protein signalling ( $Y u$ et al. 1996), the G-protein subunits FADA and SFAD (Hicks et al. 1997; Rosen et al. 1999; Tag et al. 2000) and the cAMPdependent protein kinase PKAA (Shimizu and Keller 2001). Both, sporulation and mycotoxin production, require inactivation of the FLBA/FADA-dependent signalling pathway (Hicks et al. 1997). In A. nidulans strains with an activated cross-pathway control, the sexual cycle is arrested (Chapter 2) and penicillin production is neglected (Chapter 3). This raises the important question whether these physiological and morphological phenotypes correlate, similar as described for sterigmatocystin biosynthesis and sporulation. It might be revealing to determine the penicillin titre in other acleistothecial or hypercleistothecial $A$. nidulans strains.

The developmental arrest in $c p c A$-overproduction strains (Chapter 2) might be due to either a specific effect of the CPCA transcriptional activator on a developmental regulator or to a general shortage of primary and secondary metabolites caused by activation of the cross-pathway control. It might be worthwhile trying to release the developmental block in amino acid starved mycelia by known signal molecules. A promising candidate might be auxin: recently, this tryptophan-related secondary metabolite was proven to promote development in A. nidulans. External supply of either high amounts of tryptophan or low amounts of auxin restored the developmental block of tryptophan-auxotrophic strains (Eckert et al. 1999; Eckert et al. 2000). It should now be tested whether auxin also enables maturation of cleistothecia in other amino acid auxotrophic strains, like the his $B$ deletion strain supplemented with low histidine concentrations, or in a $c p c A$ overproduction strain.

A. nidulans csn deletion strains not only arrest sexual development, but also produce an aberrant red pigment in some hyphae which indicates that secondary metabolism is affected. It is questionable whether de-repressed pigment synthesis in the $\operatorname{csn} D$ deletion strains somehow contributes to the block in sexual development. For the tyrosine-related pigment melanin, a correlation between defective hyphal melanin synthesis and defective sexual reproduction was described (Pirt and Rowley 1969; Polacheck and Rosenberger 1977). It might be revealing to delete the csn genes in A. nidulans strains with defects in its ascospore pigment production. Strains mutated in the $c / A / c / B$ or $b / A$ loci produce colourless or blue cleistothecia, respectively (Apirion, 1963). It is promising to examine whether malfunction of the COP9 signalosome in these strains still results in production of a red pigment in hyphae. Possibly, production of the red pigment is not the only pathway of secondary metabolism that is regulated by the COP9 signalosome-dependent network. Preliminary HPLC analysis of mycelia from the $\operatorname{csn} D$ deletion strain revealed that the chromatogram of mutant and wildtype strain differ, not in the quantity but in the constituent amount of the metabolites (Dr. H.B. Bode, personal communication). Similarly, for the pre-competence aco mutant strains impaired development as well as overproduction of phenolic substances were described (Butnick et al. 1984). Whether these changed metabolic profiles include signal molecules essential for development remains to be elucidated. It might be revealing to supply known developmental signal 
molecules, like the PsiC factor or auxin, to surface-grown csn deletion strains as a try to restore formation of a rigid wall or ascospores - or even a complete cleistothecia.

system of primary metabolism in yeast, is involved in the regulation of histidine biosynthesis as well as penicillin production and sexual development of the filamentous fungus Aspergillus nidulans. The COP9 signalosome-dependent network, known to control development of higher eukaryotes, contributes to regulation of light-dependent signalling, sexual development and pigment production in $A$. nidulans. These simultaneous effects on metabolism and development by both regulatory networks suggest several cross-connections between physiology and morphology. This includes several topics of more general interest, like determination of cell fate during development and the question about the biological role of secondary metabolites. The basic processes of the coordinated control of integration of environmental stimuli, physiology and morphology can be studied in the eukaryotic mikroorganism $A$. nidulans, and it will be interesting to transfer the knowledge learned from this model organism to higher eukaryotic systems.

\subsection{References}

Adams, T.H. and J.H. Yu. 1998. Coordinate control of secondary metabolite production and asexual sporulation in Aspergillus nidulans. Curr Opin Microbiol 1: 674-677.

Apirion, D. 1963. Formal and physiological genetics of ascospore colour in Aspergillus nidulans b. Genet Res 4: $276-283$.

Butnick, N.Z., L.N. Yager, M.B. Kurtz, and S.P. Champe. 1984. Genetic analysis of mutants of Aspergillus nidulans blocked at an early stage of sporulation. J Bacteriol 160: 541-545.

Chamovitz, D.A. and D. Segal. 2001. JAB1/CSN5 and the COP9 signalosome. A complex situation. EMBO Rep 2: 96-101.

Claret, F.X., M. Hibi, S. Dhut, T. Toda, and M. Karin. 1996. A new group of conserved coactivators that increase the specificity of AP-1 transcription factors. Nature 383: 453-457.

Eckert, S.E. 2000. Tryptophan biosynthesis and sexual development of Aspergillus nidulans. In Dissertation, pp. 92-103, Friedrich-Alexander Universität Erlangen-Nürnberg.

Eckert, S.E., B. Hoffmann, C. Wanke, and G.H. Braus. 1999. Sexual development of Aspergillus nidulans in tryptophan auxotrophic strains. Arch Microbiol 172: 157-166.

Eckert, S.E., E. Kübler, B. Hoffmann, and G.H. Braus. 2000. The tryptophan synthase-encoding $\operatorname{trp} B$ gene of Aspergillus nidulans is regulated by the cross-pathway control system. Mol Gen Genet 263: 867-876.

Goc, A. and P. Weglenski. 1988. Regulatory region of the Aspergillus nidulans argB gene. Curr Genet 14: 425-429.

Guzman-de-Pena, D., J. Aguirre, and J. Ruiz-Herrera. 1998. Correlation between the regulation of sterigmatocystin biosynthesis and asexual and sexual sporulation in Emericella nidulans. Antonie van Leeuwenhoek 73: 199-205.

Hicks, J.K., J.H. Yu, N.P. Keller, and T.H. Adams. 1997. Aspergillus sporulation and mycotoxin production both require inactivation of the $\mathrm{FadA} G \alpha$ protein-dependent signaling pathway. EMBO J 16: 49164923.

Hoffmann, B. 2000. Wechselwirkung zwischen der Regulation der Aminosäurebiosynthese und der Fruchtkörperbildung in dem filamentösen Pilz Aspergillus nidulans. In Dissertation, pp. 132-137, Friedrich-Alexander Universität Erlangen-Nürnberg.

Hoffmann, B., O. Valerius, M. Andermann, and G.H. Braus. 2001. Transcriptional autoregulation and inhibition of mRNA translation of amino acid regulator gene $c p c A$ of filamentous fungus Aspergillus nidulans. Mol Biol Cell 12: 2846-2857. 
Mann, J.L. 1983. Autofluorescence of fungi: an aid to detection in tissue sections. Am J Clin Pathol 79: 587590.

Mavrothalassitis, G., G. Beal, and T.S. Papas. 1990. Defining target sequences of DNA-binding proteins by random selection and PCR: determination of the GCN4 binding sequence repertoire. DNA Cell Biol 9: 783-788.

Naumann, M., D. Bech-Otschir, X. Huang, K. Ferrell, and W. Dubiel. 1999. COP9 signalosome-directed C-Jun activation/stabilization is independent of JNK. J Biol Chem 274: 35297-35300.

Oliphant, A.R., C.J. Brandl, and K. Struhl. 1989. Defining the sequence specificity of DNA-binding proteins by selecting binding sites from random-sequence oligonucleotides: analysis of yeast GCN4 protein. Mol Cell Biol 9: 2944-2949.

Onaka, H., T. Nakagawa, and S. Horinouchi. 1998. Involvement of two A-factor receptor homologues in Streptomyces coelicolor A3(2) in the regulation of secondary metabolism and morphogenesis. Mol Microbiol 28: 743-753.

Pirt, S.J. and B.I. Rowley. 1969. Melanin production in Aspergillus nidulans. Biochem J 114: 9-10.

Polacheck, I. and R.F. Rosenberger. 1977. Aspergillus nidulans mutant lacking $\alpha-(1,3)$-glucan, melanin, and cleistothecia. J Bacteriol 132: 650-656.

Rosen, S., J.H. Yu, and T.H. Adams. 1999. The Aspergillus nidulans sfaD gene encodes a $\mathrm{G}$ protein $\beta$ subunit that is required for normal growth and repression of sporulation. EMBO J 18: 5592-5600.

Sattlegger, E., A.G. Hinnebusch, and I.B. Barthelmess. 1998. cpc-3, the Neurospora crassa homologue of yeast GCN2, encodes a polypeptide with juxtaposed elF2 $\alpha$ kinase and histidyl-tRNA synthetaserelated domains required for general amino acid control. J Biol Chem 273: 20404-20416.

Serino, G., T. Tsuge, S. Kwok, M. Matsui, N. Wei and X-W. Deng. 1999. Arabidopsis cop8, and fus4 mutations define the same gene that encodes subunit 4 of the COP9 signalosome. The Plant Cell 11: 1967-1979.

Shimizu, K. and N.P. Keller. 2001. Genetic involvement of a cAMP-dependent protein kinase in a G protein signaling pathway regulating morphological and chemical transitions in Aspergillus nidulans. Genetics 157: 591-600.

Spain, B.H., K.S. Bowdish, A.R. Pacal, S.F. Staub, D. Koo, C.Y. Chang, W. Xie, and J. Colicelli. 1996. Two human cDNAs, including a homolog of Arabidopsis FUS6 (COP11), suppress G-protein- and mitogenactivated protein kinase-mediated signal transduction in yeast and mammalian cells. Mol Cell Biol 16: 6698-6706.

Strittmatter, A.W., S. Irniger, and G.H. Braus. 2001. Induction of jlbA mRNA synthesis for a putative bZIP protein of Aspergillus nidulans by amino acid starvation. Curr Genet 39: 327-334.

Swart, K., D. van Heemst, M. Slakhorst, F. Debets, and C. Heyting. 2001. Isolation and characterization of sexual sporulation mutants of Aspergillus nidulans. Fungal Genet Biol 33: 25-35.

Tag, A., J. Hicks, G. Garifullina, C. Ake, Jr., T.D. Phillips, M. Beremand, and N. Keller. 2000. G-protein signalling mediates differential production of toxic secondary metabolites. Mol Microbiol 38: 658-665.

Valerius, O., O. Draht, E. Kübler, K. Adler, B. Hoffmann, and G.H. Braus. 2001. Regulation of hisHF transcription of Aspergillus nidulans by adenine and amino acid limitation. Fungal Genet Biol 32: 2131.

Vohradsky, J., X.M. Li, and C.J. Thompson. 1997. Identification of procaryotic developmental stages by statistical analyses of two-dimensional gel patterns. Electrophoresis 18: 1418-1428.

Wek, S.A., S. Zhu, and R.C. Wek. 1995. The histidyl-tRNA synthetase-related sequence in the elF-2 $\alpha$ protein kinase GCN2 interacts with tRNA and is required for activation in response to starvation for different amino acids. Mol Cell Biol 15: 4497-506.

Yu, J.H., J. Wieser, and T.H. Adams. 1996. The Aspergillus FlbA RGS domain protein antagonizes G protein signaling to block proliferation and allow development. EMBO J 15: 5184-5190. 


\section{Acknowledgements}

In the first place I want to thank Gerhard Braus for supervision and support of my work. His immense optimism and inspiring ideas in scientific discussions were invaluable for motivation. I am particularly thankful that he enabled me to present parts of my work on international conferences.

I thank Prof. Dr. G. Gottschalk for accepting to co-examine this thesis.

I appreciate the works of Iris Nörenberg, Helge Woldt and Helge Bode who contributed to this work in their diploma theses. With special thanks to Helge Bode for interesting discussions about biosynthesis of antibiotics and production of pigments in microorganisms! For additional helpful discussions and strains for the 'penicillin project' I want to thank Prof. Dr. Axel Brakhage.

Thanks to my present and former colleagues for the pleasant working atmosphere in the lab. I especially want to thank the Aspergillus-fraction for mutual help and motivation. Sabine Eckert initially excited my interest for filamentous fungi with her enthusiasm when telling about Aspergillus. Her work, especially the REMI mutagenesis, was an important basis for my studies. I owe special thank to Sven Krappmann, who convinced me to hold on after setbacks during my work and always took time for helpful discussions. I thank Oliver Draht for sharing his 'australian'-Aspergillus-knowledge, a great time at the Asilomar conference and countless coffee breaks. Also, special thanks to Verena Große for help in sequence analysis as well as crossing and transformation experiments with Aspergillus strains. Another 'thank you' to Kerstin Helmstedt, whom I admire for her competence, correctness and organisation.

Thanks to all present and former members of the group: Meike Andermann, Melanie Bolte, Patrick Dieckhoff, Katrin Düvel, Olaf Grungmann, Gabi Heinrich, Stefan Irniger, Malte Kleinschmidt, Tim Köhler, Maria Meyer, Hans-Ueli Mösch, Heidi Northemann, Andrea Pfeil, Ralph Pries, Axel Strittmatter, Naimeh Taheri, Oliver Valerius and Claudia Wagner - for discussions, ideas and several nonscientific events.

I additionally want to thank all present and former members of the institute that supported me during the time-course of my work, especially Caroline ToecheMittler, Roman Grabbe, Kai Klopprogge and Olivia Gohl. I also thank my 'ex-255er lab' for staying in contact with annual meetings.

Last but not least, I want to thank my family and friends for giving me a lot of general support all the time!

The Deutsche Forschungsgemeinschaft, the Fond der chemischen Industrie and the Volkswagen-Stiftung have granted financial support for this work. 



\title{
Curriculum vitae
}

\author{
Silke Busch \\ Born on March the $12^{\text {th }}, 1971$ \\ in Vechta, Germany.
}

\section{Education}

1978 - 1984 Primary education in Diepholz (GER)

1984 - 1991 Secondary education at the Graf-Friedrich-Schule in Diepholz (GER)

\section{Scientific Background}

1991 - 1993 Study of biology at the Universität Osnabrück (GER)

1993 - 1994 Study of biology at the University of Sussex, Brighton (UK)

1994 - 1998 Study of biology at the Georg-August Universität Göttingen (GER). Graduation in biology with the diploma thesis "Untersuchung des natürlichen DNA-Transformationssystems in Acinetobacter sp. BD413: Identifizierung und Charakterisierung der Kompetenzgene comE und comF" in the Institut für Mikrobiologie und Genetik, Abteilung für Allgemeine Mikrobiologie of Prof. Dr. G. Gottschalk

1998-2002 Scientific assistant at the Georg-August Universität Göttingen (GER), in the Institut für Mikrobiologie und Genetik, Abteilung für Molekulare Mikrobiologie und Genetik of Prof. Dr. G.H. Braus 
